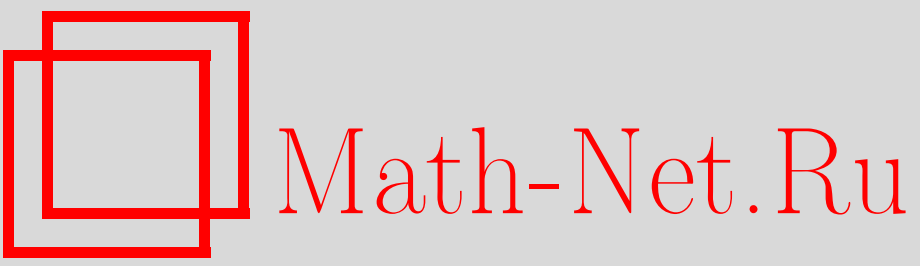

В. М. Бухштабер, Т. Е. Панов, Действия торов, комбинаторная топология и гомологическая алгебра, УМH, 2000, том 55, выпуск 5, 3-106

DOI: https://doi.org/10.4213/rm320

Использование Общероссийского математического портала Math-Net.Ru подразумевает, что вы прочитали и согласны с пользовательским соглашением

http://www.mathnet.ru/rus/agreement

Параметры загрузки:

IP : 54.80 .73 .141

26 апреля 2023 г., 17:59:17 


\title{
ДЕЙСТВИЯ ТОРОВ, КОМБИНАТОРНАЯ ТОПОЛОГИЯ И ГОМОЛОГИЧЕСКАЯ АЛГЕБРА
}

\author{
В. М. БУХШТАБЕР, Т. Е. ПАНОВ
}

Дан обзор результатов и открытых проблем, связанных с такими фундаментальными комбинаторными объектами как многогранники, симплициальные комплексы, кубические комплексы и конфигурации подпространств. Особое внимание уделено симплициальным и кубическим разбиениям многообразий и, в первую очередь, сфер. Изложены важные конструкции, позволяющие исследовать эти объекты средствами коммутативной и гомологической алгебры. В основе предлагаемого подхода ко всему кругу рассматриваемых проблем лежит развиваемая авторами теория момент-угол комплексов. Ключевым в ней является построение по каждому симплициальному комплексу $K$ с $m$ вершинами пространства $\mathscr{Z}_{K}$ с действием $m$-мерного тора, обладающего специальным биградуированным клеточным разбиением. В рамках этой теории широко известные неособые торические многообразия появляются как фактор-пространства момент-угол комплексов для симплициальных сфер по максимальному свободному действию тора. Показано, что инварианты симплициальных комплексов и связанных с ними комбинаторно-геометрических объектов выражаются в терминах биградуированных колец когомологий соответствующих момент-угол комплексов. Описаны решения ряда известных топологических задач, полученные на основе новых взаимосвязей комбинаторики, геометрии и топологии.

Библиограбия: 89 названий.

\section{СОДЕРЖАНИЕ}

Глава 1. Необходимые алгебраические, геометрические и комбинаторные понятия

$\S 1.1$. Многогранники

§1.2. Симплициалныте комплексы: топология и комбинаторика .............

§1.3. Симплициальные комплексы: коммутативная алгебра ...............

$\S$ 1.4. Гомологические свойства колец граней (колец Стенли-Райснера) ....

$\S 1.5$. Кубические комплексы и кубические отображения ................

$\S 2.1$. Алгебраические торические многообразия ...................... . .

$\S 2.2$. Квазиторические многообразия

§ 2.3. Стабильно комплексные структуры и квазиторические представители в классах кобордизмов

§ 2.4. Комбинаторные формулы для родов Хирцебруха квазиторических многообразий

$\S 2.5$. Задача классификации квазиторических многообразий над заданным простым многогранником 
Глава 3. Момент-угол комплексы

$\S$ 3.1. Момент-угол многообразия $\mathscr{Z}_{P}$, определяемые простыми многогранни-

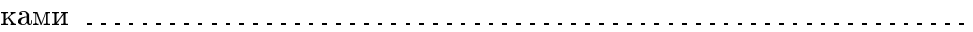

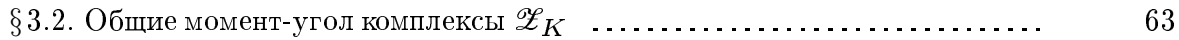

§3.3. Клеточные структуры на момент-угол комплексах ................. 64

§3.4. Конструкция Бореля и пространство Стенли-Райснера .......... 66

$\S 3.5$. Обобщения, аналоги и дополнительные комментарии ............... 69

Глава 4. Когомологии момент-угол комплексов и комбинаторика сим-

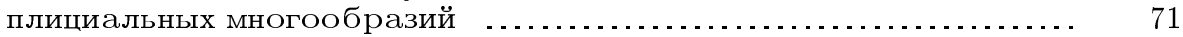

§4.1. Спектральная последовательность Эйленберга-Мура ............. 71

$\S 4.2$. Когомологии момент-угол комплекса $\mathscr{Z}_{K}$ : случай общего $K \ldots \ldots . . .$.

$\S 4.3$. Когомологии момент-угол комплекса $\mathscr{Z}_{K}$ : случай сферического $K \quad$.. $\quad 81$

$\S 4.4$. Фактор-пространства многообразия $\mathscr{Z}_{P}$ по свободным действиям тора 84

$\S 4.5$. Биградуированная двойственность Пуанкаре и аналоги соотношений Дена-Соммервилля для симплициальных многообразий .............

Глава 5. Конфигурации подпространств и кольца когомологий их дополнений

$\S 5.1$. Обзор результатов о когомологиях дополнений к общим конфигурациям

$\S 5.2$. Конфигурации координатных подпространств и когомологии комплекca $\mathscr{Z}_{K}$

§5.3. Конфигурации диагональных подпространств и когомологии пространства петель $\Omega \mathscr{Z}_{K}$

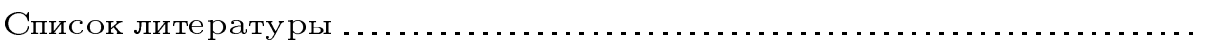

Обзор посвящен результатам и открытым проблемам в обширной области исследований, которая сформировалась за последние 20 лет на стьке комбинаторики многогранников, комбинаторной и алгебраической топологии, гомологической алгебры, теории действий групп на топологических пространствах, алгебраической геометрии торических многообразий и симплектической геометрии. Основная цель обзора показать, что предлагаемая авторами теория момент-угол комплексов позволяет существенно развить взаимосвязи между всеми перечисленными вьше областями математики и получить на основе этого решение ряда известных задач. Каждый параграф обзора относится, как правило, к отдельному направлению исследований и содержит соответствуюшее введение. В качестве путеводителя по обзору приведем схематическое изложение его содержания.

В главе 1 собраны все необходимые комбинаторные, геометрические и топологические сведения о многогранниках, симплициальных и кубических комплексах и разбиениях многообразий. Описаны как классические, так и оригинальные конструкции, позволяющие исследовать комбинаторные объекты методами коммутативной и гомологической алгебры.

В $\S 1$ главы 2 дан обзор используемых в дальнейшем конструкций и результатов об алгебраических торических многообразиях и связанных с ними комбинаторных объектах. Параграф 2.2 посвящен топологическим аналогам торических многообразий квазиторическим многообразиям, основы теории которых были заложены Дэвисом и

Работа выполнена при поддержке Российского фонда фундаментальных исследований (грант № 99-01-00090). 
Янушкевичем. Мы приводим важнейшие конструкции и результаты о квазиторических многообразиях. В $\S 2.3$ изложено полученное Бухштабером и Рэем решение аналога для квазиторических многообразий проблемы Хирцебруха о связных представителях в классе кобордизмов каждого стабильно комплексного многообразия. В 22.4 изложены полученные Пановым комбинаторные формулы для родов Хирцебруха квазиторических многообразий. В последнем параграфе главы 2 описаны результаты по классификации торических и квазиторических многообразий над заданньм простьм многогранником.

В главах 3 и 4 изложена развиваемая авторами теория момент-угол комплексов. Такой комплекс $\mathscr{Z}_{K}$ сопоставляется каждому симплициальному комплексу $K$ с $m$ вершинами (см. $\S 3.2$ ). На $\mathscr{Z}_{K}$ имеется каноническое действие тора $T^{m}$, пространством орбит которого является конус над $K$, и соответствуюшее биградуированное клеточное разбиение (см. §3.3). Ряд результатов, полученных при помощи этой теории, опирается на тот факт, что $\mathscr{Z}_{K}$ является многообразием в случае, когда $K$ - симплициальная сфера. Важные следствия вытекают также из того, что в более общем случае симплипиального многообразия $K$ особые точки комплекса $\mathscr{Z}_{K}$ образуют орбиту действия тора $T^{m}$, дополнение к окрестности которой представляет собой многообразие с краем. В главе 4 при помоши биградуированной клеточной структуры введено и вычислено биградуированное кольцо когомологий момент-угол комплекса $\mathscr{Z}_{K}$. Ответ записан в терминах известных конструкций из гомологической алгебры, используемых в решениях комбинаторных задач.

Глава 5 посвяшена приложениям теории момент-угол комплексов к известной задаче вычисления когомологий дополнений конфигураций подпространств. Основное внимание уделено конфигурациям координатных подпространств и конфигурациям диагональных подпространств ( $\S 5.2$ и 5.3 соответственно). Дан обзор результатов, полученных в этом направлении различными авторами. Приведено полученное нами решение задачи о кольце когомологий дополнения конфигурации координатных подпространств, основанное на редукции к вычислению кольца когомологий комплекса $\mathscr{Z}_{K}$. Описана редукция задачи вычисления когомологий диагональных подпространств к задаче вычисления когомологий пространства петель $\Omega \mathscr{Z}_{K}$.

Все изложение сопровождается примерами важнейших из рассматриваемых объектов и вычислениями их инвариантов, демонстрируюшими полученные общие результаты. Результаты, вошедшие в обзор, делятся на три части. Во-первых, это интерпретации классических результатов, которые иногда не сопровождаются ссылками. Во-вторых, результаты других авторов, которые мы сопровождаем соответствующей ссылкой. И в-третьих, результаты, которые либо получены нами недавно, либо являются развитием результатов, опубликованных в работах авторов [19]-[23], [69], [70] и в совместных работах [25], [26] с Н. Рэем.

Мы глубоко благодарны Л.А. Алания, В. А. Васильеву, Ф. Велкеру, Н.Э. Добринской, Н. П. Долбилину, И. Э. Изместьеву, О. Р. Мусину, С. П. Новикову, Э. Рису, Н. Рэю, Ю. Сивану, В.А. Смирнову, Н. Стрикланду, С.П. Тарасову, М. Фарберу, Г. Циглеру, М. А. Штанько, М. И. Штогрину и С. Юзвинскому за поддержку и вдохновение, полученные в ходе многочисленных обсуждений вопросов, которым посвящен данный обзор. Мы также благодарны участникам семинара "Топология и вычислительная геометрия", который проводится на механико-математическом факультете МГУ под руководством авторов и О.Р. Мусина. 


\section{Глава 1. Необходимые алгебраические, геометрические и комбинаторные понятия}

§1.1. Многогранники. Комбинаторные и геометрические аспекты теории выпуклых многогранников изложены в большом количестве учебных пособий, монографий и статей. Мы упомянем лишь книгу Циглера [89], где также можно найти дальнейшие ссылки. В этом параграфе мы описьваем основные понятия и конструкции, связанные с многогранниками, используемые в работе.

Имеется два различных подхода к определению выпуклого многогранника в $n$-мерном аффинном пространстве $\mathbb{R}^{n}$.

ОПРЕДЕЛЕНИЕ 1.1.1. Выпуклым многогранником назьвается выпуклая оболочка произвольного конечного множества точек в некотором пространстве $\mathbb{R}^{n}$.

ОПРЕДЕЛЕНИЕ 1.1.2. Выпуклым полиәдром Р назьвается пересечение конечного числа полупространств некоторого пространства $\mathbb{R}^{n}$ :

$$
P=\left\{\boldsymbol{x} \in \mathbb{R}^{n}:\left\langle\boldsymbol{l}_{i}, \boldsymbol{x}\right\rangle \geqslant-a_{i}, i=1, \ldots, m\right\},
$$

где $\boldsymbol{l}_{i} \in\left(\mathbb{R}^{n}\right)^{*}, i=1, \ldots, m,-$ некоторые линейные функции и $a_{i} \in \mathbb{R}$. Выпукльцм многогранником назьвается ограниченньй выпуклый полиэдр.

Тем не менее, эти два определения вьпуклого многогранника задают один и тот же геометрический объект, т.е. подмножество пространства $\mathbb{R}^{n}$ является вьпуклой оболочкой конечного набора точек тогда и только тогда, когда оно является пересечением конечного числа полупространств и ограничено. Этот факт доказывается во многих учебниках по теории многогранников и вьпуклой геометрии, см., например, [89; теорема 1.1].

Размерностью многогранника называется размерность его аффинной оболочки; в тех случаях, когда не оговаривается противное, мы рассматриваем только $n$-мерные многогранники $P^{n}$ в $n$-мерном пространстве $\mathbb{R}^{n}$. Опорной гиперплоскостью многогранника $P^{n}$ называется произвольная аффинная гиперплоскость $H$, которая пересекает $P^{n}$ и для которой многогранник содержится в одном из определяемых ею подпространств. Пересечения $P^{n} \cap H$ многогранника с опорными гиперплоскостями называются гранями многогранника. Сам многогранник $P^{n}$ также считается грањњю; все остальные грани называются собственныци. Границей $\partial P^{n}$ многогранника назьвается объединение всех его собственных граней. Каждая грань $n$-мерного многогранника $P^{n}$ является многогранником размерности $\leqslant n$. 0 -мерные грани назьваются вериинами, 1-мерные грани называются ребрами, а грани коразмерности один называются гипергранями. Грани многогранника $P^{n}$ всех размерностей образуют частично упорядоченное множество по отношению к включению, называемое решеткой граней многогранника $P^{n}$.

Два многогранника $P_{1} \in \mathbb{R}^{n_{1}}$ и $P_{2} \in \mathbb{R}^{n_{2}}$ одной размерности называются аффинно әквивалентными, если сушествует аффинное отображение $\mathbb{R}^{n_{1}} \rightarrow \mathbb{R}^{n_{2}}$, переводящее один многогранник в другой. Говорят, что два многогранника комбинаторно эквивалентнь, если сушествует биекция между их наборами граней, сохраняюшая отношение включения. Другими словами, два многогранника комбинаторно эквивалентны, если их решетки граней изоморфны как частично упорядоченные множества. 
ПримеР 1.1.3 (симплекс и куб). Симплекс $\Delta^{n}$ (n-симплекс) представляет собой выпуклую оболочку набора из $(n+1)$ точек в $\mathbb{R}^{n}$, не лежащих на одной аффинной гиперплоскости. Все грани $n$-симплекса являются симплексами размерности $\leqslant n$.

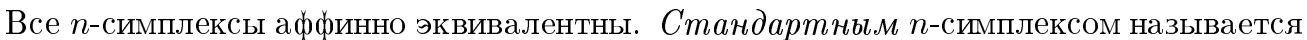
вьпуклая оболочка точек $(1,0, \ldots, 0),(0,1, \ldots, 0), \ldots,(0, \ldots, 0,1)$ и $(0, \ldots, 0)$ в $\mathbb{R}^{n}$. Стандартный $n$-симплекс задается $(n+1)$ неравенствами

$$
x_{i} \geqslant 0, i=1, \ldots, n, \quad \text { и }-x_{1}-\cdots-x_{n} \geqslant-1 .
$$

Правильный $n$-симплекс есть выпуклая оболочка $(n+1)$ точек $(1,0, \ldots, 0),(0,1$, $\ldots, 0), \ldots,(0, \ldots, 0,1)$ в в $\mathbb{R}^{n+1}$.

Стандартный q-куб есть вьпукльй многогранник $I^{q} \subset \mathbb{R}^{q}$, определяемьй как

$$
I^{q}=\left\{\left(y_{1}, \ldots, y_{q}\right) \in \mathbb{R}^{q}: 0 \leqslant y_{i} \leqslant 1, i=1, \ldots, q\right\} .
$$

В то же время стандартный $q$-куб представляет собой выпуклую оболочку $2^{q}$ точек в $\mathbb{R}^{q}$, имеюших лишь нулевые или единичные координаты.

Следуюшая конструкция позволяет задавать выпукльй $n$-мерный многогранник, имеюший $m$ гиперграней, как пересечение положительного конуса

$$
\mathbb{R}_{+}^{m}=\left\{\left(y_{1}, \ldots, y_{m}\right) \in \mathbb{R}^{m}: y_{i} \geqslant 0, i=1, \ldots, m\right\} \subset \mathbb{R}^{m}
$$

с некоторой $n$-мерной плоскостью.

Конструкция 1.1.4. Пусть $P \in \mathbb{R}^{n}$ - вьпуклый $n$-мерньй многогранник, заданный формулой (1) для некоторых $\boldsymbol{l}_{i} \in\left(\mathbb{R}^{n}\right)^{*}, a_{i} \in \mathbb{R}, i=1, \ldots, m$. Введем $(n \times m)$-матригу $L$, столбшы которой суть векторы $\boldsymbol{l}_{i}$, записанные в стандартном базисе пространства $\left(\mathbb{R}^{n}\right)^{*}$, т.е. $(L)_{j i}=\left(\boldsymbol{l}_{i}\right)_{j}$. Заметим, что ранг $L$ равен $n$. Введем также вектор-столбец $\boldsymbol{a}=\left(a_{1}, \ldots, a_{m}\right)^{t} \in \mathbb{R}^{m}$. Тогда мы можем переписать (1) как

$$
P=\left\{\boldsymbol{x} \in \mathbb{R}^{n}:\left(L^{t} \boldsymbol{x}+\boldsymbol{a}\right)_{i} \geqslant 0, i=1, \ldots, m\right\},
$$

где $L^{t}$ - транспонированная матрица и $\boldsymbol{x}=\left(x_{1}, \ldots, x_{n}\right)^{t}$ - вектор-столбец. Рассмотрим аффинное отображение

$$
A_{P}: \mathbb{R}^{n} \rightarrow \mathbb{R}^{m}, \quad A_{P}(\boldsymbol{x})=L^{t} \boldsymbol{x}+\boldsymbol{a} \in \mathbb{R}^{m} .
$$

Его образом является некоторая $n$-мерная плоскость в $\mathbb{R}^{m}$, и $(5)$ показывает, что $A_{P}(P)$ есть пересечение этой плоскости с положительньм конусом $\mathbb{R}_{+}^{m}$. Пусть теперь $W$ - матрица размера $m \times(m-n)$ и ранга $(m-n)$ такая, что $L W=0$. Тогда легко видеть, что

$$
A_{P}(P)=\left\{\boldsymbol{y} \in \mathbb{R}^{m}: W^{t} \boldsymbol{y}=W^{t} \boldsymbol{a}, y_{i} \geqslant 0, i=1, \ldots, m\right\} .
$$

Заметим, что многогранники $P$ и $A_{P}(P)$ аффинно эквивалентны. 
ПримеР 1.1.5. Рассмотрим стандартный $n$-симплекс $\Delta^{n} \subset \mathbb{R}^{n}$, определяемый неравенствами (2). Он имеет $m=n+1$ гиперграней и, в обозначениях (1), $\boldsymbol{l}_{1}=(1,0, \ldots, 0)^{t}, \ldots, \boldsymbol{l}_{n}=(0, \ldots, 0,1)^{t}, \boldsymbol{l}_{n+1}=(-1, \ldots,-1)^{t}, a_{1}=\cdots=a_{n}=0$, $a_{n+1}=1$. Мы можем положить $W=(1, \ldots, 1)^{t}$ в конструкции 1.1.4. Таким образом, $W^{t} \boldsymbol{y}=y_{1}+\cdots+y_{m}, W^{t} \boldsymbol{a}=1$, и мы имеем

$$
A_{\Delta^{n}}\left(\Delta^{n}\right)=\left\{\boldsymbol{y} \in \mathbb{R}^{n+1}: y_{1}+\cdots+y_{n+1}=1, y_{i} \geqslant 0, i=1, \ldots, n\right\} .
$$

Это есть правильньй $n$-симплекс в $\mathbb{R}^{n+1}$.

ЗАМЕЧАНИЕ. Выпуклые многогранники, вводимые определениями 1.1.1 и 1.1.2, представляют собой геометрические объекты. В то же время можно рассматривать комбинаторные многогранники, которые представляют собой классы комбинаторно эквивалентных вьпуклых многогранников. Фактически, комбинаторный многогранник есть решетка граней (геометрического) многогранника, рассматриваемая как частично упорядоченное множество. В данном обзоре мы имеем дело как с геометрическими, так и с комбинаторными многогранниками.

Два различных определения выпуклых многогранников приводят к двум различным понятиям многогранников общего положения.

Набор из $m>n$ точек пространства $\mathbb{R}^{n}$ находится в общем положении, если никакие $(n+1)$ из них не лежат на одной аффинной гиперплоскости. С точки зрения определения 1.1.1 выпукльй многогранник является многогранником обшего положения, если он представляет собой выпуклую оболочку набора точек в общем положении. Отсюда вытекает, что все собственные грани такого многогранника суть симплексы, т.е. каждая гипергрань имеет минимальное количество вершин (а именно $n$ ). Такие многогранники назьваются симплиииальнылми.

Набор из $m>n$ гиперплоскостей $\left\langle\boldsymbol{l}_{i}, \boldsymbol{x}\right\rangle=-a_{i}, \boldsymbol{l}_{i} \in\left(\mathbb{R}^{n}\right)^{*}, \boldsymbol{x} \in \mathbb{R}^{n}, a_{i} \in \mathbb{R}$, $i=1, \ldots, m$, находится в общем положсении, если никакая точка не принадлежит более чем $n$ гиперплоскостям. $\mathrm{C}$ точки зрения определения 1.1 .2 выпукльй многогранник $P^{n}$ является многогранником общего положения, если ограничивающие его гиперплоскости (см. (1)) находятся в обшем положении, т.е. в каждой вершине сходится в точности $n$ гиперграней. Такие многогранники называются $n$ pocmы.мu.

Для любого выпуклого многогранника $P \subset \mathbb{R}^{n}$ определим его полярное множество $P^{*} \subset\left(\mathbb{R}^{n}\right)^{*}$ как

$$
P^{*}=\left\{\boldsymbol{x}^{\prime} \in\left(\mathbb{R}^{n}\right)^{*}:\left\langle\boldsymbol{x}^{\prime}, \boldsymbol{x}\right\rangle \geqslant-1 \text { для всех } \boldsymbol{x} \in P\right\} .
$$

ЗАмЕчАниЕ. Наше определение полярного множества взято из алгебраической геометрии торических многообразий и отличается от классического определения, принятого в вьпуклой геометрии. Классическое определение получается при замене неравенства " $\geqslant-1$ ” выше на " $\leqslant 1$ ". Наше полярное множество переводится в классическое симметрией с центром в 0 .

Как известно из вьпуклой геометрии, при условии $0 \in P$ полярное множество $P^{*}$ является выпуклым многогранником в сопряженном пространстве $\left(\mathbb{R}^{n}\right)^{*}$ и при этом $\left(P^{*}\right)^{*}=P$. Многогранник $P^{*}$ назьвается полярным (или двойственным ) к $P$. Более того, имеется обращающее порядок взаимно однозначное соответствие между решетками граней многогранников $P$ и $P^{*}$. В частности, если $P$ является простым, то $P^{*}$ - симплициален, и наоборот. 
ПримеР 1.1.6. Любой многоугольник (2-многогранник) является одновременно простым и симплициальным. В размерностях $\geqslant 3$ единственным многогранником, который одновременно является простым и симплициальным, является симплекс. Куб является простым многогранником. Полярным многогранником симплекса является симплекс. Полярньй многогранник куба назьвается ортоэдром (или гипероктаәдром). Трехмерный ортоэдр представляет собой октаэдр.

В дальнейшем мы будем обозначать простые многогранники латинскими буквами $P, Q$ и т. д., в то время как симплициальные многогранники будут обозначаться такими же буквами со звездочкой: $P^{*}, Q^{*}$ и т. д.

Каждая грань простого многогранника является простьм многогранником. Произведение $P_{1} \times P_{2}$ двух простых многогранников $P_{1}$ и $P_{2}$ также является простым многогранником. Пусть $P_{1}^{*}$ и $P_{2}^{*}$ - соответствующие полярные симплициальные многогранники. Тогда $P_{1}^{*} \circ P_{2}^{*}:=\left(P_{1} \times P_{2}\right)^{*}$ снова является симплициальным многогранником. Операция о на симплициальных многогранниках может быть непосредственно описана следуюшим образом. Реализуем $P_{1}^{*}$ в $\mathbb{R}^{n_{1}}$ и $P_{2}^{*}$ в $\mathbb{R}^{n_{2}}$ таким образом, что $0 \in P_{1}^{*}$ и $0 \in P_{2}^{*}$. Тогда $P_{1}^{*} \circ P_{2}^{*} \subset \mathbb{R}^{n_{1}} \times \mathbb{R}^{n_{2}}$ есть выпуклая оболочка объединения $P_{1}^{*} \subset \mathbb{R}^{n_{1}} \times 0$ и $P_{2}^{*} \subset 0 \times \mathbb{R}^{n_{2}}$.

Конструкция 1.1.7 (связная сумма двух простых многогранников). Пусть нам даны два простых многогранника $P^{n}$ и $Q^{n}$ одинаковой размерности $n$ с выделенными вершинами $v$ и $w$ соответственно. Неформально, связная сумма $P^{n} \#_{v, w} Q^{n}$ многогранника $P^{n}$ в вершине $v$ с многогранником $Q^{n}$ в вершине $w$ получается следуюшим образом. Мы "срезаем" вершину $v$ с многогранника $P^{n}$ и вершину $w$ с $Q^{n}$; затем, применив соответствующее проективное преобразование, мы получаем возможность "правильно" приклеить оставшуюся часть многогранника $P^{n}$ к оставшейся части $Q^{n}$ вдоль вновь образовавшихся гиперграней. Результат обозначается $P^{n} \#_{v, w} Q^{n}$. Ниже мы приводим строгое определение, следуя $[26 ; \S 6]$, которое будет использоваться далее.

Вначале введем $n$-мерный полиэдр $\Gamma^{n}$, получаемый как произведение стандартного $(n-1)$-симплекса $\Delta^{n-1}$ в подпространстве $\left\{\boldsymbol{x}: x_{1}=0\right\} \subset \mathbb{R}^{n}$ с первой координатной осью. Гиперграни $G_{r}$ полиэдра $\Gamma^{n}$, таким образом, имеют вид $\mathbb{R} \times D_{r}$, где $D_{r}-$ грани симплекса $\Delta^{n-1}, 1 \leqslant r \leqslant n$. Полиэдр $\Gamma^{n}$ и все его гиперграни $G_{r}$ разделены на две части, положительную и отрицательную, в зависимости от знака координаты $x_{1}$.

Занумеруем гиперграни многогранника $P^{n}$, сходящиеся в вершине $v$, как $E_{1}, \ldots$, $E_{n}$, а гиперграни $Q^{n}$, сходящиеся в $w$, как $F_{1}, \ldots, F_{n}$. Обозначим дополнительные множества гиперграней через $\mathscr{C}_{v}$ и $\mathscr{C}_{w}$ соответственно; таким образом, гиперграни из $\mathscr{C}_{v}$ не содержат $v$, а гиперграни из $\mathscr{C}_{w}$ не содержат $w$.

Выберем теперь два проективных преобразования $\phi_{P}$ и $\phi_{Q}$ пространства $\mathbb{R}^{n}$, которые переводят вершины $v$ и $w$ в $x_{1}= \pm \infty$ соответственно. Мы также потребуем, чтобы преобразование $\phi_{P}$ отображало $P^{n}$ внутрь полиэдра $\Gamma^{n}$ и удовлетворяло двум условиям: во-первых, гиперплоскость, содержашая гипергрань $E_{r}$, переходит в гиперплоскость, содержашую $G_{r}$, для любого $r=1, \ldots, n$, и, во-вторых, образы гиперплоскостей, содержаших гиперграни из множества $\mathscr{C}_{v}$, пересекают полиэдр $\Gamma^{n}$ в его отрищательной части. Аналогично, преобразование $\phi_{Q}$ переводит гиперплоскость, содержашую гипергрань $F_{r}$, в гиперплоскость, содержашую гипергрань $G_{r}$, для любого $1 \leqslant r \leqslant n$, но образы гиперплоскостей, содержаших гиперграни из $\mathscr{C}_{w}$, 
пересекают полиэдр $\Gamma^{n}$ в его положительной части. Определим теперь связную суммy $P^{n} \#{ }_{v, w} Q^{n}$ многогранника $P^{n}$ в вершине $v$ с многогранником $Q^{n}$ в $w$ как простой многогранник, ограничиваемьй образами гиперплоскостей, соответствующих гиперграням из $\mathscr{C}_{v}, \mathscr{C}_{w}$, и гиперплоскостями, содержащими гиперграни $G_{r}, r=1, \ldots, n$. Связная сумма определена лишь с точностью до комбинаторной эквивалентности; более того, она, вообше говоря, зависит от выбора вершин $v, w$ и от нумерации гиперграней $E_{r}$ и $F_{r}$. Если этот выбор определяется контекстом или же если результат не зависит от этого выбора, то мы будем использовать сокращенное обозначение $P^{n} \# Q^{n}$

Близкая конструкция связной суммы $P \# Q^{*}$ простого многогранника $P$ и симплициального многогранника $Q^{*}$ описана в [89; пример 8.41].

Симплициальный многогранник $P^{*}$ называется $k$-смежсностнылм, если любые $k$ его вершин порождают некоторую грань. Аналогично, простой многогранник $P$ называется $k$-смежностным, если любые $k$ его гиперграней имеют непустое пересечение (которое в этом случае является гранью коразмерности $k$ ). Очевидно, любой симплициальный (и простой) многогранник является 1-смежностным. Можно показать (см. [18; следствие 14.5], а также пример 1.1 .15 ниже), что если $P^{*}$ является $k$-смежностным симплициальным $n$-многогранником и $k>\left[\frac{n}{2}\right]$, то $P^{*}$ есть $n$-симплекс. В частности, любой 2-смежностньй симплициальный 3-многогранник есть симплекс (тетраэдр). При этом, однако, сушествуют симплициальные $\left[\frac{n}{2}\right]$-смежностные $n$-многогранники с любым числом вершин. Такие многогранники называются смежсностными. В частности, существует симплищиальньй 4-многогранник, отличньй от симплекса, каждые две вершины которого соединены ребром.

ПримеР 1.1 .8 (смежностньй 4-многогранник). Пусть $P=\Delta^{2} \times \Delta^{2}$ - произведение двух треугольников. Тогда $P$ является простым многогранником и, как легко проверить, любые две гиперграни в $P$ пересекаются по обшей 2-грани. Таким образом, $P$ является 2-смежностным. Полярный многогранник $P^{*}$ является смежностным симплициальным 4-многогранником.

Предыдущий пример допускает следуюшее обобщение. Пусть $P_{1}$ и $P_{2}$ - соответственно $k_{1}$-смежностный и $k_{2}$-смежностный простые многогранники. Тогда произведение $P_{1} \times P_{2}$ является $\min \left(k_{1}, k_{2}\right)$-смежностным простьм многогранником. Отсюда следует, что многогранники $\left(\Delta^{n} \times \Delta^{n}\right)^{*}$ и $\left(\Delta^{n} \times \Delta^{n+1}\right)^{*}$ дают примеры смежностных симплициальных многогранников размерностей $2 n$ и $(2 n+1)$ соответственно. Ниже приводится другой пример смежностного многогранника, который может иметь произвольное число вершин.

ПРИМЕР 1.1.9 (циклические многогранники). Определим моментную кривую в $\mathbb{R}^{n}$ как

$$
x: \mathbb{R} \rightarrow \mathbb{R}^{n}, \quad t \mapsto \boldsymbol{x}(t)=\left(t, t^{2}, \ldots, t^{n}\right) \in \mathbb{R}^{n}
$$

Для каждого $m>n$ определим ичклический многогранник $C^{n}\left(t_{1}, \cdots, t_{m}\right)$ как выпуклую оболочку $m$ различных точек $\boldsymbol{x}\left(t_{i}\right), t_{1}<t_{2}<\cdots<t_{m}$, на моментной кривой. Как следует из формулы для определителя Вандермонда, никакие $(n+1)$ точек на моментной кривой не лежат на одной аффинной гиперплоскости. Таким образом, $C^{n}\left(t_{1}, \ldots, t_{m}\right)$ является симплициальным $n$-многогранником. Можно показать (см. 
[89; теорема 0.7]), что $C^{n}\left(t_{1}, \ldots, t_{m}\right)$ имеет в точности $m$ вершин $\boldsymbol{x}\left(t_{i}\right)$, комбинаторный тип циклического многогранника не зависит от конкретного выбора параметров $t_{1}, \ldots, t_{m}$ и $C^{n}\left(t_{1}, \ldots, t_{m}\right)$ является смежностным симплициальным $n$-многогранником. Мы будем обозначать комбинаторньй циклический $n$-многогранник с $m$ вершинами через $C^{n}(m)$.

Пусть $P$ - простой $n$-многогранник. Обозначим через $f_{i}$ число его граней коразмерности $(i+1)$ (т.е. размерности $(n-i-1))$. В частности, $f_{0}$ есть число гиперграней многогранника $P$, которое мы будем обозначать через $m(P)$ или просто $m$.

ОПРЕДЕЛЕНИЕ 1.1.10. Целочисленный вектор $\boldsymbol{f}(P)=\left(f_{0}, \ldots, f_{n-1}\right)$ назьвается $f$-вектором простого многогранника $P$. Целочисленный вектор $\left(h_{0}, h_{1}, \ldots, h_{n}\right)$, компоненты которого определяются из уравнения

$$
h_{0} t^{n}+\cdots+h_{n-1} t+h_{n}=(t-1)^{n}+f_{0}(t-1)^{n-1}+\cdots+f_{n-1},
$$

назьвается $h$-вектором многогранника $P$. Наконец, целочисленный вектор $\left(g_{0}, g_{1}\right.$, $\left.\ldots, g_{\left[\frac{n}{2}\right]}\right)$, где $g_{0}=1, g_{i}=h_{i}-h_{i-1}, i>0$, назьвается $g$-вектором многогранника $P$.

Мы также полагаем $f_{-1}=1$, что означает, что сам многогранник считается гранњю коразмерности 0 . Для любого простого многогранника $f$-вектор и $h$-вектор выражаются друг через друга при помощи линейных соотношений, а именно,

$$
h_{k}=\sum_{i=0}^{k}(-1)^{k-i}\left(\begin{array}{c}
n-i \\
n-k
\end{array}\right) f_{i-1}, \quad f_{n-1-k}=\sum_{q=k}^{n}\left(\begin{array}{l}
q \\
k
\end{array}\right) h_{n-q}, \quad k=0, \ldots, n .
$$

В частности, $h_{0}=1$ и $h_{n}=(-1)^{n}\left(1-f_{0}+f_{1}+\cdots+(-1)^{n} f_{n-1}\right)$. По теореме Эйлера

$$
f_{0}-f_{1}+\cdots+(-1)^{n-1} f_{n-1}=1+(-1)^{n-1},
$$

что эквивалентно соотношению $h_{n}=1=h_{0}$. Для простых многогранников теорема Эйлера допускает следующее обобщение.

TеоРема 1.1.11 (соотношения Дена-Соммервилля). $h$-вектор любого простого многогранника симметричен, т.е.

$$
h_{i}=h_{n-i}, \quad i=0,1, \ldots, n .
$$

Имеется множество различных способов доказательства соотношений Дена-Соммервилля. Мы приводим доказательство, впервые появившееся в [18] и использующее соображения, напоминающие идеи из теории Морса.

ДОКАЗАТЕЛЬСТВО ТЕОРЕМЫ 1.1.11. Пусть $P^{n} \subset \mathbb{R}^{n}$ - простой многогранник. Выберем линейную функцию $\varphi: \mathbb{R}^{n} \rightarrow \mathbb{R}$ общего положения в том смысле, что она принимает различные значения на всех вершинах $P^{n}$. Для этой функции $\varphi$ найдется вектор $\boldsymbol{\nu} \in \mathbb{R}^{n}$ такой, что $\varphi(\boldsymbol{x})=\langle\boldsymbol{\nu}, \boldsymbol{x}\rangle$. Заметим, что вектор $\boldsymbol{\nu}$ не параллелен ни одному ребру многогранника $P^{n}$. Теперь мы будем рассматривать $\varphi$ как функцию высоты на $P^{n}$. При помощи $\varphi$ мы превратим 1-остов многогранника $P^{n}$ в ориентированный граф, направляя каждое ребро так, чтобы функция $\varphi$ возрастала вдоль него 
(напомним, что мы выбрали функцию $\varphi$ обшего положения). Для каждой вершины $v$ многогранника $P^{n}$ определим ее индекс ind $(v)$ как число ребер, входящих в $v$. Обозначим число вершин индекса $i$ через $I_{\nu}(i)$. Мы утверждаем, что $I_{\nu}(i)=h_{n-i}$. Действительно, каждая грань $P^{n}$ имеет единственную верхнюю вершину (максимум функции высоты $\varphi$, ограниченной на грань) и единственную нижнюю вершину (минимум $\varphi$ ). Пусть $F^{k}$ - некоторая $k$-грань и $v_{F}$ - ее верхняя вершина. Так как многогранник $P^{n}$ простой, в вершине $v_{F}$ сходятся в точности $k$ ребер из грани $F^{k}$, т.е. $\operatorname{ind}\left(v_{F}\right) \geqslant k$. С другой стороны, каждая вершина индекса $q \geqslant k$ является верхней вершиной для $\left(\begin{array}{l}q \\ k\end{array}\right)$ граней размерности $k$. Отсюда следует, что $f_{n-1-k}$ (число $k$-граней) может быть вычислено как

$$
f_{n-1-k}=\sum_{q \geqslant k}\left(\begin{array}{l}
q \\
k
\end{array}\right) I_{\nu}(q) .
$$

Второе из тождеств (8) показывает, что $I_{\nu}(q)=h_{n-q}$, что и требовалось. В частности, число $I_{\nu}(q)$ не зависит от $\nu$. С другой стороны, так как $\operatorname{ind}_{\nu}(v)=n-\operatorname{ind}_{-\nu}(v)$ для любой вершины $v$, мы имеем

$$
h_{n-q}=I_{\nu}(q)=I_{-\nu}(n-q)=h_{q} .
$$

Используя (8), соотношения Дена-Соммервилля можно переписать в терминах $f$-вектора в следующем виде:

$$
f_{k-1}=\sum_{j=k}^{n}(-1)^{n-j}\left(\begin{array}{l}
j \\
k
\end{array}\right) f_{j-1}, \quad k=0,1, \ldots, n .
$$

Соотношения Дена-Соммервилля были получены Деном для $n \leqslant 5$ в 1905 г. и Соммервиллем в общем случае в 1927 г. (см. [78]) в виде, аналогичном (10).

ПримеР 1.1.12. Пусть $P_{1}^{n_{1}}$ и $P_{2}^{n_{2}}$ - два простых многогранника. Учитьвая, что любая грань многогранника $P_{1} \times P_{2}$ есть произведение некоторой грани $P_{1}$ на грань $P_{2}$, мы непосредственно из определения $f$-вектора получаем:

$$
f_{k}\left(P_{1} \times P_{2}\right)=\sum_{i=-1}^{n_{1}} f_{i}\left(P_{1}\right) f_{k-i-1}\left(P_{2}\right), \quad k=-1,0, \ldots, n_{1}+n_{2}-1 .
$$

Положим $h(P ; t)=h_{0}+h_{1} t+\cdots+h_{n} t^{n}$. Тогда из последней формулы согласно $(7)$ вытекает соотношение

$$
h\left(P_{1} \times P_{2} ; t\right)=h\left(P_{1} ; t\right) h\left(P_{2} ; t\right) .
$$

ПримеР 1.1.13. Выразим $f$-вектор и $h$-вектор связной суммы $P^{n} \# Q^{n}$ в терминах $f$-векторов и $h$-векторов многогранников $P^{n}$ и $Q^{n}$. Как следует из конструкции 1.1.7,

$$
\begin{gathered}
f_{i}\left(P^{n} \# Q^{n}\right)=f_{i}\left(P^{n}\right)+f_{i}\left(Q^{n}\right)-\left(\begin{array}{c}
n \\
i+1
\end{array}\right), \quad i=0,1, \ldots, n-2 ; \\
f_{n-1}\left(P^{n} \# Q^{n}\right)=f_{n-1}\left(P^{n}\right)+f_{n-1}\left(Q^{n}\right)-2 \\
\text { (заметим, что } \left.\left(\begin{array}{c}
n \\
i+1
\end{array}\right)=f_{i}\left(\Delta^{n-1}\right)\right) . \text { Далее в силу }(8) \\
h_{0}\left(P^{n} \# Q^{n}\right)=h_{n}\left(P^{n} \# Q^{n}\right)=1 ; \\
h_{i}\left(P^{n} \# Q^{n}\right)=h_{i}\left(P^{n}\right)+h_{i}\left(Q^{n}\right), \quad i=1,2, \ldots, n-1 .
\end{gathered}
$$

Таким образом, $h_{i}, i=1, \ldots, n-1$, задают целочисленные функции на множестве простых многогранников, линейные относительно связной суммы. 
ПроБлема 1.1.14. Описать все челочисленнье функиии на множестве простых многогранников, линейные относительно связной суммы.

Для симплициального многогранника $P^{*}$ определим $f$-вектор как $\boldsymbol{f}\left(P^{*}\right)=\left(f_{0}, f_{1}\right.$, $\left.\ldots, f_{n-1}\right)$, где $f_{i}$ есть число $i$-граней $\left(i\right.$-симплексов) $P^{*}$. Тогда $h$-вектор $h\left(P^{*}\right)=$ $\left(h_{0}, h_{1}, \ldots, h_{n}\right)$ определяется соотношением $(7)$. Заметим, что если $P^{*}$ является симплициальным многогранником, полярным к простому многограннику $P$, то $f_{i}\left(P^{*}\right)=$ $f_{i}(P)$. Отсюда вытекает, что соотношения Дена-Соммервилля имеют место и для симплициальных многогранников.

ПримеР 1.1.15. Пусть $P^{*}-q$-смежностньй симплициальный $n$-многогранник. Тогда $f_{k-1}\left(P^{*}\right)=\left(\begin{array}{c}m \\ k\end{array}\right), k \leqslant q$, где $m=f_{0}\left(P^{*}\right)$ - число вершин. Из (8) мы выводим

$$
h_{k}\left(P^{*}\right)=\sum_{i=0}^{k}(-1)^{k-i}\left(\begin{array}{c}
n-i \\
k-i
\end{array}\right)\left(\begin{array}{c}
m \\
i
\end{array}\right)=\left(\begin{array}{c}
m-n+k-1 \\
k
\end{array}\right), \quad k \leqslant q
$$

(последнее равенство получается вычислением коэффищиента при $t^{k}$ в обеих частях тождества $\left.\frac{1}{(1+t)^{n-k+1}}(1+t)^{m}=(1+t)^{m-n+k-1}\right)$. Предположим, что $P^{*}$ не является симплексом. Тогда $m>n+1$, и из (12) следует, что $h_{0}<h_{1}<\cdots<h_{q}$. Как показывают соотношения Дена-Соммервилля, в этом случае имеет место неравенство $q \leqslant\left[\frac{n}{2}\right]$.

Возникает естественный вопрос: какие целочисленные векторы могут быть $f$-векторами простых (или, эквивалентно, симплициальных) многогранников? Соотношения Дена-Соммервилля дают необходимое условие.

ПРЕДЛОЖЕНИЕ 1.1.16 [52]. Соотношения Дена-Соммервилля являются наиболее общими линейными уравнениями, которым удовлетворяют $f$-векторы всех простых (или симплициальных) многогранников.

ДокАЗАТЕльство. В отличие от [52], мы будем использовать $h$-векторы. Достаточно показать, что аффинная оболочка $h$-векторов $\left(h_{0}, h_{1}, \ldots, h_{n}\right)$ простых $n$-многогранников представляет собой $\left[\frac{n}{2}\right]$-мерную плоскость. Положим $Q_{k}:=\Delta^{k} \times \Delta^{n-k}$, $k=0,1 \ldots,\left[\frac{n}{2}\right]$. Так как $\boldsymbol{h}\left(\Delta^{k}\right)=1+t+\cdots+t^{k}$, из (11) мы получаем

$$
\boldsymbol{h}\left(Q_{k}\right)=\frac{1-t^{k+1}}{1-t} \cdot \frac{1-t^{n-k+1}}{1-t} .
$$

Отсюда вытекает, что $\boldsymbol{h}\left(Q_{k+1}\right)-\boldsymbol{h}\left(Q_{k}\right)=t^{k+1}+\cdots, k=0,1, \ldots,\left[\frac{n}{2}\right]-1$. Следовательно, векторы $\boldsymbol{h}\left(Q_{k}\right), k=0,1 \ldots,\left[\frac{n}{2}\right]$, аффиннно независимы.

Мы отметим также, что соотношение (9) является единственным линейньм соотношением, которому удовлетворяют векторы чисел граней всех выпуклых многогранников.

Условия, характеризуюшие $f$-векторы простых (или симплициальных) многогранников, в настоящее время известные как $g$-теорем $а$, были высказаны в качестве гипотезы Макмюлленом [58] в 1970 г. и доказаны Стенли [80] (необходимость) и Биллерой, Ли [13] (достаточность) в 1980 г. Кроме соотношений Дена-Соммервилля, $g$-теорема содержит два набора неравенств, один из которых состоит из линейных неравенств, 
а другой - из нелинейных. Для того чтобы сформулировать $g$-теорему полностью, нам понадобится следующая конструкция. Для любых двух натуральных чисел $a, i$ существует единственное биномиальное $i$-разложсение числа $a$ вида

$$
a=\left(\begin{array}{c}
a_{i} \\
i
\end{array}\right)+\left(\begin{array}{c}
a_{i-1} \\
i-1
\end{array}\right)+\cdots+\left(\begin{array}{c}
a_{j} \\
j
\end{array}\right),
$$

где $a_{i}>a_{i-1}>\cdots>a_{j} \geqslant i \geqslant 1$. Определим

$$
a^{\langle i\rangle}=\left(\begin{array}{c}
a_{i}+1 \\
i+1
\end{array}\right)+\left(\begin{array}{c}
a_{i-1}+1 \\
i
\end{array}\right)+\cdots+\left(\begin{array}{c}
a_{j}+1 \\
j+1
\end{array}\right), \quad 0^{\langle i\rangle}=0
$$

ТЕОРема 1.1 .17 ( $g$-теорема). Целочисленный вектор $\left(f_{0}, f_{1}, \ldots, f_{n-1}\right)$ является $f$-вектором некоторого простого $n$-многогранника тогда и только тогда, когда соответствующая последовательность $\left(h_{0}, \ldots, h_{n}\right)$, определяемая (7), удовлетворяет следующим трем условиям:

(а) $h_{i}=h_{n-i}, i=0, \ldots, n$ (соотношения Дена-Соммервилля);

(б) $h_{0} \leqslant h_{1} \leqslant \cdots \leqslant h_{\left[\frac{n}{2}\right]}$, m.e. $g_{i} \geqslant 0, i=0,1, \ldots,\left[\frac{n}{2}\right]$.

(в) $h_{0}=1, h_{i+1}-h_{i} \leqslant\left(h_{i}-h_{i-1}\right)^{\langle i\rangle}$, m.e. $g_{i+1} \leqslant g_{i}^{\langle i\rangle}, i=1, \ldots,\left[\frac{n}{2}\right]-1$.

ЗАмечАниЕ. Очевидно, те же самые условия характеризуют $f$-векторы $c$ ммплиииальных многогранников.

Последовательность целых чисел $\left(k_{0}, k_{1}, \ldots, k_{r}\right)$, удовлетворяюшая условиям $k_{0}=1$ и $0 \leqslant k_{i+1} \leqslant k_{i}^{\langle i\rangle}, i=1, \ldots, r-1$, назьвается $M$-вектором (по имени М. Маколея). Условия (б) и (в) из $g$-теоремы означают, что $g$-вектор $\left(g_{0}, g_{1}, \ldots, g\left[\frac{n}{2}\right]\right)$ простого $n$-многогранника является $M$-вектором. С другой стороны, понятие $M$-вектора возникает в следующем классификационном результате из коммутативной алгебры.

ТЕОРема 1.1 .18 (Маколей). Последовательность $\left(k_{0}, k_{1}, \ldots, k_{r}\right)$ является $M$ вектором тогда и только тогда, когда существует градуированная коммутативная алгебра $A=A^{0} \oplus A^{2} \oplus \cdots \oplus A^{2 r}$ над полем $\mathbf{k}=A^{0}$, порожсденная (как алгебра) әлементами степени 2 , такая, что размерность $2 i$-й градуированной компоненты А равна $k_{i}$, m.е. $\operatorname{dim}_{\mathbf{k}} A^{2 i}=k_{i}, i=1, \ldots, r$.

Доказательство можно найти в [79].

Для доказательства достаточности $g$-теоремы Биллерой и Ли была предложена замечательная комбинаторно-геометрическая конструкция, позволяющая построить симплициальньй многогранник с любой наперед заданной $M$-последовательностью в качестве $g$-вектора. Доказательство Стенли условия необходимости в $g$-теореме (т.е. утверждения о том, что $g$-вектор простого многогранника является $M$-вектором) основывается на глубоких результатах алгебраической геометрии: сильной теореме Лефшеца для когомологий торических многообразий. Мы приводим идеи доказательства Стенли в $§ 2.1$. С 1995 г. появилось несколько более элементарных комбинаторных доказательств $g$-теоремы. Первое элементарное доказательство Макмюллена [59], использующее вместо алгебры когомологий торического многообразия понятие алгебры многогранника, было, тем не менее, достаточно сложным. Недавно В. А. Тимориным [86] было предложено более простое элементарное доказательство 
$g$-теоремы, которое основано на интерпретации алгебры многогранника Макмюллена как алгебры дифференциальных операторов (с постоянными коэффициентами), принимающих нулевое значение на многочлене облема многогранника.

Как вытекает из результатов [13], условие (б) из $g$-теоремы, т.е. неравенства

$$
h_{0} \leqslant h_{1} \leqslant \cdots \leqslant h_{\left[\frac{n}{2}\right]}
$$

дает наиболее обшие линейные неравенства, которьм удовлетворяют $f$-векторы всех простых (или симплициальных) многогранников. Эти неравенства в настояшее время известны как Обобщенная Теорема о Нижней Гранище (ОТНГ) для простых (симплициальных) многогранников. В течение последних двух десятилетий было получено множество результатов в направлении обобшения соотношений Дена-Соммервилля, ОТНГ и $g$-теоремы на более широкий класс объектов, чем симплициальные многогранники. Тем не менее, здесь остается большое количество интригуюших открытых проблем. Некоторые из них приведены в обзорной статье Стенли [83]. В настоящей работе мы также рассматриваем некоторые связанные с этим вопросы (см. комментарии в следуюшем параграфе).

Имеет место следующее важное следствие из $g$-теоремы.

Теорема 1.1.19 (Теорема о Верхней Границе (ТВГ) для симплициальных многогранников [89; теорема 8.23, следствие 8.38]). Циклический многогранник $C^{n}(m)$ (пример 1.1.9) имеет максимальное число $i$-граней, $2 \leqslant i \leqslant n-1$, среди всех симплициальных п-многогранников $P^{*}$ с $m$ вершинами. Таким образом, если $f_{0}\left(P^{*}\right)=m, m o$

$$
f_{i}\left(P^{*}\right) \leqslant f_{i}\left(C^{n}(m)\right), \quad 2 \leqslant i \leqslant n-1 .
$$

Заметим, что $f_{i}\left(C^{n}(m)\right)=\left(\begin{array}{c}m \\ i+1\end{array}\right)$ при $0 \leqslant i<\left[\frac{n}{2}\right]$. Последняя теорема была высказана в качестве гипотезы Мотцкином в 1957 г. Она была доказана Макмюлленом в 1970 г., что дало ему основание высказать в качестве гипотезы $g$-теорему. Макмюлленом было также показано, что ТВГ эквивалентна следующим неравенствам для $h$-вектора $\boldsymbol{h}\left(P^{*}\right)=\left(h_{0}, h_{1}, \ldots, h_{n}\right)$ :

$$
h_{i}\left(P^{*}\right) \leqslant\left(\begin{array}{c}
m-n+i-1 \\
i
\end{array}\right), \quad 0 \leqslant i \leqslant\left[\frac{n}{2}\right]
$$

(сравните это с примером 1.1.15).

Завершая изложение необходимых сведений о многогранниках, мы введем алгебраический инвариант (комбинаторного) простого многогранника, который будет играть важную роль в нашем обзоре. Пусть $P$ - простой $n$-многогранник с $m$ гипергранями $F_{1}, \ldots, F_{m}$. Зафиксируем некоторое коммутативное кольцо $\mathbf{k}$ с единицей. Пусть $\mathbf{k}\left[v_{1}, \ldots, v_{m}\right]$ - алгебра многочленов над $\mathbf{k}$ от $m$ переменных. Мы превратим ее в градуированную алгебру, полагая $\operatorname{deg}\left(v_{i}\right)=2$.

ОПРЕДЕЛЕНИЕ 1.1.20. Кольцом граней (или кольцом Стенли-Райснера) простого многогранника $P$ назьвается фактор-кольцо

$$
\mathbf{k}(P)=\mathbf{k}\left[v_{1}, \ldots, v_{m}\right] / \mathscr{I}_{P}
$$

где $\mathscr{I}_{P}$ - идеал, порожденньй всеми мономами $v_{i_{1}} v_{i_{2}} \cdots v_{i_{s}}, i_{1}<\cdots<i_{s}$, такими, что $F_{i_{1}} \cap \cdots \cap F_{i_{s}}=\varnothing$ в $P$. 
Очевидно, $\mathbf{k}(P)$ является градуированной $\mathbf{k}$-алгеброй.

Заметим, что кольцо Стенли-Райснера, а также $f$ - и $h$-векторы, являются инвариантами комбинаторного простого многогранника: они зависят только от решетки граней и не зависят от конкретной геометрической реализации.

§ 1.2. Симплициальные комплексы: топология и комбинаторика. Обозначим через $[m]$ множество индексов $\{1, \ldots, m\}$. Для любого подмножества $I \subset[m]$ будем обозначать число его элементов через \#I.

ОПРЕДЕЛЕНИЕ 1.2.1. (Абстрактным) симплициальным комплексом на множестве $[m]$ называется набор $K=\{I\}$ подмножеств множества $[m]$ такой, что для любого $I=\left\{i_{1}, \ldots, i_{k}\right\} \in K$ все подмножества $I$ (включая $\varnothing$ ) также принадлежат $K$. Подмножества $I \in K$ называются (абстрактными) симплексами комплекса $K$.

Аналогичным образом определяются симплищиальные комплексы на произвольном множестве $\mathscr{S}$. Одноэлементные подмножества из $K$ называются вериинами комплекса $K$. Если $K$ содержит все одноэлементные подмножества множества $[m]$, то говорят, что $K$ есть симплициальный комплекс на множестве вериин $[m]$. Размерность абстрактного симплекса $I=\left\{i_{1}, \ldots, i_{k}\right\} \in K$ равна числу его элементов минус один, т.е. $\operatorname{dim} I=\# I-1$. Размерность абстрактного симплициального комплекса полагается равной максимальной размерности его симплексов.

ОПРЕДЕЛЕНИЕ 1.2.2. Геометрическим симплициальным комплексом (или полиэдром) назьвается подмножество $\mathscr{P} \subset \mathbb{R}^{n}$, представленное в виде объединения симплексов произвольной размерности таким образом, что пересечение любых двух симплексов является гранью каждого из них (под симплексами мы здесь подразумеваем выпуклые многогранники из примера 1.1.3).

Далее мы будем использовать обозначение $\Delta^{m-1}$ как для абстрактного симплекса (т.е. симплициального комплекса, состоящего из всех подмножеств множества $[m]$ ), так и для соответствуюшего полиэдра.

ЗАмЕчАниЕ. Понятие полиэдра из определения 1.1.2 отличается от понятия из предыдущего определения. Первое значение термина “полиэдр" (т.е. "неограниченный многогранник") принято в выпуклой геометрии, в то время как второе значение (т.е. "геометрический симплициальньй комплекс") используется в комбинаторной топологии. Так как оба значения стали классическими в соответствуюших областях, мы стали менять их названия. В этом обзоре под “полиэдром" мы будем, как правило, понимать геометрический симплициальный комплекс. "Неограниченные многогранники" будут у нас называться "выпуклыми полиэдрами”. В любом случае из контекста всегда будет ясно, какой “полиэдр" имеется ввиду.

В настояшем обзоре рассматриваются только конечные геометрические симплициальные комплексы (полиэдры). Размерность полиэдра равна максимальной размерности его симплексов. Хорошо известно [72], что любой $n$-мерный абстрактный симплициальньй комплекс $K$ допускает геометрическую реализацию $|K|$ в качестве $n$-мерного полиэдра в пространстве $\mathbb{R}^{2 n+1}$ (абстрактные симплексы комплекса $K$ соответствуют (многогранным) симплексам полиэдра $|K|$ ). Геометрическая реализация $|K|$ абстрактного симплициального комплекса $K$ единственна с точностью до кусочно-линейного гомеоморфизма. 
Конструкция 1.2.3. Геометрическую реализацию в $m$-мерном пространстве симплициального комплекса $K$ на множестве вершин $[m]$ можно построить следующим образом. Пусть $\boldsymbol{e}_{i}$ обозначает $i$-й единичный координатный вектор пространства $\mathbb{R}^{m}$. Для каждого подмножества $I \subset[m]$ обозначим через $\Delta_{I}$ вьпуклую оболочку векторов $\boldsymbol{e}_{i}$ с $i \in I$. Очевидно, $\Delta_{I}$ является (геометрическим) симплексом. Тогда мы имеем

$$
|K|=\bigcup_{I \in K} \Delta_{I} \subset \mathbb{R}^{m} .
$$

Симплиииальным отображсением $f:\left|K_{1}\right| \rightarrow\left|K_{2}\right|$ двух полиэдров называетсяпроизвольное отображение множества вершин полиэдра $\left|K_{1}\right|$ в множество вершин $\left|K_{2}\right|$, продолженное линейно на симплексах из $\left|K_{1}\right|$ до отображения всего полиэдра $\left|K_{1}\right|$. Полиэдр $\left|K^{\prime}\right|$ называется подразделением полиэдра $|K|$, если каждый симплекс из $|K|$ является объединением конечного числа симплексов из $\left|K^{\prime}\right|$. Кусочно-линейнылм отображсением (или $P L$-отображсением) назьвается отображение $f:\left|K_{1}\right| \rightarrow\left|K_{2}\right|$, которое является симплициальным отображением некоторого подразделения полиэдра $\left|K_{1}\right|$ в некоторое подразделение $\left|K_{2}\right|$. Изложение основ кусочно-линейной топологии можно найти в [75].

ПримеР 1.2.4 (ассоциированный симплициальный комплекс). Пусть $K$ - симплициальный комплекс на множестве $[m]$. Предположим, что $K$ отличен от $(m-1)$-симплекса. Определим

$$
\widehat{K}:=\{I \subset[m]:[m] \backslash I \notin K\} .
$$

Легко видеть, что $\widehat{K}$ является симплициальным комплексом на множестве $[m]$. Он назьвается симплициальным комплексом, ассоциированным с $K$.

Конструкция 1.2.5 (соединение (джойн) симплициальных комплексов). Пусть $K_{1}, K_{2}$ - симплициальные комплексы на множествах $\left[m_{1}\right]$ и $\left[m_{2}\right]$ соответственно. Отождествим $\left[m_{1}\right] \cup\left[m_{2}\right]$ с $\left[m_{1}+m_{2}\right]$. Соединением (джойном) комплексов $K_{1}$ и $K_{2}$ называется симплициальньй комплекс

$$
K_{1} * K_{2}:=\left\{I \subset\left[m_{1}+m_{2}\right]: I=I_{1} \cup I_{2}, I_{1} \in K_{1}, I_{2} \in K_{2}\right\}
$$

на множестве $\left[m_{1}+m_{2}\right]$.

ПримеР 1.2.6. 1. Если $K_{1}=\Delta^{m_{1}-1}, K_{2}=\Delta^{m_{2}-1}$, то $K_{1} * K_{2}=\Delta^{m_{1}+m_{2}-1}$.

2. Симплициальный комплекс $\Delta^{0} * K$ (соединение $K$ с точкой) назьвается конусом над $K$ и обозначается cone $(K)$.

3. Пусть $S^{0}$ - симплициальньй комплекс, представляющий собой несвязное объединение двух точек. Тогда $S^{0} * K$ называется надстройкой над $K$ и обозначается $\Sigma K$.

Геометрическая реализация комплекса cone $(K)$ (комплекса $\Sigma K$ ) представляет собой топологический конус (надстройку) над $|K|$.

Барицентрическим подразделением абстрактного симплициального комплекса $K$ назьвается симплициальный комплекс $\operatorname{bs}(K)$ на множестве симплексов комплекса $K$ такой, что $\left\{I_{1}, \ldots, I_{r}\right\} \in \operatorname{bs}(K)$, если и только если $I_{1} \subset I_{2} \subset \cdots \subset I_{r}$ (после возможного переупорядочивания). Барицентром (вьпуклого) симплекса $\Delta^{n} \in \mathbb{R}^{n}$ 
с вершинами $v_{1}, \ldots, v_{n+1}$ называется точка $\mathrm{bc}\left(\Delta^{n}\right)=\frac{1}{n+1}\left(v_{1}+\cdots+v_{n+1}\right) \in \Delta^{n}$. Баричентрическим подразделением полиэдра $\mathscr{P}$ называется полиэдр $\operatorname{bs}(\mathscr{P})$, определяемьй следуюшим образом. Вершины полиэдра $\operatorname{bs}(\mathscr{P})$ суть барищентры симплексов всех размерностей полиэдра $\mathscr{P}$. Множество вершин $\left\{\mathrm{bc}\left(\Delta_{1}^{i_{1}}\right), \ldots, \mathrm{bc}\left(\Delta_{r}^{i_{r}}\right)\right\}$ порождает симплекс полиэдра $\operatorname{bs}(\mathscr{P})$ тогда и только тогда, когда $\Delta_{1}^{i_{1}} \subset \cdots \subset \Delta_{r}^{i_{r}}$ в $\mathscr{P}$. Очевидно, мы имеем $|\operatorname{bs}(K)|=\operatorname{bs}(|K|)$ для любого абстрактного симплициального комплекса $K$.

ПРИМЕР 1.2.7 (порядковый комплекс частично упорядоченного множества). Пусть $(\mathscr{S}, \prec)$ - произвольное частично упорядоченное множество. Определим $K(\mathscr{S})$ как множество всех цепей $x_{1} \prec x_{2} \prec \cdots \prec x_{k}, x_{i} \in \mathscr{S}$. Легко видеть, что $K(\mathscr{S})$ - симплициальный комплекс. Он называется порядковым комплексом частично упорядоченного множества $(\mathscr{S}, \prec)$. В частности, если $(\mathscr{S}, \varsubsetneqq)$ - частично упорядоченное множество граней (решетка граней) некоторого симплициального комплекса $K$, то $K(\mathscr{S})-$ барицентрическое подразделение $K$.

Недостающей гранью симплищиального комплекса $K$ на множестве $[m]$ назьвается подмножество $I \subset[m]$ такое, что $I \notin K$, но любое собственное подмножество множества $I$ является симплексом комплекса $K$. Флаговым комплексом назьвается симплициальньй комплекс, для которого любая недостающая грањь состоит из двух элементов. Порядковые комплексы частично упорядоченных множеств (в частности, барищентрические подразделения) являются флаговыми комплексами ввиду условия транзитивности.

Для любого подмножества $I \subset[m]$ обозначим через $K_{I}$ подкомплекс комплекса $K$, состоящий из всех симплексов $J \in K$ таких, что $J \subset I$. Линком симплекса $I \in K$ назьвается подкомплекс $\operatorname{link}_{K} I \subset K$, состоящий из всех симплексов $J \in K$ таких, что $I \cup J \in K$ и $I \cap J=\varnothing$. Для любой вершины $\{i\} \in K$ конус над комплексом $\operatorname{link}_{K}\{i\}$ (с вершиной $\{i\}$ ) естественным образом является подкомплексом в $K$, который назьвается звездой вершины $\{i\}$ и обозначается $\operatorname{star}_{K}\{i\}$. Полиэдр $\left|\operatorname{star}_{K}\{i\}\right|$ состоит из всех (многогранных) симплексов полиэдра $|K|$, содержаших $\{i\}$. Если из контекста ясно, какой комплекс $K$ имеется в виду, то мы будем использовать обозначения link $I$ и $\operatorname{star}\{i\}$ вместо $\operatorname{link}_{K} I$ и $\operatorname{star}_{K}\{i\}$ соответственно. Определим core $[m]=$ $\{i \in[m]: \operatorname{star}\{i\} \neq K\}$. Ядром симплициального комплекса $K$ называется подкомплекс core $K=K_{\text {core[m] }}$. Таким образом, ядро есть максимальньй подкомплекс, содержаший все вершины, звезды которых не совпадают с $K$.

ПРИМЕР 1.2.8. 1. $\operatorname{link}(\varnothing)=K$.

2. Пусть $K$ - симплекс на четырех вершинах $1,2,3,4$ и $I=\{1,2\}$. Тогда $\operatorname{link}(I)-$ подкомплекс, состоящий из двух вершин 3 и 4.

3. Пусть $K-$ конус над $K^{\prime}$ с вершиной $p$. Тогда $\operatorname{link}\{p\}=K^{\prime}, \operatorname{star}\{p\}=K$ и core $K \subset K^{\prime}$.

Симпличиальная $n$-мерная сфера - это симплициальньй комплекс, гомеоморфный $n$-мерной сфере $S^{n}$ (здесь и далее, говоря “симплициальньй комплекс $K$ гомеоморфен $X$ ”, мы подразумеваем, что геометрическая реализация $|K|$ гомеоморфна $X)$. Кусочно-линейной сферой (или $P L$-сферой) назьвается симплициальная сфера, которая кусочно линейно гомеоморфна границе симплекса. Граница симплициального $n$-многогранника является $(n-1)$-мерной $P L$-сферой. $P L$-сферы, получаемые таким 
образом, назьваются многогранныци сферами. Таким образом, мы имеем следующие вложенные классы комбинаторных объектов:

многогранные сферы $\subset P L$-сферы $\subset$ симплициальные сферы.

В размерности 2 все симплициальные сферы являются многогранньми (см., например, [89; теорема 5.8]). Тем не менее, в высших размерностях оба включения являются строгими. Первые примеры трехмерных $P L$-сфер, не являющихся многогранными , были найдены Грюнбаумом, и наименшшая из таких сфер имеет 8 вершин. Изложение этих примеров можно найти в [9]. Симплициальная сфера, не являющаяся $P L$-сферой, приведена ниже в примере 1.2.10.

Понятия $f$-вектора и $h$-вектора $(n-1)$-мерного симплициального комплекса $K^{n-1}$ вводятся так же, как и для симплициальных многогранников: $\boldsymbol{f}\left(K^{n-1}\right)=\left(f_{0}, f_{1}, \ldots\right.$, $\left.f_{n-1}\right)$, где $f_{i}$ - число $i$-мерных симплексов в $K^{n-1}$, и $\boldsymbol{h}\left(K^{n-1}\right)=\left(h_{0}, h_{1}, \ldots, h_{n}\right)$, где $h_{i}$ определяются из (7). Здесь мы также полагаем $f_{-1}=1$. Если $K^{n-1}=\partial P^{*}-$ граница симплициального $n$-многогранника $P^{*}$, то мы, очевидно, имеем $f\left(K^{n-1}\right)=$ $\boldsymbol{f}\left(P^{*}\right)$.

Так как $f$-вектор многогранной симплициальной сферы совпадает с $f$-вектором соответствуюшего (симплициального) многогранника, $g$-теорема (теорема 1.1.17) имеет место также и для многогранных сфер. Таким образом, естественно возникает вопрос, обобшается ли $g$-теорема на случай произвольной симплициальной сферы. Этот вопрос был поставлен Макмюлленом [58] как обобшение его гипотезы для симплициальных многогранников. После 1980 г., когда гипотеза Макмюллена была доказана Биллерой, Ли и Стенли, следуюшая проблема стала, возможно, главной открытой комбинаторно-геометрической проблемой, связанной с $f$-векторами симплициальных комплексов.

Проблема 1.2.9 ( $g$-гипотеза для симплициальных сфер). Верно ли, что g-теорема (теорема 1.1.17) имеет место для симплициальных сфер?

Эта гипотеза является открытой даже для $P L$-сфер. Заметим, что для доказательства гипотезы для симплициальных сфер необходимо проверить лишь что $g$-вектор является $M$-вектором. Если $g$-гипотеза верна, то она дает полное описание $f$-векторов симплициальных сфер.

Первая часть теоремы 1.1.17 (соотношения Дена-Соммервилля) известна для симплипиальных сфер (см. следствие 1.4.15 ниже). Первое неравенство $h_{0} \leqslant h_{1}$ из второй части $g$-теоремы эквивалентно неравенству $1 \leqslant f_{0}-n$, которое очевидно. Неравенство $h_{1} \leqslant h_{2}, n \geqslant 4$, эквивалентно условию $f_{1} \geqslant n f_{0}-\left(\begin{array}{c}n+1 \\ 2\end{array}\right)$ для числа ребер, которое также известно для симплициальных сфер (см. [10]; фактически, доказательство условия нижней границы для числа ребер симплициального многогранника проходит и для симплициальных сфер). Все вместе эти факты показывают, что $g$-гипотеза верна для симплициальных сфер размерности $\leqslant 4$. Вопрос о неравенстве $h_{2} \leqslant h_{3}(n \geqslant 6)$ из ОТНГ (второй части теоремы 1.1.17) остается открытым. В течение последних двух десятилетий было предпринято большое количество попыток доказательства $g$-гипотезы. Несмотря на то, что все эти попытки были неудачными, они привели к некоторым весьма интересным переформулировкам $g$-гипотезы. Мы лишь упомянем 
результаты Пахнера [67], [68], которые сводят $g$-гипотезу (для $P L$-сфер) к доказательству некоторых свойств бизвездныхх преобразований ${ }^{1}$, и результаты из [85], которые показывают, что эта гипотеза вытекает из остовной $r$-жсесткости ${ }^{2}$ симплициальной $(n-1)$-сферы для $r \leqslant\left[\frac{n}{2}\right]$. Как было показано независимо Калаи и Стенли [81; следствие 2.4], ОТНГ вьполнена для гранипы $n$-мерного шара, являюшегося подкомплексом граничного комплекса симплициального $(n+1)$-многогранника. При этом, однако, неизвестно, какие симплициальные комплексы могут появляться таким образом. Безуспешность многочисленных попыток доказательства $g$-гипотезы послужила основанием для Бьорнера и Люца организовать компютерный поиск контрпримеров [15]. Их компьютерная программа BISTELLAR позволила получить множество замечательных результатов о триангуляциях многообразий, однако контрпримеры к $g$-гипотезе обнаружены не были. Дополнительную информацию об истории $g$-теоремы и связанных с этим вопросах можно найти в [82], [83], [89; лекция 8].

Симплициальный комплекс $K$ называется симплициальнымм многообразием (или триангулированныц многообразием), если полиэдр $|K|$ является топологическим многообразием. Все многообразия, рассматриваемые в нашем обзоре, предполагаются компактными, связными и замкнутыми (если не оговорено противное). Если $K^{q}$ является симплициальным многообразием, то для любого непустого симплекса $I \in K^{q}$ подкомплекс $\operatorname{link}(I)$ имеет гомологии, как у $(q-\# I)$-сферы (см. теорему 1.2.11 ниже). Симплициальньй комплекс $K^{q}$ называется $q$-мерным $P L$-многообразием (или комбинаторным многообразием), если $\operatorname{link}(I)$ является $P L$-сферой размерности $(q-\# I)$ для любого непустого симплекса $I \in K^{q}$.

ЗАмечАниЕ. Если $K^{q}$ является $P L$-многообразием, то для каждой вершины $\{i\} \in K^{q}$ соответствуюшая $(q-1)$-мерная $P L$-сфера $\operatorname{link}\{i\}$ ограничивает открытую окрестность $U_{i}$, которая $P L$-гомеоморфна $q$-мерному шару. Так как каждая точка полиэдра $\left|K^{q}\right|$ содержится в $U_{i}$ для некоторого $i$, это определяет $P L$-атлас для $\left|K^{q}\right|$.

ПримеР 1.2.10 (симплициальная 5-мерная сфера, не являюшаяся $P L$-сферой). Пусть $S_{H}^{3}$-произвольная триангуляция гомологической, но не топологической, 3 -мерной сферы, т.е. неодносвязное симплициальное многообразие, имеюшее гомологии, как у 3 -мерной сферы $S^{3}$. Одним из примеров такого многообразия является $с ф е р а ~$ Пуанкаре $S O(3) / A_{5}$. В силу теоремы Кэннона [27] двойная надстройка $\Sigma^{2} S_{H}^{3}$ гомеоморфна сфере $S^{5}$. Тем не менее, $\Sigma^{2} S_{H}^{3}$ не может быть $P L$-сферой, так как $S_{H}^{3}$ является линком некоторого 1 -симплекса в $\Sigma^{2} S_{H}^{3}$.

Следующая теорема дает комбинаторную характеризацию симплициальных комплексов, которые являются симплициальными многообразиями размерности $\geqslant 5$, и обобшает упомянутый выше результат Кэннона.

ТЕОРема 1.2.11 (Эдвардс [38]). При $q \geqslant 5$ полиәдр симплициального комплекса $\mathrm{K}^{q}$ является топологическим многообразием размерности q тогда и только тогда, когда для любого непустого симплекса $I \in K^{q}$ подкомплекс $\operatorname{link} I$ имеет гомологии, как у $(q-\# I)$-мерной сферьи, и для любой вершины $\{i\} \in K$ подкомплекс $\operatorname{link}\{i\}$ является односвязным.

\footnotetext{
${ }^{1}$ В оригинале bistellar moves.

${ }^{2} \mathrm{~B}$ оригинале skeletal r-rigidity.
} 
Вопрос о том, какие топологические многообразия могут быть триангулированы, является фундаментальным в комбинаторной топологии. Любое гладкое многообразие может быть триангулировано по теореме Уитни. Все топологические 2- и 3-мерные многообразия также допускают триангуляцию (для 2-мерных многообразий это почти очевидно, а для 3-мерных см. [61]). Более того, так как линк произвольной вершины симплициального 3-мерного многообразия является 2-мерной сферой (а все 2-мерные сферы являются $P L$-сферами), все 2- и 3 -мерные многообразия являются $P L$-многообразиями. Однако, уже в размерности 4 сушествуют топологические многообразия, которые не допускают $P L$-триангуляции (например, "фальшивое" пространство $\mathbb{C} P^{2}$ Фридмана). Более того, существуют топологические 4 -мерные многообразия, которые вообще не могут быть триангулировань (например, топологическое 4-многообразие Фридмана с формой пересечения $E_{8}$ ). Оба примера описаны в [3]. В размерностях $\geqslant 5$ имеется следующая знаменитая комбинаторно-топологическая проблема.

ПроБлема 1.2.12 (гипотеза триангуляции). Верно ли, что любое топологическое многообразие размерности $\geqslant 5$ может быть триангулировано?

Другая хорошо известная проблема из $P L$-топологии связана с вопросом о единственности кусочно-линейной структуры на топологической сфере.

ПробЛема 1.2.13. Верно ли, что PL-многообразие, гомеоморфное топологической 4-сфере, является $P L$-сферой?

Четыре является единственной размерностью, в которой вопрос о единственности $P L$-структуры на топологической сфере остается открытым. В размерностях $\leqslant 3$ единственность была доказана в [61], а в размерностях $\geqslant 5$ она вытекает из результатов Кирби и Зибенманна [51]. В размерности 4 категория $P L$-многообразий эквивалентна гладкой категории, поэтому предыдушая проблема эквивалентна проблеме сушествования экзотических 4-сфер.

Дополнительную информацию о последних достижениях и открытых проблемах комбинаторной и $P L$-топологии можно найти в [62], [73].

§1.3. Симплициальные комплексы: коммутативная алгебра. Аппарат коммутативной алгебры находит широкие применения в комбинаторике симплишиальных комплексов и связанных с ними объектов. Основным инструментом для перевода комбинаторных результатов и проблем на алгебраический язык служит кольцо Стенли-Райснера симплициального комплекса. Этот подход был предложен Стенли в начале 1970 -х годов.

Напомним, что $\mathbf{k}\left[v_{1}, \ldots, v_{m}\right]$ обозначает градуированную алгебру многочленов над коммутативным кольцом $\mathbf{k}$ с единицей, $\operatorname{deg}\left(v_{i}\right)=2$.

ОПРЕДЕЛЕнИЕ 1.3.1. Кольцом граней (или кольцом Стенли-Райснера) симплициального комплекса $K$ с $m$ вершинами назьвается фактор-кольцо

$$
\mathbf{k}(K)=\mathbf{k}\left[v_{1}, \ldots, v_{m}\right] / \mathscr{I}_{K},
$$

где $\mathscr{I}_{K}$ - однородньй идеал, порожденньй мономами $v_{i_{1}} v_{i_{2}} \cdots v_{i_{s}}, i_{1}<\cdots<i_{s}$, такими, что $I=\left\{i_{1}, \ldots, i_{s}\right\}$ не является симплексом комплекса $K$. 
Заметим, что идеал $\mathscr{I}_{K}$ имеет базис, состоящий из мономов $v_{i_{1}} v_{i_{2}} \cdots v_{i_{s}}$ без квадратов таких, что $I=\left\{i_{1}, \ldots, i_{s}\right\}$ - недостающая грань комплекса $K$. Идеалы кольца многочленов, в которых можно выбрать базис из мономов, назьваются мономиальньц.ми.

ПРЕДЛОЖЕНИЕ 1.3.2. Любой мономиальный идеал кольца многочленов, имеющий базис из мономов без квадратов, имеет вид $\mathscr{I}_{K}$ для некоторого симплиииального комплекса $K$.

ДокАЗАТЕльство. Для любого подмножества $I=\left\{i_{1}, \ldots, i_{k}\right\} \subset[m]$ обозначим через $v_{I}$ моном $v_{i_{1}} \cdots v_{i_{k}}$. Пусть $\mathscr{I}$ - идеал кольца многочленов, имеющий базис из мономов без квадратов. Положим

$$
K=\left\{I \subset[m]: v_{[m] \backslash I} \in \mathscr{I}\right\}
$$

Тогда легко проверить, что $K$ - симплищиальньй комплекс и $\mathscr{I}=\mathscr{I}_{K}$.

Пусть $P$ - простой $n$-многогранник и $P^{*}$ - его полярньй симплициальньй многогранник. Обозначим через $K_{P}$ границу $P^{*}$. Тогда $K_{P}$ является многогранной симплищиальной $(n-1)$-сферой. Очевидно, кольцо граней многогранника $P$ из определения 1.1.20 совпадает с кольцом граней комплекса $K_{P}$ из определения 1.3.1: $\mathbf{k}(P)=\mathbf{k}\left(K_{P}\right)$.

Пусть $M=M^{0} \oplus M^{1} \oplus \cdots-$ градуированный k-модуль. Ряд

$$
F(M ; t)=\sum_{i=0}^{\infty}\left(\operatorname{dim}_{\mathbf{k}} M^{i}\right) t^{i}
$$

назьвается рядом Пуанкаре модуля $M$.

ЗАмЕчАниЕ. В алгебраической литературе ряд $F(M ; t)$ называется также рядом Гильберта или рядом Гильберта-Пуанкаре.

Следующая лемма устанавливает взаимосвязь между двумя комбинаторными инвариантами симплициального комплекса: кольцом граней и $f$-вектором (или $h$-вектором).

ЛЕмма 1.3.3 (Стенли [82; теорема II.1.4]). Ряд Пуанкаре кольца $\mathbf{k}\left(K^{n-1}\right)$ может быть вычислен как

$$
F\left(\mathbf{k}\left(K^{n-1}\right) ; t\right)=\sum_{i=-1}^{n-1} \frac{f_{i} t^{2(i+1)}}{\left(1-t^{2}\right)^{i+1}}=\frac{h_{0}+h_{1} t^{2}+\cdots+h_{n} t^{2 n}}{\left(1-t^{2}\right)^{n}}
$$

где $\left(f_{0}, \ldots, f_{n-1}\right)-f$-вектор и $\left(h_{0}, \ldots, h_{n}\right)-h$-вектор комплекса $K^{n-1}$.

Заметим, что второе тождество из леммы 1.3.3 является очевидным следствием формул (8). 
ПримеР 1.3.4. 1. Пусть $K=\Delta^{n}$ ( $n$-симплекс). Тогда $f_{i}=\left(\begin{array}{c}n+1 \\ i+1\end{array}\right)$ при $-1 \leqslant i \leqslant n$, $h_{0}=1$ и $h_{i}=0$ при $i>0$. Так как любое подмножество множества $[n+1]$ является симплексом в $\Delta^{n}$, мы имеем $\mathbf{k}\left(\Delta^{n}\right)=\mathbf{k}\left[v_{1}, \ldots, v_{n+1}\right]$. Таким образом, $F\left(\mathbf{k}\left(\Delta^{n}\right) ; t\right)=$ $\frac{1}{\left(1-t^{2}\right)^{n+1}}$, что согласуется с леммой 1.3 .3 .

2. Пусть $K$ - граница $n$-симплекса. Тогда $h_{i}=1, i=0,1, \ldots, n$, и $\mathbf{k}(K)=$ $\mathbf{k}\left[v_{1}, \ldots, v_{n+1}\right] /\left(v_{1} v_{2} \cdots v_{n+1}\right)$. Отсюда

$$
F(\mathbf{k}(K) ; t)=\frac{1+t^{2}+\cdots+t^{2 n}}{\left(1-t^{2}\right)^{n}} .
$$

Теперь предположим, что $\mathbf{k}$ - поле. Пусть $A$ - градуированная алгебра над $\mathbf{k}$. Размерностью Крулля алгебры $A$ (обозначается $\operatorname{Kd} A$ ) называется максимальное число алгебраически независимых элементов из $A$. Последовательность $\theta_{1}, \ldots, \theta_{n}$ из $n=\mathrm{Kd} A$ однородных элементов алгебры $A$ называется однородной системой параметров, если размерность Крулля фактор-алгебры $A /\left(\theta_{1}, \ldots, \theta_{n}\right)$ равна нулю. Эквивалентно, $\theta_{1}, \ldots, \theta_{n}$ является однородной системой параметров, если $n=\mathrm{Kd} A$ и $A$ является конечнопорожденным $\mathbf{k}\left[\theta_{1}, \ldots, \theta_{n}\right]$-модулем. Элементы однородной системы параметров автоматически алгебраически независимы.

ЛЕмма 1.3 .5 (лемма Нетера о нормализации). Для любой градуированной конечнопорожденной алгебры А существует однородная система параметров. Если поле $\mathbf{k}$ имеет нулевую характеристику, а алгебра А порождена әлементами степени два, то можно выбрать однородную систему параметров степени $\partial в a$.

Далее в этом параграфе мы предполагаем, что поле $\mathbf{k}$ имеет характеристику нуль. Последовательность $\theta_{1}, \ldots, \theta_{k}$ однородных элементов алгебры $A$ назьвается регулярной последовательностью, если $\theta_{i+1}$ не является делителем нуля в кольце $A /\left(\theta_{1}, \ldots, \theta_{i}\right)$ при $0 \leqslant i<k$ (т.е. умножение на $\theta_{i+1}$ задает мономорфизм алгебры $A /\left(\theta_{1}, \ldots, \theta_{i}\right)$ в себя). Эквивалентно, $\theta_{1}, \ldots, \theta_{k}$ является регулярной последовательностью, если и только если элементы $\theta_{1}, \ldots, \theta_{k}$ алгебраически независимы и $A$ является свободным. $\mathbf{k}\left[\theta_{1}, \ldots, \theta_{k}\right]$-модулем.

ЗАмечАниЕ. Регулярные последовательности можно определять также в градуированных алгебрах, не являюшихся конечнопорожденными, а также в алгебрах над кольцами без делителей нуля. Регулярные последовательности в градуированном кольце многочленов от бесконечного числа образующих $R\left[a_{1}, a_{2}, \ldots\right], \operatorname{deg} a_{i}=-2 i$, где $R$ - некоторое подкольцо поля рациональный чисел $\mathbb{Q}$, играют важную роль в алгебраической топологии при построении теории комплексных кобордизмов с коэффициентами, см. [54].

Любые две максимальные регулярные последовательности имеют одинаковую длину, которая называется глубиной алгебры $A$ и обозначается $\operatorname{depth} A$. Очевидно, $\operatorname{depth} A \leqslant \operatorname{Kd} A$.

ОПРеДЕЛЕниЕ 1.3.6. Алгебра $A$ назьвается алгеброй Коэна-Маколея, если в ней имеется регулярная последовательность $\theta_{1}, \ldots, \theta_{n}$ длины $n=\operatorname{Kd} A$.

Любая регулярная последовательность $\theta_{1}, \ldots, \theta_{n}$ длины $n=\mathrm{Kd} A$ является однородной системой параметров. Таким образом, $A$ является алгеброй Коэна-Маколея 
тогда и только тогда, когда существует однородная система параметров $\theta_{1}, \ldots, \theta_{n}$ такая, что $A$ является конечнопорожденным свободным $\mathbf{k}\left[\theta_{1}, \ldots, \theta_{n}\right]$-модулем. Если, кроме того, алгебра $A$ порождена элементами степени два, то можно выбрать регулярную последовательность $\theta_{1}, \ldots, \theta_{n}$ степени два. В этом случае для ряда Пуанкаре алгебры $A$ имеет место тождество

$$
F(A ; t)=\frac{F\left(A /\left(\theta_{1}, \ldots, \theta_{n}\right) ; t\right)}{\left(1-t^{2}\right)^{n}},
$$

где $F\left(A /\left(\theta_{1}, \ldots, \theta_{n}\right) ; t\right)=h_{0}+h_{1} t^{2}+\cdots$ - некоторый многочлен. Конечный вектор $\left(h_{0}, h_{1}, \ldots\right)$ назьвается $h$-вектором алгебры $A$.

Симплициальный комплекс $K^{n-1}$ назьвается комплексом Коэна-Маколея (надk), если его кольцо граней $\mathbf{k}(K)$ является алгеброй Коэна-Маколея. Очевидно, $\operatorname{Kd} \mathbf{k}(K)=n$. Лемма 1.3 .3 показывает, что $h$-вектор алгебры $\mathbf{k}(K)$ совпадает с $h$-вектором комплекса $K$.

ТЕОрема 1.3.7 (Стенли). Если $K^{n-1}$ является комплексом Коэна-Маколея, то $\boldsymbol{h}\left(K^{n-1}\right)=\left(h_{0}, \ldots, h_{n}\right)$ является $M$-вектором $($ см. $\S 1.2)$.

ДоКАЗАТЕльСТвО. Пусть $\theta_{1}, \ldots, \theta_{n}$ - регулярная последовательность элементов степени два в $\mathbf{k}(K)$. Тогда $A=\mathbf{k}(K) /\left(\theta_{1}, \ldots, \theta_{n}\right)$ является градуированной алгеброй, порожденной элементами степени два, и $\operatorname{dim}_{\mathbf{k}} A^{2 i}=h_{i}$. Тогда утверждение вытекает из теоремы 1.1.18.

Следующая фундаментальная теорема характеризует комплексы Коэна-Маколея.

Теорема 1.3.8 (Райснер [74]). Симплициальный комплекс K является комплексом Коэна-Маколея над $\mathbf{k}$ тогда и только тогда, когда для любого симплекса $I \in K($ включая $I=\varnothing)$ и любого $i<\operatorname{dim}(\operatorname{link} I)$ имеет место $\widetilde{H}_{i}(\operatorname{link} I ; \mathbf{k})=0$ (здесь $\widetilde{H}_{i}(\cdot ; \mathbf{k})$ обозначает $i$-ю приведенную группу гомологий с коэффициентами в $\mathbf{k})$.

В частности, симплициальные сферы являются комплексами Коэна-Маколея. Из теоремы 1.3.7 вытекает, что $h$-вектор симплициальной сферы является $M$-вектором. Этот факт позволил Стенли обобшить ТВГ (теорему 1.1.19) на случай симплициальных сфер.

СЛЕДСТВИЕ 1.3.9 (Стенли). Теорема о Верхней Граниче имеет место для симпличиальных сфер, т.е. если $\left(h_{0}, h_{1}, \ldots, h_{n}\right)$ - h-вектор симплициальной $(n-1)$-сферьц $K^{n-1}$ с т вериинами, то

$$
h_{i}\left(K^{n-1}\right) \leqslant\left(\begin{array}{c}
m-n+i-1 \\
i
\end{array}\right), \quad 0 \leqslant i \leqslant\left[\frac{n}{2}\right] .
$$

ДокАЗАТЕльство. Так как $\boldsymbol{h}\left(K^{n-1}\right)$ является $M$-вектором, сушествует градуированная алгебра $A=A^{0} \oplus A^{2} \oplus \cdots \oplus A^{2 n}$, порожденная элементами степени два, такая, что $\operatorname{dim}_{\mathbf{k}} A^{2 i}=h_{i}$ (теорема 1.1 .18 ). В частности, $\operatorname{dim}_{\mathbf{k}} A^{2}=h_{1}=m-n$. Так как алгебра $A$ порождена элементами из $A^{2}$, число $h_{i}$ не может превышать числа мономов степени $i$ от $(m-n)$ переменных. Последнее число есть в точности $\left(\begin{array}{c}m-n+i-1 \\ i\end{array}\right)$. 
$\S$ 1.4. Гомологические свойства колец граней (колец Стенли-Райснера). Начнем с обзора некоторых понятий гомологической алгебры. В этом параграфе, если не оговорено противное, модулем мы называем конечнопорожденный градуированный $\mathbf{k}\left[v_{1}, \ldots, v_{m}\right]$-модуль, $\operatorname{deg} v_{i}=2$.

Конечной свободной резольвентой модуля $M$ назьвается точная последовательность

$$
0 \longrightarrow R^{-h} \stackrel{d}{\longrightarrow} R^{-h+1} \stackrel{d}{\longrightarrow} \cdots \longrightarrow R^{-1} \stackrel{d}{\longrightarrow} R^{0} \stackrel{d}{\longrightarrow} M \longrightarrow 0,
$$

где $R^{-i}$ - конечнопорож денные свободные модули и отображения $d$ сохраняют градуировку. Минимальное число $h$, для которого сушествует свободная резольвента (14), назьвается гомологической размерностью модуля $M$ и обозначается hd $M$. В силу теоремы Гильберта о сизигиях мы имеем hd $M \leqslant m$. Резольвента (14) определяет свободный биградуированный дифференциальныи модуль $[R, d]$, где $R=\bigoplus R^{-i, j}$, $R^{-i, j}:=\left(R^{-i}\right)^{j}\left(j\right.$-я градуированная компонента свободного модуля $\left.R^{-i}\right)$. Когомологии модуля $[R, d]$ равны нулю в ненулевых размерностях, а $H^{0}[R, d]=M$. Обратно, свободный биградуированный дифференциальньй модуль $\left[R=\bigoplus_{i, j \geqslant 0} R^{-i, j}, d\right.$ : $\left.R^{-i, j} \rightarrow R^{-i+1, j}\right]$ с $H^{0}[R, d]=M$ и $H^{-i}[R, d]=0$ при $i>0$ определяет свободную резольвенту (14) с $R^{-i}:=R^{-i, *}=\bigoplus_{j} R^{-i, j}$.

ЗАмЕчАниЕ. По причинам, указанным ниже, мы нумеруем члены свободной резольвенты неположительны.ми числами, преврашая ее в коцепной комплекс.

Ряд Пуанкаре модуля $M$ можно вычислить при помоши любой свободной резольвенты (14).

ТеОРема 1.4.1. Пусть свободныц й $\mathbf{k}\left[v_{1}, \ldots, v_{m}\right]$-модуль $R^{-i}$ в (14) порожден әлементами степеней $d_{1 i}, \ldots, d_{q_{i} i}$, әде $q_{i}=\operatorname{rank} R^{-i}, i=1, \ldots, h$. Тогда

$$
F(M ; t)=\left(1-t^{2}\right)^{-m} \sum_{i=0}^{h}(-1)^{i}\left(t^{d_{1 i}}+\cdots+t^{d_{q_{i} i}}\right) .
$$

ПримеР 1.4.2 (минимальная резольвента). По разным причинам часто бьвает удобным иметь резольвенту (14), в которой каждый член $R^{-i}$ имеет наименший возможный ранг. Следующее определение взято из работы Адамса [2]. Пусть $M, M^{\prime}-$ два модуля. Положим $\mathscr{J}(M)=v_{1} M+v_{2} M+\cdots+v_{m} M \subset M$. Отображение $f: M \rightarrow M^{\prime}$ назьвается минимальныцм, если $\operatorname{Ker} f \subset \mathscr{J}(M)$. Резольвента (14) назьвается минимальной, если все отображения $d$ минимальны. Тогда легко видеть, что все модули $R^{-i}$ имеют наименьший возможный ранг.

Минимальная резольвента строится следуюшим образом. Выберем вначале минимальный набор образующих $a_{1}, \ldots, a_{k_{0}}$ модуля $M$ и возьмем в качестве $R^{0}$ свободньй $\mathbf{k}\left[v_{1}, \ldots, v_{m}\right]$-модуль с $k_{0}$ образуюшими. Затем выберем минимальный набор образуюших $a_{1}, \ldots, a_{k_{1}}$ в ядре естественного эпиморфизма $R^{0} \rightarrow M$ и возьмем в качестве $R^{-1}$ свободный $\mathbf{k}\left[v_{1}, \ldots, v_{m}\right]$-модуль с $k_{1}$ образующими, и так далее. На $i$-м шаге мы выбираем минимальньй набор образуюших в ядре уже построенного отображения $d: R^{-i+1} \rightarrow R^{-i+2}$ и берем в качестве $R^{-i}$ свободный модуль с соответствуюшими образующими. Заметим, что минимальная резольвента единственна с точностью до изоморфизма. 
ПримеР 1.4 .3 (резольвента Кошуля). Пусть $M=\mathbf{k}$. Структура $\mathbf{k}\left[v_{1}, \ldots, v_{m}\right]$-модуля в $\mathbf{k}$ определяется при помоши отображения $\mathbf{k}\left[v_{1}, \ldots, v_{m}\right] \rightarrow \mathbf{k}$, которое переводит все образуюшие $v_{i}$ в 0 . Пусть $\Lambda\left[u_{1}, \ldots, u_{m}\right]$ - внешняя алгебра от $m$ переменных. Превратим тензорное произведение $R=\Lambda\left[u_{1}, \ldots, u_{m}\right] \otimes \mathbf{k}\left[v_{1}, \ldots, v_{m}\right]$ (здесь и далее $\otimes$ обозначает $\left.\otimes_{\mathbf{k}}\right)$ в дифференииальную биградуированную алгебру, полагая

$$
\operatorname{bideg} u_{i}=(-1,2), \quad \operatorname{bideg} v_{i}=(0,2) \text {, }
$$

$$
d u_{i}=v_{i}, \quad d v_{i}=0
$$

и требуя, чтобы $d$ являлось дифференцированием алгебр. Можно показать (см. [56; $\S 7.2])$, что $H^{-i}[R, d]=0$ при $i>0$ и $H^{0}[R, d]=\mathbf{k}$. Так как алгебра $\Lambda\left[u_{1}, \ldots, u_{m}\right] \otimes \mathbf{k}\left[v_{1}, \ldots, v_{m}\right]$ является свободным $\mathbf{k}\left[v_{1}, \ldots, v_{m}\right]$-модулем, она определяет свободную резольвенту модуля $\mathbf{k}$. Эта резольвента назьвается резольвентой Кошуля. Она имеет следуюший вид:

$$
\begin{aligned}
0 \longrightarrow \Lambda^{m}\left[u_{1}, \ldots, u_{m}\right] \otimes \mathbf{k}\left[v_{1}, \ldots, v_{m}\right] \longrightarrow \cdots \\
\quad \ldots \longrightarrow \Lambda^{1}\left[u_{1}, \ldots, u_{m}\right] \otimes \mathbf{k}\left[v_{1}, \ldots, v_{m}\right] \longrightarrow \mathbf{k}\left[v_{1}, \ldots, v_{m}\right] \longrightarrow \mathbf{k} \longrightarrow 0
\end{aligned}
$$

где $\Lambda^{i}\left[u_{1}, \ldots, u_{m}\right]$ - подмодуль в $\Lambda\left[u_{1}, \ldots, u_{m}\right]$, порожденный мономами длины $i$. Таким образом, в обозначениях (14) мы имеем $R^{-i}=\Lambda^{i}\left[u_{1}, \ldots, u_{m}\right] \otimes \mathbf{k}\left[v_{1}, \ldots, v_{m}\right]$.

Пусть $N$ - другой модуль; тогда, применяя функтор $\otimes_{\mathbf{k}\left[v_{1}, \ldots, v_{m}\right]} N$ к (14), мы получаем коцепной комплекс градуированных модулей

$$
0 \longrightarrow R^{-h} \otimes_{\mathbf{k}\left[v_{1}, \ldots, v_{m}\right]} N \longrightarrow \cdots \longrightarrow R^{0} \otimes_{\mathbf{k}\left[v_{1}, \ldots, v_{m}\right]} N \longrightarrow 0
$$

и биградуированный дифференциальный модуль $[R \otimes N, d]$. Модуль $(-i)$-х когомологий последнего коцепного комплекса обозначается $\operatorname{Tor}_{\mathbf{k}\left[v_{1}, \ldots, v_{m}\right]}^{-i}(M, N)$, т.е.

$$
\begin{aligned}
\operatorname{Tor}_{\mathbf{k}\left[v_{1}, \ldots, v_{m}\right]}^{-i}(M, N): & H^{-i}\left[R \otimes_{\mathbf{k}\left[v_{1}, \ldots, v_{m}\right]} N, d\right] \\
& =\frac{\operatorname{Ker}\left[d: R^{-i} \otimes_{\mathbf{k}\left[v_{1}, \ldots, v_{m}\right]} N \rightarrow R^{-i+1} \otimes_{\mathbf{k}\left[v_{1}, \ldots, v_{m}\right]} N\right]}{d\left(R^{-i-1} \otimes_{\mathbf{k}\left[v_{1}, \ldots, v_{m}\right]} N\right)} .
\end{aligned}
$$

Так как $R^{-i}$ и $N$ являются градуированньми модулями, мы имеем

$$
\operatorname{Tor}_{\mathbf{k}\left[v_{1}, \ldots, v_{m}\right]}^{-i}(M, N)=\bigoplus_{j} \operatorname{Tor}_{\mathbf{k}\left[v_{1}, \ldots, v_{m}\right]}^{-i, j}(M, N)
$$

где

$$
\operatorname{Tor}_{\mathbf{k}\left[v_{1}, \ldots, v_{m}\right]}^{-i, j}(M, N)=\frac{\operatorname{Ker}\left[d:\left(R^{-i} \otimes_{\mathbf{k}\left[v_{1}, \ldots, v_{m}\right]} N\right)^{j} \rightarrow\left(R^{-i+1} \otimes_{\mathbf{k}\left[v_{1}, \ldots, v_{m}\right]} N\right)^{j}\right]}{d\left(R^{-i-1} \otimes_{\mathbf{k}\left[v_{1}, \ldots, v_{m}\right]} N\right)^{j}} .
$$

Таким образом, мы имеем биградуированньй $\mathbf{k}\left[v_{1}, \ldots, v_{m}\right]$-модуль

$$
\operatorname{Tor}_{\mathbf{k}\left[v_{1}, \ldots, v_{m}\right]}(M, N)=\bigoplus_{i, j} \operatorname{Tor}_{\mathbf{k}\left[v_{1}, \ldots, v_{m}\right]}^{-i, j}(M, N)
$$

Хорошо известны следуюшие основные свойства модулей $\operatorname{Tor}_{\mathbf{k}\left[v_{1}, \ldots, v_{m}\right]}^{-i}(M, N)($ см., например, [56]). 
ПРЕДЛОЖЕНИЕ 1.4.4. (а) Модуль $\operatorname{Tor}_{\mathbf{k}\left[v_{1}, \ldots, v_{m}\right]}^{-i}(M, N)$ не зависит, с точностъю до изоморфизма, от вибора резольвентьи (14).

(б) $\operatorname{Tor}_{\mathbf{k}\left[v_{1}, \ldots, v_{m}\right]}^{-i}(\cdot, N)$ и $\operatorname{Tor}_{\mathbf{k}\left[v_{1}, \ldots, v_{m}\right]}^{-i}(M, \cdot)$ являются ковариантными функторами.

(в) $\operatorname{Tor}_{\mathbf{k}\left[v_{1}, \ldots, v_{m}\right]}^{0}(M, N) \cong M \otimes_{\mathbf{k}\left[v_{1}, \ldots, v_{m}\right]} N$.

(г) $\operatorname{Tor}_{\mathbf{k}\left[v_{1}, \ldots, v_{m}\right]}^{-i}(M, N) \cong \operatorname{Tor}_{\mathbf{k}\left[v_{1}, \ldots, v_{m}\right]}^{-i}(N, M)$.

Можно также определить $A$-модули $\operatorname{Tor}_{A}(M, N)$ для любой конечно-порожденной градуированной коммутативной алгебры $A$ и (конечнопорожденных градуированных) $A$-модулей $M, N$. Хотя $A$-свободная резольвента (14) модуля $M$ существует не всегда, тем не менее, имеется проективная резольвента модуля $M$, которая позволяет определить $\operatorname{Tor}_{A}(M, N)$ таким же образом, как это делалось выше. Заметим, что проективные модули над алгеброй многочленов являются свободными. Это утверждение было известно как проблема Серра и доказано Квилленом и Суслиным. Для градуированных модулей, однако, этот факт доказывается достаточно просто. В настоящем обзоре модули $\operatorname{Tor}_{A}(M, N)$ для алгебры $A$, отличной от кольца многочленов, встречаются лишш в $\S \S 4.1$ и 5.3 .

Пусть теперь $K^{n-1}$ - симплициальньй комплекс с $m$ вершинами, $M=\mathbf{k}(K)$ и $N=\mathbf{k}$. Так как $\operatorname{deg} v_{i}=2$, мы имеем

$$
\operatorname{Tor}_{\mathbf{k}\left[v_{1}, \ldots, v_{m}\right]}(\mathbf{k}(K), \mathbf{k})=\bigoplus_{i, j=0}^{m} \operatorname{Tor}_{\mathbf{k}\left[v_{1}, \ldots, v_{m}\right]}^{-i, 2 j}(\mathbf{k}(K), \mathbf{k})
$$

(т.е. ненулевые элементы модуля $\operatorname{Tor}_{\mathbf{k}\left[v_{1}, \ldots, v_{m}\right]}(\mathbf{k}(K), \mathbf{k})$ всегда имеют четную вторую градуировку). Определим биградуированные числа Бетти кольца $\mathbf{k}(K)$ как

$$
\beta^{-i, 2 j}(\mathbf{k}(K)):=\operatorname{dim}_{\mathbf{k}} \operatorname{Tor}_{\mathbf{k}\left[v_{1}, \ldots, v_{m}\right]}^{-i, 2 j}(\mathbf{k}(K), \mathbf{k}), \quad 0 \leqslant i, j \leqslant m .
$$

Предположим, что (14) является минимальной свободной резольвентой для $M=\mathbf{k}(K)$. Тогда $R^{0} \cong \mathbf{k}\left[v_{1}, \ldots, v_{m}\right]$ является свободньм $\mathbf{k}\left[v_{1}, \ldots, v_{m}\right]$-модулем с одной образующей степени 0 . Базис модуля $R^{-1}$ (минимальный набор образующих для $\left.\operatorname{Ker}\left[\mathbf{k}\left[v_{1}, \ldots, v_{m}\right] \rightarrow \mathbf{k}(K)\right]\right)$ состоит из элементов $v_{i_{1}, \ldots, i_{k}}, \operatorname{deg} v_{i_{1}, \ldots, i_{k}}=2 k$, таких, что $\left\{i_{1}, \ldots, i_{k}\right\}$ - недостающая грань комплекса $K$. Отображение $d: R^{-1} \rightarrow R^{0}$ переводит $v_{i_{1}, \ldots, i_{k}}$ в $v_{i_{1}} \cdots v_{i_{k}}$. Так, все отображения $d$ в (14) являются минимальнњми, все дифференциалы коцепного комплекса

$$
0 \longrightarrow R^{-h} \otimes_{\mathbf{k}\left[v_{1}, \ldots, v_{m}\right]} \mathbf{k} \longrightarrow \cdots \longrightarrow R^{0} \otimes_{\mathbf{k}\left[v_{1}, \ldots, v_{m}\right]} \mathbf{k} \longrightarrow 0
$$

являются тривиальными. Таким образом, для минимальной резольвенты кольца $\mathbf{k}(K)$ имеет место

$$
\begin{gathered}
\operatorname{Tor}_{\mathbf{k}\left[v_{1}, \ldots, v_{m}\right]}^{-i}(\mathbf{k}(K), \mathbf{k}) \cong R^{-i} \otimes_{\mathbf{k}\left[v_{1}, \ldots, v_{m}\right]} \mathbf{k} \\
\beta^{-i, 2 j}(\mathbf{k}(K))=\operatorname{rank} R^{-i, 2 j}\left(=\operatorname{dim}_{\mathbf{k}\left[v_{1}, \ldots, v_{m}\right]} R^{-i, 2 j}\right) .
\end{gathered}
$$

Числа Бетти $\beta^{-i, 2 j}(\mathbf{k}(K))$ являются важными комбинаторными инвариантами симплишиального комплекса $K$. Ряд результатов об этих числах получен в [82]. Следующий важньй результат, полученный комбинаторньми методами, сводит вычисление чисел $\beta^{-i, 2 j}(\mathbf{k}(K))$ к вычислению гомологий подкомплексов комплекса $K$. 
ТеОрема 1.4.5 (Хохстер [46]). Ряд Пуанкаре модуля $\operatorname{Tor}_{\mathbf{k}\left[v_{1}, \ldots, v_{m}\right]}^{-i}(\mathbf{k}(K), \mathbf{k})$ может быть вычислен при помощи следующей формуль:

$$
\sum_{j} \beta^{-i, 2 j}(\mathbf{k}(K)) t^{2 j}=\sum_{I \subset[m]}\left(\operatorname{dim}_{\mathbf{k}} \widetilde{H}_{\# I-i-1}\left(K_{I}\right)\right) t^{2(\# I)},
$$

әде $K_{I}-$ подкомплекс в $K$, состоящий из симплексов с веричнами в $I$.

Заметим, что вычисления с использованием этой теоремы становятся очень громоздкими даже для малых $K$. В главе 4 мы показываем, что числа $\beta^{-i, 2 j}(\mathbf{k}(K))$ равны биградуированным числам Бетти момент-угол комплекса $\mathscr{Z}_{K}$, ассоциированного с симплициальным комплексом $K$. Это дает альтернативньй, топологический, способ вьгисления чисел $\beta^{-i, 2 j}(\mathbf{k}(K))$.

Теперь рассмотрим резольвенту Кошуля (пример 1.4.3).

ЛЕмма 1.4.6. Для любого модуля $M$ имеем

$$
\operatorname{Tor}_{\mathbf{k}\left[v_{1}, \ldots, v_{m}\right]}(M, \mathbf{k}) \cong H\left[\Lambda\left[u_{1}, \ldots, u_{m}\right] \otimes M, d\right],
$$

где $H\left[\Lambda\left[u_{1}, \ldots, u_{m}\right] \otimes M, d\right]-$ когомологии биградуированного дифференииального модуля $\Lambda\left[u_{1}, \ldots, u_{m}\right] \otimes M$, а d определяется, как в (16).

ДокАЗАТЕльство. Используя в определении модуля $\operatorname{Tor}_{\mathbf{k}\left[v_{1}, \ldots, v_{m}\right]}(\mathbf{k}, M)$ резольвенту Кошуля $\left[\Lambda\left[u_{1}, \ldots, u_{m}\right] \otimes \mathbf{k}\left[v_{1}, \ldots, v_{m}\right], d\right]$, мы находим

$$
\begin{aligned}
\operatorname{Tor}_{\mathbf{k}\left[v_{1}, \ldots, v_{m}\right]}(M, \mathbf{k}) & \cong \operatorname{Tor}_{\mathbf{k}\left[v_{1}, \ldots, v_{m}\right]}(\mathbf{k}, M) \\
& =H\left[\Lambda\left[u_{1}, \ldots, u_{m}\right] \otimes \mathbf{k}\left[v_{1}, \ldots, v_{m}\right] \otimes_{\mathbf{k}\left[v_{1}, \ldots, v_{m}\right]} M\right] \\
& \cong H\left[\Lambda\left[u_{1}, \ldots, u_{m}\right] \otimes M\right] .
\end{aligned}
$$

Предположим теперь, что $\mathbf{k}\left[v_{1}, \ldots, v_{m}\right]$-модуль $M$ является $\mathbf{k}$-алгеброй. Тогда когомологии алгебры $\left[\Lambda\left[u_{1}, \ldots, u_{m}\right] \otimes M, d\right]$ также являются алгеброй. Лемма 1.4 .6 позволяет снабдить модуль $\operatorname{Tor}_{\mathbf{k}\left[v_{1}, \ldots, v_{m}\right]}(M, \mathbf{k})$ канонической структурой конечномерной биградуированной $\mathbf{k}$-алгебры. Мы будем называть эту алгебру Tor-алгеброй алгебры $M$. Tor-алгеброй симплиииального комплекса $K$ называется Tor-алгебра его кольца граней $\mathbf{k}(K)$.

ЗАмечАниЕ. В общем случае, когда $N \neq \mathbf{k}$, модули $\operatorname{Tor}_{\mathbf{k}\left[v_{1}, \ldots, v_{m}\right]}(M, N)$ не имеют канонической структуры алгебры, даже если и $M$ и $N$ являются алгебрами.

Конструкция 1.4.7 (мультиградуированная структура в Tor-алгебре). Введем в кольце многочленов $\mathbf{k}\left[v_{1}, \ldots, v_{m}\right]$ мультиградуировку (точнее, $\mathbb{N}^{m}$-градуировку), полагая mdeg $v_{i}=(0, \ldots, 0,2,0, \ldots, 0)$, где 2 стоит на $i$-м месте. При этом моном $v_{1}^{i_{1}} \cdots v_{m}^{i_{m}}$ приобретает мультистепень $\left(2 i_{1}, \ldots, 2 i_{m}\right)$. Пусть алгебра $M$ представляет собой фактор-кольцо кольца многочленов по мономиальному идеалу. Тогда введенная выше мультиградуировка индуцирует мультиградуировку в алгебре $M$, а также во всех членах резольвенты (14). Мы можем предполагать, что дифференциалы в резольвенте сохраняют мультистепени. Следовательно, модули $\operatorname{Tor}_{\mathbf{k}\left[v_{1}, \ldots, v_{m}\right]}(M, N)$ для таких алгебр имеют каноническую $\mathbb{N} \oplus \mathbb{N}^{m}$-градуировку, т.е.

$$
\operatorname{Tor}_{\mathbf{k}\left[v_{1}, \ldots, v_{m}\right]}(M, \mathbf{k})=\bigoplus_{i \geqslant 0, \boldsymbol{j} \in \mathbb{N}^{m}} \operatorname{Tor}_{\mathbf{k}\left[v_{1}, \ldots, v_{m}\right]}^{-i, \boldsymbol{j}}(M, \mathbf{k}) .
$$

Таким образом, Тог-алгебра комплекса $K$ приобретает $\mathbb{N} \oplus \mathbb{N}^{m}$-градуировку. 
ЗАмЕчАниЕ. В соответствии с нашим соглашением первая градуировка в Tor-алгебре всегда неположительна (напомним, что мы занумеровали члены резольвенты Кошуля неположительными числами). В этих обозначениях комплекс Кошуля $\left[M \otimes \Lambda\left[u_{1}, \ldots, u_{m}\right], d\right]$ становится коцепныцм комплексом, и $\operatorname{Tor}_{\mathbf{k}\left[v_{1}, \ldots, v_{m}\right]}(M, \mathbf{k})$ есть его когомологии, а не гомологии, как обычно принято. Это стандартный прием, используемый для применения спектральной последовательности Эйленберга-Мура, см. § 4.1. Это также объясняет, почему мы пишем $\operatorname{Tor}_{\mathbf{k}\left[v_{1}, \ldots, v_{m}\right]}^{*}(M, \mathbf{k})$ вместо обычного $\operatorname{Tor}_{*, *}^{\mathbf{k}\left[v_{1}, \ldots, v_{m}\right]}(M, \mathbf{k})$.

Верхняя граница hd $M \leqslant m$ из теоремы Гильберта о сизигиях может быть заменена следуюшим более точным результатом.

Tеорема 1.4.8 (Ауслендер, Буксбаум). hd $M=m-\operatorname{depth} M$.

Далее мы предполагаем, что алгебра $M$ порождена элементами степени два и структура $\mathbf{k}\left[v_{1}, \ldots, v_{m}\right]$-модуля на $M$ задается при помоши некоторого эпиморфизма $p: \mathbf{k}\left[v_{1}, \ldots, v_{m}\right] \rightarrow M$ (это всегда вьполнено, если $M=\mathbf{k}(K)$ для некоторого симплициального комплекса $K$ ). Пусть $\theta_{1}, \ldots, \theta_{k}$ - регулярная последовательность элементов степени два в $M$. Положим $\mathscr{J}:=\left(\theta_{1}, \ldots, \theta_{k}\right) \subset M$ (идеал, порожденный элементами $\left.\theta_{1}, \ldots, \theta_{k}\right)$. Выберем элементы $t_{i} \in \mathbf{k}\left[v_{1}, \ldots, v_{m}\right]$ степени два такие, что $p\left(t_{i}\right)=\theta_{i}, i=1, \ldots, k$. Идеал в $\mathbf{k}\left[v_{1}, \ldots, v_{m}\right]$, порожденный элементами $t_{1}, \ldots, t_{k}$, также будет обозначаться $\mathscr{J}$. Тогда $\mathbf{k}\left[v_{1}, \ldots, v_{m}\right] / \mathscr{J} \cong \mathbf{k}\left[w_{1}, \ldots, w_{m-k}\right]$. При этих предположениях имеет место следуюшая лемма о редукции.

Лемма 1.4.9. Имеет место следующий изоморфизм алгебр:

$$
\operatorname{Tor}_{\mathbf{k}\left[v_{1}, \ldots, v_{m}\right]}(M, \mathbf{k})=\operatorname{Tor}_{\mathbf{k}\left[v_{1}, \ldots, v_{m}\right] / \mathscr{J}}(M / \mathscr{J}, \mathbf{k}) .
$$

Для доказательства леммы нам понадобится следующий факт из гомологической алгебры.

Tеорема 1.4 .10 [28; с. 349]. Пусть $\Lambda$ - алгебра, Г - ее подалгебра и $\Omega=\Lambda / \Gamma-$ фактор-алгебра. Предположим, что $\Lambda$ является свободным Г-модулем и даны некоторые $\Omega$-модуль А и $\Lambda$-модуль $C$. Тогда существует спектральная последовательность $\left\{E_{r}, d_{r}\right\}$ такая, что

$$
E_{r} \Rightarrow \operatorname{Tor}_{\Lambda}(A, C), \quad E_{2}=\operatorname{Tor}_{\Omega}\left(A, \operatorname{Tor}_{\Gamma}(C, \mathbf{k})\right) .
$$

ДОКАЗАТЕЛЬСТвО ЛЕМмы 1.4.9. Положим $\Lambda=\mathbf{k}\left[v_{1}, \ldots, v_{m}\right], \Gamma=\mathbf{k}\left[t_{1}, \ldots, t_{k}\right]$, $A=\mathbf{k}, C=M$. Тогда $\Lambda$ есть свободньй Г-модуль и $\Omega=\Lambda / \Gamma=\mathbf{k}\left[v_{1}, \ldots, v_{m}\right] / \mathscr{J}$. Таким образом, согласно теореме 1.4.10 мы имеем спектральную последовательность

$$
E_{r} \Rightarrow \operatorname{Tor}_{\mathbf{k}\left[v_{1}, \ldots, v_{m}\right]}(M, \mathbf{k}), \quad E_{2}=\operatorname{Tor}_{\Omega}\left(\operatorname{Tor}_{\Gamma}(M, \mathbf{k}), \mathbf{k}\right) .
$$

Так как $\theta_{1}, \ldots, \theta_{k}$ - регулярная последовательность, $M$ является свободным $\Gamma$-модулем. Следовательно,

$$
\operatorname{Tor}_{\Gamma}(M, \mathbf{k})=M \otimes_{\Gamma} \mathbf{k}=M / \mathscr{J} \text { и } \operatorname{Tor}_{\Gamma}^{q}(M, \mathbf{k})=0 \text { при } q \neq 0 .
$$


Отсюда вытекает, что $E_{2}^{p, q}=0$ при $q \neq 0$. Таким образом, спектральная последовательность вырождается в члене $E_{2}$, и

$$
\operatorname{Tor}_{\mathbf{k}\left[v_{1}, \ldots, v_{m}\right]}(M, \mathbf{k})=\operatorname{Tor}_{\Omega}\left(\operatorname{Tor}_{\Gamma}(M, \mathbf{k}), \mathbf{k}\right)=\operatorname{Tor}_{\mathbf{k}\left[v_{1}, \ldots, v_{m}\right] / \mathscr{J}}(M / \mathscr{J}, \mathbf{k}),
$$

что завершает доказательство.

Из леммы 1.4.9 вытекает, что если $M$ является алгеброй Коэна-Маколея размерности Крулля $n$, то $\operatorname{depth} M=n$, hd $M=m-n$ и $\operatorname{Tor}_{\mathbf{k}\left[v_{1}, \ldots, v_{m}\right]}^{-i}(M, \mathbf{k})=0$ при $i>m-n$.

ОПРЕДЕЛЕНИЕ 1.4.11. Пусть $\mathbf{k}\left[v_{1}, \ldots, v_{m}\right]$-модуль $M$ является алгеброй КоэнаМаколея размерности Крулля $n$. Тогда $M$ называется горенштейновой алгеброй, если $\operatorname{Tor}_{\mathbf{k}\left[v_{1}, \ldots, v_{m}\right]}^{-(m-n)}(M, \mathbf{k}) \cong \mathbf{k}$.

Следуя Стенли [82], мы называем симплициальньй комплекс $K$ горенштейновым, если $\mathbf{k}(K)$ является горенштейновой алгеброй. Далее, $K$ называется горенштейно$\boldsymbol{s} \boldsymbol{b}$ м $^{*}$, если $\mathbf{k}(K)$ является горенштейновой алгеброй и $K=\operatorname{core} K$ (cм. $\left.\S 1.2\right)$. Следующая теорема дает комбинаторное описание горенштейновых * симплициальных комплексов.

ТЕОРема 1.4.12 [82; §II.5]. Симплициальный комплекс $K$ является горенштейновьм.м над $\mathbf{k}$ тогда и только тогда, когда для любого симплекса $I \in K$ (включая $I=\varnothing)$ подкомплекс $\operatorname{link} I$ имеет гомологии как у сферы размерности $\operatorname{dim}(\operatorname{link} I)$.

В частности, симплициальные сферы и симплициальные гомологические сферы (симплициальные многообразия, имеющие гомологии как у сферы) являются горенштейновыми* комплексами. Заметим, однако, что горенштейнов* комплекс не обязательно является симплициальным многообразием (линки вершин не обязательно односвязны, сравните с теоремой 1.2.11).

Теорема 1.4.13 [82; §II.5]. Пусть $K^{n-1}$ - горенштейнов* комплекс. Тогда для рядов Пуанкаре модулей $\operatorname{Tor}_{\mathbf{k}\left[v_{1}, \ldots, v_{m}\right]}^{-i}(\mathbf{k}(K), \mathbf{k}), 0 \leqslant i \leqslant m-n$, uмеют место тождества

$$
F\left(\operatorname{Tor}_{\mathbf{k}\left[v_{1}, \ldots, v_{m}\right]}^{-i}(\mathbf{k}(K), \mathbf{k}) ; t\right)=t^{2 m} F\left(\operatorname{Tor}_{\mathbf{k}\left[v_{1}, \ldots, v_{m}\right]}^{-(m-n)+i}(\mathbf{k}(K), \mathbf{k}) ; \frac{1}{t}\right)
$$

СЛЕДСТВИЕ 1.4.14. Если $K^{n-1}$ - горенштейнов * комплекс, то

$$
F(\mathbf{k}(K), t)=(-1)^{n} F\left(\mathbf{k}(K), \frac{1}{t}\right)
$$

ДокАЗАТЕЛЬСтво. Применим теорему $1.4 .1 \mathrm{k}$ минимальной резольвенте алгебры $\mathbf{k}(K)$. Как вытекает из (18), слагаемые в правой части формулы (15) суть в точности $F\left(\operatorname{Tor}_{\mathbf{k}\left[v_{1}, \ldots, v_{m}\right]}^{-i}(\mathbf{k}(K), \mathbf{k}) ; t\right), i=0, \ldots, m-n$. Таким образом,

$$
F(\mathbf{k}(K) ; t)=\left(1-t^{2}\right)^{-m} \sum_{i=0}^{m-n}(-1)^{i} F\left(\operatorname{Tor}_{\mathbf{k}\left[v_{1}, \ldots, v_{m}\right]}^{-i}(\mathbf{k}(K), \mathbf{k}) ; t\right)
$$


Используя теорему 1.4.13, мы получаем

$$
\begin{aligned}
F(\mathbf{k}(K) ; t) & =\left(1-t^{2}\right)^{-m} \sum_{i=0}^{m-n}(-1)^{i} t^{2 m} F\left(\operatorname{Tor}_{\mathbf{k}\left[v_{1}, \ldots, v_{m}\right]}^{-(m-n)+i}(\mathbf{k}(K), \mathbf{k}) ; \frac{1}{t}\right) \\
& =\left(1-\left(\frac{1}{t}\right)^{2}\right)^{-m}(-1)^{m} \sum_{j=0}^{m-n}(-1)^{m-n-j} F\left(\operatorname{Tor}_{\mathbf{k}\left[v_{1}, \ldots, v_{m}\right]}^{-j}(\mathbf{k}(K), \mathbf{k}) ; \frac{1}{t}\right) \\
& =(-1)^{n} F\left(\mathbf{k}(K) ; \frac{1}{t}\right) .
\end{aligned}
$$

СлеДСТвИЕ 1.4.15. Соотношения Дена-Соммервилля $h_{i}=h_{n-i}, 0 \leqslant i \leqslant n$, имеют место для любого горенштейнова комплекса $K^{n-1}$ (в частности, для любой симплищиальной сферь).

ДокАЗАТЕльство. Это вытекает из леммы 1.3.3 и следствия 1.4.14.

Как было отмечено Стенли в [81], горенштейновы* комплексы являются наиболее обшими объектами, пригодными для обобшения $g$-теоремы (как мы отмечали, многогранные сферы, $P L$-сферы, симплициальные сферы и симплициальные гомологические сферы являются частньми случаями горенштейновых ${ }^{*}$ комплексов).

Соотношения Дена-Соммервилля допускают обобшение даже на более широкий класс объектов, чем горенштейновы* комплексы. В работе [52] Кли получил соотношения Дена-Соммервилля в виде (10) в более обшем контексте әйлеровых многообразий. В частности, отсюда вытекает, что уравнения (10) (за исключением $k=0$ ) имеют место для произвольного симплициального многообразия $K$ размерности $n-1$ (в случае $k=0$ уравнение (10) выражает тот факт, что $\chi\left(K^{n-1}\right)=\chi\left(S^{n-1}\right)$ ). Аналоги соотношений (10) были получены Байером и Биллерой в [12] (для әйлеровых частично упорядоченных множеств) и Ченом и Яном [30] (для произвольных полиэдров).

В $\S 4.5$ мы получаем (топологическими методами) соотношения Дена-Соммервилля для симплициальных многообразий в виде:

$$
h_{n-i}-h_{i}=(-1)^{i}\left(\chi\left(K^{n-1}\right)-\chi\left(S^{n-1}\right)\right)\left(\begin{array}{c}
n \\
i
\end{array}\right), \quad i=0,1, \ldots, n,
$$

где $\chi\left(K^{n-1}\right)=f_{0}-f_{1}+\cdots+(-1)^{n-1} f_{n-1}=1+(-1)^{n-1} h_{n}-$ эйлерова характеристика комплекса $K^{n-1}$, а $\chi\left(S^{n-1}\right)=1+(-1)^{n-1}$ - эйлерова характеристика сферы. Заметим, что если $K$ является симплициальной сферой или имеет нечетную размерность, то полученные соотношения принимают классический вид $h_{n-i}=h_{i}$.

§1.5. Кубические комплексы и кубические отображения. Определим $q$ мерньй комбинаторно-геометрический куб как многогранник, комбинаторно эквивалентный единичному $q$-кубу (3). В этом параграфе под кубами мы подразумеваем комбинаторно-геометрические кубы.

ОПРЕДЕЛЕНИЕ 1.5.1. Кубический комплекс - это подмножество $\mathscr{C} \subset \mathbb{R}^{n}$, представленное в виде объединения кубов любых размерностей таким образом, что пересечение любых двух кубов является гранью каждого из них. 
ЗАмЕчАниЕ. Предыдущее определение кубического комплекса аналогично определению 1.2.2 геометрического симплициального комплекса. Можно также определить абстрактный кубический комплекс, однако это определение более тонко (и нам оно не потребуется).

Гранью кубического комплекса $\mathscr{C}$ назьвается любая грань куба из $\mathscr{C}$. Размерность комплекса $\mathscr{C}$ есть максимальная размерность его граней. Для кубического комплекса $\mathscr{C}$ естественным образом вводится понятие $f$-вектора $\left(f_{i}\right.$ есть число $i$-граней). Некоторые проблемы, связанные с $f$-векторами кубических комплексов, приведены в [83].

Очевидно, стандартный куб $I^{q}$ (вместе со всеми своими гранями) является $q$-мерным кубическим комплексом, которьй мы также будем обозначать $I^{q}$. Любая грань $I^{q}$ имеет вид

$$
C_{I \subset J}=\left\{\left(y_{1}, \ldots, y_{q}\right) \in I^{q}: y_{i}=0 \text { при } i \in I, y_{i}=1 \text { при } i \notin J\right\},
$$

где $I \subset J$-два (возможно пустых) подмножества в $[q]$. Мы также введем обозначение $C_{J}:=C_{\varnothing \subset J}$.

В отличие от симплициальных комплексов (которые всегда являются подкомплексами симплекса), не любой кубический комплекс может быть реализован как подкомплекс в некотором $I^{q}$. Один из примеров кубического комплекса, не вложимого в качестве подкомплекса ни в какой куб $I^{q}$, показан на рис. 1 . Более того, этот комплекс не вложим в стандартную кубическую решетку в $\mathbb{R}^{q}$ (ни для какого $q$ ). Авторы благодарны М. И. Штогрину за этот пример.

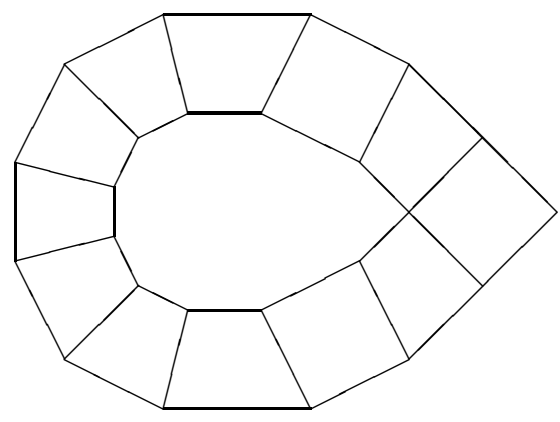

Рис. 1. Кубический комплекс, не вложимый в кубическую решетку

Выделим следующую проблему.

ПроБлЕмА 1.5 .2 (С.П. Новиков). Охарактеризовать $k$-мерные кубические комплексы $\mathscr{C}$ (в особенности те из них, которые являются многообразиями), допускающие

(а) (кубическое) вложение в стандартную кубическую решетку в $\mathbb{R}^{q}$;

(б) отображсение в стандартную кубическую решетку в $\mathbb{R}^{q}$, ограничение которого на каждый $k$-мерный куб отождествляет его с некоторой $k$-гранью решетки.

В случае, когда $\mathscr{C}$ гомеоморфно $S^{2}$, эта проблема решена в работе [37]. Проблема 1.5.2 явилась развитием следуюшей проблемы, приведенной в [37]. 
ПроБЛЕмА 1.5.3 (С.П. Новиков). Пусть задан некоторый двумерный кубический цикл $\alpha$ по модулю 2 в стандартной кубической решетке пространства $\mathbb{R}^{3}$. Описать отображения кубических разбиений двумерных поверхностей на цикл $\alpha$, при которых никакие два различных квадрата не отображаются на один квадрат иикла.

Как сообщил авторам С. П. Новиков, проблема 1.5.3 возникла в ходе его совместных обсуждений с известным физиком А. М. Поляковым трехмерной модели Изинга.

Далее мы вводим специальные кубические комплексы, которые будут играть ключевую роль в нашей теории момент-угол комплексов. Каждьй из этих кубических комплексов допускает каноническое кубическое вложение в стандартный куб. Обратим внимание, что задачи о вложениях в стандартную кубическую решетку и стандартный куб тесно связаны. Например, как показано в [37], если кубическое разбиение двумерной поверхности вкладьвается в стандартную кубическую решетку в $\mathbb{R}^{q}$, то оно также допускает кубическое вложение в $I^{q}$.

Конструкция 1.5.4 (каноническое симплициальное разбиение куба $I^{m}$ ). Пусть $\Delta^{m-1}$ - симплекс на множестве $[m]$, т.е. $\Delta^{m-1}$ есть набор всех подмножеств $[m]$. Поставим в соответствие каждому подмножеству $I=\left\{i_{1}, \ldots, i_{k}\right\} \subset[m]$ вершину $v_{I}:=C_{I \subset I}$ куба $I^{m}$. Таким образом, $v_{I}=\left(\varepsilon_{1}, \ldots, \varepsilon_{m}\right)$, где $\varepsilon_{i}=0$, если $i \in I$, и $\varepsilon_{i}=1$ иначе. Рассматривая подмножество $I$ как некоторую вершину барицентрического подразделения симплекса $\Delta^{m-1}$, мы можем продолжить наше соответствие $I \mapsto v_{I}$ до кусочно-линейного вложения $i_{c}$ полиэдра $\left|\operatorname{bs}\left(\Delta^{m-1}\right)\right|$ в (граничный комплекс куба) $I^{m}$. При этом вложении вершины полиэдра $\left|\Delta^{m-1}\right|$ переходят в вершины $(1, \ldots, 1,0,1, \ldots, 1) \in I^{m}$, в то время как барицентр симплекса $\left|\Delta^{m-1}\right|$ отображается в вершину $(0, \ldots, 0) \in I^{m}$. Образ $i_{c}\left(\left|\mathrm{bs}\left(\Delta^{m-1}\right)\right|\right)$ представляет собой объединение $m$ гиперграней куба $I^{m}$, сходящихся в вершине $(0, \ldots, 0)$. Для любой пары $I \subset J$ непустых вложенных подмножеств множества $[m]$ все симплексы комплекса $\operatorname{bs}\left(\Delta^{m-1}\right)$ вида $I=I_{1} \subset I_{2} \subset \cdots \subset I_{k}=J$ отображаются на одну и ту же грань $C_{I \subset J} \subset I^{m}$ (см. (19)). Отображение $i_{c}:\left|\operatorname{bs}\left(\Delta^{m-1}\right)\right| \rightarrow I^{m}$ можно продолжить на $\left|\operatorname{cone}\left(\mathrm{bs}\left(\Delta^{m-1}\right)\right)\right|$, переводя вершину конуса в $(1, \ldots, 1) \in I^{m}$. Получаемое отображение обозначим cone $\left(i_{c}\right)$. Его образ есть весь куб $I^{m}$. Следовательно, cone $\left(i_{c}\right):\left|\operatorname{cone}\left(\mathrm{bs}\left(\Delta^{m-1}\right)\right)\right| \rightarrow I^{m}$ является $P L$-гомеоморфизмом, линейным на симплексах полиэдра $\left|\operatorname{cone}\left(\operatorname{bs}\left(\Delta^{m-1}\right)\right)\right|$. Это определяет каноническую триангуляцию куба $I^{m}$. Таким образом, каноническая триангуляция куба $I^{m}$ получается при отождествлении его с конусом над барищентрическим подразделением симплекса $\Delta^{m-1}$.

КонСтрукция 1.5.5 (кубическое разбиение простого многогранника). Пусть $P^{n}$ $\subset \mathbb{R}^{n}$ - простой многогранник с $m$ гипергранями $F_{1}^{n-1}, \ldots, F_{m}^{n-1}$. Выберем по точке во внутренности каждой грани многогранника $P^{n}$ (включая вершины и сам многогранник). В результате мы получаем множество $\mathscr{S}$ из $1+f_{0}+f_{1}+\cdots+f_{n-1}$ точек (здесь $\boldsymbol{f}\left(P^{n}\right)=\left(f_{0}, f_{1}, \ldots, f_{n-1}\right)-f$-вектор многогранника $\left.P^{n}\right)$. Для каждой вершины $v \in P^{n}$ определим подмножество $\mathscr{S}_{v} \subset \mathscr{S}$, состоящее из выбранных точек во внутренностях граней, содержащих $v$. Так как $P^{n}$ является простым, число $k$-граней, сходяшихся в $v$, равно $\left(\begin{array}{l}n \\ k\end{array}\right), 0 \leqslant k \leqslant n$. Таким образом, \# $\mathscr{S}_{v}=2^{n}$. Множество $\mathscr{S}_{v}$ яв- 
ляется множеством вершин некоторого $n$-куба, обозначаемого $C_{v}^{n}$. Грани куба $C_{v}^{n}$ описьваются следующим образом. Пусть $G_{1}^{k} \subset G_{2}^{l}$ - две вложенные грани $P^{n}$, содержашие $v$. Тогда имеется в точности $2^{l-k}$ граней $G$ многогранника $P^{n}$ таких, что $G_{1}^{k} \subset G \subset G_{2}^{l}$. Соответствующие $2^{l-k}$ точек из $\mathscr{S}$ образуют множество вершин некоторой $(l-k)$-грани куба $C_{v}^{n}$. Обозначим эту грань $C_{G_{1} \subset G_{2}}^{l-k}$. Любая грань куба $C_{v}^{n}$ имеет вид $C_{G_{1} \subset G_{2}}^{i}$ для некоторых $G_{1}, G_{2}$, содержаших $v$. Пересечение любых двух кубов $C_{v}^{n}, C_{v^{\prime}}^{n}$ является гранью каждого из них. Действительно, пусть $G^{i} \subset P^{n}$ наименьшая грань, содержашая обе вершины $v$ и $v^{\prime}$. Тогда $C_{v}^{n} \cap C_{v^{\prime}}^{n}=C_{G^{i} \subset P^{n}}^{n-i}$ является гранью как $I_{v}^{n}$, так и $I_{v^{\prime}}^{n}$. Таким образом, мы построили кубическое разбиение многогранника $P^{n}$ с $f_{n-1}\left(P^{n}\right)$ кубами размерности $n$. Обозначим этот кубический комплекс $\mathscr{C}\left(P^{n}\right)$.

Имеется вложение комплекса $\mathscr{C}\left(P^{n}\right)$ в $I^{m}$, которое строится следуюшим образом. Каждая $(n-k)$-грань $G^{n-k}$ многогранника $P^{n}$ есть пересечение $k$ гиперграней: $G^{n-k}=F_{i_{1}}^{n-1} \cap \cdots \cap F_{i_{k}}^{n-1}$. Отобразим соответствуюшую точку множества $\mathscr{S}$ в вершину $\left(\varepsilon_{1}, \ldots, \varepsilon_{m}\right) \in I^{m}$, где $\varepsilon_{i}=0$, если $i \in\left\{i_{1}, \ldots, i_{k}\right\}$, и $\varepsilon_{i}=1$ иначе. Это определяет отображение множества вершин $\mathscr{S}$ комплекса $\mathscr{C}\left(P^{n}\right)$ в множество вершин куба $I^{m}$. Используя каноническую триангуляцию куба $I^{m}$ (конструкция 1.5.4), мы можем продолжить это отображение до $P L$-вложения $i_{P}: P^{n} \rightarrow I^{m}$. Для любой вершины $v=F_{i_{1}}^{n-1} \cap \cdots \cap F_{i_{n}}^{n-1} \in P^{n}$ мы имеем

$$
i_{P}\left(C_{v}^{n}\right)=\left\{\left(y_{1}, \ldots, y_{m}\right) \in I^{m}: y_{j}=1 \text { при } j \notin\left\{i_{1}, \ldots, i_{n}\right\}\right\},
$$

т.е. $i_{P}\left(C_{v}^{n}\right)=C_{\left\{i_{1}, \ldots, i_{n}\right\}} \subset I^{m}$ (в обозначениях (19)). Вложение $i_{P}: P^{n} \rightarrow I^{m}$ для $n=2, m=3$ показано на рис. 2 .
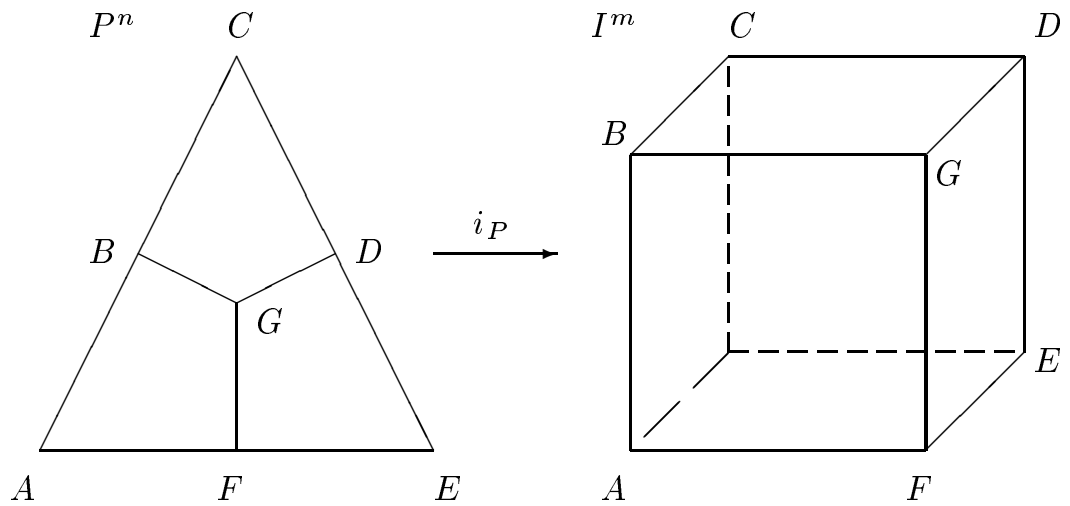

Рис. 2. Вложение $i_{P}: P^{n} \rightarrow I^{m}$ для $n=2, m=3$

Сведем факты из предыдущей конструкции в следуюшем утверждении.

Теорема 1.5.6. Простой многогранник $P^{n}$ с $m$ гипергранями можно разбить на кубъ $C_{v}^{n}$, по одному на каждую вериину $v \in P^{n}$. Получаемый кубический комплекс $\mathscr{C}\left(P^{n}\right)$ канонически вкладывается в граничу куба $I^{m}$, как описано в (20). 
Лемма 1.5.7. Число k-граней кубического комплекса $\mathscr{C}\left(P^{n}\right)$ есть

$$
\begin{aligned}
f_{k}\left(\mathscr{C}\left(P^{n}\right)\right) & =\sum_{i=0}^{n-k}\left(\begin{array}{c}
n-i \\
k
\end{array}\right) f_{n-i-1}\left(P^{n}\right) \\
& =\left(\begin{array}{c}
n \\
k
\end{array}\right) f_{n-1}\left(P^{n}\right)+\left(\begin{array}{c}
n-1 \\
k
\end{array}\right) f_{n-2}\left(P^{n}\right)+\cdots+f_{k-1}\left(P^{n}\right), \quad k=0, \ldots, n .
\end{aligned}
$$

ДокАЗАТЕльство. Это вытекает из того факта, что $k$-грани комплекса $\mathscr{C}\left(P^{n}\right)$ находятся во взаимно однозначном соответствии с парами $G_{1}^{i} \subset G_{2}^{i+k}$ вложенных граней многогранника $P^{n}$.

Конструкция 1.5.8. Пусть $K^{n-1}$ - симплициальный комплекс на множестве $[m]$. Тогда $K$ естественным образом является подкомплексом симплекса $\Delta^{m-1}$ и $\mathrm{bs}(K)$ является подкомплексом в $\operatorname{bs}\left(\Delta^{m-1}\right)$. Как вытекает из конструкции 1.5.4, имеется $P L$-вложение $\left.i_{c}\right|_{\mathrm{bs}(K)}:|\operatorname{bs}(K)| \rightarrow I^{m}$. Образ $i_{c}(|\operatorname{bs}(K)|)$ представляет собой $(n-1)$ мерньй кубический подкомплекс в $I^{m}$, которьй мы обозначаем $\operatorname{cub}(K)$. Тогда

$$
\operatorname{cub}(K)=\bigcup_{\varnothing \neq I \subset J \in K} C_{I \subset J} \subset I^{m},
$$

т.е. $\operatorname{cub}(K)$ есть объединение граней $C_{I \subset J} \subset I^{m}$ по всем парам $I \subset J$ непустых симплексов комплекса $K$.

Конструкция 1.5.9. Поскольку комплекс cone $(\mathrm{bs}(K))$ является подкомплексом в cone $\left(\operatorname{bs}\left(\Delta^{m-1}\right)\right)$, конструкция 1.5.4 также определяет $P L$-вложение

$$
\left.\operatorname{cone}\left(i_{c}\right)\right|_{\text {cone }(\operatorname{bs}(K))}:|\operatorname{cone}(\operatorname{bs}(K))| \rightarrow I^{m} \text {. }
$$

Образ этого вложения представляет собой $n$-мерный кубический подкомплекс в $I^{m}$, обозначаемьй сс $(K)$. Тогда легко видеть, что

$$
\operatorname{cc}(K)=\bigcup_{J \in K} C_{I \subset J} \subset I^{m} .
$$

Так как $C_{I \subset J} \subset C_{\varnothing \subset J}=C_{J}$, мы также можем написать $\operatorname{cc}(K)=\bigcup_{J \in K} C_{J}$.

Следуюшее утверждение сводит вместе результаты двух предыдуших конструкций.

ТЕорема 1.5.10. Для любого симплициального комплекса $K$ на множестве $[\mathrm{m}]$ имеется $P L$-вложсение полиэдра $|K|$ в куб $I^{m}$, линейное на симплексах из $\mathrm{bs}(K)$. Образом этого вложения является кубический подкомплекс (21). Кроме того, имеется $P L$-вложение полиэдра $|\operatorname{cone}(K)|$ в $I^{m}$, линейное на симплексах из cone $(\mathrm{bs}(K))$. Образом этого вложения является кубический подкомплекс (22).

Как и для симплициальных комплексов, кубический комплекс ' $\mathfrak{C}^{\prime}$ называется $к у б и-$ ческим подразделением кубического комплекса $\mathscr{C}$, если каждьй куб из $\mathscr{C}$ есть объединение конечного числа кубов из $\mathscr{C}^{\prime}$. 
ПРЕДЛОЖЕНИЕ 1.5.11. Для каждого кубического комплекса $\mathscr{C}$ существует кубическое подразделение $\mathscr{C}^{\prime}$, которое мохет быть реализовано как подкомплекс в некотором кубе $I^{q}$.

ДокАЗАТЕльство. Разбивая каждый куб комплекса $\mathscr{C}$, как описано в конструкции 1.5.4, мы получаем симплициальный комплекс, скажем, $K_{\mathscr{C}}$. Далее, применяя конструкцию 1.5 .8 к $K_{\mathscr{C}}$, мы получаем кубический комплекс, которьй подразбивает $\left|K_{\mathscr{C}}\right| \cong \mathscr{C}$ и вкладывается в некоторый куб $I^{q}$ (см. теорему 1.5.10) как подкомплекс $\operatorname{cub}\left(K_{\mathscr{C}}\right)$.

ПримеР 1.5.12. На рис. 3 (a) показан кубический комплекс $\operatorname{cub}(K)$ в случае, ког да $K$ является несвязным объединением 3 вершин $(K=: \cdot)$. На рис. 3 (б) показан тот же кубический комплекс в случае, когда $K$ является границей 2 -симплекса $\left(K=\partial \Delta^{2}\right)$. Кубический комплекс $c c(K)$ в обоих случаях показан на рис. 4 (а) и (б).

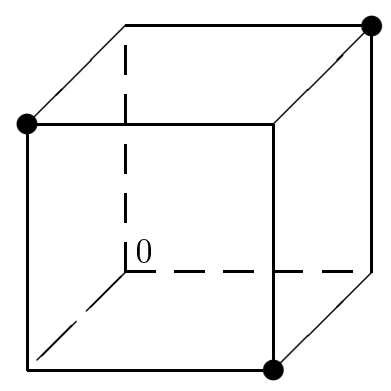

(a) $K=:$ :

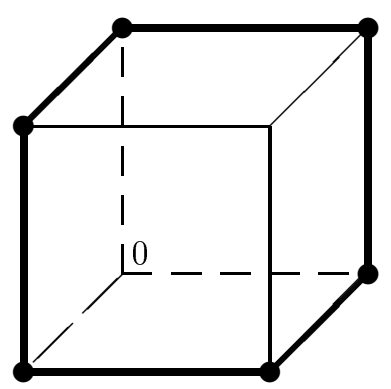

(б) $K=\partial \Delta^{2}$

Рис. 3. Кубический комплекс $\operatorname{cub}(K)$

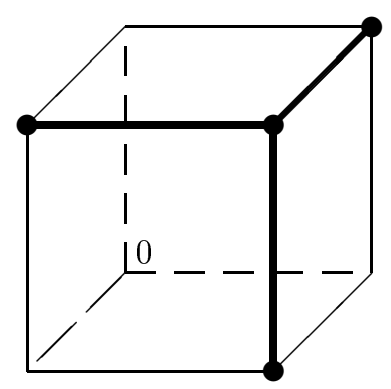

(a) $K=$ :

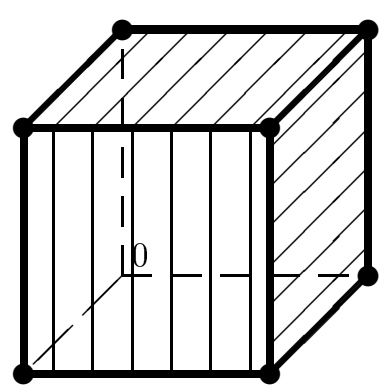

(б) $K=\partial \Delta^{2}$

Рис. 4. Кубический комплекс $\operatorname{cc}(K)$

ЗАмЕчАниЕ. Как топологическое пространство $\operatorname{cub}(K)$ гомеоморфен $|K|$, в то время как cc $(K)$ гомеоморфен $|\operatorname{cone}(K)|$. Для симплициального комплекса cone $(K)$ мы можем построить кубический комплекс $\operatorname{cub}(\operatorname{cone}(K))$, который также гомеоморфен $\mid$ cone $(K) \mid$. Однако, как кубические комплексы сс $(K)$ и cub $(\operatorname{cone}(K))$ отличаются (так как cone $(\operatorname{bs}(K))$ и $\operatorname{bs}(\operatorname{cone}(K))$ - разные симплициальные комплексы). 
Пусть $P$ - простой $n$-многогранник и $K_{P}$ - соответствующая симплициальная $(n-1)$-сфера (граница полярного симплициального многогранника $\left.P^{*}\right)$. Тогда $\operatorname{cc}\left(K_{P}\right)$ совпадает с кубическим комплексом $\mathscr{C}(P)$ из конструкции 1.5.5 (точнее, $\left.\operatorname{cc}\left(K_{P}\right)=i_{P}(\mathscr{C}(P))\right)$. Таким образом, конструкция 1.5 .5 является частным случаем конструкции 1.5.9.

ЗАмЕчАниЕ. Некоторые версии приведенных выше наших конструкций уже появлялись в литературе. Вариант конструкции 1.5.9 можно найти в [33; с. 434] (там он использовался для изучения некоторых действий тора; мы вернемся к этому в следуюшей главе). Версия нашего подкомплекса $\operatorname{cub}(K) \subset I^{m}$ появлялась в [76] в связи с проблемой 1.5.2.

\section{Глава 2. Торические и квазиторические многообразия}

§ 2.1. Алгебраические торические многообразия. Торические многообразия возникли в алгебраической геометрии в начале 1970-х годов в связи с задачей компактификации орбиты действия алгебраического тора (см. ниже). Очень быстро геометрия торических многообразий превратилась в один из самых красивых разделов алгебраической геометрии и нашла приложения в различных областях математики, которые до этого казались далекими от алгебраической геометрии. Мы уже отмечали доказательство Стенли условия необходимости в $g$-теореме для симплициальных многогранников. Другие замечательные приложения включают вычисление объемов и числа целых точек решеточных многогранников; взаимосвязи с многочленами Ньютона и особенностями, найденные в работах Хованского и Кушниренко; дискриминанты, результанты и гипергеометрические функции (по работам Гельфанда, Зелевинского и Капранова); рефлексивные многогранники и приложения к зеркальной симметрии для торических гиперповерхностей Калаби-Яо, найденные Батыревым и другими авторами. Стандартными ссылками по геометрии торических многообразий являются обзор Данилова [32] и книги Оды [63] и Фултона [40]. Более современный обзор Кокса [31] охватьвает новые приложения, включая упомянутые выше. В наши задачи не входит новый обзор по торической геометрии. В этом параграфе мы концентрируемся на некоторых топологических и комбинаторных аспектах теории торических многообразий. Мы также приводим доказательство Стенли условия необходимости в $g$-теореме.

Пусть $\mathbb{C}^{*}=\mathbb{C} \backslash\{0\}$ обозначает мультипликативную группу комплексных чисел. Произведение $n$ копий $\mathbb{C}^{*}$, обозначаемое $\left(\mathbb{C}^{*}\right)^{n}$, в теории алгебраических групп называется тором. В топологии тором $T^{n}$ называется произведение $n$ окружностей. Мы используем топологическую терминологию, а $\left(\mathbb{C}^{*}\right)^{n}$ мы будем называть алгебраическим тором. Тор $T^{n}$ стандартньм образом описьвается как подгруппа алгебраического тора $\left(\mathbb{C}^{*}\right)^{n}$ :

$$
T^{n}=\left\{\left(e^{2 \pi i \varphi_{1}}, \ldots, e^{2 \pi i \varphi_{n}}\right) \in \mathbb{C}^{n}\right\},
$$

где набор параметров $\left(\varphi_{1}, \ldots, \varphi_{n}\right)$ пробегает пространство $\mathbb{R}^{n}$.

ОПРЕДЕЛЕНИЕ 2.1.1. Торическое многообразие - это нормальное алгебраическое многообразие $M$, содержашее алгебраический тор $\left(\mathbb{C}^{*}\right)^{n}$ в качестве открытого 
по Зарискому подмножества таким образом, что естественное действие $\left(\mathbb{C}^{*}\right)^{n}$ на себе продолжается до действия на $M$.

Таким образом, $\left(\mathbb{C}^{*}\right)^{n}$ действует на $M$ с плотной орбитой.

Каждое торическое многообразие задается набором комбинаторных данных, а именно (рациональным многогранным) веером в некотором пространстве $\mathbb{R}^{n}$.

Пусть $\mathbb{R}^{n}$ - евклидово пространство и $\mathbb{Z}^{n} \subset \mathbb{R}^{n}$ - целочисленная решетка. Для любого конечного множества векторов $\boldsymbol{l}_{1}, \ldots, \boldsymbol{l}_{s} \in \mathbb{R}^{n}$ определим вылуклый многогранныци конус $\sigma$, порожденный $\boldsymbol{l}_{1}, \ldots, \boldsymbol{l}_{s}$, как

$$
\sigma=\left\{r_{1} \boldsymbol{l}_{1}+\cdots+r_{s} \boldsymbol{l}_{s} \in \mathbb{R}^{n}: r_{i} \geqslant 0\right\}
$$

Каждый выпукльй многогранньй конус является вьпуклым полиэдром в смысле определения 1.1.2. Таким образом, определены грани конуса. Конус $\sigma$ называется $p a-$ ииональным , если его образующие векторы $l_{1}, \ldots, l_{s}$ могут быть выбраны из решетки $\mathbb{Z}^{n}$, и строго выпуклым, если он не содержит никакой прямой, проходящей через начало координат. Все конусы, рассматриваемые ниже, являются строго выпуклыми и рациональными. Конус называется симплиииальным (соответственно неособым), если он порождается частью базиса пространства $\mathbb{R}^{n}$ (соответственно решетки $\mathbb{Z}^{n}$ ). Веером назьвается множество $\Sigma$ конусов в некотором пространстве $\mathbb{R}^{n}$ такое, что каждая грань конуса из $\Sigma$ также принадлежит $\Sigma$ и пересечение любых двух конусов из $\Sigma$ является гранью каждого из них. Веер $\Sigma$ назьвается симплиииальным. (соответственно неособым), если все конусы из $\Sigma$ симплициальны (соответственно неособы). Веер $\Sigma$ в $\mathbb{R}^{n}$ называется полным , если объединение всех конусов из $\Sigma$ есть все $\mathbb{R}^{n}$.

Каждый веер $\Sigma$ в $\mathbb{R}^{n}$ определяет торическое многообразие $M_{\Sigma}$ комплексной размерности $n$, структура орбит которого описьвается комбинаторикой веера $\Sigma$. А именно, $k$-мерные конусы веера $\Sigma$ соответствуют орбитам коразмерности $k$ действия алгебраического тора. В частности, $n$-мерные конусы соответствуют неподвижным точкам, а начало координат соответствует единственной плотной орбите. Торическое многообразие $M_{\Sigma}$ компактно тогда и только тогда, когда веер $\Sigma$ является полным. Если $\Sigma$ симплициален, то многообразие $M_{\Sigma}$ является орбифолдом (т.е. локально гомеоморфно фактор-пространству $\mathbb{R}^{2 n}$ по действию конечной группы). Наконец, если веер $\Sigma$ является неособым, то (как можно было бы догадаться из названия) многообразие $M_{\Sigma}$ является неособым (гладким).

Пусть $\Sigma$ - симплициальньй веер в $\mathbb{R}^{n}$ с $m$ одномерными конусами (или лучами). Выберем в качестве образующих этих $m$ лучей некоторые целочисленные примитивные (т.е. с взаимно простыми координатами) векторы $\boldsymbol{l}_{1}, \ldots, \boldsymbol{l}_{m}$. Веер $\Sigma$ определяет симплициальньй комплекс $K_{\Sigma}$ на множестве вершин $[m]$. По определению подмножество $\left\{i_{1}, \ldots, i_{k}\right\} \subset[m]$ является симплексом в $K_{\Sigma}$, если векторы $\boldsymbol{l}_{i_{1}}, \ldots, \boldsymbol{l}_{i_{k}}$ порождают некоторый конус из $\Sigma$. Легко видеть, что если веер $\Sigma$ полный, то $K_{\Sigma}$ является симплициальной $(n-1)$-сферой.

Обозначим $l_{i j}:=\left(\boldsymbol{l}_{j}\right)_{i}, 1 \leqslant i \leqslant n, 1 \leqslant j \leqslant m$. Таким образом, $\boldsymbol{l}_{j}=\left(l_{1 j}, \ldots, l_{n j}\right)^{t} \in$ $\mathbb{Z}^{n}$. Поставим в соответствие каждому вектору $l_{j}$ переменную $v_{j}$ степени 2 и определим линейные формы

$$
\theta_{i}:=l_{i 1} v_{1}+\cdots+l_{i m} v_{m} \in \mathbb{Z}\left[v_{1}, \ldots, v_{m}\right], \quad 1 \leqslant i \leqslant n
$$


Обозначим через $\mathscr{J}_{\Sigma}$ идеал в $\mathbb{Z}\left[v_{1}, \ldots, v_{m}\right]$, порожденньй этими линейными формами, т.е. $\mathscr{J}_{\Sigma}=\left(\theta_{1}, \ldots, \theta_{n}\right)$. Образы форм $\theta_{1}, \ldots, \theta_{n}$ и идеала $\mathscr{J}_{\Sigma}$ в кольце Стенли-Райснера $\mathbb{Z}\left(K_{\Sigma}\right)=\mathbb{Z}\left[v_{1}, \ldots, v_{m}\right] / \mathscr{I}_{K_{\Sigma}}$ (см. определение 1.3.1) мы будем обозначать теми же символами $\theta_{1}, \ldots, \theta_{n}$ и $\mathscr{J}_{\Sigma}$.

ТеОрема 2.1.2 (Данилов, Юркевич). Пусть $\Sigma-$ полный неособый веер в $\mathbb{R}^{n} u$ $M_{\Sigma}$ - соответствующее торическое многообразие. Тогда

(а) числа Бетти (ранги групп гомологий) многообразия $M_{\Sigma}$ равньи нулю в нечетных размерностях, а в четных размерностях задаются формулой

$$
b^{2 i}\left(M_{\Sigma}\right)=h_{i}\left(K_{\Sigma}\right), \quad i=0,1, \ldots, n,
$$

где $\boldsymbol{h}\left(K_{\Sigma}\right)=\left(h_{0}, \ldots, h_{n}\right)-h$-вектор комплекса $K_{\Sigma}$;

(б) замыкания орбит, соответствующих 1-мерныц конусам веера $\Sigma$, являются подмногообразиями коразмерности 2 (дивизорами) $D_{i}$ многообразия $M_{\Sigma}$. Пусть $v_{i}, 1 \leqslant i \leqslant m,-$ соответствующие 2 -мерные классы когомологий. Тогда кольио когомологий $M_{\Sigma}$ есть

$$
H^{*}\left(M_{\Sigma} ; \mathbb{Z}\right) \cong \mathbb{Z}\left[v_{1}, \ldots, v_{m}\right] /\left(\mathscr{I}_{K_{\Sigma}}+\mathscr{J}_{\Sigma}\right)=\mathbb{Z}\left(K_{\Sigma}\right) / \mathscr{J}_{\Sigma} .
$$

Кроме того, $\theta_{1}, \ldots, \theta_{n}$ является регулярной последовательностью в $\mathbb{Z}\left(K_{\Sigma}\right)$.

Эта теорема была доказана Юркевичем для проективных гладких торических многообразий и Даниловым [32; теорема 10.8] в общем случае. Заметим, что первая часть теоремы 2.1.2 вытекает из второй части и леммы 1.3.3.

Теорема 2.1.2 показьвает, что кольцо когомологий многообразия $M_{\Sigma}$ отождествляется с кольиом Чжоу [40; $§ 5.1]$ и порождено двумерными классами. Заметим, что идеал $\mathscr{I}_{K_{\Sigma}}$ зависит лишь от симплициального комплекса $K_{\Sigma}$ (т.е. от решетки пересечений веера), в то время как идеал $\mathscr{J}_{\Sigma}$ зависит от самого веера.

ЗАмЕчАниЕ. Как было показано Даниловьм, теорема 2.1.2 имеет место также для симплициальных вееров и определяемых ими торических многообразий, если заменить кольцо коэффициентов $\mathbb{Z}$ на любое поле нулевой характеристики (например, $\mathbb{Q}$ ).

КонСтрукция 2.1.3 (нормальньй веер и торические многообразия, возникаюшие из многогранников). Пусть дан $n$-многогранник (1) с вершинами в точках целочисленной решетки $\mathbb{Z}^{n} \subset \mathbb{R}^{n}$ (такие многогранники назьваются целочисленными или решеточнымм ). Тогда векторы $l_{i}$ в $(1), 1 \leqslant i \leqslant m$, могут быть выбраны целочисленными и примитивными, а числа $a_{i}$ могут быть выбраны целыми. Заметим, что кажщьй $\boldsymbol{l}_{i}$ представляет собой нормальньй вектор гиперграни $F_{i} \subset P^{n}$, направленньй внутрь многогранника. Определим полньй веер $\Sigma(P)$, конусы которого порождены такими наборами нормальных векторов $\boldsymbol{l}_{i_{1}}, \ldots, \boldsymbol{l}_{i_{k}}$, что соответствуюшие гиперграни $F_{i_{1}}, \ldots, F_{i_{k}}$ имеют непустое пересечение в $P ; \Sigma(P)$ назьвается нормальным веером многогранника $P$. По-другому, если $0 \in P$, то нормальный веер состоит из конусов над гранями полярного многогранника $P^{*}$. Определим торическое многообразие $M_{P}:=M_{\Sigma(P)}$. Многообразие $M_{P}$ является гладким тогда и только тогда, когда $P$ простой многогранник и нормальные векторы $\boldsymbol{l}_{i_{1}}, \ldots, \boldsymbol{l}_{i_{n}}$ любого набора из $n$ гиперграней $F_{i_{1}}, \ldots, F_{i_{n}}$, сходяшихся в одной вершине, образуют базис решетки $\mathbb{Z}^{n}$. 
ЗАМЕЧАНИЕ. Любой комбинаторньй простой многогранник яВляется рациональным, т.е. допускает геометрическую реализацию с рациональными (или, эквивалентно, целыми) координатами вершин. Действительно, небольшое возмушение определяющих неравенств в (1), делающее их рациональными, не меняет комбинаторного типа (так как полупространства, определяемые неравенствами, находятся в общем положении). В результате мы получаем простой многогранник $P^{\prime}$ того же комбинаторного типа, но с рациональными координатами вершин. Для того чтобы получить реализацию с целыми координатам вершин, надо рассмотреть увеличенный многогранник $k P^{\prime}$ с соответствуюшим коэффициентом $k \in \mathbb{Z}$. Достаточно неожиданньпм фактом является то, что существуют нерациональные выпуклые многогранники (не простые и не симплициальные), см. [89; пример 6.21]. Возвращаясь к простьм многогранникам, заметим, что различные реализации данного комбинаторного простого многогранника как решеточного многогранника могут давать различные (даже топологически) торические многообразия $M_{P}$. В то же время существуют комбинаторные простые многогранники, которые не допускают ни одной геометрической реализации с гладким $M_{P}$. Мы приведем один из таких примеров в следуюшем параграфе (см. пример 2.2.14).

Далее в этом параграфе все многогранники предполагаются простыми. Конструкция 2.1.3 позволяет построить симплициальньй веер $\Sigma(P)$ и торическое многообразие $M_{P}$ по любому решеточному простому многограннику $P$. При этом, однако, многогранник $P$ содержит больше информации, чем веер $\Sigma(P)$. Действительно, кроме нормальных векторов $\boldsymbol{l}_{i}$, мы также имеем числа $a_{i} \in \mathbb{Z}, 1 \leqslant i \leqslant m$ (см. (1)). Линейная комбинация $D=a_{1} D_{1}+\cdots+a_{m} D_{m}$ (см. теорему 2.1.2) является обильньлм дивизором. Этот дивизор определяет проективное вложение $M_{P} \subset \mathbb{C} P^{r}$ для некоторого $r$ (которое можно взять равным числу вершин многогранника $P$ ). Таким образом, все торические многообразия, возникающие из многогранников, являются проективными. Обратно, если имеется гладкое проективное торическое многообразие $M \subset \mathbb{C} P^{r}$, то мы имеем очень обильный дивизор (линейное расслоение) $D$ гиперплоского сечения, нулевые когомологии которого порождены сечениями, соответствующими цельм точкам внутри некоторого простого многогранника $P$. Для этого $P$ мы имеем $M=M_{P}$. Пусть $\omega:=a_{1} v_{1}+\cdots+a_{m} v_{m} \in H^{2}\left(M_{P} ; \mathbb{Q}\right)$ - класс когомологий $D$.

Теорема 2.1.4 (сильная теорема Лефшеца для торических многообразий). Пусть $P^{n}$ - решеточный простой многогранник (1), $M_{P}$ - проективное торическое многообразие, определяемое $P, u \omega=a_{1} v_{1}+\cdots+a_{m} v_{m} \in H^{2}\left(M_{P} ; \mathbb{Q}\right)$. Тогда отображения

$$
H^{n-i}\left(M_{P} ; \mathbb{Q}\right) \stackrel{\cdot \omega^{i}}{\longrightarrow} H^{n+i}\left(M_{P} ; \mathbb{Q}\right), \quad 1 \leqslant i \leqslant n
$$

являются изоморфизмами.

Из проективности вытекает, что если $M_{P}$ гладко, то оно является кэлеровым многообразием и $\omega$ - класс кэлеровой 2-формы.

ЗАмЕчАнИЕ. Если заменить обычные когомологии на когомологии пересечений Горески-Макферсона, то теорема 2.1.4 имеет место для любого проективного торического многообразия, а не только для многообразий, происходящих из простых решеточных многогранников (см. обсуждение в [40; 55.2$])$. 
ПримеР 2.1.5. Комплексноепроективное пространство $\mathbb{C} P^{n}=\left\{\left(z_{0}: z_{1}: \cdots: z_{n}\right)\right.$, $\left.z_{i} \in \mathbb{C}\right\}$ является торическим многообразием. $\left(\mathbb{C}^{*}\right)^{n}$ действует на $\mathbb{C} P^{n}$ как $\left(t_{1}, \ldots, t_{n}\right)$. $\left(z_{0}: z_{1}: \cdots: z_{n}\right)=\left(z_{0}: t_{1} z_{1}: \cdots: t_{n} z_{n}\right)$. Очевидно, $\left(\mathbb{C}^{*}\right)^{n} \subset \mathbb{C}^{n} \subset \mathbb{C P}^{n}$ является открытым всюду плотньп подмножеством. Веер, определяющий $\mathbb{C} P^{n}$, состоит из конусов, порожденных всеми собственными подмножествами множества из $(n+1)$ векторов $e_{1}, \ldots, e_{n},-e_{1}-\cdots-e_{n}$ в $\mathbb{R}^{n}$. Теорема 2.1.2 отождествляет кольцо когомологий $H^{*}\left(\mathbb{C} P^{n} ; \mathbb{Z}\right)=\mathbb{Z}[u] /\left(u^{n+1}\right), \operatorname{dim} u=2$, с фактор-кольцом $\mathbb{Z}\left[v_{1}, \ldots, v_{n+1}\right] /\left(v_{1} \cdots v_{n+1}\right.$, $\left.v_{1}-v_{n+1}, \ldots, v_{n}-v_{n+1}\right)$. Торическое многообразие $\mathbb{C} P^{n}$ возникает из многогранника: $\mathbb{C} P^{n}=M_{P}$, где $P$ - стандартньй $n$-симплекс $(2)$. Класс $\omega \in H^{2}\left(\mathbb{C} P^{n} ; \mathbb{Q}\right)$ из теоремы 2.1.4 в данном случае есть $\omega=v_{n+1}$.

Теперь мы можем привести доказательство Стенли условия необходимости в $g$-теореме для симплициальных многогранников.

ДОКАЗАТЕЛЬСТВО УСЛОВИЯ НЕОБХОДИМОСТИ В ТЕОРЕмЕ 1.1.17. Реализуем простой многогранник как решеточньй многогранник $P^{n} \subset \mathbb{R}^{n}$. Пусть $M_{P}-$ соответствующее торическое многообразие. Часть (а) уже доказана (теорема 1.1.11). Из теоремы 2.1.4 вытекает, что умножение на $\omega \in H^{2}\left(M_{P} ; \mathbb{Q}\right)$ является мономорфизмом $H^{2 i-2}\left(M_{P} ; \mathbb{Q}\right) \rightarrow H^{2 i}\left(M_{P} ; \mathbb{Q}\right)$ при $i \leqslant\left[\frac{n}{2}\right]$. Это вместе с частью (б) теоремы 2.1 .2 дает $h_{i-1} \leqslant h_{i}, 0 \leqslant i \leqslant\left[\frac{n}{2}\right]$, и тем самым часть (б) доказана. Для доказательства части (в) введем градуированную коммутативную $\mathbb{Q}$-алгебру $A:=H^{*}\left(M_{P} ; \mathbb{Q}\right) /(\omega)$. Тогда $A^{0}=\mathbb{Q}, A^{2 i}=H^{2 i}\left(M_{P} ; \mathbb{Q}\right) / \omega \cdot H^{2 i-2}\left(M_{P} ; \mathbb{Q}\right)$ при $1 \leqslant i \leqslant\left[\frac{n}{2}\right]$ и $A$ порождена элементами степени два (так как это вьполнено для $H^{*}\left(M_{P} ; \mathbb{Q}\right)$ ). Из теоремы 1.1.18 вытекает, что числа $\operatorname{dim} A^{2 i}=h_{i}-h_{i-1}, 0 \leqslant i \leqslant\left[\frac{n}{2}\right]$, являются компонентами $M$-вектора, что доказывает (в) и всю теорему.

ЗАмечАниЕ. Соотношения Дена-Соммервилля теперь также вытекают из двойственности Пуанкаре для $M_{P}$ (которая также имеет место для особых $M_{P}$ при условии, что $P$ - простой многогранник).

Теперь мы рассмотрим действие тора $T^{n} \subset\left(\mathbb{C}^{*}\right)^{n}$ на неособом компактном торическом многообразии $M$. Это действие локально эквивалентно стандартному действию $T^{n}$ на $\mathbb{C}^{n}$ (точное определение приведено в следующем параграфе). Пространство орбит $M / T^{n}$ гомеоморфно $n$-мерному шару, снабженному топологической структурой многообразия с углами при помоши множеств неподвижных точек соответствующих торических подгрупп, см. [40; $\$ 4.1]$. Многообразие с углами представляет собой пространство, окрестность каждой точки которого моделируется некоторым открытьм подмножеством положительного конуса $\mathbb{R}_{+}^{n}$, см. (4). Из этого нестрогого описания несложно восстановить точное определение, см. [49], которое мы не приводим ввиду его некоторой громоздкости.

Конструкция 2.1.6. Пусть $P^{n}$ - простой многогранник. Для каждой вершины $v \in P^{n}$ обозначим через $U_{v}$ открытое подмножество в $P^{n}$, получаемое удалением всех граней, не содержащих $v$. Очевидно, $U_{v}$ диффеоморфно $\mathbb{R}_{+}^{n}$ (и даже аффинно изоморфно некоторому открытому подмножеству в $\mathbb{R}_{+}^{n}$, содержащему 0 ). Отсюда вытекает, что $P^{n}$ является многообразием с углами, с атласом $\left\{U_{v}\right\}$.

Если $M=M_{P}$ возникает из некоторого (простого) многогранника $P^{n}$, то пространство орбит $M / T^{n}$ диффеоморфно $P^{n}$ как многообразие с углами. Более того, имеется явное отображение $M_{P} \rightarrow \mathbb{R}^{n}$ (отображсение моментов), образом ко- 
торого является $P^{n}$, а слоями - $T^{n}$-орбиты $[40 ; \S 4.2]$ (мы рассмотрим взаимосвязи с отображениями моментов и некоторьми аспектами симплектической геометрии более детально в $\S 5.2)$. При этом отображении внутренность грани коразмерности $k$ отождествляется с множеством орбит, имеющих одну и ту же стационарную подгруппу размерности $k$. В частности, действие свободно над внутренностью многогранника. Рассматриваемое как гладкое многообразие, $M_{P}$ отождествляется с фактор-пространством $T^{n} \times P^{n} / \sim$ по некоторому отношению эквивалентности $\sim$. Такое описание действия тора на неособом торическом многообразии привело к созданию содержательного топологического аналога теории алгебраических торических многообразий - теории квазиторических многообразий.

$\S$ 2.2. Квазиторические многообразия. Квазиторическое многообразие ${ }^{3}$ представляет собой гладкое многообразие с действием тора, свойства которого аналогичны свойствам действия (компактного) тора на неособом проективном торическом многообразии. Это понятие появилось в [33]. В последующих определениях мы следуем этой работе, принимая во внимание ряд существенных доработок из [26].

Как и в предыдущем параграфе, мы рассматриваем тор $T^{n}$ как стандартную подгруппу $(23)$ в $\left(\mathbb{C}^{*}\right)^{n}$, тем самым фиксируя ориентацию и координатные подгрупшы $T_{i} \cong S^{1}, i=1, \ldots, n$, в $T^{n}$. Мы будем назьвать представление тора $T^{n}$ диагональными матрицами в $U(n)$ стандартным действием на $\mathbb{C}^{n}$. Пространство орбит этого действия представляет собой положительньй конус $\mathbb{R}_{+}^{n}$. Каноническая проекция

$$
T^{n} \times \mathbb{R}_{+}^{n} \rightarrow \mathbb{C}^{n}:\left(t_{1}, \ldots, t_{n}\right) \times\left(x_{1}, \ldots, x_{n}\right) \rightarrow\left(t_{1} x_{1}, \ldots, t_{n} x_{n}\right)
$$

позволяет отождествить $\mathbb{C}^{n}$ с фактор-пространством $T^{n} \times \mathbb{R}_{+}^{n} / \sim$ по отношению эквивалентности $\sim$, которое далее будет играть важную роль.

Пусть $M^{2 n}-2 n$-мерное гладкое многообразие с действием тора $T^{n}$. Скажем, что действие $T^{n}$ является локально стандартным, если любая точка $x \in M^{2 n}$ лежит в некоторой $T^{n}$-инвариантной окрестности $U(x)$, для которой существует $\psi$-эквивариантный гомеоморфизм $f: U(x) \rightarrow W$ на некоторое $\left(T^{n}\right.$-инвариантное) открытое подмножество $W \subset \mathbb{C}^{n}$. Это означает, что сушествует автоморфизм $\psi: T^{n} \rightarrow T^{n}$ такой, что $f(t \cdot y)=\psi(t) f(y)$ для любых $t \in T^{n}, y \in U(x)$. Пространство орбит для локально стандартного действия тора на $M^{2 n}$ является $n$-мерньм многообразием с углами. Квазиторические многообразия соответствуют важному частному случаю, ког да пространство орбит диффеоморфно, как многообразие с углами, некоторому простому многограннику $P^{n}$.

ОПРЕДЕЛЕНИЕ 2.2.1. Пусть $P^{n}$ - простой многогранник. Многообразие $M^{2 n}$ с локально стандартньм действием тора $T^{n}$ назьвается квазиторическим многообразием над $P^{n}$, если существует проекция $\pi: M^{2 n} \rightarrow P^{n}$, слоями которой являются орбиты действия.

При проекции $\pi$ точки, имеющие одну и ту же стационарную подгруппу коразмерности $k$, отображаются во внутренность некоторой $k$-грани многогранника $P^{n}$. В частности, действие тора свободно над внутренностью многогранника, в то время как вершины $P^{n}$ соответствуют неподвижным точкам многообразия $M^{2 n}$.

\footnotetext{
${ }^{3}$ В англоязычной литературе также используется термин toric manifold.
} 
ЗАмЕчАниЕ. Два простых многогранника комбинаторно эквивалентны тогда и только тогда, когда они диффеоморфны как многообразия с углами.

Предположим, $P^{n}$ имеет $m$ гиперграней $F_{1}, \ldots, F_{m}$. Для каждой гиперграни $F_{i}$ прообраз $\pi^{-1}\left(F_{i}\right)$ является подмногообразием $M_{i}^{2(n-1)} \subset M^{2 n}$ с одномерной стационарной подгруппой $T\left(F_{i}\right)$ в $T^{n}$, которая описывается следующим образом:

$$
T\left(F_{i}\right)=\left\{\left(e^{2 \pi i \lambda_{1 i} \varphi}, \ldots, e^{2 \pi i \lambda_{n i} \varphi}\right) \in T^{n}\right\}
$$

где $\varphi \in \mathbb{R}$ и $\boldsymbol{\lambda}_{i}=\left(\lambda_{1 i}, \ldots, \lambda_{n i}\right)^{t} \in \mathbb{Z}^{n}$ - некоторьй примитивный вектор. Этот вектор $\boldsymbol{\lambda}_{i}$ определяется подгруппой $T\left(F_{i}\right)$ лишш с точностью до знака. Выбор знака задает ориентацию для $T\left(F_{i}\right)$. В настоящий момент мы не будем заботиться об этом знаке и выберем его произвольно. Более детальное исследование вопросов, связанных с выбором этих знаков, является предметом следующего параграфа. Мы будем назьвать $\lambda_{i}$ вектором гиперграни $F_{i}$. Действие тора $T^{n} / T\left(F_{i}\right)$ на $M_{i}$ описьвает $M_{i}$ как квазиторическое многообразие над $F_{i}$. Соответствие

$$
\ell: F_{i} \mapsto T\left(F_{i}\right)
$$

назьвается характеристическим отображением многообразия $M^{2 n}$. Предположим, гранњ $G^{n-k}$ коразмерности $k$ записана как пересечение $k$ гиперграней: $G^{n-k}=$ $F_{i_{1}} \cap \cdots \cap F_{i_{k}}$. Тогда подмногообразия $M_{i_{1}}, \ldots, M_{i_{k}}$ пересекаются трансверсально по некоторому подмногообразию $M(G)^{2(n-k)}$. Отображение $T\left(F_{i_{1}}\right) \times \cdots \times T\left(F_{i_{k}}\right) \rightarrow T^{n}$ инъективно, так как $T\left(F_{i_{1}}\right) \times \cdots \times T\left(F_{i_{k}}\right)$ отождествляется с $k$-мерной стационарной подгруппой многообразия $M(G)^{2(n-k)}$. Отсюда вытекает, что векторы $\boldsymbol{\lambda}_{i_{1}}, \ldots, \boldsymbol{\lambda}_{i_{k}}$ образуют часть базиса решетки $\mathbb{Z}^{n}$.

Рассмотрим целочисленную $(n \times m)$-матрицу $\Lambda, i$-й столбец которой образован координатами вектора $\boldsymbol{\lambda}_{i}, i=1, \ldots, m$. Каждая вершина $v \in P^{n}$ есть пересечение $n$ гиперграней: $v=F_{i_{1}} \cap \cdots \cap F_{i_{n}}$. Пусть $\Lambda_{(v)}:=\Lambda_{\left(i_{1}, \ldots, i_{n}\right)}$ - максимальный минор матрицы $\Lambda$, образованньй столбцами $i_{1}, \ldots, i_{n}$. Тогда

$$
\operatorname{det} \Lambda_{(v)}= \pm 1
$$

Соответствие

$$
G^{n-k} \mapsto \text { стационарная подгруппа многообразия } M(G)^{2(n-k)}
$$

продолжает характеристическое отображение (25) до отображения решетки граней многогранника $P^{n}$ в решетку торических подгрупп тора $T^{n}$.

Как и в случае стандартного действия тора $T^{n}$ на $\mathbb{C}^{n}$, имеется проекция $T^{n} \times P^{n}$ $\rightarrow M^{2 n}$, слой которой над $x \in M^{2 n}$ имеет вид (стационарная подгруппа точки $\left.x\right) \times$ (орбита точки $x$ ). Это соображение может быть использовано для восстановления квазиторического многообразия по любой заданной паре $\left(P^{n}, \ell\right)$, где $P^{n}-($ комбинаторньй) простой многогранник, а $\ell$ - отображение из множества гиперграней многогранника $P^{n}$ в одномерные подгруппы тора $T^{n}$ такое, что $\ell\left(F_{i_{1}}\right) \times \cdots \times \ell\left(F_{i_{k}}\right) \rightarrow T^{n}$ инъективно при условии $F_{i_{1}} \cap \cdots \cap F_{i_{k}} \neq \varnothing$. Такая пара $\left(P^{n}, \ell\right)$ называется характеристической парой. Отображение $\ell$ непосредственно продолжается до отображения решетки граней многогранника $P^{n}$ в решетку торических подгрупп тора $T^{n}$. 
Конструкция 2.2.2 (построение квазиторического многообразия по характеристической паре). Заметим, что каждая точка $q$ многогранника $P^{n}$ лежит во внутренности единственной грани $G(q)$. Рассмотрим фактор-пространство $\left(T^{n} \times P^{n}\right) / \sim$, где $\left(t_{1}, q\right) \sim\left(t_{2}, q\right)$ тогда и только тогда, когда $t_{1} t_{2}^{-1}$ принадлежит подгруппе $\ell(G(q))$. Свободное действие тора $T^{n}$ на $T^{n} \times P^{n}$ определяет действие на $\left(T^{n} \times P^{n}\right) / \sim$ с пространством орбит $P^{n}$. Последнее действие свободно над внутренностью многогранника и имеет по одной неподвижной точке для каждой вершины. Аналогично тому, как многогранник $P^{n}$ покрывается открытыми множествами $U_{v}$, диффеоморфными $\mathbb{R}_{+}^{n}$ (см. конструкцию 2.1.6), пространство $\left(T^{n} \times P^{n}\right) / \sim$ покрывается открытыми множествами $\left(T^{n} \times U_{v}\right) / \sim$, гомеоморфными $\left(T^{n} \times \mathbb{R}_{+}^{n}\right) / \sim$ или $\mathbb{C}^{n}$. Отсюда вытекает, что действие тора на $\left(T^{n} \times P^{n}\right) / \sim$ локально стандартно и, следовательно, $M^{2 n}(\ell):=\left(T^{n} \times P^{n}\right) / \sim$ является квазиторическим многообразием.

Пусть $\psi: T^{n} \rightarrow T^{n}$ - некоторьй автоморфизм. Скажем, что два квазиторических многообразия $M_{1}^{2 n}, M_{2}^{2 n}$ над одним многогранником $P^{n}$ являются $\psi$-әквивариантно диффеоморфными, если сушествует диффеоморфизм $f: M_{1}^{2 n} \rightarrow M_{2}^{2 n}$ такой, что $f(t \cdot x)=\psi(t) f(x)$ для всех $t \in T^{n}, x \in M_{1}^{2 n}$. Автоморфизм $\psi$ индуцирует автоморфизм $\psi_{*}$ решеток торических подгрупп тора $T^{n}$. Каждый такой автоморфизм определяет $\psi$-преобразование характеристических пар, при котором два характеристических отображения отличаются на $\psi_{*}$. Следующее предложение доказано в [26; предложение 2.6] и обобщает предложение 1.8 из [33].

ПРЕДЛОЖЕНИЕ 2.2.3. ДЛя любого автоморфизма $\psi$ конструкиия 2.2 .2 определяет биекиию между классами $\psi$-эквивариантно диффеоморфных квазиторических многообразий и $\psi$-преобразованиями пар $\left(P^{n}, \ell\right)$.

Если $\psi$ - тождественный автоморфизм, то мы получаем, что два квазиторических многообразия эквивариантно диффеоморфны тогда и только тогда, когда их характеристические пары совпадают.

Ниже мы, следуя [33], строим клеточное разбиение многообразия $M^{2 n}$, имеющее клетки лишь в четных размерностях, и выгисляем его числа Бетти.

КонстрУкция 2.2.4. Вспомним “идеи из теории Морса", использованные при доказательстве соотношений Дена-Соммервилля (теоремы 1.1.11). Там мы превратили 1 -остов многогранника $P^{n}$ в ориентированный граф̆ и определили индекс $\operatorname{ind}(v)$ вершины $v \in P^{n}$ как число ребер, направленных к $v$. Эти ребра порождают гранњ $G_{v}$ размерности $\operatorname{ind}(v)$. Обозначим через $\widehat{G}_{v}$ подмножество грани $G_{v}$, получаемое удалением из нее всех граней, не содержаших $v$. Очевидно, $\widehat{G}_{v}$ диффеоморфно $\mathbb{R}_{+}^{\text {ind }(v)}$ и содержится в открытом множестве $U_{v} \subset P^{n}$ из конструкции 2.1.6. Тогда $e_{v}:=\pi^{-1} \widehat{G}_{v}$ отождествляется с $\mathbb{C}^{\text {ind }(v)}$ и объединение подмножеств $e_{v}$ по всем вершинам многогранника определяет клеточное разбиение многообразия $M^{2 n}$. Заметим, что все клетки имеют четную размерность и замыкание клетки $e_{v}$ есть в точности подмногообразие $M\left(G_{v}\right)^{2} \operatorname{ind}(v) \subset M^{2 n}$. Эта конструкция была ранее применена Хованским [50] для построения клеточных разбиений торических многообразий. 
ПРЕДЛОЖЕНИЕ 2.2.5. Числа Бетти многообразия $M^{2 n}$ равны нулю в нечетных размерностях, а в четных размерностях находятся как

$$
b_{2 i}\left(M^{2 n}\right)=h_{i}\left(P^{n}\right), \quad i=0,1, \ldots, n,
$$

где $\boldsymbol{h}\left(P^{n}\right)=\left(h_{0}, \ldots, h_{n}\right)-h$-вектор многогранника $P^{n}$.

ДокАЗАТЕльство. Число Бетти $b_{2 i}\left(M^{2 n}\right)$ равно числу $2 i$-мерных клеток в построенном выше клеточном комплексе. Последнее число равно числу вершин индекса $i$, что, в свою очередь, есть $h_{i}\left(P^{n}\right)$, как показано при доказательстве теоремы 1.1.11.

Для любого квазиторического многообразия $M^{2 n}$ с характеристическим отображением (25) и векторами гиперграней $\boldsymbol{\lambda}_{i}=\left(\lambda_{1 i}, \ldots, \lambda_{n i}\right)^{t} \in \mathbb{Z}^{n}, i=1, \ldots, m$, определим линейные формы

$$
\theta_{i}:=\lambda_{i 1} v_{1}+\cdots+\lambda_{i m} v_{m} \in \mathbb{Z}\left[v_{1}, \ldots, v_{m}\right], \quad 1 \leqslant i \leqslant n .
$$

Образы этих линейных форм в кольце граней $\mathbb{Z}\left(P^{n}\right)$ будут обозначаться теми же буквами.

Лемма 2.2.6 (Дэвис, Янушкевич). Для любого квазиторического многообразия $M^{2 n}$ над $P^{n}$ последовательность $\theta_{1}, \ldots, \theta_{n}$ является регулярной последовательностью (степени два) в кольие $\mathbb{Z}\left(P^{n}\right)$.

Пусть $\mathscr{J}_{\ell}$ обозначает идеал в кольце $\mathbb{Z}\left(P^{n}\right)$, порожденный элементами $\theta_{1}, \ldots, \theta_{n}$.

Теорема 2.2.7 (Дэвис, Янушкевич). Пусть $v_{i}, 1 \leqslant i \leqslant m,-$ двумерные классы когомологий, двойственные $\kappa$ подмногообразиям $M_{i}^{2(n-1)} \subset M^{2 n}$. Тогда кольио когомологий многообразия $M^{2 n}$ есть

$$
H^{*}\left(M^{2 n} ; \mathbb{Z}\right) \cong \mathbb{Z}\left[v_{1}, \ldots, v_{m}\right] /\left(\mathscr{I}_{P}+\mathscr{J}_{\ell}\right)=\mathbb{Z}\left(P^{n}\right) / \mathscr{J}_{\ell}
$$

Мы приводим доказательство двух предыдуших утверждений в $§ 3.4$.

ЗАмЕчАнИЕ. Изменение знака вектора $\boldsymbol{\lambda}_{i}$ соответствует переходу от $v_{i} \mathrm{~K}-v_{i}$ в описании кольца когомологий из теоремы 2.2.7. Этот факт будет иметь принципиальное значение в следующем параграфе.

ПримеР 2.2.8. Неособое проективное торическое многообразие $M_{P}$, возникающее из решеточного простого многогранника $P^{n}$, является квазиторическим многообразием над $P^{n}$. Соответствуюшее характеристическое отображение $\ell: F_{i} \mapsto T\left(F_{i}\right)$ определяется формулой $(24)$, где $\boldsymbol{\lambda}_{i}=\left(\lambda_{1 i}, \ldots, \lambda_{n i}\right)^{t}$ суть нормальные векторы $\boldsymbol{l}_{i}$ гиперграней многогранника $P^{n}, i=1, \ldots, m$ (см. (1)). Соответствующая характеристическая $(n \times m)$-матрица $\Lambda$ совпадает с матрицей $L$ из конструкции 1.1.4. В частности, если $P^{n}$ есть стандартный симплекс $\Delta^{n}(2)$, то $M_{P}$ есть $\mathbb{C} P^{n}$ (пример 2.1.5), а $\Lambda=(E \mid-\mathbf{1})$, где $E$ - единичная $(n \times n)$-матрица и $\mathbf{1}-$ столбец из единиц. См. также пример 2.4.14 ниже.

В общем случае неособое торическое многообразие не обязано быть квазиторическим: хотя пространство орбит (по действию $T^{n}$ ) является многообразием с углами (см. §2.1), оно может не быть диффеоморфным (или комбинаторно эквивалентным) 
какому-либо простому многограннику. Авторы благодарны Н. Стрикланду за то, что он обратил наше внимание на этот факт. Тем не менее, нам не известны соответствующие примеры. В [40; с. 71] приведен пример полного неособого веера $\Sigma$ в $\mathbb{R}^{3}$, который не может быть получен путем взятия конусов с вершиной 0 над гранями геометрического симплищиального многогранника. При этом, однако, соответствующий симплициальньй комплекс $K_{\Sigma}$ (являясь симплициальной 2-сферой) комбинаторно эквивалентен некоторой многогранной 2-сфере. Это означает, что неособое торическое многообразие $M_{\Sigma}$, не будучи проективньм, тем не менее является квазиторическим многообразием.

ПРОБЛЕМА 2.2.9. Построить пример неособого торического многообразия, которое не является квазиторическим многообразием.

Решение этой проблемы представляется авторам несложным и сводится к построению полного неособого веера $\Sigma$, ассоциированньй симплициальный комплекс $K_{\Sigma}$ которого является сферой Барнетта или любой другой $P L$-сферой, не являющейся многогранной сферой (см. $§ 1.2)$. Как было указано Н. Стрикландом, определение квазиторического многообразия может быть видоизменено таким образом, чтобы все неособые торические многообразия автоматически становились квазиторическими многообразиями. Для этого в определении 2.2.1 необходимо заменить простой многогранник на многогранный комплекс, двойственный к некоторой симплициальной сфере. Соответствующие конструкции в настоящее время находятся в стадии разработки.

С другой стороны, легко построить пример квазиторического многообразия, которое не является торическим. Простейшим из таких примеров является многообразие $\mathbb{C} P^{2}$ \# $\mathbb{C} P^{2}$ (связная сумма двух экземпляров $\mathbb{C} P^{2}$ ), которое является квазиторическим многообразием над квадратом $I^{2}$ (это следует из конструкции эквивариантной связной суммы, см. [33; 1.11], а также $\S 2.3$ и следствие 2.5.5 ниже), но не допускает даже почти комплексной структуры (комплексной структуры в касательном расслоении). В связи с этим возникает следующая проблема.

ПРоБЛЕМА 2.2.10. Пусть $P^{n}$ - простой многогранник с $m$ гипергранями, $\ell-$ характеристическое отображсние (25) и $M^{2 n}(\ell)$ - построенное по ней квазиторическое многообразие (конструкиия 2.2.2). Найти необходимые и достаточнье условия на $P^{n}$ и $\ell$, обеспечивающие существование $T^{n}$-инвариантной комплексной (соответственно почти комплексной) структуры на $M^{2 n}(\ell)$.

В почти комплексном случае последняя проблема была сформулирована в [33; проблема 7.6]. Пример 2.2.8 (характеристические отображения решеточных многогранников) дает достаточное условие для проблемы 2.2.10 (так как неособое торическое многообразие является комплексным). Тем не менее, это условие, очевидно, не является необходимым даже для существования комплексной структуры. Действительно, имеются неособые (непроективные) торические многообразия, которые не происходят из решеточных простых многогранников (см. уже упоминавшийся пример из [40; с. 71]). В то же время, нам не известны примеры не торических комплексных квазиторических многообразий.

ПроБЛЕма 2.2.11. Построить пример не торического квазиторического многообразия, допускающего $T^{n}$-инвариантную комплексную структуру. 
Хотя общее квазиторическое многообразие может не быть комплексным или почти комплексным, оно всегда допускает $T^{n}$-инвариантную комплексную структуру в $\mathrm{cma}$ бильном касательном расслоении. Соответствуюшие конструкции изложены в следуюшем параграфе.

Другой класс проблем связан с классификацией квазиторических многообразий над заданным комбинаторным простым многогранником. Общая задача классификации рассмотрена в $\S 2.5$. Как показывает пример 2.2.14 ниже, существуют комбинаторные простые многогранники, которые не допускают характеристического отображения (и тем самым не могут быть пространствами орбит для квазиторических многообразий).

ПроБЛема 2.2.12. Дать комбинаторное описание многогранников $P^{n}$, допускающих хотя бы одно характеристическое отображение (25).

Обобщение этой проблемы приведено в $§ 4.4$ (проблема 4.4.1).

Характеристическое отображение определяется целочисленной $(n \times m)$-матрицей $\Lambda$, удовлетворяюшей условию (26) для любой вершины $v \in P^{n}$. Условие $\left(\operatorname{det} \Lambda_{(v)}\right)^{2}=1$ выделяет гиперповерхность в пространстве $\mathscr{M}(n, m ; \mathbb{Z})$ целочисленных $(n \times m)$-матриц. Проблема 2.2.12 допускает следуюшую очевидную переформулировку.

ПРЕДЛОЖЕНИЕ 2.2.13. Множество всех характеристических матрии представляет собой пересечение гиперповерхностей

$$
\bigcap_{v \in P^{n}}\left\{\left(\operatorname{det} \Lambda_{(v)}\right)^{2}=1\right\}
$$

в пространстве $\mathscr{M}(n, m ; \mathbb{Z})$, где v пробегает множсество вериин многогранникa $P^{n}$.

ПримеР 2.2.14 (многогранник, не допускаюший характеристического отображения, [33; пример 1.22]). Пусть $P^{n}-2$-смежностньй простой многогранник с $m \geqslant 2^{n}$ гипергранями (например, полярный многогранник для циклического многогранника $C^{n}(m)$ (пример 1.1.9) с $n \geqslant 4$ и $m \geqslant 2^{n}$ ). Тогда $P^{n}$ не допускает ни одного характеристического отображения и, следовательно, не может быть пространством орбит никакого квазиторического многообразия. Действительно, согласно предложению 2.2.13 достаточно доказать, что пересечение (28) пусто. Так как $m \geqslant 2^{n}$, у любой матрицы $\Lambda \in \mathscr{M}(n, m ; \mathbb{Z})$ (без нулевых столбцов) найдутся два столбца, скажем $i$-й и $j$-й, совпадающие по модулю 2 . Так как $P^{n}-2$-смежностньй многогранник, соответствуюшие две гиперграни $F_{i}$ и $F_{j}$ имеют непустое пересечение в $P^{n}$. Следовательно, столбцы $i$ и $j$ матришы $\Lambda$ входят в какой-то из ее миноров вида $\Lambda_{(v)}$. Это означает, что определитель этого минора четен и пересечение (28) пусто.

$\S 2.3$. Стабильно комплексные структуры и квазиторические представители в классах кобордизмов. Этот параграф содержит, в основном, результаты, полученные первым автором совместно с Н. Рэем в работах [24]-[26].

Стабильно комплексная структура на (гладком) многообразии $M$ определяется комплексной структурой в векторном расслоении $\tau(M) \oplus \mathbb{R}^{k}$ для некоторого $k$, где $\tau(M)$ - касательное расслоение к $M$, а $\mathbb{R}^{k}$ обозначает тривиальное вешественное $k$-расслоение над $M$. Стабильно комплексное многообразие (в других терминах, 
слабо почти комплексное многообразие или $U$-многообразие) - это многообразие с фиксированной стабильно комплексной структурой, т.е. пара $(M, \xi)$, где $\xi$ - комплексное расслоение, изоморфное, как вешественное расслоение, расслоению $\tau(M) \oplus \mathbb{R}^{k}$ для некоторого $k$. Если $M$ само является комплексным многообразием, то на нем имеется каноническая стабильно комплексная структура $(M, \tau(M))$. Операции несвязного объединения и прямого произведения стабильно комплексных многообразий задают на множестве классов их кобордизмов $[M, \xi]$ структуру градуированного кольца - кольиа комплексных кобордизмов $\Omega^{U}$. Согласно теореме Милнора и Новикова $\Omega^{U} \cong \mathbb{Z}\left[a_{1}, a_{2}, \ldots\right], \operatorname{deg} a_{i}=2 i$ (см. [62], [84]). Это кольцо служит кольцом коэффициентов обобщенной теории (ко)гомологий, известной как комплекснье (ко)бордизмы.

Стабильно комплексным многообразиям было уделено большое внимание в докладе $\Phi$. Хирцебруха на математическом конгрессе в Эдинбурге 1958 г. (см. [60]). Используя гиперповерхности Милнора (см. пример 2.3.10) и теорему Милнора-Новикова, было показано, что каждый класс комплексных кобордизмов содержит неособое алгебраическое многообразие (не обязательно связное). До сих пор остается нерешенной следуюшая проблема.

ПроБЛЕмА 2.3.1 (Хирцебрух). Какие классъц кобордизмов из $\Omega^{U}$ содержат связные неособые алгебраические многообразия?

ПримеР 2.3.2. Группа $\Omega_{2}^{U} \cong \mathbb{Z}$ двумерных кобордизмов порождается классом $\left[\mathbb{C} P^{1}\right]$ (римановой сферы). Каждый класс $k\left[\mathbb{C} P^{1}\right] \in \Omega_{2}^{U}$ содержит неособое алгебраическое многообразие, а именно, при $k>0$ - несвязное объединение $k$ экземпляров $\mathbb{C} P^{1}$, а при $k<0$ - несвязное объединение $k$ экземпляров римановой поверхности рода 2. В то же время связное алгебраическое многообразие содержится лишш в классах кобордизмов вида $k\left[\mathbb{C} P^{1}\right]$, где $k \leqslant 1$.

Задачи выбора образуюших специального вида в кольце $\Omega^{U}$ естественно возникают в теории кобордизмов и ее приложениях. Далее в этом параграфе мы приводим решение аналога проблемы 2.3.1 для квазиторических многообразий, недавно полученное в работах [25] и [26]. Это решение опирается на введение дополнительной структуры на квазиторическом многообразии - полиориентации, задающей на нем каноническую стабильно комплексную структуру в комбинаторных терминах.

Пусть $\pi: M^{2 n} \rightarrow P^{n}$ - квазиторическое многообразие с характеристическим отображением $\ell$. Фиксируя ориентацию тора $T^{n}$ согласно (23), мы получаем, что выбор ориентации для $P^{n}$ эквивалентен выбору ориентации для $M^{2 n}$ (ориентация многогранника $P^{n}$ определяется ориентацией объемлющего пространства $\mathbb{R}^{n}$ ).

ОПРЕДЕЛЕНИЕ 2.3.3. Полиориентацией квазиторического многообразия $M^{2 n}$ называется выбор его ориентации вместе с ориентациями каждого из подмногообразий $M_{i}^{2(n-1)}=\pi^{-1}\left(F_{i}\right), i=1, \ldots, m$.

Таким образом, на данном $M^{2 n}$ всего имеется $2^{m+1}$ полиориентаций.

Полиориентация многообразия $M^{2 n}$ определяет ориентацию каждого нормального расслоения $\nu_{i}:=\nu\left(M_{i} \subset M^{2 n}\right), i=1, \ldots, m$. Так как каждое $\nu_{i}$ является двумерным вещественным расслоением, ориентация $\nu_{i}$ задает в нем структуру одномерного комплексного расслоения. Стационарная подгруппа $T\left(F_{i}\right)$ подмногообразия $M_{i}^{2(n-1)}=\pi^{-1}\left(F_{i}\right)$ действует в нормальном расслоении $\nu_{i}, i=1, \ldots, m$. Таким образом, имеет место следующее утверждение. 
ПРЕДЛОЖЕНИЕ 2.3.4. Въбор полиориентации $M^{2 n}$ эквивалентен выбору ориентации $P^{n}$ вместе с однозначным (а не с точностью до знака) выбором векторов $\boldsymbol{\lambda}_{i}, i=1, \ldots, m$, в (24).

Будем называть характеристическое отображение $\ell$ направленныц, если все окружности $\ell\left(F_{i}\right), i=1, \ldots, m$, ориентированы. Это подразумевает, что знаки векторов гиперграней $\boldsymbol{\lambda}_{i}=\left(\lambda_{1 i}, \ldots, \lambda_{n i}\right)^{t}, i=1, \ldots, m$, выбраны однозначно. В предыдущем параграфемы сводили векторы гиперграней в целочисленную $(n \times m)$-матрицу $\Lambda$. Эта матрица удовлетворяет условию (26). В силу (24) задание матрицы $\Lambda$ эквивалентно заданию направленного характеристического отображения. Пусть $\mathbb{Z}^{\mathscr{F}}$ обозначает $m$-мерный свободньй $\mathbb{Z}$-модуль, порожденный множеством $\mathscr{F}$ гиперграней многогранника $P^{n}$. Тогда матрица $\Lambda$ определяет эпиморфизм $\lambda: \mathbb{Z}^{\mathscr{F}} \rightarrow \mathbb{Z}^{n}\left(\lambda\left(F_{i}\right)=\boldsymbol{\lambda}_{i}\right)$ и эпиморфизм $T^{\mathscr{F}} \rightarrow T^{m}$, который мы будем обозначать той же буквой $\lambda$. Далее мы будем писать $\mathbb{Z}^{m}$ вместо $\mathbb{Z}^{\mathscr{F}}$ и $T^{m}$ вместо $T^{\mathscr{F}}$, используя соглашение, что вектор $e_{i}$ стандартного базиса $\mathbb{Z}^{m}$ соответствует $F_{i} \in \mathbb{Z}^{\mathscr{F}}, i=1, \ldots, m$ (и аналогично для $\left.T^{m}\right)$. Направленная характеристическая пара $\left(P^{n}, \Lambda\right)$ состоит из ориентированного многогранника $P^{n}$ и целочисленной матришы $\Lambda$ (или, эквивалентно, эпиморфизма $\lambda: \mathbb{Z}^{m} \rightarrow \mathbb{Z}^{n}$ ), удовлетворяющей (26).

Предложение 2.3.4 показьвает, что характеристическая пара полиориентированного квазиторического многообразия является направленной. С другой стороны, квазиторическое многообразие, определяемое при помощи конструкции 2.2.2 из направленной характеристической пары, является полиориентированным.

Конструкция 2.3.5. Выбор ориентации в расслоении $\nu_{i}$ над $M_{i}$ задает целочисленный класс Тома в группе когомологий $H^{2}\left(M\left(\nu_{i}\right)\right)$, который представляется некоторым комплексньм одномерным расслоением над пространством Тома $M\left(\nu_{i}\right)$. Отображение Понтрягина-Тома $M^{2 n} \rightarrow M\left(\nu_{i}\right)$ индуцирует некоторое расслоение над $M^{2 n}$, которое мы обозначим $\rho_{i}$. Ограничение $\rho_{i}$ на $M_{i} \subset M^{2 n}$ есть $\nu_{i}$.

Теорема 2.3.6 [26; теорема 3.8]. Каждая полиориентация квазиторического многообразия $M^{2 n}$ задает на нем каноническую стабильно комплексную структуру при помощи следующего изоморфизма вещественных 2 -расслоений:

$$
\tau\left(M^{2 n}\right) \oplus \mathbb{R}^{2(m-n)} \cong \rho_{1} \oplus \cdots \oplus \rho_{m} .
$$

Отсюда следует, что направленная характеристическая пара $\left(P^{n}, \Lambda\right)$ определяет класс кобордизмов $\left[M^{2 n}, \rho_{1} \oplus \cdots \oplus \rho_{m}\right] \in \Omega^{U}$. В то же время, предыдущие конструкции могут быть непосредственно применены к вычислению кольца комплексных кобордизмов $\Omega_{U}^{*}\left(M^{2 n}\right)$ полиориентированного квазиторического многообразия.

Tеорема 2.3 .7 [26; 5.3]. Пусть $v_{i}$ обозначает первый класс Чженя $c_{1}\left(\rho_{i}\right) \in$ $\Omega_{U}^{2}\left(M^{2 n}\right)$ расслоения $\rho_{i}$ в комплексных кобордизмах, $1 \leqslant i \leqslant m$. Тогда кольцо кобордизмов $M^{2 n}$ есть

$$
\Omega_{U}^{*}\left(M^{2 n}\right) \cong \Omega^{U}\left[v_{1}, \ldots, v_{m}\right] /\left(\mathscr{I}_{P}+\mathscr{J}_{\Lambda}\right),
$$

где идеалы $\mathscr{I}_{P}$ и $\mathscr{J}_{\Lambda}$ определяются так же, как и в теореме 2.2.7.

Заметим, что по построению расслоения $\rho_{i}$ класс Чженя $c_{1}\left(\rho_{i}\right)$ двойственен по Пуанкаре вложению $M_{i}^{2(n-1)} \subset M^{2 n}$. В качестве следствия мы получаем замечательный факт, что группы комплексных бордизмов $\Omega_{*}^{U}\left(M^{2 n}\right)$ порождаются вложсенными 
подмногообразиями. Фундаментальный класс кобордизмов $\left\langle M^{2 n}\right\rangle \in \Omega_{U}^{2 n}\left(M^{2 n}\right)$ по определению двойственен по Пуанкаре классу бордизмов точки. Поэтому $\left\langle M^{2 n}\right\rangle=$ $v_{i_{1}} \cdots v_{i_{n}}$ для любого набора $i_{1}, \ldots, i_{n}$ такого, что $F_{i_{1}} \cap \cdots \cap F_{i_{n}}$ - вершина многогранника $P^{n}$.

ПРИМЕР 2.3.8 (многообразие ограниченных флагов [24]). Ограниченнылм флагом в $\mathbb{C}^{n+1}$ называется полньй флаг $U=\left\{U_{1} \subset U_{2} \subset \cdots \subset U_{n+1}=\mathbb{C}^{n+1}\right\}$ такой, что $U_{k}$ содержит координатное подпространство $\mathbb{C}^{k-1}$ (порожденное первыми $k-1$ векторами стандартного базиса) при $2 \leqslant k \leqslant n$. Как показано в [26; пример 2.8], действие тора $T^{n}$ на $\mathbb{C}^{n+1}$ по формуле $t \cdot z=\left(t_{1} z_{1}, \ldots, t_{n} z_{n}, z_{n+1}\right)$ задает на $2 n$-мерном многообразии $B_{n}$ всех ограниченных флагов в $\mathbb{C}^{n+1}$ структуру квазиторического многообразия над кубом $I^{n}$.

ПримеР 2.3.9. В [25] введено семейство многообразий $B_{i, j}, 0 \leqslant i \leqslant j$. Точками многообразия $B_{i, j}$ являются пары $(U, W)$, где $U$ - ограниченньй флаг в $\mathbb{C}^{n+1}$ (см. пример 2.3.8), а $W$ - одномерное подпространство в $U_{1}^{\perp} \oplus \mathbb{C}^{j-i}$. Таким образом, $B_{i, j}$ является пространством гладкого расслоения над $B_{i}$ со слоем $\mathbb{C} P^{j-1}$. В $[26$; пример 2.9] показано, что $B_{i, j}$ является квазиторическим многообразием над произведением $I^{i} \times D^{j-1}$ куба и симплекса.

Канонические стабильно комплексные структуры и полиориентации на многообразиях $B_{n}$ и $B_{i, j}$ описаны в [26; примеры $\left.4.3,4.5\right]$.

ЗАмЕчАниЕ. Произведение двух квазиторических многообразий $M_{1}^{2 n_{1}}$ и $M_{2}^{2 n_{2}}$ над многогранниками $P_{1}^{n_{1}}$ и $P_{2}^{n_{2}}$ является квазиторическим многообразием над $P_{1}^{n_{1}} \times P_{2}^{n_{2}}$. Эта конструкция обобщается на полиориентированные квазиторические многообразия и согласована со стабильно комплексными структурами (детали можно найти в [26; предложение 4.7]).

Как показано в [25], классы многообразий $B_{i, j}$ мультипликативно порождают кольцо $\Omega^{U}$, т.е. каждое $2 n$-мерное стабильно комплексное многообразие содержит в своем классе кобордизмов несвязное объединение многообразий

$$
B_{i_{1}, j_{1}} \times B_{i_{2}, j_{2}} \times \cdots \times B_{i_{k}, j_{k}}
$$

где $\sum_{q=1}^{k}\left(i_{q}+j_{q}\right)-2 k=n$. Каждое такое произведение является квазиторическим многообразием. Это - основной результат работы [25]. Стабильно комплексные структуры на произведениях (29) индуцированы полиориентациями и поэтому сохраняются под действием тора.

ПРИмеР 2.3.10. Стандартный набор многообразий, порождающих кольцо $\Omega^{U}$, состоит из проективных пространств $\mathbb{C} P^{i}, i \geqslant 0$, и гиперповерхностей Милнора $H_{i, j} \subset$ $\mathbb{C} P^{i} \times \mathbb{C} P^{j}, 1 \leqslant i \leqslant j$. Гиперповерхности $H_{i, j}$ определяются следующим образом:

$$
H_{i, j}=\left\{\left(z_{0}: \cdots: z_{i}\right) \times\left(w_{0}: \cdots: w_{j}\right) \in \mathbb{C} P^{i} \times \mathbb{C} P^{j}: \sum_{q=0}^{i} z_{q} w_{q}=0\right\}
$$

При этом, как показано в [25], гиперповерхности $H_{i, j}$ не являются квазиторическими многообразиями при $i \geqslant 2$. 
Для того чтобы задать по-настоящему квазиторических представителей (которые по определению связны) в каждом классе кобордизмов размерности $>2$, остается лиш заменить несвязные объединения произведений (29) на их связные суммы. Это сделано в [26; $\S 6]$, используя конструкцию 1.1 .7 и ее продолжение на полиориентированные квазиторические многообразия.

Teорема 2.3.11 (Бухштабер, Рэй). В размерностях $>2$ каждый класс комnлексных кобордизмов содержит квазиторическое многообразие, непременно связное, стабильно комплексная структура которого индуцирована полиориентацией и, таким образом, согласована с действием тора.

Эта теорема дает решение аналога проблемы 2.3.1 для квазиторических многообразий.

§2.4. Комбинаторные формулы для родов Хирцебруха квазиторических многообразий. Конструкции из предыдущего параграфа открывают дорогу для вычисления инвариантов кобордизмов (чисел Чженя, родов Хирцебруха и др.) полиориентированных квазиторических многообразий в терминах комбинаторики пространства орбит. В данном параграфе мы излагаем результаты, полученные в этом направлении вторым автором в работах [69], [70]. А именно, применяя рассуждения, аналогичные использованным при доказательстве теоремы 1.1.11, мы строим на любом квазиторическом многообразии действие окружности, имеюшее лишь изолированные неподвижные точки. Если $M^{2 n}$ полиориентировано, то такое действие сохраняет стабильно комплексную структуру и его локальные представления вблизи неподвижных точек описываются при помощи характеристической матрицы $\Lambda$. Это позволило вычислить $\chi_{y}$-род Хирцебруха как сумму вкладов, соответствующих вершинам многогранника, в которой слагаемые зависят лишь от "локальной комбинаторики" вблизи вершины. Используя некоторые дополнительные соображения, мы получили в качестве следствия комбинаторные формулы для сигнатуры и рода Тодда многообразия.

ОПРЕДЕЛЕнИЕ 2.4.1. Родом Хирцебруха, ассоциированным со степенным рядом

$$
Q(x)=1+\sum q_{k} x^{k}, \quad q_{k} \in \mathbb{Q},
$$

назьвается кольцевой гомоморфизм $\varphi_{Q}: \Omega^{U} \rightarrow \mathbb{Q}$, который на классе кобордизмов $\left[M^{2 n}\right] \in \Omega_{2 n}^{U}$ принимает значение

$$
\varphi_{Q}\left[M^{2 n}\right]=\left(\prod_{i=1}^{n} Q\left(x_{i}\right),\left\langle M^{2 n}\right\rangle\right) .
$$

Здесь $M^{2 n}$ - гладкое многообразие с комплексной структурой в стабильном касательном расслоении $\tau\left(M^{2 n}\right)$ с полным классом Чженя

$$
c(\tau)=1+c_{1}(\tau)+\cdots+c_{n}(\tau)=\prod_{i=1}^{n}\left(1+x_{i}\right),
$$

а $\left\langle M^{2 n}\right\rangle$ обозначает фундаментальный класс в гомологиях. 
Важным примером рода Хирцебруха является $\chi_{y}$-род, соответствующий ряду

$$
Q(x)=\frac{x\left(1+y e^{-x(1+y)}\right)}{1-e^{-x(1+y)}},
$$

где $y \in \mathbb{R}$ - некоторьй параметр. В частных случаях $y=-1,0,1$ мы получаем соответственно $n$-е число Чженя, род Тодда и сигнатуру многообразия $M^{2 n}$.

В случае комплексного многообразия $M^{2 n}$ значение $\chi_{y}\left(M^{2 n}\right)$ выгисляется в терминах эйлеровых характеристик комплексов Дольбо многообразия $M^{2 n}$. Общую информацию о родах Хирцебруха можно найти в [45].

Далее мы предполагаем заданным полиориентированное квазиторическое многообразие $M^{2 n}$ над некоторым $P^{n}$, с характеристической матрицей $\Lambda$. Это определяет стабильно комплексную структуру на $M^{2 n}$, как описано в предыдущем параграфе. Ориентация $M^{2 n}$ определяет фундаментальньй класс $\left\langle M^{2 n}\right\rangle \in H_{2 n}\left(M^{2 n} ; \mathbb{Z}\right)$.

Конструкция 2.4.2. Пусть $v$ - вершина многогранника, заданная как пересечение $n$ гиперграней:

$$
v=F_{i_{1}} \cap \cdots \cap F_{i_{n}} .
$$

Сопоставим каждой гиперграни $F_{i_{k}}$ ребро $E_{k}:=\bigcap_{j \neq k} F_{i_{k}}$ (т.е. $E_{k}$ содержит $v$ и противоположно $F_{i_{k}}$ ). Пусть $\boldsymbol{e}_{k}-$ вектор вдоль $E_{\boldsymbol{k}}$ с началом $v$. Тогда $\boldsymbol{e}_{1}, \ldots, \boldsymbol{e}_{n}-$ базис $\mathbb{R}^{n}$, который может быть как положительно, так и отрицательно ориентирован, в зависимости от порядка гиперграней в (30). На протяжении всего этого параграфа мы предполагаем, что этот порядок таков, что $\boldsymbol{e}_{1}, \ldots, \boldsymbol{e}_{n}-$ положительно ориентированный базис.

При фиксированном порядке гиперграней в (30) векторы $\boldsymbol{\lambda}_{i_{1}}, \ldots, \boldsymbol{\lambda}_{i_{n}}$ гиперграней в вершине $v$ могут, в свою очередь, составлять как положительно, так и отрицательно ориентированньй базис, в зависимости от знака минора $\Lambda_{(v)}=\left(\boldsymbol{\lambda}_{i_{1}}, \ldots, \boldsymbol{\lambda}_{i_{n}}\right)$ (см. (26)).

ОПРЕДЕЛЕНИЕ 2.4.3. Знак вершины $v=F_{i_{1}} \cap \cdots \cap F_{i_{n}}$ многогранника $P^{n}$ есть

$$
\sigma(v):=\operatorname{det} \Lambda_{(v)} .
$$

Набор знаков вершин многогранника $P^{n}$ является важным инвариантом полиориентированного квазиторического многообразия. Заметим, что обрашение ориентации многообразия $M^{2 n}$ изменяет все знаки $\sigma(v)$ на противоположные. В то же время изменение направления вектора гиперграни обращает знаки лиш тех вершин, которые содержатся в соответствуюшей гиперграни.

Пусть $E$ - ребро $P^{n}$. Стационарная подгруппа 2-мерного подмногообразия $\pi^{-1}(E) \subset M^{2 n}$ является $(n-1)$-мерным тором, которьй мы обозначим $T(E)$. Тогда мы имеем

$$
T(E)=\left\{\left(e^{2 \pi i \varphi_{1}}, \ldots, e^{2 \pi i \varphi_{n}}\right) \in T^{n}: \mu_{1} \varphi_{1}+\cdots+\mu_{n} \varphi_{n}=0\right\}
$$

для некоторых целых чисел $\mu_{1}, \ldots, \mu_{n}$. Мы будем назьвать $\boldsymbol{\mu}:=\left(\mu_{1}, \ldots, \mu_{n}\right)^{t}$ вектором ребра $E$. Этот вектор $\boldsymbol{\mu}$ является примитивньм вектором в двойственной решетке $\left(\mathbb{Z}^{n}\right)^{*}$; он определяется ребром $E$ лишь с точностью до знака. При этом нет канонического способа выбрать эти знаки одновременно для всех ребер. Однако, следующая лемма показывает, что полиориентация многообразия $M^{2 n}$ предоставляет канонический способ выбора знаков векторов ребер “локально” в каждой вершине. 
ЛЕмма 2.4.4. Для каждой вериинъ $v \in P^{n}$ направления векторов $\boldsymbol{\mu}_{1}, \ldots, \boldsymbol{\mu}_{n}$ ребер, сходящихся в $v$, мохсно выбрать таким образом, чтобы $(n \times n)$-матрица $\mathrm{M}_{(v)}:=\left(\boldsymbol{\mu}_{1}, \ldots, \boldsymbol{\mu}_{n}\right)$ удовлетворяла соотношению

$$
\mathrm{M}_{(v)}^{t} \cdot \Lambda_{(v)}=\mathrm{E}
$$

где Е - единичная матрица. Другими словами, $\boldsymbol{\mu}_{1}, \ldots, \boldsymbol{\mu}_{n} u \boldsymbol{\lambda}_{i_{1}}, \ldots, \boldsymbol{\lambda}_{i_{n}}$ являются сопря⿻сенными базисами.

ДокАЗАТЕльство. Вначале выберем знаки векторов ребер в $v$ произвольно и представим $v$ как в $(30)$. Тогда $\boldsymbol{\mu}_{k}$ - вектор ребра $E_{k}$, противоположного гиперграни $F_{i_{k}}$ (конструкция 2.4 .2$), k=1, \ldots, n$. Таким образом, $E_{k} \subset F_{i_{l}}$ и $T\left(F_{i_{l}}\right) \subset T\left(E_{k}\right)$ при $l \neq k$. Следовательно,

$$
\left\langle\boldsymbol{\mu}_{k}, \boldsymbol{\lambda}_{i_{l}}\right\rangle=0, \quad l \neq k
$$

(см. (24) и (31)). Так как $\boldsymbol{\mu}_{k}$ является примитивным вектором, из (32) вытекает, что $\left\langle\boldsymbol{\mu}_{k}, \boldsymbol{\lambda}_{i_{k}}\right\rangle= \pm 1$. Изменяя, если необходимо, знак вектора $\boldsymbol{\mu}_{k}$ на противоположньй, мы получаем

$$
\left\langle\mu_{k}, \lambda_{i_{k}}\right\rangle=1 \text {, }
$$

что вместе с (32) дает $\mathrm{M}_{(v)}^{t} \cdot \Lambda_{(v)}=\mathrm{E}$, что и требовалось.

Далее мы предполагаем, что знаки векторов ребер выбраны в каждой вершине $v$, как описано в лемме 2.4.4. Из этой леммы вытекает также, что векторы $\boldsymbol{\mu}_{1}, \ldots, \boldsymbol{\mu}_{n}$ ребер, сходящихся в $v$, образуют базис решетки $\mathbb{Z}^{n}$ и

$$
\operatorname{det} \mathrm{M}_{(v)}=\sigma(v) .
$$

Пусть $M^{2 n}=M_{P}$ - неособое торическое многообразие, возникаюшее из решеточного простого многогранника $P$, определяемого формулой (1). Тогда $\boldsymbol{\lambda}_{i}=\boldsymbol{l}_{i}$, $i=1, \ldots, m$ (см. пример 2.2.8), в то время как векторы ребер в вершине $v \in P^{n}$ суть примитивные векторы $\boldsymbol{e}_{1}, \ldots, \boldsymbol{e}_{n}$ вдоль ребер с началом в $v$. Как следует из конструкции 2.4.2, в этом случае $\sigma(v)=1$ для всех $v$. Лемма 2.4.4 в этом случае выражает тот факт, что $\boldsymbol{e}_{1}, \ldots, \boldsymbol{e}_{n}$ и $\boldsymbol{l}_{i_{1}}, \ldots, \boldsymbol{l}_{i_{n}}$ - сопряженные базисы решетки $\mathbb{Z}^{n}$. Аналогично, имеет место следующее утверждение.

ПРЕДЛОЖЕНИЕ 2.4.5. Пусть полиориентация квазиторического многообразия $M^{2 n}$ происходит из некоторой $T^{m}$-инвариантной почти комплексной структурь (т.е. комплексной структурь в касательном расслоении $\tau\left(M^{2 n}\right)$ ). Тогда $\sigma(v)=1$ для любой вершины $v \in P^{n}$.

ДоказАТЕльство. Ориентация многогранника $P^{n}$ индуцируется канонической ориентацией почти комплексного многообразия $M^{2 n}$ и ориентацией тора (23). Так как почти комплексная структура на $M^{2 n}$ является $T^{n}$-инвариантной, подмногообразия $M_{i}^{2(n-1)}$, неподвижные относительно $T\left(F_{i}\right) \subset T^{n}$, также являются почти комплексными. Отсюда вытекает, что для любой вершины (30) векторы $\boldsymbol{\lambda}_{i_{1}}, \ldots, \boldsymbol{\lambda}_{i_{n}}$ образуют положительно ориентированный базис пространства $\mathbb{R}^{n}$.

Предложение 2.4.5 дает необходимое условие существования $T^{n}$-инвариантной почти комплексной структуры на $M^{2 n}$ (см. проблему 2.2.10). 
ЗАмЕчАниЕ. Глобально лемма 2.4.4 дает два направления (знака) для вектора ребра, по одному для каждого из его концов. Эти знаки всегда различны при условии, что $M^{2 n}$ - почти комплексное многообразие (например, торическое многообразие), однако в общем случае это неверно.

Пусть $\boldsymbol{\nu}=\left(\nu_{1}, \ldots, \nu_{n}\right)^{t} \in \mathbb{Z}^{n}-$ примитивньй вектор такой, что

$$
\langle\boldsymbol{\mu}, \boldsymbol{\nu}\rangle \neq 0 \text { для любого вектора ребра } \boldsymbol{\mu} \text {. }
$$

Вектор $\boldsymbol{\nu}$ определяет одномерную ориентированную подгруппу

$$
T_{\boldsymbol{\nu}}:=\left\{\left(e^{2 \pi i \nu_{1} \varphi}, \ldots, e^{2 \pi i \nu_{n} \varphi}\right) \in T^{n}: \varphi \in \mathbb{R}\right\}
$$

ЛЕмма 2.4.6 [70; лемма 2.1]. Для любого $\nu$, удовлетворяющего (34), окружность $T_{\nu}$ действует на $M^{2 n}$ с изолированными неподвижсными точками (соответствующими вершинам $\left.P^{n}\right)$. Для каждой вершины $v=F_{i_{1}} \cap \cdots \cap F_{i_{n}}$ действие окружности $T_{\boldsymbol{\nu}}$ индуцирует $S^{1}$-представление в касательном пространстве $T_{v} M^{2 n}$ с весами $\left\langle\boldsymbol{\mu}_{1}, \boldsymbol{\nu}\right\rangle, \ldots,\left\langle\boldsymbol{\mu}_{n}, \boldsymbol{\nu}\right\rangle$.

ЗАмЕчАниЕ. Если $M^{2 n}=M_{P}$ - неособое торическое многообразие, то условие общего положения (34) эквивалентно условию из доказательства теоремы 1.1.11.

ОПРЕДЕЛЕНИЕ 2.4.7. Пусть задан примитивньй вектор $\nu$, удовлетворяющий $(34)$. Введем индекс вершины $v \in P^{n}$ как число отрищательных весов $S^{1}$-представления в $T_{v} M^{2 n}$ из леммы 2.4.6. Таким образом, если $v=F_{i_{1}} \cap \cdots \cap F_{i_{n}}$, то

$$
\operatorname{ind}_{\boldsymbol{\nu}}(v)=\left\{\sharp k:\left\langle\boldsymbol{\mu}_{k}, \boldsymbol{\nu}\right\rangle<0\right\} .
$$

ЗАмЕчАниЕ. Индекс вершины $v$ можно также определить в терминах векторов гиперграней, сходящихся в $v$. Действительно, лемма 2.4.4 показывает, что если $v=F_{i_{1}} \cap \cdots \cap F_{i_{n}}$, то

$$
\boldsymbol{\nu}=\left\langle\boldsymbol{\mu}_{1}, \boldsymbol{\nu}\right\rangle \boldsymbol{\lambda}_{i_{1}}+\cdots+\left\langle\boldsymbol{\mu}_{n}, \boldsymbol{\nu}\right\rangle \boldsymbol{\lambda}_{i_{n}} .
$$

Таким образом, $\operatorname{ind}_{\nu}(v)$ равен числу отрицательных коэффициентов в представлении вектора $\boldsymbol{\nu}$ в виде линейной комбинации базисных векторов $\boldsymbol{\lambda}_{i_{1}}, \ldots, \boldsymbol{\lambda}_{i_{n}}$.

ТЕорема 2.4.8 [70; теорема 3.1]. Для любого $\nu$, удовлетворяющего (34), $\chi_{y}$-род многообразия $M^{2 n}$ может быть вычислен как

$$
\chi_{y}\left(M^{2 n}\right)=\sum_{v \in P^{n}}(-y)^{\operatorname{ind}_{\nu}(v)} \sigma(v) .
$$

Доказательство этой теоремы основано на применении формулы Атьи-Хирцебруха [6] к действию окружности, определенному в лемме 2.4.6.

Значение $\chi_{y}$-рода $\chi_{y}\left(M^{2 n}\right)$ при $y=-1$ равно $n$-му числу Чженя $c_{n}(\xi)\left[M^{2 n}\right]$ для любого $2 n$-мерного стабильно комплексного многообразия $\left[M^{2 n}, \xi\right]$. В случае квазиторического многообразия теорема 2.4.8 дает

$$
c_{n}\left[M^{2 n}\right]=\sum_{v \in P^{n}} \sigma(v) .
$$


Если $M^{2 n}$ является почти комплексным многообразием (например, неособым торическим многообразием), то $\sigma(v)=1$ для всех вершин $v \in P^{n}$ (предложение 2.4.5) и $c_{n}\left[M^{2 n}\right]$ равно эйлеровой характеристике $e\left(M^{2 n}\right)$. Таким образом, для почти комплексных $M^{2 n}$ эйлерова характеристика равна числу вершин многогранника $P^{n}$ (что хорошо известно для торических многообразий). Для обших квазиторических $M^{2 n}$ эйлерова характеристика также равна числу вершин $P^{n}$ (так как эйлерова характеристика произвольного $S^{1}$-многообразия равна сумме эйлеровых характеристик подмногообразий неподвижных точек), но последнее число может быть отлично от $c_{n}\left[M^{2 n}\right]$ (см. пример 2.4.15 ниже).

Значение $\chi_{y}$-рода в $y=1$ есть сигнатура (или $L$-род). Теорема 2.4 .8 в этом случае дает

СлЕДСТВИЕ 2.4.9. Сигнатура полиориентированного квазиторического многообразия $M^{2 n}$ вычисляется как

$$
\operatorname{sign}\left(M^{2 n}\right)=\sum_{v \in P^{n}}(-1)^{\operatorname{ind}_{\nu}(v)} \sigma(v) .
$$

Будучи инвариантом класса ориентированных кобордизмов, сигнатура многообразия $M^{2 n}$ не зависит от стабильно комплексной структуры (т.е. от полиориентации) и определяется лишь ориентацией $M^{2 n}$ (или $P^{n}$ ). Следующая модификация следствия 2.4.9 дает формулу для $\operatorname{sign}\left(M^{2 n}\right)$, которая не зависит от полиориентации.

СлЕДСТвИЕ 2.4.10 [70; следствие 3.3]. Сигнатура ориентированного квазиторического многообразия $M^{2 n}$ вычисляется как

$$
\operatorname{sign}\left(M^{2 n}\right)=\sum_{v \in P^{n}} \operatorname{det}\left(\widetilde{\boldsymbol{\mu}}_{1}, \ldots, \widetilde{\boldsymbol{\mu}}_{n}\right),
$$

где $\widetilde{\boldsymbol{\mu}}_{k}, k=1, \ldots, n,-$ векторы ребер, сходящихся в $v$, направленныее таким образом, что $\left\langle\widetilde{\boldsymbol{\mu}}_{k}, \boldsymbol{\nu}\right\rangle>0$.

Если $M^{2 n}=M_{P}$ является неособым торическим многообразием, то $\sigma(v)=1$ для любой вершины $v \in P^{n}$ и следствие 2.4 .9 дает

$$
\operatorname{sign}\left(M_{P}\right)=\sum_{v \in P^{n}}(-1)^{\operatorname{ind}_{\nu}(v)} .
$$

Так как в этом случае индекс $\operatorname{ind}_{\nu}(v)$ совпадает с индексом из доказательства теоремы 1.1.11, мы получаем

$$
\operatorname{sign}\left(M_{P}\right)=\sum_{k=0}^{n}(-1)^{k} h_{k}(P)
$$

Заметим, что если $n$ нечетно, то правая часть предыдушей формулы обрашается в нуль ввиду соотношений Дена-Соммервилля. Формула (36) появляется в более общем контексте в недавней работе Леунга и Райнера [55]. Число в правой части формулы (36) возникает в следующей известной комбинаторной гипотезе. 
ПроблемА 2.4.11 (гипотеза Черни-Дэвиса). Пусть $K-(2 q-1)$-мерньй флаговый горенштейнов ${ }^{*}$ комплекс с $h$-вектором $\left(h_{0}, h_{1}, \ldots, h_{2 q-1}\right)$. Верно ли, что

$$
(-1)^{q}\left(h_{0}-h_{1}+\cdots+h_{2 q}\right) \geqslant 0 ?
$$

Эта гипотеза была высказана в [29; гипотеза D] для флаговых симплициальных гомологических сфер. Ее обобщение на случай горенштейновых ${ }^{*}$ комплексов принадлежит Стенли [83; гипотеза 4]. Гипотеза Черни-Дэвиса тесно связана со следующей гипотезой из дифференциальной геометрии.

ПроБЛЕма 2.4.12 (гипотеза Хопфа). Пусть $M^{2 q}$ - риманово многообразие неположительной секиионной кривизнь. Верно ли, что эйлерова характеристика $\chi\left(M^{2 n}\right)$ удовлетворяет неравенству

$$
(-1)^{q} \chi\left(M^{2 q}\right) \geqslant 0 ?
$$

Известно, что обе гипотезы имеют место при $q=1,2$, а также в ряде частных случаев. Дополнительные подробности можно найти в [29]. Взаимосвязям двух предыдущих проблем с сигнатурой торического многообразия посвяшена работа [55].

Теперь мы вернемся снова к $\chi_{y}$-роду полиориентированного квазиторического многообразия. Следуюшим важным частным случаем является род Тодда, соответствующий $y=0$. В этом случае слагаемые в формуле из теоремы 2.4.8 не определены для вершин, имеюших индекс 0 , так что этот случай требует дополнительного анализа.

Теорема 2.4.13 [69; теорема 7], [70; теорема 3.4]. Род Тодда полиориентированного квазиторического многообразия вычисляется как

$$
\operatorname{td}\left(M^{2 n}\right)=\sum_{v \in P^{n}: \operatorname{ind}_{\nu}(v)=0} \sigma(v)
$$

(сумма берется по всем вершинам индекса 0).

В случае неособого торического многообразия имеется лишь одна вершина индекса 0. Это - “нижняя" вершина многогранника $P^{n}$, для которой все инцидентные ребра направлены от нее (в обозначениях, используемых при доказательстве теоремы 1.1.11). Так как $\sigma(v)=1$ для любой $v \in P^{n}$, теорема 2.4.13 дает $\operatorname{td}\left(M_{P}\right)=1$, что хорошо известно (см., например, [40; §5.3]; род Тодда для алгебраических многообразий равен арифметическому роду).

Если же $M^{2 n}$ является почти комплексным многообразием, то, как показьвают предложение 2.4.5 и теорема 2.4.13, мы имеем $\operatorname{td}\left(M^{2 n}\right)>0$.

ПримеР 2.4.14. Проективное пространство $\mathbb{C} P^{2}$, рассматриваемое как торическое многообразие, возникает из стандартного решеточного 2-симплекса $\Delta^{2}$ (с вершинами $\left.v_{1}=(0,0), v_{2}=(1,0), v_{3}=(0,1)\right)$. Ориентация стандартная (определяется комплексной структурой). Полиориентация определяется векторами гиперграней $\boldsymbol{\lambda}_{1}, \boldsymbol{\lambda}_{2}, \boldsymbol{\lambda}_{3}$, которые в этом случае являются примитивными нормальными векторами, направленными внутрь многогранника. Векторы ребер являются примитивными векторами вдоль ребер, направленными от вершины. Это изображено на рис. 5. Соответствуюшая стабильно комплексная структура стандартна, т.е. определяется 
изоморфизмом расслоений $\tau\left(\mathbb{C} P^{2}\right) \oplus \mathbb{R}^{2} \cong \bar{\eta} \oplus \bar{\eta} \oplus \bar{\eta}$, где $\eta$ - каноническое одномерное расслоение Хопфа. Вычислим род Тодда и сигнатуру при помощи следствия 2.4.9 и теоремы 2.4.13. Мы имеем $\sigma\left(v_{1}\right)=\sigma\left(v_{2}\right)=\sigma\left(v_{3}\right)=1$. Возьмем $\boldsymbol{\nu}=(1,2)$, тогда $\operatorname{ind}_{\nu}\left(v_{1}\right)=0, \operatorname{ind}_{\nu}\left(v_{2}\right)=1, \operatorname{ind}_{\nu}\left(v_{3}\right)=2$ (напомним, что индекс есть число отрищательных скалярных произведений векторов ребер с $\boldsymbol{\nu})$. Итак, $\operatorname{sign}\left(\mathbb{C} P^{2}\right)=$ $\operatorname{sign}\left[\mathbb{C} P^{2}, \bar{\eta} \oplus \bar{\eta} \oplus \bar{\eta}\right]=1, \operatorname{td}\left(\mathbb{C} P^{2}\right)=\operatorname{td}\left[\mathbb{C} P^{2}, \bar{\eta} \oplus \bar{\eta} \oplus \bar{\eta}\right]=1$.

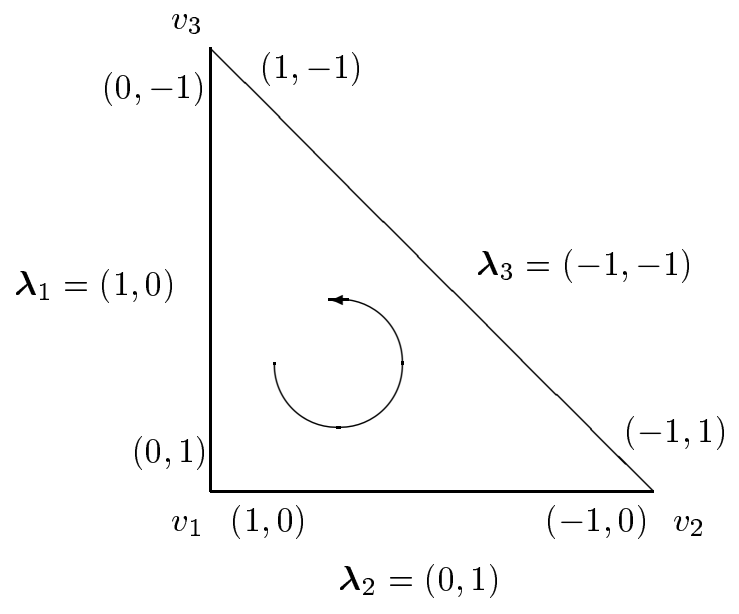

Рис. 5. $\tau\left(\mathbb{C} P^{2}\right) \oplus \mathbb{C} \simeq \bar{\eta} \oplus \bar{\eta} \oplus \bar{\eta}$

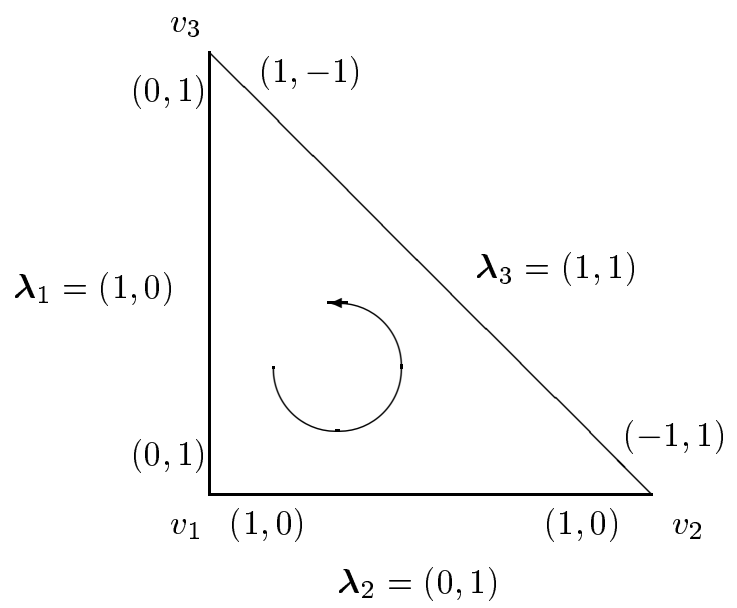

Рис. 6. $\tau\left(\mathbb{C} P^{2}\right) \oplus \mathbb{C} \simeq \bar{\eta} \oplus \bar{\eta} \oplus \eta$

ПримеР 2.4.15. Теперь рассмотрим $\mathbb{C} P^{2}$ с полиориентацией, определяемой тремя векторами гиперграней $\boldsymbol{\lambda}_{1}, \boldsymbol{\lambda}_{2}, \boldsymbol{\lambda}_{3}$, изображенными на рис. 6 . Эта полиориентация отличается от предыдущего примера знаком вектора $\boldsymbol{\lambda}_{3}$. Соответствующая стабильно комплексная структура определяется изоморфизмом $\tau\left(\mathbb{C} P^{2}\right) \oplus \mathbb{R}^{2} \cong \bar{\eta} \oplus \bar{\eta} \oplus \eta$. 
Используя (33), вычисляем

$$
\sigma\left(v_{1}\right)=\left|\begin{array}{ll}
1 & 0 \\
0 & 1
\end{array}\right|=1, \quad \sigma\left(v_{2}\right)=\left|\begin{array}{cc}
-1 & 1 \\
1 & 0
\end{array}\right|=-1, \quad \sigma\left(v_{3}\right)=\left|\begin{array}{cc}
0 & 1 \\
1 & -1
\end{array}\right|=-1 .
$$

Для $\boldsymbol{\nu}=(1,2)$ находим $\operatorname{ind}_{\nu}\left(v_{1}\right)=0, \operatorname{ind}_{\nu}\left(v_{2}\right)=0, \operatorname{ind}_{\nu}\left(v_{3}\right)=1$. Итак, $\operatorname{sign}\left[\mathbb{C} P^{2}\right.$, $\bar{\eta} \oplus \bar{\eta} \oplus \eta]=1, \operatorname{td}\left[\mathbb{C} P^{2}, \bar{\eta} \oplus \bar{\eta} \oplus \eta\right]=0$. Заметим, что в этом случае формула (35) дает $c_{n}\left[\mathbb{C} P^{2}, \bar{\eta} \oplus \bar{\eta} \oplus \eta\right]=\sigma\left(v_{1}\right)+\sigma\left(v_{2}\right)+\sigma\left(v_{3}\right)=-1$, в то время как эйлерова характеристика $\mathbb{C} P^{2}$ есть $c_{n}\left[\mathbb{C} P^{2}, \bar{\eta} \oplus \bar{\eta} \oplus \bar{\eta}\right]=3$.

$T^{n}$-эквивариантные стабильно комплексные и почти комплексные многообразия рассматриваются в работах Хаттори [44] и Масуды [57] как альтернативное обобшение торических многообразий (соответствующие многообразия названы в этих работах унитарными торическими многообразиями). Вместо характеристических отображений из [33] для описания комбинаторной структуры пространства орбит в [57] используется понятие мульти-веера. Мульти-веер представляет собой набор конусов, которые, в отличие от обычного веера, могут накладьваться друг на друга. Род Тодда унитарного торического многообразия был вычислен в [57] в терминах конусов мульти-веера. Ответ эквивалентен нашей теореме 2.4.13 в случае квазиторических многообразий.

$\S 2.5$. Задача классификации квазиторических многообразий над заданным простым многогранником. Строго говоря, имеется две задачи классификации: эквивариантная (с точностью до эквивариантного диффеоморфизма) и топологическая (с точностью до диффеоморфизма). В силу предложения 2.2.3 задача эквивариантной классификации сводится к описанию всех характеристических отображений для данного простого многогранника $P^{n}$. Задача топологической классификации, как правило, требует дополнительного анализа.

Пусть $M^{2 n}$ - некоторое квазиторическое многообразие над $P^{n}$ с характеристическим отображением $\ell$. Мы также будем предполагать, что первые $n$ гиперграней $F_{1}, \ldots, F_{n}$ имеют общую вершину.

ЛЕмма 2.5.1. С точностью до $\psi$-преобразования, мы можем предполагать, что $\ell\left(F_{i}\right)$ есть $i$-я координатная подгруппа $T_{i} \subset T^{n}, i=1, \ldots, n$.

ДокАЗАТЕЛьство. Так как одномерные подгруппы $\ell\left(F_{i}\right), i=1, \ldots, n$, порождают $T^{n}$, мы можем определить $\psi$ как произвольный автоморфизм тора $T^{n}$, которьй переводит $\ell\left(F_{i}\right)$ в $T_{i}, i=1, \ldots, n$.

Отсюда также вытекает, что на $M^{2 n}$ можно выбрать полиориентацию таким образом, что соответствуюшая характеристическая $(n \times m)$-матрища $\Lambda$ имеет вид $(\mathbb{E} \mid *)$, где $\mathbb{E}$ - единичная матрица, а * означает некоторую целочисленную $(n \times(m-n))$-матрищу.

В простейшем случае $P^{n}=\Delta^{n}$ эквивариантная (и топологическая) классификация квазиторических многообразий сводится к следующему простому результату.

ПРЕДЛОЖЕНИЕ 2.5.2. Любое квазиторическое многообразие над симплексом $\Delta^{n}$ әквивариантно диффеоморфно $\mathbb{C} P^{n}$ (рассматриваемому как торическое многообразие, см. примеры 2.1 .5 и 2.2.8). 
ДокАЗАТЕльСтво. Характеристическое отображение для $\mathbb{C} P^{n}$ имеет вид

$$
\ell_{\mathbb{C} P^{n}}\left(F_{i}\right)=T_{i}, \quad i=1, \ldots, n, \quad \ell_{\mathbb{C} P^{n}}\left(F_{n+1}\right)=S_{d},
$$

где $S_{d}^{1}:=\left\{\left(e^{2 \pi i \varphi}, \ldots, e^{2 \pi i \varphi}\right) \in T^{n}\right\}, \varphi \in \mathbb{R},-$ диагональная подгруппа в $T^{n}$. Пусть $M^{2 n}$ - квазиторическое многообразие над $\Delta^{n}$ с характеристическим отображением $\ell_{M}$. В силу леммы 2.5.1 мы можем предполагать, что $\ell\left(F_{i}\right)=T_{i}, i=1, \ldots, n$. Тогда из условия (26) легко выводится

$$
\ell\left(F_{n+1}\right)=\left\{\left(e^{2 \pi i \varepsilon_{1} \varphi}, \ldots, e^{2 \pi i \varepsilon_{n} \varphi}\right) \in T^{n}\right\}, \quad \varphi \in \mathbb{R},
$$

где $\varepsilon_{i}= \pm 1, i=1, \ldots, n$. Теперь определим автоморфизм $\psi: T^{n} \rightarrow T^{n}$ как

$$
\psi\left(e^{2 \pi i \varphi_{1}}, \ldots, e^{2 \pi i \varphi_{n}}\right)=\left(e^{2 \pi i \varepsilon_{1} \varphi_{1}}, \ldots, e^{2 \pi i \varepsilon_{n} \varphi_{n}}\right) .
$$

Тогда легко видеть, что $\psi \cdot \ell_{M}=\ell_{\mathbb{C} P^{n}}$, и утверждение вытекает из предложения 2.2.3.

Заметим, что эквивариантньй диффеоморфизм, существование которого утверждается в предложении 2.5.2, вообще говоря, не сохраняет ориентацию квазиторического многообразия $M^{2 n}$.

Следующим случаем, когда задача топологической и эквивариантной классификации допускает полное решение, является случай $n=2$ (т.е. случай квазиторических многообразий над многоугольниками).

ПримеР 2.5.3. Поверхностью Хириебруха назьвается двумерное комплексное многообразие $H_{p}=\mathbb{C} P\left(\zeta_{p} \oplus \mathbb{C}\right)$, где $\zeta_{p}$ - одномерное комплексное расслоение над $\mathbb{C} P^{1}$ с первым классом Чженя $p$ и $\mathbb{C}$ - одномерное тривиальное расслоение, а $\mathbb{C} P(\cdot)$ обозначает проективизацию комплексного векторного расслоения. Таким образом, имеется расслоение $H_{p} \rightarrow \mathbb{C} P^{1}$ со слоем $\mathbb{C} P^{1}$. Топологически $H_{p}$ представляет собой $S^{2} \times S^{2}$ при четных $p$ и $\mathbb{C} P^{2} \# \overline{\mathbb{C P}}^{2}$ при нечетных $p$, где $\overline{\mathbb{C P}}^{2}$ обозначает $\mathbb{C} P^{2}$ с обрашенной ориентацией. Как показано в [40; с. 8], поверхности Хирцебруха являются неособьми проективными торическими многообразиями. Пространство орбит для $H_{p}$, рассматриваемого как квазиторическое многообразие, представляет собой комбинаторный квадрат; для нахождения соответствуюших характеристических отображений можно воспользоваться примером 2.2.8 (см. также [33; пример 1.19]).

Теорема 2.5 .4 [64; с. 553]. Любое квазиторическое многообразие размерности 4 эквивариантно диффеоморфно эквивариантной связной сумме нескольких экземпляров $\mathbb{C} P^{2}$ и поверхностей Хирчебруха $H_{p}$.

СлЕДСТВИЕ 2.5.5. Любое квазиторическое многообразие размерности 4 диффеоморфно связной сумме нескольких әкземпляров $\mathbb{C P}^{2}, \overline{\mathbb{C}}^{2}$ и $S^{2} \times S^{2}$.

Задача классификации квазиторических многообразий надзаданньм простым многогранником может рассматриваться как обобшение соответствующей задачи для неособых торических многообразий. В [63] каждому торическому многообразию над простым 3-многогранником $P^{3}$ ставится в соответствие два целых веса на каждом ребре двойственного симплициального комплекса $K_{P}$. При помоши специальных "условий монодромии" на веса в [63] построена полная классификация торических многообразий над простыми 3-многогранниками с числом гиперграней не более 8. В [53] 
аналогичная конструкция использована для получения классификации торических многообразий над $P^{n}$ с числом гиперграней $m=n+2$ (заметим, что любой такой простой многогранник является произведением двух симплексов).

В работе [36] конструкция весов из [63] обобщена на случай квазиторических многообразий. Это позволило получить критерий [36; теорема 3] существования квазиторического многообразия с данным набором весов и знаков вершин $\sigma(v)$ (см. определение 2.4.3; заметим, что наши знаки вершин соответствуют двухцветной раскраске, используемой в [36]). В качестве приложения в [36] получена полная классификация характеристических отображений для куба $I^{3}$ и ряд результатов относительно классификации квазиторических многообразий над произведениями произвольного числа симплексов.

\section{Глава 3. Момент-угол комплексы}

§.1. Момент-угол многообразия $\mathscr{Z}_{P}$, определяемые простыми многогранниками. Для любого простого многогранника $P^{n}$ с $m$ гипергранями Дэвис и Янушкевич ввели в [33] пространство $\mathscr{Z}_{P}$ с действием тора $T^{m}$ с пространством орбит $P^{n}$. Это пространство является универсальным для всех квазиторических многообразий над $P^{n}$ в том смысле, что для любого квазиторического многообразия $\pi: M^{2 n} \rightarrow P^{n}$ сушествует главное $T^{m-n}$-расслоение $\mathscr{Z}_{P} \rightarrow M^{2 n}$, композиция которого с проекцией $\pi$ дает проекцию на пространство орбит для $\mathscr{Z}_{P}$. Пространство $\mathscr{Z}_{P}$ и его обобшения оказались очень важным и эффективным инструментом для изучения различных комбинаторных объектов, таких как кольца Стенли-Райснера, конфигурации подпространств, кубические комплексы и т. д., методами алгебраической топологии. В этом параграфе мы воспроизводим оригинальное определение $\mathscr{Z}_{P}$ и модифищируем его для последуюших обобщений.

Пусть $\mathscr{F}=\left\{F_{1}, \ldots, F_{m}\right\}-$ множество гиперграней многогранника $P^{n}$. Для каждой гиперграни $F_{i} \in \mathscr{F}$ обозначим через $T_{F_{i}}$ одномерную координатную подгруппу тора $T^{\mathscr{F}} \cong T^{m}$, соответствующую $F_{i}$. Поставим теперь в соответствие каждой грани $G$ координатную торическую подгруппу

$$
T_{G}=\bigoplus_{F_{i} \supset G} T_{F_{i}} \subset T^{\mathscr{F}} .
$$

Заметим, что $\operatorname{dim} T_{G}=\operatorname{codim} G$. Напомним, что для каждой точки $q \in P^{n}$ мы обозначали через $G(q)$ единственную грань, содержашую точку $q$ в своей внутренности.

ОПРЕДЕЛЕНИЕ 3.1 .1 [33; $\S$ 4]. Для любого простого многогранника $P^{n}$ введем фактор-пространство

$$
\mathscr{Z}_{P}=\left(T^{\mathscr{F}} \times P^{n}\right) / \sim
$$

где $\left(t_{1}, q\right) \sim\left(t_{2}, q\right)$ тогда и только тогда, когда $t_{1} t_{2}^{-1} \in T_{G(q)}$.

Свободное действие тора $T^{m}$ на $T^{\mathscr{F}} \times P^{n}$ определяет действие на $\mathscr{Z}_{P}$ с пространством орбит $P^{n}$. Пусть $\rho: \mathscr{Z}_{P} \rightarrow P^{n}-$ проекция на пространство орбит. Действие $T^{m}$ на $\mathscr{Z}_{P}$ является свободным над внутренностью многогранника, в то время как каждая вершина $v \in P^{n}$ представляет орбиту $\rho^{-1}(v)$ с максимальной стационарной подгруппой размерности $n$. 
ЛЕмма 3.1.2. Пространство $\mathscr{Z}_{P}$ является гладким многообразием размерности $m+n$.

В настоящей работе мы предоставляем несколько различных способов доказательства этой леммы, каждый из которых возникает из эквивалентного определения $\mathscr{Z}_{P}$. Для того чтобы привести первое доказательство, нам понадобится следуюшее простой топологический факт.

ПрЕДЛОЖЕнИЕ 3.1.3. Тор $T^{k}$ допускает гладкое вложсение в $\mathbb{R}^{k+1}$.

ДокАЗАТЕЛЬСТво. Мы построим вложение при помощи гладкой функции $g_{k+1}\left(x_{1}, \ldots, x_{k+1}\right)$ такой, что уравнение $g_{k+1}=0$ определяет гиперповерхность, диффеоморфную $T^{k}$. При $k=1$ возьмем $g_{2}\left(x_{1}, x_{2}\right)=x_{1}^{2}+\left(x_{2}-2\right)^{2}-1$; далее продолжим по индукции по $k$. Пусть мы имеем функцию $g_{i}\left(x_{1}, \ldots, x_{i}\right)$ такую, что $\left\{g_{i}=0\right\}$ определяет гладкое вложение $T^{i-1} \hookrightarrow \mathbb{R}^{i}$, причем $x_{i}>0$ для любого набора $\left(x_{1}, \ldots, x_{i}\right)$, удовлетворяющего уравнению $g_{i}=0$. Тогда положим

$$
g_{i+1}\left(x_{1}, \ldots, x_{i}, x_{i+1}\right):=g_{i}\left(x_{1}, \ldots, x_{i-1}, \sqrt{x_{i}^{2}+x_{i+1}^{2}}\right) .
$$

Нетрудно проверить, что гиперповерхность $\left\{g_{i+1}=0\right\} \subset \mathbb{R}^{i+1}$ диффеоморфна $T^{i}$.

ДокАЗАТЕЛЬСТво ЛЕмМы 3.1.2. Конструкция 2.1.6 определяет атлас $\left\{U_{v}\right\}$ для $P^{n}$ как многообразия с углами. Множество $U_{v}$ содержит вершину $v$ и диффеоморфно $\mathbb{R}_{+}^{n}$. Тогда $\rho^{-1}\left(U_{v}\right) \cong T^{m-n} \times \mathbb{R}^{2 n}$. Мы утверждаем, что $T^{m-n} \times \mathbb{R}^{2 n}$ может быть реализовано как открытое подмножество в $\mathbb{R}^{m+n}$, таким образом задавая карту для $\mathscr{Z}_{P}$. Чтобы увидеть это, вложим $T^{m-n}$ в $\mathbb{R}^{m-n+1}$ как замкнутую гиперповерхность $H$ (предложение 3.1.3). Так как нормальное расслоение тривиально, малая окрестность гиперповерхности $H \subset \mathbb{R}^{m-n+1}$ гомеоморфна $T^{m-n} \times \mathbb{R}$. Беря декартово произведение с $\mathbb{R}^{2 n-1}$, мы получаем открытое подмножество в $\mathbb{R}^{m+n}$, гомеоморфное $T^{m-n} \times \mathbb{R}^{2 n}$.

Следующее утверждение легко вытекает из определения $\mathscr{Z}_{P}$.

ПРЕДЛОЖЕНИЕ 3.1.4. Если $P=P_{1} \times P_{2}$ для некоторых простых многогранников $P_{1}, P_{2}$, то $\mathscr{Z}_{P}=\mathscr{Z}_{P_{1}} \times \mathscr{Z}_{P_{2}}$. Если $G \subset P-$ грань, то $\mathscr{Z}_{G}$ является подмногообразием в $\mathscr{Z}_{P}$.

Предположим теперь, что нам дано характеристическое отображение $\ell$ на $P^{n}$ и $M^{2 n}(\ell)$ - определяемое им квазиторическое многообразие (конструкция 2.2.2). Выбирая полиориентацию произвольньм образом, мы получаем направленное характеристическое отображение $\lambda: T^{\mathscr{F}} \rightarrow T^{n}$. Обозначим его ядро через $H(\ell)$ (оно зависит лишш от $\ell)$; тогда $H(\ell)$ есть $(m-n)$-мерная торическая подгруппа в $T^{\mathscr{F}}$.

ПРЕДЛОЖЕНИЕ 3.1.5. Подгруппа $H(\ell)$ действует на $\mathscr{Z}_{P}$ свободно, таким образом определяя главное $T^{m-n}$-расслоение $\mathscr{Z}_{P} \rightarrow M^{2 n}(\ell)$.

ДокАЗАТЕльство. Как вытекает из (26), подгруппа $H(\ell)$ пересекает каждую стационарную подгруппу лишь по единище. Это означает, что действие $H(\ell)$ на $\mathscr{Z}_{P}$ свободно. По определению $\mathscr{Z}_{P}$ и $M^{2 n}(\ell)$ проекция $\lambda \times \mathrm{id}: T^{\mathscr{F}} \times P^{n} \rightarrow T^{n} \times P^{n}$ определяет проекцию

$$
\left(T^{\mathscr{F}} \times P^{n}\right) / \sim \longrightarrow\left(T^{n} \times P^{n}\right) / \sim,
$$

которая описывает $\mathscr{Z}_{P}$ как главное $T^{m-n}$-расслоение над $M^{2 n}(\ell)$. 
Для упрошения обозначений мы будем писать $T^{m}, \mathbb{C}^{m}$ и т. д. вместо $T^{\mathscr{F}}, \mathbb{C}^{\mathscr{F}}$ и т. д. Определим единичный полидиск $\left(D^{2}\right)^{m}$ в комплексном пространстве как

$$
\left(D^{2}\right)^{m}=\left\{\left(z_{1}, \ldots, z_{m}\right) \in \mathbb{C}^{m}:\left|z_{i}\right| \leqslant 1, i=1, \ldots, m\right\}
$$

Тогда $\left(D^{2}\right)^{m}$ инвариантен относительно стандартного действия $T^{m}$ на $\mathbb{C}^{m}$, а пространство орбит есть стандартньй куб $I^{m} \subset \mathbb{R}_{+}^{m}$.

Лемма 3.1.6. Кубическое вложение $i_{P}: P^{n} \rightarrow I^{m}$ из конструкиии 1.5 .5 накрывается әквивариантным вложением $i_{e}: \mathscr{Z}_{P} \rightarrow\left(D^{2}\right)^{m}$.

ДокАЗАТЕльство. Напомним, что кубическое разбиение многогранника $P^{n}$ состоит из кубов $C_{v}^{n}$, соответствуюших вершинам $v \in P^{n}$. Заметим, что $C_{v}^{n}$ содержится в открытом множестве $U_{v} \subset P^{n}$ (см. конструкцию 2.1.6). Вложение $C_{v}^{n} \subset U_{v}$ накрывается эквивариантным вложением $B_{v} \subset \mathbb{C}^{m}$, где $B_{v}=\rho^{-1}\left(C_{v}^{n}\right)$ - замкнутое подмножество, гомеоморфное $\left(D^{2}\right)^{n} \times T^{m-n}$. Так как $\mathscr{Z}_{P}=\bigcup_{v \in P^{n}} B_{v}$ и $B_{v}$ инвариантно относительно $T^{m}$-действия, получаемое вложение $\mathscr{Z}_{P} \rightarrow\left(D^{2}\right)^{m}$ эквивариантно.

Из доказательства вытекает, что многообразие $\mathscr{Z}_{P}$ представляется в виде объединения $f_{n-1}(P)$ замкнутых $T^{m}$-инвариантных карт $B_{v}$. В $\S 3.3$ мы приводим два различных способа построения клеточного разбиения каждого из $B_{v}$, таким образом вводя на $\mathscr{Z}_{P}$ структуру клеточного комплекса. Пока мы лишь отметим, что если $v=F_{i_{1}} \cap \cdots \cap F_{i_{n}}$, то

$$
i_{e}\left(B_{v}\right)=\left(D^{2}\right)_{i_{1}, \ldots, i_{n}}^{n} \times T_{[m] \backslash\left\{i_{1}, \ldots, i_{n}\right\}}^{m-n} \subset\left(D^{2}\right)^{m},
$$

или, точнее,

$$
i_{e}\left(B_{v}\right)=\left\{\left(z_{1}, \ldots, z_{m}\right) \in\left(D^{2}\right)^{m}:\left|z_{i}\right|=1 \text { при } i \notin\left\{i_{1}, \ldots, i_{n}\right\}\right\} .
$$

Вспоминая, что вершины $P^{n}$ соответствуют максимальным симплексам многогранной сферы $K_{P}$ (границы полярного многогранника $P^{*}$ ), мы можем написать

$$
i_{e}\left(\mathscr{Z}_{P}\right)=\bigcup_{I \in K_{P}}\left(D^{2}\right)_{I} \times T_{[m] \backslash I} \subset\left(D^{2}\right)^{m} .
$$

Это может рассматриваться как альтернативное определение $\mathscr{Z}_{P}$. Вводя полярные координаты на $\left(D^{2}\right)^{m}$, мы видим, что $i_{e}\left(B_{v}\right)$ параметризуется $n$ радиальными (или моментньми) и $m$ угловыми координатами. Ввиду этого мы называем $\mathscr{Z}_{P}$ моментугол многообразием, определяемьм многогранником $P^{n}$.

ПримеР 3.1.7. Пусть $P^{n}=\Delta^{n}$ (n-симплекс). Тогда $\mathscr{Z}_{P}$ гомеоморфио $(2 n+1)$-сфеpe $S^{2 n+1}$. Кубический комплекс $\mathscr{C}\left(\Delta^{n}\right)$ (см. конструкцию 1.5.5) состоит из $(n+1)$ кубов $C_{v}^{n}$. Каждое подмножество $B_{v}=\rho^{-1}\left(C_{v}^{n}\right)$ гомеоморфно $\left(D^{2}\right)^{n} \times S^{1}$. В частности, при $n=1$ мы получаем известное представление 3 -сферы $S^{3}$ в виде объединения двух полноторий $D^{2} \times S^{1}$.

Другой способ построения эквивариантного вложения $\mathscr{Z}_{P}$ в $\mathbb{C}^{m}$ основан на использовании конструкции 1.1.4. 
Конструкция 3.1.8. Формула (6) определяет (аффинное) вложение $A_{P}: P^{n} \hookrightarrow$ $\mathbb{R}_{+}^{m}$. Это вложение накрывается эквивариантным вложением $\mathscr{Z}_{P} \hookrightarrow \mathbb{C}^{m}$. Выбор матрищы $W$ в конструкции 1.1.4 задает базис в $(m-n)$-мерном подпространстве, ортогональном $n$-мерной плоскости, содержашей $A_{P}\left(P^{n}\right)$ (см. (6)). Отсюда вытекает следуюшее утверждение.

СлЕДСТвИЕ 3.1 .9 (см. также [26; §3]). Вложение $\mathscr{Z}_{P} \hookrightarrow \mathbb{C}^{m}$ имеет тривиальное нормальное расслоение.

$\S$ 3.2. Общие момент-угол комплексы $\mathscr{Z}_{K}$. В этом параграфе мы обобщаем конструкцию пространства $\mathscr{Z}_{P}$ на случай произвольного симплициального комплекса $K$. Получаемое пространство $\mathscr{Z}_{K}$ в общем случае уже не является многообразием, однако это имеет место в случае, когда $K$ является симплициальной сферой.

Обозначим через $\rho$ каноническую проекцию $\left(D^{2}\right)^{m} \rightarrow I^{m}$, а также ее ограничение на произвольное замкнутое $T^{m}$-инвариантное подмножество полидиска $\left(D^{2}\right)^{m}$. Для каждой грани $C_{I \subset J}$ куба $I^{m}$ (см. (19)) определим

$$
\begin{aligned}
B_{I \subset J} & :=\rho^{-1}\left(C_{I \subset J}\right) \\
& =\left\{\left(z_{1}, \ldots, z_{m}\right) \in\left(D^{2}\right)^{m}: z_{i}=0 \text { при } i \in I,\left|z_{i}\right|=1 \text { при } i \notin J\right\} .
\end{aligned}
$$

Если \#I=i,\#J=j, то $B_{I \subset J} \cong\left(D^{2}\right)^{j-i} \times T^{m-j}$, где сомножители-диски $D^{2} \subset$ $\left(D^{2}\right)^{j-i}$ параметризуются множеством $J \backslash I$, а сомножители-окружности $S^{1} \subset T^{m-j}$ параметризуются множеством $[m] \backslash J$.

ОПРЕДЕЛЕНИЕ 3.2.1. Пусть $\mathscr{C}$ - кубический подкомплекс в $I^{m}$. Момент-угол комплексом та $(\mathscr{C})$, соответствуюшим $\mathscr{C}$, назьвается $T^{m}$-инвариантное разбиение подмножества $\rho^{-1}(\mathscr{C})$ на "момент-угол" блоки $B_{I \subset J}(38)$, соответствующие граням $C_{I \subset J}$ кубического комплекса $\mathscr{C}$. Таким образом, та $(\mathscr{C})$ определяется из коммутативной диаграммы

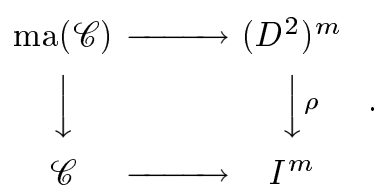

Тор $T^{m}$ действует на та $(\mathscr{C})$ с пространством орбит $\mathscr{C}$.

В $\S 1.5$ мы связали с каждым симплициальньм комплексом $K^{n-1}$ на $m$ вершинах два канонических кубических подкомплекса в $I^{m}$, а именно, $\operatorname{cub}(K)(21)$ и сс $(K)(22)$. Обозначим соответствующие момент-угол комплексы через $\mathscr{W}_{K}$ и $\mathscr{Z}_{K}$. Таким образом, мы имеем

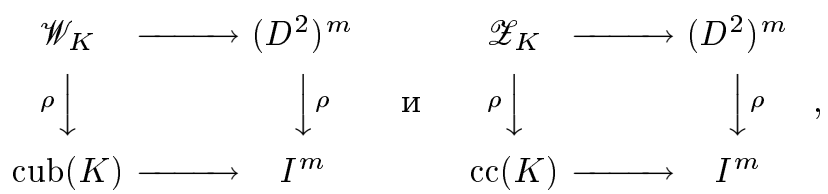

где горизонтальные стрелки являются вложениями, а вертикальные - проекциями на пространства орбит для $T^{m}$-действий. Заметим, что $\operatorname{dim} \mathscr{L}_{K}=m+n$ и $\operatorname{dim} \mathscr{W}_{K}=$ $m+n-1$. 
ЗАмечАниЕ. Пусть $K=K_{P}$ для некоторого простого многогранника $P$. Тогда из $(37)$ вытекает, что $\mathscr{Z}_{K}$ отождествляется с $\mathscr{Z}_{P}$ (или, точнее, с $i_{e}\left(\mathscr{Z}_{P}\right)$ ).

ЛЕмма 3.2.2. Если $K$ - симплициальная $(n-1)$-сфера, то $\mathscr{Z}_{K}$ является $(m+n)$-мерныцм (замкнутылм) многообразием.

ДокАЗАТЕЛЬСТво. В этом доказательстве мы будем отождествлять полиэдры $|K|$ и $|\operatorname{cone}(K)|$ с их образами $\operatorname{cub}(K) \subset I^{m}$ и сc $(K) \subset I^{m}$ при отображении $|\operatorname{cone}(K)|$ $\rightarrow I^{m}$, см. теорему 1.5.10. Для каждой вершины $\{i\} \in K$ обозначим через $F_{i}$ объединение $(n-1)$-кубов комплекса $\operatorname{cub}(K)$, содержаших $\{i\}$. По другому, $F_{i}$ есть полиэдр $\left|\operatorname{star}_{\mathrm{bs}(K)}\{i\}\right|$. Эти $F_{1}, \ldots, F_{m}$ являются аналогами гиперграней простого многогранника. Более того, если $K=K_{P}$ для некоторого $P$, то $F_{i}$ есть образ некоторой гиперграни многогранника $P$ при отображении $i_{P}: \mathscr{C}(P) \rightarrow I^{m}$ (конструкция 1.5.5). Как и в случае простых многогранников, определим "грани" комплекса сc $(K)$ как непустые пересечения "гиперграней" $F_{1}, \ldots, F_{m}$. Тогда "вершины" (т.е. непустые пересечения $n$ "гиперграней") представляют собой барицентры $(n-1)$-симплексов полиэдра $|K|$. Для каждого такого барицентра $b$ обозначим через $U_{b}$ открытое подмножество в cc $(K)$, получаемое удалением всех "граней", не содержащих $b$. Тогда $U_{b}$ отождествляется с $\mathbb{R}_{+}^{n}$, а $\rho^{-1}\left(U_{b}\right)$ гомеоморфно $T^{m-n} \times \mathbb{R}^{2 n}$. Это определяет на $n$-мерном шаге $\operatorname{cc}(K)=|\operatorname{cone}(K)|$ структуру многообразия с углами, с атласом $\left\{U_{b}\right\}$. При этом $\mathscr{Z}_{K}=\rho^{-1}(\operatorname{cc}(K))$ является многообразием с атласом $\left\{\rho^{-1}\left(U_{b}\right)\right\}$.

ПроБЛЕмА 3.2.3. Описать класс симплициальных комплексов $K$, для которых $\mathscr{Z}_{K}$ является многообразием.

Как мы увидим ниже (теорема 4.2.1), структура гомологий $\mathscr{Z}_{K}$ показывает, что если $\mathscr{Z}_{K}$ является многообразием, то $K$ есть горенштейнов* комплекс. Таким образом, решение проблемы 3.2.3 находится где-то между “симплициальными сферами” и “горенштейновыми* комплексами".

§ 3.3. Клеточные структуры на момент-угол комплексах. Здесь мы рассматриваем два клеточных разбиения полидиска $\left(D^{2}\right)^{m}$, которые задают клеточную структуру на момент-угол комплексах. Первое клеточное разбиение имеет $5^{m}$ клеток и определяет клеточное структуру (с 5 типами клеток) на любом момент-угол комплексе та $(\mathscr{C}) \subset\left(D^{2}\right)^{m}$. Второе клеточное разбиение полидиска $\left(D^{2}\right)^{m}$ имеет лишш $3^{m}$ клеток, однако определяет клеточную структуру (с 3 типами клеток) только для момент-угол комплексов $\mathscr{Z}_{K}$.

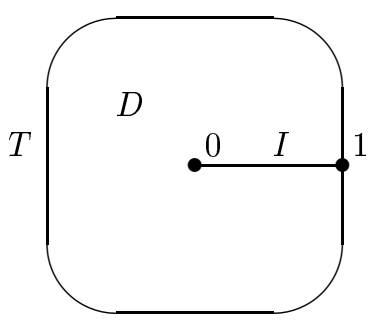

(a)

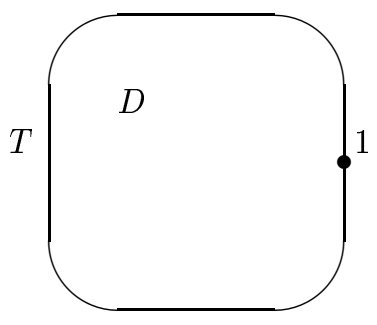

(б)

Рис. 7. Клеточные разбиения диска $D^{2}$ 
Рассмотрим клеточное разбиение диска $D^{2}$, имеюшее одну 2-клетку $D$, две 1-клетки $I, T$ и две 0 -клетки 0,1 , см. рис. 7 (а). Оно определяет клеточное разбиение полидиска $\left(D^{2}\right)^{m}$, имеющее $5^{m}$ клеток. Каждая клетка этого клеточного комплекса представляет собой произведение клеток 5 различных типов: $D_{i}, I_{i}, 0_{i}, T_{i}$ и $1_{i}, i=1, \ldots, m$. Мы будем записьвать клетки полидиска $\left(D^{2}\right)^{m}$ "словами" вида $D_{I} I_{J} 0_{L} T_{P} 1_{Q}$, где $I, J, L, P, Q$-попарно непересекаюшиеся подмножества в $[m]$ такие, что $I \cup J \cup L \cup P \cup Q=[m]$. Иногда мы будем опускать последний сомножитель $1_{Q}$, так что в наших обозначениях $D_{I} I_{J} 0_{L} T_{P}=D_{I} I_{J} 0_{L} T_{P} 1_{[m] \backslash I \cup J \cup L \cup P}$. Замыкание клетки $D_{I} I_{J} 0_{L} T_{P} 1_{Q}$ гомеоморфно произведению \#I дисков, \#J отрезков и \#P окружностей. Построенное клеточное разбиение полидиска $\left(D^{2}\right)^{m}$ позволяет отождествлять момент-угол комплексы как некоторые клеточные подкомплексы в $\left(D^{2}\right)^{m}$.

Лемма 3.3.1. Для любого кубического подкомплекса $\mathscr{C}$ в $I^{m}$ соответству-

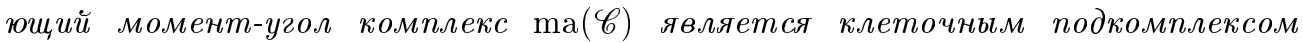
в $\left(D^{2}\right)^{m}$.

ДокАЗАТЕльство. Действительно, та( $\mathscr{C})$ представляет собой объединение некоторых "момент-угол" блоков $B_{I \subset J}(38)$, а каждый $B_{I \subset J}$ есть замыкание клетки $D_{J \backslash I} I_{\varnothing} 0_{I} T_{[m] \backslash J} 1_{\varnothing}$.

Теперь мы сосредоточимся на момент-угол комплексе $\mathscr{Z}_{K}$, соответствуюшем кубическому комплексу сc $(K) \subset I^{m}$ (см. (39)). По определению $\mathscr{Z}_{K}$ есть объединение момент-угол блоков $B_{I \subset J} \subset\left(D^{2}\right)^{m}$ с $J \in K$. Обозначим

$$
B_{J}:=B_{\varnothing \subset J}=\left\{\left(z_{1}, \ldots, z_{m}\right) \in\left(D^{2}\right)^{m}:\left|z_{j}\right|=1 \text { при } j \notin J\right\} .
$$

Тогда $B_{J}=\rho^{-1}\left(C_{J}\right)$ (напомним, что $C_{J}:=C_{\varnothing \subset J}$ ) и $B_{I \subset J} \subset B_{J}$ для любого $I \subset J$. Отсюда следует, что

$$
\mathscr{Z}_{K}=\bigcup_{J \in K} B_{J}
$$

(сравните это с замечанием после (22)).

ЗАмечАниЕ. Если $K=K_{P}$ для некоторого простого многогранника $P$ и $\# J=n$, то $B_{J}$ есть в точности $i_{e}\left(B_{v}\right)$ для $v=\bigcap_{j \in J} F_{j}$. Таким образом, $(41)$ в этом случае сводится к (37).

Заметим, что $B_{J} \cap B_{J^{\prime}}=B_{J \cap J^{\prime}}$. Это позволяет упростить клеточное разбиение из леммы 3.3 .1 в случае та $(\mathscr{C})=\mathscr{Z}_{K}$. Для этого мы заменим объединение клеток $0, I, D$ (см. рис. 7 (а)) на одну 2-мерную клетку (которую мы также будем обозначать $D$ ). Получаемое в результате клеточное разбиение диска $D^{2}$ с 3 клетками показано на рис. 7 (б). Оно определяет клеточное разбиение полидиска $\left(D^{2}\right)^{m}$ с $3^{m}$ клетками, каждая из которых представляет собой произведение клеток 3 различных типов: $D_{i}, T_{i}$ и $1_{i}, i=1, \ldots, m$. Мы будем записывать эти клетки полидиска $\left(D^{2}\right)^{m}$ словами $D_{I} T_{P} 1_{Q}$, где $I, P, Q$ - попарно различные подмножества в $[m]$ такие, что $I \cup P \cup Q=[m]$. Обозначим $D_{I} T_{P}:=D_{I} T_{P} 1_{[m] \backslash I \cup P}$. Замыкание клетки $D_{I} T_{P}$ есть произведение \#I дисков и \# $P$ окружностей. 
Лемма 3.3.2. Момент-угол комплекс $\mathscr{Z}_{K}$ является клеточнылм подкомплексом в $\left(D^{2}\right)^{m}$ относительно клеточного разбиения с $3^{m}$ клетками (рис. 7 (б)). Каждая клетка из $\mathscr{Z}_{K}$ имеет вид $D_{I} T_{P}, I \in K$.

ДокаЗАтЕльство. Так как $B_{J}=B_{\varnothing \subset J}-$ замыкание клетки $D_{J} T_{[m] \backslash J} 1_{\varnothing}$, утверждение вытекает из (41).

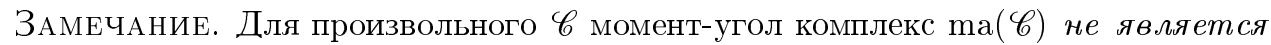
клеточньм подкомплексом в $3^{m}$-клеточном разбиении полидиска $\left(D^{2}\right)^{m}$.

По определению (конструкция 1.5.9) кубический комплекс $c с(K)$ всегда содержит вершину $(1, \ldots, 1) \in I^{m}$. Таким образом, тор $T^{m}=\rho^{-1}(1, \ldots, 1)$ содержится в $\mathscr{Z}_{K}$.

ЛЕмма 3.3.3. Вложсение $T^{m}=\rho^{-1}(1, \ldots, 1) \hookrightarrow \mathscr{Z}_{K}$ является клеточным отображением, гомотопныц отображению в точку, т.е. тор $T^{m}=\rho^{-1}(1, \ldots, 1)$ является стягиваемым клеточным подкомплексом в $\mathscr{Z}_{K}$.

ДокАЗАТЕльство. Для доказательства того факта, что $T^{m}=\rho^{-1}(1, \ldots, 1)$ является клеточным подкомплексом в $\mathscr{Z}_{K}$, мы заметим, что этот тор является замыканием $m$-клетки $D_{\varnothing} T_{[m]} \subset \mathscr{Z}_{K}$. Остается доказать, что $T^{m}$ стягиваем в $\mathscr{Z}_{K}$. Для этого мы покажем, что вложение $T^{m} \subset\left(D^{2}\right)^{m}$ гомотопно отображению в точку $(1, \ldots, 1) \in$ $T^{m} \subset\left(D^{2}\right)^{m}$. На первом шаге мы заметим, что $\mathscr{Z}_{K}$ содержит клетку $D_{1} T_{2}, \ldots, m$, замыкание которой содержит $T^{m}$ и гомеоморфно $D^{2} \times T^{m-1}$. Таким образом, наш тор $T^{m}$ может быть стянут на $1 \times T^{m-1}$ внутри $\mathscr{Z}_{K}$. На втором шаге мы замечаем, что $\mathscr{Z}_{K}$ содержит клетку $D_{2} T_{3}, \ldots, m$, замыкание которой содержит $1 \times T^{m-1}$ и гомеоморфно $D^{2} \times T^{m-2}$. Таким образом, $1 \times T^{m-1}$ может быть стянуто на $1 \times 1 \times T^{m-2}$ внутри $\mathscr{Z}_{K}$, и т. д. На $k$-м шаге мы замечаем, что $\mathscr{Z}_{K}$ содержит клетку $D_{k} T_{k+1, \ldots, m}$, замыкание которой содержит $1 \times \cdots \times 1 \times T^{m-k+1}$ и гомеоморфно $D^{2} \times T^{m-k}$. Таким образом, $1 \times \cdots \times 1 \times T^{m-k+1}$ может быть стянуто на $1 \times \cdots \times 1 \times T^{m-k}$ внутри $\mathscr{Z}_{K}$. В конце мы получим точку $1 \times \cdots \times 1$, к которой стягивается весь тор $T^{m}$.

СЛЕДСТВИЕ 3.3.4. Для любого симпличиального комплекса $K$ момент-угол комплекс $\mathscr{Z}_{K}$ односвязен.

ДокАЗАТЕльство. Действительно, 1-остов нашего клеточного разбиения $\mathscr{Z}_{K}$ содержится в торе $T^{m}=\rho^{-1}(1, \ldots, 1)$.

§.4. Конструкция Бореля и пространство Стенли-Райснера. Пусть $E T^{m}$ - стягиваемое пространство универсального главного $T^{m}$-расслоения над классифицируюшим пространством $B T^{m}$. Известно, что в качестве $B T^{m}$ можно взять произведение $m$ копий бесконечномерного проективного пространства $\mathbb{C} P^{\infty}$. Клеточное разбиение пространства $\mathbb{C} P^{\infty}$ с одной клеткой в каждой четной размерности определяет каноническое клеточное разбиение для $B T^{m}$. Когомологии $B T^{m}$ (с коэффициентами в $\mathbf{k})$ представляют собой кольцо многочленов $\mathbf{k}\left[v_{1}, \ldots, v_{m}\right], \operatorname{deg} v_{i}=2$.

ОПРЕДЕЛЕНИЕ 3.4.1. Пусть $X$-пространство с действием тора $T^{m}$. Конструкиия Бореля (в другой терминологии гомотопическое пространство орбит или ассоциированное расслоение) для $X$ есть фактор-пространство

$$
E T^{m} \times_{T^{m}} X:=E T^{m} \times X / \sim,
$$

где $(e, x) \sim\left(e g, g^{-1} x\right)$ для любых $e \in E T^{m}, x \in X, g \in T^{m}$. 
Проекция $(e, x) \rightarrow e$ определяет $E T^{m} \times_{T^{m}} X$ как тотальное пространство расслоения $E T^{m} \times_{T^{m}} X \rightarrow B T^{m}$ со слоем $X$ и структурной группой $T^{m}$. В то же время, имеется главное $T^{m}$-расслоение $E T^{m} \times X \rightarrow E T^{m} \times_{T^{m}} X$.

Далее мы будем обозначать конструкцию Бореля $E T^{m} \times_{T^{m}} X$, соответствующую $T^{m}$-пространству $X$, через $B_{T} X$. В частности, для любого симплициального комплекса $K$ на $m$ вершинах определены конструкция Бореля $B_{T} \mathscr{Z}_{K}$ и расслоение $p: B_{T} \mathscr{Z}_{K} \rightarrow B T^{m}$ со слоем $\mathscr{Z}_{K}$.

Для каждого $i=1, \ldots, m$ обозначим $i$-е координатное подпространство в $B T^{m}=$ $\left(\mathbb{C} P^{\infty}\right)^{m}$ через $B T_{i}$. Для любого подмножества $I \subset[m]$ обозначим через $B T_{I}$ произведение координатных подпространств $B T_{i}$ с $i \in I$. Очевидно, $B T_{I}$ является клеточнгм подкомплексом в $B T^{m}$, и если \# $I=k$, то $B T_{I} \cong B T^{k}$.

ОПРЕДЕЛЕНИЕ 3.4.2. Для любого симплициального комплекса $K$ мы называем клеточньй подкомплекс

$$
\bigcup_{I \in K} B T_{I} \subset B T^{m}
$$

пространством Стенли-Райснера и обозначаем его $S R(K)$.

Название объясняется следуюшим утверждением, которое непосредственно вытекает из определения 1.3.1 кольца Стенли-Райснера $\mathbf{k}(K)$.

ПРЕДЛОЖЕНИЕ 3.4.3. Алгебра клеточных кочепей $C^{*}(S R(K))$ и алгебра когомологий $H^{*}(S R(K))$ изоморфны кольиу граней $\mathbf{k}(K)$. Клеточное вложение $i: S R(K) \hookrightarrow B T^{m}$ индуцирует эпиморфизм $i^{*}: \mathbf{k}\left[v_{1}, \ldots, v_{m}\right] \rightarrow \mathbf{k}(K)=$ $\mathbf{k}\left[v_{1}, \ldots, v_{m}\right] / \mathscr{I}_{K}$ в когомологиях.

Tеорема 3.4.4. Расслоение $p: B_{T} \mathscr{Z}_{K} \rightarrow$ BT ${ }^{m}$ гомотопически әквивалентно клеточному вложению $i: S R(K) \hookrightarrow B T^{m}$. Точнее, имеется деформационная ретракиия $B_{T} \mathscr{Z}_{K} \rightarrow S R(K)$ такая, что диаграмма

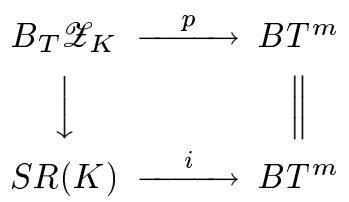

коммутативна.

ДокаЗАТЕльство. Рассмотрим разложение (41). Так как каждое подмножество $B_{J} \subset \mathscr{Z}_{K}$ является $T^{m}$-инвариантным, конструкция Бореля $B_{T} \mathscr{Z}_{K}=E T^{m} \times_{T^{m}} \mathscr{Z}_{K}$ склеивается из конструкций Бореля $E T^{m} \times_{T^{m}} B_{J}, J \in K$. Пусть \# $J=j$; тогда $B_{J} \cong\left(D^{2}\right)^{j} \times T^{m-j}$ (см. (40)). По определению конструкции Бореля $E T^{m} \times_{T^{m}} B_{J} \cong$ $\left(E T^{j} \times_{T^{j}}\left(D^{2}\right)^{j}\right) \times E T^{m-j}$. Пространство $E T^{j} \times_{T^{j}}\left(D^{2}\right)^{j}$ является пространством $\left(D^{2}\right)^{j}$-расслоения над $B T^{j}$. Отсюда следует, что имеется деформационная ретракция $E T^{m} \times_{T^{m}} B_{J} \rightarrow B T_{J}$, которая задает гомотопическую эквивалентность между ограничением проекции $p: B_{T} \mathscr{Z}_{K} \rightarrow B T^{m}$ на $E T^{m} \times_{T^{m}} B_{J}$ и клеточньм вложением $B T_{J} \hookrightarrow B T^{m}$. Эти гомотопические эквивалентности, соответствующие различным симплексам $J \in K$, согласованы между собой и определяют требуемую гомотопическую эквивалентность между $p: B_{T} \mathscr{Z}_{K} \rightarrow B T^{m}$ и $i: S R(K) \hookrightarrow B T^{m}$. 
СЛЕДСТВИЕ 3.4.5. Момент-угол комплекс $\mathscr{Z}_{K}$ является гомотопическим слоем клеточного вложения $i: S R(K) \hookrightarrow B T^{m}$.

СлЕДСТВИЕ 3.4.6. Алгебра когомологий $H^{*}\left(B_{T} \mathscr{Z}_{K}\right)$ изоморфна кольцу граней $\mathbf{k}(K)$. Проекиия $p: B_{T} \mathscr{Z}_{K} \rightarrow B T^{m}$ индуцирует эпиморфизм $p^{*}: \mathbf{k}\left[v_{1}, \ldots, v_{m}\right]$ $\rightarrow \mathbf{k}(K)=\mathbf{k}\left[v_{1}, \ldots, v_{m}\right] / \mathscr{I}_{K}$ в когомологиях.

ЗАмечАниЕ. Предыдушее утверждение в случае многогранников $\left(\mathscr{Z}_{K}=\mathscr{Z}_{P}\right)$ было доказано в [33; теорема 4.8] другими методами.

Из предыдущих конструкций извлекается следуюшая информация о гомотопических группах комплекса $\mathscr{Z}_{K}$.

Tеорема 3.4.7. (а) Комплекс $\mathscr{Z}_{K}$ является 2-связным $\left(\right.$ m.е. $\pi_{1}\left(\mathscr{Z}_{K}\right)=\pi_{2}\left(\mathscr{Z}_{K}\right)$ $=0), u \pi_{i}\left(\mathscr{Z}_{K}\right)=\pi_{i}\left(B_{T} \mathscr{Z}_{K}\right)=\pi_{i}(S R(K))$ nрu $i \geqslant 3$.

(б) Если $K=K_{P}$ и $P$ является $q$-смежсностным то то $\pi_{i}\left(\mathscr{Z}_{K}\right)=0$ при $i<2 q+1$, a $\pi_{2 q+1}\left(\mathscr{Z}_{P}\right)$ представляет собой свободную абелеву группу, образующие которой соответствуют $(q+1)$-әлементным недостающим граням комплекса $K_{P}$ (или, әквивалентно, образующим степени $(2 q+2)$ идеала $\mathscr{I}_{P}$, см. определение 1.1.20).

ДокАЗАТЕЛЬство. Заметим, что $B T^{m}=K\left(\mathbb{Z}^{m}, 2\right)$ и 3 -остов пространства $S R(K)$ совпадает с 3 -остовом $B T^{m}$. Если $P$ является $q$-смежностным, то, как следует из определения $3.4 .2,(2 q+1)$-остов пространства $S R\left(K_{P}\right)$ совпадает с $(2 q+1)$-остовом $B T^{m}$. Теперь оба утверждения вытекают из точной гомотопической последовательности отображения $i: S R(K) \rightarrow B T^{m}$ с гомотопическим слоем $\mathscr{Z}_{K}$ (следствие 3.4 .5$)$.

ЗАмечАниЕ. Скажем, что симплициальньй комплекс $K$ на множестве $[m]$ является $q$-смежсностным, если любое $q$-Элементное подмножество в $[m]$ является его симплексом (это определение является очевидным обобшением понятия $q$-смежностного симплициального многогранника на произвольные симплициальные комплексы). Тогда вторая часть теоремы 3.4.7 имеет место для произвольного $q$-смежностного симплициального комплекса.

Предположим теперь, что $K=K_{P}$ для некоторого простого $n$-многогранника $P$, и пусть $M^{2 n}$ - квазиторическое многообразие над $P$ с характеристическим отображением $\ell$. Тогда мы имеем свободно действуюшую на $\mathscr{Z}_{P}$ подгруппу $H(\ell) \subset T^{m}$ и главное $T^{m-n}$-расслоение $\mathscr{Z}_{P} \rightarrow M^{2 n}$ (предложение 3.1.5).

ПРЕДЛОЖЕНИЕ 3.4.8. Конструкиия Бореля $E T^{n} \times_{T^{n}} M^{2 n}$ гомотопически эквивалентна $B_{T} \mathscr{Z}_{P}$.

ДокАЗАТЕЛЬСтво. Так как $H(\ell)$ свободно действует на $\mathscr{Z}_{P}$, мы имеем

$$
\begin{aligned}
B_{T^{2}} \mathscr{Z}_{P} & =E T^{m} \times_{T^{m}} \mathscr{Z}_{P} \\
& =E H(\ell) \times\left(E\left(T^{m} / H(\ell)\right) \times_{T^{m} / H(\ell)} \mathscr{Z}_{P} / H(\ell)\right) \simeq E T^{n} \times_{T^{n}} M^{2 n} .
\end{aligned}
$$

ТЕОрема 3.4 .9 [33; теорема 4.12]. Спектральная последовательность ЛереСерра расслоения

$$
E T^{n} \times_{T^{n}} M^{2 n} \rightarrow B T^{n}
$$

со слоем $M^{2 n}$ вырожсдается в члене $E_{2}$, m.е. $E_{2}^{p, q}=E_{\infty}^{p, q}$. 
ДокАЗАТЕЛЬСтвО. Дифференциалы спектральной последовательности тривиальны по соображениям размерности, так как и $B T^{n}$ и $M^{2 n}$ имеют клетки лишь в четных размерностях (см. предложение 2.2.5).

СлЕДСТВИЕ 3.4.10. Проекция (42) индуцирует мономорфизм $\mathbf{k}\left[t_{1}, \ldots, t_{n}\right] \rightarrow$ $\mathbf{k}(K)$ в когомологиях. Вложение слоя $M^{2 n} \hookrightarrow E T^{n} \times_{T^{n}} M^{2 n}$ индуцирует әпиморфизм $\mathbf{k}(K) \rightarrow H^{*}\left(M^{2 n}\right)$.

Теперь мы можем доказать утверждения из $§ 2.2$.

ДОКАЗАТЕЛЬСТВО ЛЕММЫ 2.2.6 и ТЕОРЕМЫ 2.2.7. Мономорфизм $H^{*}\left(B T^{n}\right)=$ $\mathbf{k}\left[t_{1}, \ldots, t_{n}\right] \rightarrow \mathbf{k}(K)=H^{*}\left(E T^{n} \times_{T^{n}} M^{2 n}\right)$ переводит $t_{i}$ в $\theta_{i}, i=1, \ldots, n$. Так как $\mathbf{k}(K)$ является свободным $\mathbf{k}\left[t_{1}, \ldots, t_{n}\right]$-модулем (заметим, что это вытекает из теоремы 3.4.9, так что нам не нужно использовать теорему 1.3 .8$), \theta_{1}, \ldots, \theta_{n}$ есть регулярная последовательность. Отсюда вытекает, что ядро эпиморфизма $\mathbf{k}(K) \rightarrow H^{*}\left(M^{2 n}\right)$ есть в точности $\mathscr{J}_{\ell}=\left(\theta_{1}, \ldots, \theta_{n}\right)$.

$\S$ 3.5. Обобщения, аналоги и дополнительные комментарии. Ряд важных конструкций нашего обзора (кубический комплекс сс $(K)$, момент-угол комплекс $\mathscr{Z}_{K}$, конструкция Бореля $B_{T} \mathscr{Z}_{K}$, пространство Стенли-Райснера $S R(K)$, а также дополнение $U(K)$ конфигурации координатных подпространств из $\S 5.2)$ допускают единообразное изложение при помоши следуюшей конструкции, предложенной Н. Стрикландом.

Конструкция 3.5.1. Пусть $X$ - некоторое топологическое пространство, $W$ - его подпространство и $K$ - симплициальный комплекс на множестве вершин $[m]$. Введем следуюшее подмножество в произведении $m$ экземпляров $X$ :

$$
K_{\bullet}(X, W)=\bigcup_{I \in K}\left(\prod_{i \in I} X \times \prod_{i \notin I} W\right) .
$$

ПримеР 3.5.2. 1. $\operatorname{cc}(K)=K_{\bullet}\left(I^{1}, 1\right)($ см. $(22))$.

2. $\mathscr{Z}_{K}=K_{\bullet}\left(D^{2}, S^{1}\right)($ cм. $(41))$.

3. $S R(K)=K_{\bullet}\left(\mathbb{C} P^{\infty}, *\right)$ (см. определение 3.4.2).

4. $B_{T} \mathscr{Z}_{K}=K_{\bullet}\left(E S^{1} \times_{S^{1}} D^{2}, E S^{1} \times_{S^{1}} D^{2}\right)$ (см. доказательство теоремы 3.4.4).

Отметим, что конструкция 3.5.1 естественно обобщается на случай набора из $m$ пар $\left(X_{1}, W_{1}\right), \ldots,\left(X_{m}, W_{m}\right)$; при этом можно также использовать расслоенное произведение вместо декартова.

При помощи конструкции 3.5.1 Н. Стрикланд независимо получил часть теоремы 1.5.10, касаюшуюся комплекса сc $(K)$, и теорему 5.2.5.

В нашей работе большинство конструкций так или иначе связано с действием тора, представляющего собой произведение окружностей $S^{1}$. Эти конструкции допускают естественные $\mathbb{Z} / 2$-аналоги. Для этого мы заменим тор $T^{m}$ на его "вешественный аналог", т.е. группу $(\mathbb{Z} / 2)^{m}$. Тогда стандартный куб $I^{m}=[0,1]^{m}$ представляет собой пространство орбит для действия $(\mathbb{Z} / 2)^{m}$ на большем кубе $[-1,1]^{m}$, который является "вешественным аналогом" полидиска $\left(D^{2}\right)^{m} \subset \mathbb{C}^{m}$. Теперь по кубическому подкомплексу $\mathscr{C} \subset I^{m}$ мы можем построить $(\mathbb{Z} / 2)^{m}$-симметричный кубический комплекс в $[-1,1]^{m}$ аналогично тому, как это делалось в определении 3.2.1. В частности, для 
любого симплищиального комплекса $K$ на множестве вершин $[m]$ можно определить кубические комплексы $\mathbb{R} \mathscr{Z}_{K}$ и $\mathbb{R} \mathscr{W}_{K}$, которые являются "вещественньми аналогами" момент-угол комплексов $\mathscr{Z}_{K}$ и $\mathscr{W}_{K}(39)$. В обозначениях конструкции 3.5.1 мы имеем

$$
\mathbb{R}_{\mathscr{Z}_{K}}=K_{\bullet}([-1,1],\{-1,1\}) .
$$

Если $K$ является симплициальной $(n-1)$-сферой, то $\mathbb{R} \mathscr{Z}_{K}$ является $n$-мерным многообразием (доказательство аналогично доказательству леммы 3.2.2). Таким образом, по каждой симплициальной сфере $K^{n-1}$ с $m$ вершинами мы можем построить $(\mathbb{Z} / 2)^{m}$-симметричное $n$-многообразие, снабженное $(\mathbb{Z} / 2)^{m}$-инвариантньм кубическим разбиением. Этот класс кубических многообразий может быть полезен при построении кубического аналога комбинаторной теории $f$-векторов симплипиальных комплексов (см. также [83]). Наконец, вещественньй аналог $\mathbb{R} \mathscr{Z}_{P}$ многообразия $\mathscr{Z}_{P}$ (соответствующего случаю многогранной симплищиальной сферы $K^{n-1}$ ) известен как универсальное абелево накрытие многогранника $P^{n}$, рассматриваемого как орбифолд (или многообразие с углами), см. $[43 ; \S 4.5]$. В работе [47] многообразия $\mathbb{R} \mathscr{Z}_{P}$ и $\mathscr{Z}_{P}$ интерпретируются как конфигурационные пространства для плоских и пространственных иарнирных механизмов.

Замена группы $T^{n}$ на $(\mathbb{Z} / 2)^{n}$ в определении 2.2.1 приводит к вешественным аналогам квазиторических многообразий, которые были введены в [33] под названием малье накрытия ${ }^{4}$. Таким образом, каждое малое накрытие простого многогранника $P^{n}$ представляет собой некоторое многообразие $M^{n}$ с действием $(\mathbb{Z} / 2)^{n}$ и пространством орбит $P^{n}$. Название объясняется тем, что любое разветвленноенакрытие многогранника $P^{n}$ гладким многообразием имеет по крайней мере $2^{n}$ листов. Малые накрытия изучались в [33] параллельно с квазиторическими многообразиями, и большинство результатов из [33] для квазиторических многообразий, приведенных в $\S 2.2$, имеют аналоги для малых накрытий. С другой стороны, каждое малое накрытие является фактор-пространством универсального накрытия $\mathbb{R} \mathscr{Z}_{P}$ по некоторому свободному действию групшы $(\mathbb{Z} / 2)^{m-n}$.

Важный класс малых накрытий над трехмерными простыми многогранниками $P^{3}$ был рассмотрен в работе [48]. Можно показать, что простой многогранник $P^{3}$ допускает раскраску двумерных граней в три цвета так, что любые две смежные грани имеют различньй цвет тогда и только тогда, когда каждая его двумерная грань содержит четное число ребер. Каждая такая раскраска $\varrho$ определяет некоторое квазиторическое многообразие $M^{6}(\varrho)$ и малое накрытие $M^{3}(\varrho)$. В [48] показано, что каждое многообразие $M^{3}(\varrho)$ допускает эквивариантное вложение в $\mathbb{R}^{4}=\mathbb{R}^{3} \times \mathbb{R}$ со стандартным действием $(\mathbb{Z} / 2)^{3}$ на $\mathbb{R}^{3}$ и тривиальным действием на $\mathbb{R}$. Там же показано, что такие многообразия $M^{3}(\varrho)$ получаются из набора трехмерных торов при помоши операций эквивариантной связной суммы и әквивариантной перестройки Дена.

Можно построить также кватернионньй аналог момент-угол комплексов, заменяя $T^{n}$ на кватернионный тор $S p(1)^{n} \cong\left(S^{3}\right)^{n}$. Развитие кватернионного аналога торических и квазиторических многообразий, бесспорно, представляет большой интерес. На это указывают, например, важные результаты из [16].

Приведем теперь приложение описанных выше конструкций к случаю общей группा $G$.

\footnotetext{
${ }^{4} \mathrm{~B}$ оригинале small covers.
} 
ПРИМЕР 3.5.3 (классифишируюшее пространство групшы $G$ ). Пусть $K$ - некоторьй симплициальный комплекс на множестве $[m]$. Положим $\mathscr{Z}_{K}(G):=K_{\bullet}(\operatorname{cone}(G), G)$ (см. конструкцию 3.5.1), где cone $(G)$ - конус над $G$. По построению на $\mathscr{Z}_{K}(G)$ действует группа $G^{m}$ с пространством орбит cone $K$. Диагональное вложение $G \hookrightarrow G^{m}$ задает свободное действие групшы $G$ на $\mathscr{Z}_{K}(G)$.

Пусть теперь $K_{0} \subset K_{1} \subset \cdots \subset K_{i} \subset \cdots$ - последовательность вложенных симплициальных комплексов таких, что $K_{i}$ является $q_{i}$-смежностным, где $q_{i} \rightarrow \infty$ при $i \rightarrow \infty$. Такую последовательность можно получить, например, полагая $K_{i+1}:=$ $K_{i} * K$ (см. конструкцию 1.2 .5$)$, где $K_{0}$ и $K$ - любые два симплициальных комплекса. На пространстве $\lim \mathscr{Z}_{K_{i}}(G)$ группа $G$ действует свободно, и соответствующее фактор-пространство дает реализацию классифицирующего пространства $B G$. Таким образом получается фильтрация в универсальном расслоении $E G \rightarrow B G$ :

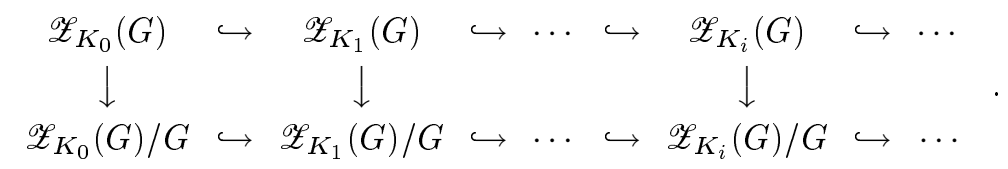

Известная фильтрации Милнора в универсальном расслоении групшы $G$ соответствует случаю $K_{i}=\Delta^{i}$.

\section{Глава 4. Когомологии момент-угол комплексов и комбинаторика симплициальных многообразий}

§4.1. Спектральная последовательность Эйленберга-Мура. В работе 1966 г. [39] Эйленбергом и Муром была построена спектральная последовательность, которая приобрела большое значение в алгебраической топологии. Эта спектральная последовательность может рассматриваться как результат обобщения подхода Адамса к вычислению когомологий пространств петель [1]. В 1960-70-х годах спектральная последовательность Эйленберга-Мура позволила получить важные результаты о когомологиях пространств петель и однородных пространств для групп Ли. В нашей работе мы описываем новые приложения этой спектральной последовательности к комбинаторным проблемам. Этот параграф̆ содержит необходимую информацию о спектральной последовательности; здесь мы следуем работе Смита [77].

Вообще говоря, имеется две спектральные последовательности Эйленберга-Мура: алгебраическая и топологическая.

Теорема 4.1.1 (Эйленберг-Мур [77; теорема 1.2]). Пусть А - дифференииальная градуированная k-алгебра и $M, N$ - дифференциальные градуированные А-модули. Тогда существует спектральная последовательность $\left\{E_{r}, d_{r}\right\}$, сходящаяся $\kappa \operatorname{Tor}_{A}(M, N)$, илен $E_{2}$ которой имеет вид

$$
E_{2}^{-i, j}=\operatorname{Tor}_{H[A]}^{-i, j}(H[M], H[N]), \quad i, j \geqslant 0,
$$

где $H[\cdot]$ обозначает алгебру (модуль) когомологий.

Спектральная последовательность из теоремы 4.1.1 располагается во втором квадранте, и дифференциал $d_{r}$ прибавляет $(r, 1-r)$ к бистепени. Эта спектральная последовательность называется (алгебраической) спектральной последовательностью 
Эйленберга-Мура. Она определяет убывающую фильтрацию $\left\{F^{-p} \operatorname{Tor}_{A}(M, N)\right\}$ в $\operatorname{Tor}_{A}(M, N)$, удовлетворяюшую

$$
E_{\infty}^{-p, n+p}=F^{-p}\left(\sum_{-i+j=n} \operatorname{Tor}_{A}^{-i, j}(M, N)\right) / F^{-p+1}\left(\sum_{-i+j=n} \operatorname{Tor}_{A}^{-i, j}(M, N)\right)
$$

Топологические приложения теоремы 4.1.1 возникают в случае, когда $A, M, N$ являются алгебрами сингулярных (или клеточных) коцепей некоторых топологических пространств. Классическая ситуация описывается коммутативным квадратом

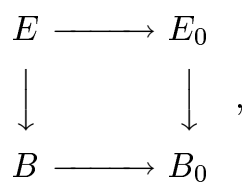

где $E_{0} \rightarrow B_{0}-$ расслоение Серра со слоем $F$ над односвязной базой $B_{0}$, а $E \rightarrow B-$ расслоение, индуцированное непрерывным отображением $B \rightarrow B_{0}$. Для любого пространства $X$ будем обозначать через $C^{*}(X)$ алгебру сингулярных коцепей для $X$ или алгебру клеточных коцепей (в случае, если $X$ является клеточным комплексом). Очевидно, $C^{*}\left(E_{0}\right)$ и $C^{*}(B)$ являются $C^{*}\left(B_{0}\right)$-модулями. При этих предположениях имеет место следующее утверждение.

ЛЕмма 4.1.2 [77; предложение 3.4]. $\operatorname{Tor}_{C^{*}\left(B_{0}\right)}\left(C^{*}\left(E_{0}\right), C^{*}(B)\right)$ естественным образом наделяется структурой алгебры, и имеет место канонический изоморфизм алгебр

$$
\operatorname{Tor}_{C^{*}\left(B_{0}\right)}\left(C^{*}\left(E_{0}\right), C^{*}(B)\right) \rightarrow H^{*}(E) .
$$

Применяя теорему 4.1.1 в случае $A=C^{*}\left(B_{0}\right), M=C^{*}\left(E_{0}\right), N=C^{*}(B)$ и принимая во внимание лемму 4.1.2, мы получаем следующее утверждение.

Теорема 4.1.3 (Эйленберг-Мур). Существует спектральная последовательность $\left\{E_{r}, d_{r}\right\}$ коммутативных алгебр такая, что

(a) $E_{r} \Rightarrow H^{*}(E)$;

(б) $E_{2}^{-i, j}=\operatorname{Tor}_{H^{*}\left(B_{0}\right)}^{-i, j}\left(H^{*}\left(E_{0}\right), H^{*}(B)\right)$.

Спектральная последовательность из теоремы 4.1.3 назьвается (топологической) спектральной последовательностью Эйленберга-Мура. В важном частном случае, когда $B$ есть точка (см. (43)), мы получаем

СлЕДСтвИЕ 4.1.4. Пусть $E \rightarrow B$ - расслоение над односвязной базой $B$ со слоем F. Тогда существует спектральная последовательность $\left\{E_{r}, d_{r}\right\}$ коммутативных алгебр такая, что

(a) $E_{r} \Rightarrow H^{*}(F)$,

(б) $E_{2}=\operatorname{Tor}_{H^{*}(B)}\left(H^{*}(E), \mathbf{k}\right)$.

Мы будем назьвать спектральную последовательность из следствия 4.1.4 cneктральной последовательностью Эйленберга-Мура расслоения $E \rightarrow B$. 
ПримеР 4.1.5. Пусть $M^{2 n}$ - квазиторическое многообразие над $P^{n}$. Рассмотрим спектральную последовательность Эйленберга-Мура расслоения $E T^{n} \times T^{n} M^{2 n} \rightarrow$ $B T^{n}$ со слоем $M^{2 n}$. В силу предложения 3.4.8 $H^{*}\left(E T^{n} \times_{T^{n}} M^{2 n}\right)=H^{*}\left(B_{T^{2}} \mathscr{Z}_{P}\right) \cong$ $\mathbf{k}\left(P^{n}\right)$. Мономорфизм

$$
\mathbf{k}\left[t_{1}, \ldots, t_{n}\right]=H^{*}\left(B T^{n}\right) \rightarrow H^{*}\left(E T^{n} \times_{T^{n}} M^{2 n}\right)=\mathbf{k}\left(P^{n}\right)
$$

переводит $t_{i}$ в $\theta_{i}, i=1, \ldots, n$, см. $(27)$. Член $E_{2}$ спектральной последовательности Эйленберга-Мура имеет вид

$$
E_{2}^{*, *}=\operatorname{Tor}_{H^{*}\left(B T^{n}\right)}^{*, *}\left(H^{*}\left(E T^{n} \times_{T^{n}} M^{2 n}\right), \mathbf{k}\right)=\operatorname{Tor}_{\mathbf{k}\left[t_{1}, \ldots, t_{n}\right]}^{*, *}\left(\mathbf{k}\left(P^{n}\right), \mathbf{k}\right) .
$$

Так как $\mathbf{k}\left(P^{n}\right)$ является свободным $\mathbf{k}\left[t_{1}, \ldots, t_{n}\right]$-модулем, мы имеем

$$
\begin{aligned}
\operatorname{Tor}_{\mathbf{k}\left[t_{1}, \ldots, t_{n}\right]}^{*, *}\left(\mathbf{k}\left(P^{n}\right), \mathbf{k}\right) & =\operatorname{Tor}_{\mathbf{k}\left[t_{1}, \ldots, t_{n}\right]}^{0, *}\left(\mathbf{k}\left(P^{n}\right), \mathbf{k}\right) \\
& =\mathbf{k}\left(P^{n}\right) \otimes_{\mathbf{k}\left[t_{1}, \ldots, t_{n}\right]} \mathbf{k}=\mathbf{k}\left(P^{n}\right) /\left(\theta_{1}, \ldots, \theta_{n}\right) .
\end{aligned}
$$

Следовательно, $E_{2}^{0, *}=\mathbf{k}\left(P^{n}\right) / \mathscr{J}_{\ell}$ и $E_{2}^{-p, *}=0$ при $p>0$. Отсюда вытекает, что спектральная последовательность Эйленберга-Мура вырождается в члене $E_{2}$ и $H^{*}\left(M^{2 n}\right)$ $=\mathbf{k}\left(P^{n}\right) / J_{\ell}$ в соответствии с теоремой 2.2.7.

$\S$ 4.2. Когомологии момент-угол комплекса $\mathscr{Z}_{K}$ : случай общего $K$. Здесь мы применяем спектральную последовательность Эйленберга-Мура для вычисления алгебры когомологий момент-угол комплекса $\mathscr{Z}_{K}$ с коэффищиентами в поле $\mathbf{k}$. Тем самым мы описываем $H^{*}\left(\mathscr{Z}_{K}\right)$ как биградуированную алгебру. Соответствующие биградуированные числа Бетти являются важными комбинаторньми инвариантами комплекса $K$.

ТеОРема 4.2.1. Имеет место следующий изоморфизм градуированных алгебр:

$$
H^{*}\left(\mathscr{Z}_{K}\right) \cong \operatorname{Tor}_{\mathbf{k}\left[v_{1}, \ldots, v_{m}\right]}(\mathbf{k}(K), \mathbf{k})
$$

В частности,

$$
H^{p}\left(\mathscr{Z}_{K}\right) \cong \sum_{-i+2 j=p} \operatorname{Tor}_{\mathbf{k}\left[v_{1}, \ldots, v_{m}\right]}^{-i, k}(\mathbf{k}(K), \mathbf{k})
$$

ДокАЗАтЕльство. Рассмотрим спектральную последовательность ЭйленбергаМура коммутативного квадрата

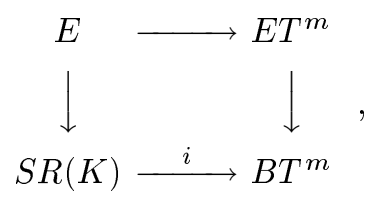

где левая вертикальная стрелка обозначает расслоение, индуцированное отображением $i$. Следствие 3.4 .5 показьвает, что $E$ гомотопически эквивалентно комплекcy $\mathscr{Z}_{K}$. 
В силу предложения 3.4 .3 отображение $i: S R(K) \hookrightarrow B T^{m}$ индуцирует эпиморфизм $i^{*}: C^{*}\left(B T^{m}\right)=\mathbf{k}\left[v_{1}, \ldots, v_{m}\right] \rightarrow \mathbf{k}(K)=C^{*}(S R(K))$, где $C^{*}(\cdot)$ обозначает алгебру клеточных коцепей. Используя каноническую коцепную эквивалентность $C^{*}\left(E T^{m}\right) \simeq \mathbf{k}$, получаем изоморфизм

$$
\operatorname{Tor}_{C^{*}\left(B T^{m}\right)}\left(C^{*}(S R(K)), C^{*}\left(E T^{m}\right)\right) \cong \operatorname{Tor}_{\mathbf{k}\left[v_{1}, \ldots, v_{m}\right]}(\mathbf{k}(K), \mathbf{k})
$$

Спектральная последовательность Эйленберга-Мура коммутативного квадрата (44) имеет

$$
E_{2}=\operatorname{Tor}_{H^{*}\left(B T^{m}\right)}\left(H^{*}(S R(K)), H^{*}\left(E T^{m}\right)\right)
$$

и сходится к $\operatorname{Tor}_{C^{*}\left(B T^{m}\right)}\left(C^{*}(S R(K)), C^{*}\left(E T^{m}\right)\right)$ (теорема 4.1.1). Так как

$$
\operatorname{Tor}_{H^{*}\left(B T^{m}\right)}\left(H^{*}(S R(K)), H^{*}\left(E T^{m}\right)\right)=\operatorname{Tor}_{\mathbf{k}\left[v_{1}, \ldots, v_{m}\right]}(\mathbf{k}(K), \mathbf{k}),
$$

изоморфизм (45) показьвает, что спектральная последовательность вырождается в члене $E_{2}$, т.е. $E_{2}=E_{\infty}$. Из леммы 4.1.2 вытекает, что модуль

$$
\operatorname{Tor}_{C^{*}\left(B T^{m}\right)}\left(C^{*}(S R(K)), C^{*}\left(E T^{m}\right)\right)
$$

является алгеброй, изоморфной алгебре $H^{*}\left(\mathscr{Z}_{K}\right)$, что завершает доказательство.

Теорема 4.2.1 задает в когомологиях комплекса $\mathscr{Z}_{K}$ структуру биградуированной алгебры и показывает, что соответствуюшие биградуированные числа Бетти $b^{-i, 2 j}\left(\mathscr{Z}_{K}\right)$ совпадают с числами Бетти кольца $\mathbf{k}(K)$, определенными в $(17)$. Используя резольвенту Кошуля для $\mathbf{k}$ и лемму 1.4.6, мы получаем

ТЕОРема 4.2.2. Имеет место следующий изоморфизм биградуированных алгебр:

$$
H^{*, *}\left(\mathscr{Z}_{K}\right) \cong H\left[\Lambda\left[u_{1}, \ldots, u_{m}\right] \otimes \mathbf{k}(K), d\right],
$$

где биградуированная структура и дифференциал в правой части определяются формулами (16).

Далее мы будем обозначать мономы

$$
u_{i_{1}} \ldots u_{i_{p}} v_{j_{1}} \ldots v_{j_{q}} \in\left[\Lambda\left[u_{1}, \ldots, u_{m}\right] \otimes \mathbf{k}(K), d\right]
$$

не содержащие квадратов, через $u_{I} v_{J}$, где $I=\left\{i_{1}, \ldots, i_{p}\right\}, J=\left\{j_{1}, \ldots, j_{q}\right\}$. Заметим, что bideg $u_{I} v_{J}=(-p, 2(p+q))$.

ЗАмечАниЕ. Так как дифференциал $d$ в (16) не меняет второй градуировки, дифференциальная биградуированная алгебра $\left[\Lambda\left[u_{1}, \ldots, u_{m}\right] \otimes \mathbf{k}(K), d\right]$ распадается в сумму дифференциальных алгебр, состояших из элементов с фиксированной второй градуировкой.

СЛЕДСТВИЕ 4.2.3. Спектральная последовательность Лере-Серра главного $T^{m}$-расслоения $E T^{m} \times \mathscr{Z}_{K} \rightarrow B_{T} \mathscr{Z}_{K}$ вырождается в члене $E_{3}$. 
ДокАЗАТЕльство. Рассматриваемая спектральная последовательность сходится к $H^{*}\left(E T^{m} \times \mathscr{Z}_{K}\right)=H^{*}\left(\mathscr{Z}_{K}\right)$ и имеет

$$
E_{2}=H^{*}\left(B_{T} \mathscr{Z}_{K}\right) \otimes H^{*}\left(T^{m}\right)=\Lambda\left[u_{1}, \ldots, u_{m}\right] \otimes \mathbf{k}(K) .
$$

Дифференциал в члене $E_{2}$ действует как в (16). Следовательно,

$$
E_{3}=H\left[E_{2}, d\right]=H\left[\Lambda\left[u_{1}, \ldots, u_{m}\right] \otimes \mathbf{k}(K)\right]=H^{*}\left(\mathscr{Z}_{K}\right)
$$

(последнее тождество вытекает из теоремы 4.2.2).

Конструкция 4.2.4. Пусть $A^{-q}(K) \subset \Lambda\left[u_{1}, \ldots, u_{m}\right] \otimes \mathbf{k}(K)$ - подпространство, порожденное мономами $u_{I}$ и $u_{I} v_{J}$, где $J$ - симплекс комплекса $K, \# I=q$ и $I \cap J=\varnothing$. Положим

$$
A^{*}(K)=\bigoplus_{q=0}^{m} A^{-q}(K) .
$$

Так как $d u_{i}=v_{i}$, мы имеем $d\left(A^{-q}(K)\right) \subset A^{-q+1}(K)$. Следовательно, $A^{*}(K)$ - коцепной подкомплекс в $\left[\Lambda\left[u_{1}, \ldots, u_{m}\right] \otimes \mathbf{k}(K), d\right]$. Более того, $A^{*}(K)$ наследует биградуированную структуру из $\Lambda\left[u_{1}, \ldots, u_{m}\right] \otimes \mathbf{k}(K)$ с дифференшиалом $d$, прибавляюшем $(1,0)$ к бистепени. Итак, мы имеем аддитивное вложение (т.е. мономорфизм биградуированных модулей) $i_{a}: A^{*}(K) \hookrightarrow \Lambda\left[u_{1}, \ldots, u_{m}\right] \otimes \mathbf{k}(K)$. Наконец, $A^{*}(K)$ очевидным образом является алгеброй, но не подалгеброй алгебры $\Lambda\left[u_{1}, \ldots, u_{m}\right] \otimes \mathbf{k}(K)$ (так как, например, $v_{1}^{2}=0$ в $A^{*}(K)$, но не в $\Lambda\left[u_{1}, \ldots, u_{m}\right] \otimes \mathbf{k}(K)$ ). Тем не менее, мы имеем мультипликативную проекиию (эпиморфизм биградуированных алгебр) $j_{m}: \Lambda\left[u_{1}, \ldots, u_{m}\right] \otimes \mathbf{k}(K) \rightarrow A^{*}(K)$. Аддитивное вложение $i_{a}$ и мультипликативная проекция $j_{m}$, очевидно, удовлетворяют $j_{m} \cdot i_{a}=\mathrm{id}$.

Лемма 4.2.5. Коцепные комплексы $\left[\Lambda\left[u_{1}, \ldots, u_{m}\right] \otimes \mathbf{k}(K), d\right] u\left[A^{*}(K), d\right]$ имеют одни и те же когомологии. Таким образом, имеет место следующий изоморфизм биградуированных $\mathbf{k - м о д у л е и ̆ : ~}$

$$
H\left[A^{*}(K), d\right] \cong \operatorname{Tor}_{\mathbf{k}\left[v_{1}, \ldots, v_{m}\right]}(\mathbf{k}(K), \mathbf{k}) .
$$

ДокАЗАТЕЛЬСтво. Рутинная проверка показывает, что оператор коцепной гомотопии $s$ для резольвенты Кошуля (см. доказательство предложения VII.2.1 в [56]) устанавливает коцепную гомотопическую эквивалентность между отображениями id и $i_{a} \cdot j_{m}$ Tor-алгебры на себя. Это означает, что

$$
d s+s d=\mathrm{id}-i_{a} \cdot j_{m}
$$

Мы лишь проиллюстрируем это тождество на нескольких примерах:

1) $s\left(u_{1} v_{2}\right)=u_{1} u_{2}, \quad d s\left(u_{1} v_{2}\right)=u_{2} v_{1}-u_{1} v_{2}, \quad s d\left(u_{1} v_{2}\right)=u_{1} v_{2}-u_{2} v_{1}$, следовательно, $(d s+s d)\left(u_{1} v_{2}\right)=0=\left(\mathrm{id}-i_{a} \cdot j_{m}\right)\left(u_{1} v_{2}\right)$;

2) $s\left(u_{1} v_{1}\right)=u_{1}^{2}=0, \quad d s\left(u_{1} v_{1}\right)=0, \quad d\left(u_{1} v_{1}\right)=v_{1}^{2}, \quad s d\left(u_{1} v_{1}\right)=u_{1} v_{1}$, следовательно, $(d s+s d)\left(u_{1} v_{1}\right)=u_{1} v_{1}=\left(\mathrm{id}-i_{a} \cdot j_{m}\right)\left(u_{1} v_{1}\right)$;

3) $s\left(v_{1}^{2}\right)=u_{1} v_{1}, \quad d s\left(v_{1}^{2}\right)=v_{1}^{2}, \quad d\left(v_{1}^{2}\right)=0$, следовательно, $(d s+s d)\left(v_{1}^{2}\right)=v_{1}^{2}=\left(\mathrm{id}-i_{a} \cdot j_{m}\right)\left(v_{1}^{2}\right)$. 
Теперь вспомним наше клеточное разбиение комплекса $\mathscr{Z}_{K}$ (лемма 3.3 .2 ). Клетками его являются $D_{I} T_{J}$, где $J \subset[m], I \in K$ и $I \cap J=\varnothing$. Пусть $C_{*}\left(\mathscr{Z}_{K}\right)$ и $C^{*}\left(\mathscr{Z}_{K}\right)$ обозначают соответствуюшие комплексы клеточных цепей и клеточных коцепей. Оба комплекса $C^{*}\left(\mathscr{Z}_{K}\right)$ и $A^{*}(K)$ имеют одни и те же когомологии $H^{*}\left(\mathscr{Z}_{K}\right)$. Комплекс $C^{*}\left(\mathscr{Z}_{K}\right)$ имеет базис, состоящий из коцепей $\left(D_{I} T_{J}\right)^{*}$. Как алгебра $C^{*}\left(\mathscr{Z}_{K}\right)$ порождена коцепями $T_{i}^{*}, D_{i}^{*}$ размерностей 1 и 2 соответственно, двойственными к клеткам $T_{i}$ и $D_{i}, i, j=1, \ldots, m$. В то же время, алгебра $A^{*}(K)$ порождена элементами $u_{i}, v_{i}$, $i, j=1, \ldots, m$.

Tеорема 4.2.6. Cоответствие $v_{I} u_{J} \mapsto\left(D_{I} T_{J}\right)^{*}$ устанавливает канонический изоморфизм дифференциальных градуированных алгебр $A^{*}(K)$ и $C^{*}\left(\mathscr{Z}_{K}\right)$.

ДокАЗАТЕльство. Непосредственно из определений $A^{*}(K)$ и $C^{*}\left(\mathscr{Z}_{K}\right)$ вытекает, что рассматриваемое отображение является изоморфизмом градуированњых алгебр. Таким образом, остается проверить, что оно коммутирует с дифференциалом. Пусть $d, d_{c}$ и $\partial_{c}$ обозначают дифференциалы в $A^{*}(K), C^{*}\left(\mathscr{Z}_{K}\right)$ и $C_{*}\left(\mathscr{Z}_{K}\right)$ соответственно. Так как $d\left(v_{i}\right)=0, d\left(u_{i}\right)=v_{i}$, нам необходимо показать, что $d_{c}\left(D_{i}^{*}\right)=0, d_{c}\left(T_{i}^{*}\right)=D_{i}^{*}$. Имеем $\partial_{c}\left(D_{i}\right)=T_{i}, \partial_{c}\left(T_{i}\right)=0$. Любая 2-клетка в $\mathscr{Z}_{K}$ есть либо $D_{j}$, либо $T_{j k}, k \neq j$. Тогда

$$
\left(d_{c} T_{i}^{*}, D_{j}\right)=\left(T_{i}^{*}, \partial_{c} D_{j}\right)=\left(T_{i}^{*}, T_{j}\right)=\delta_{i j}, \quad\left(d_{c} T_{i}^{*}, T_{j k}\right)=\left(T_{i}^{*}, \partial_{c} T_{j k}\right)=0,
$$

где $\delta_{i j}=1$, если $i=j$, и $\delta_{i j}=0$ иначе. Следовательно, $d_{c}\left(T_{i}^{*}\right)=D_{i}^{*}$. Далее, любая 3 -клетка в $\mathscr{Z}_{K}$ есть либо $D_{j} T_{k}$, либо $T_{j_{1} j_{2} j_{3}}$. Тогда

$$
\begin{gathered}
\left(d_{c} D_{i}^{*}, D_{j} T_{k}\right)=\left(D_{i}^{*}, \partial_{c}\left(D_{j} T_{k}\right)\right)=\left(D_{i}^{*}, T_{j k}\right)=0, \\
\left(d_{c} D_{i}^{*}, T_{j_{1} j_{2} j_{3}}\right)=\left(D_{i}^{*}, \partial_{c} T_{j_{1} j_{2} j_{3}}\right)=0 .
\end{gathered}
$$

Следовательно, $d_{c}\left(D_{i}^{*}\right)=0$.

Теорема 4.2.6 дает топологическую интерпретацию дифференциальной алгебры $\left[A^{*}(K), d\right]$. Далее мы не будем различать коцепные комплексы $A^{*}(K)$ и $C^{*}\left(\mathscr{Z}_{K}\right)$ и отождествим $u_{i}$ с $T_{i}^{*}, v_{i}$ с $D_{i}^{*}$.

Теперь вспомним, что алгебра $\left[A^{*}(K), d\right]$ является биградуированной. Изоморфизм из теоремы 4.2.6 задает биградуированную структуру в комплексе клеточных цепей $\left[C_{*}\left(\mathscr{Z}_{K}\right), \partial_{c}\right]$, г де

$$
\operatorname{bideg}\left(D_{i}\right)=(0,2), \quad \operatorname{bideg}\left(T_{i}\right)=(-1,2), \quad \operatorname{bideg}\left(1_{i}\right)=(0,0) .
$$

Дифференциал $\partial_{c}$ прибавляет $(-1,0)$ к бистепени, и клеточные гомологии комплекса $\mathscr{Z}_{K}$ также приобретают биградуированную структуру.

Далее мы будем предполагать, что основное поле $\mathbf{k}$ имеет нулевую характеристику (например, $\mathbf{k}=\mathbb{Q}$ - поле рациональных чисел). Введем биградуированные числа Бетти

$$
b_{-q, 2 p}\left(\mathscr{Z}_{K}\right)=\operatorname{dim} H_{-q, 2 p}\left[C_{*}\left(\mathscr{Z}_{K}\right), \partial_{c}\right], \quad q, p=0, \ldots, m .
$$

Теорема 4.2.6 и лемма 4.2.5 показьвают, что

$$
b_{-q, 2 p}\left(\mathscr{Z}_{K}\right)=\operatorname{dim} \operatorname{Tor}_{\mathbf{k}\left[v_{1}, \ldots, v_{m}\right]}^{-q, 2 p}(\mathbf{k}(K), \mathbf{k})=\beta^{-q, 2 p}(\mathbf{k}(K))
$$


(см. (17)). Другими словами, $b_{-q, 2 p}\left(\mathscr{Z}_{K}\right)$ равно размерности $(-q, 2 p)$-й биградуированной компоненты алгебры когомологий $H\left[\Lambda\left[u_{1}, \ldots, u_{m}\right] \otimes \mathbf{k}(K), d\right]$. Для обычных чисел Бетти $b_{k}\left(\mathscr{Z}_{K}\right)$ имеет место

$$
b_{k}\left(\mathscr{Z}_{K}\right)=\sum_{-q+2 p=k} b_{-q, 2 p}\left(\mathscr{Z}_{K}\right), \quad k=0, \ldots, m+n .
$$

Ниже мы описываем некоторые основные свойства биградуированных чисел Бетти (47).

Лемма 4.2.7. Пусть $K^{n-1}-(n-1)$-мерный симплициальный комплекс с $m=f_{0}$ вериинами и $f_{1}$ ребрами, и $\mathscr{Z}_{K}$ - соответствуюший момент-угол комплекс, $\operatorname{dim} \mathscr{Z}_{K}=m+n$. Тогда

(a) $b_{0,0}\left(\mathscr{Z}_{K}\right)=b_{0}\left(\mathscr{Z}_{K}\right)=1, b_{0,2 p}\left(\mathscr{Z}_{K}\right)=0$ npu $p>0$;

(б) $b_{-q, 2 p}=0$ при $p>m$ или $q>p$;

(в) $b_{1}\left(\mathscr{Z}_{K}\right)=b_{2}\left(\mathscr{Z}_{K}\right)=0$;

(г) $b_{3}\left(\mathscr{Z}_{K}\right)=b_{-1,4}\left(\mathscr{Z}_{K}\right)=\left(\begin{array}{c}f_{0} \\ 2\end{array}\right)-f_{1}$;

(д) $b_{-q, 2 p}\left(\mathscr{Z}_{K}\right)=0$ nрu $q \geqslant p>0$ или $p-q>n$;

(e) $b_{m+n}\left(\mathscr{Z}_{K}\right)=b_{-(m-n), 2 m}\left(\mathscr{Z}_{K}\right)$.

ДокАЗАТЕЛЬСТвО. Мы будем проводить вычисления с коцепньм комплексом $A^{*}(K) \subset \Lambda\left[u_{1}, \ldots, u_{m}\right] \otimes \mathbf{k}(K)$. Модуль $A^{*}(K)$ имеет базис из мономов $u_{J} v_{I} \mathrm{c}$ $I \in K$ и $I \cap J=\varnothing$. Так как bideg $v_{i}=(0,2)$, bideg $u_{j}=(-1,2)$, биградуированная компонента $A^{-q, 2 p}(K)$ порождена мономами $u_{J} v_{I} \mathrm{c} \# I=p-q$ и \#J $=q$. В частности, $A^{-q, 2 p}(K)=0$ при $p>m$ или $q>p$, откуда вытекает утверждение (б). Для доказательства (a) мы заметим, что $A^{0,0}(K)$ порождается 1 , в то время как любой моном $v_{I} \in A^{0,2 p}(K), p>0$, является кограницей, откуда $H^{0,2 p}\left(\mathscr{Z}_{K}\right)=0, p>0$.

Далее мы докажем утверждение (д). Каждьй моном $u_{J} v_{I} \in A^{-q, 2 p}(K)$ имеет $I \in K$, а любой симплекс из $K$ имеет размерность не более $(n-1)$. Отсюда следует, что $A^{-q, 2 p}(K)=0$ при $p-q>n$. В силу (б) $b_{-q, 2 p}\left(\mathscr{Z}_{K}\right)=0$ при $q>p$, поэтому остается доказать, что $b_{-q, 2 q}\left(\mathscr{Z}_{K}\right)=0$ при $q>0$. Модуль $A^{-q, 2 q}(K)$ порожден мономами $u_{J}, \# J=q$. Так как $d\left(u_{i}\right)=v_{i}$, легко видеть, что в $A^{-q, 2 q}(K)$ нет ненулевых коциклов. Поэтому $H^{-q, 2 q}\left(\mathscr{Z}_{K}\right)=0$.

Утверждение (в) вытекает из (д) и (49).

Из (д) также следует, что $H^{3}\left(\mathscr{Z}_{K}\right)=H^{-1,4}\left(\mathscr{Z}_{K}\right)$. Базис в $A^{-1,4}(K)$ состоит из мономов $u_{j} v_{i}, i \neq j$. Имеем $d\left(u_{j} v_{i}\right)=v_{i} v_{j}$ и $d\left(u_{i} u_{j}\right)=u_{j} v_{i}-u_{i} v_{j}$. Отсюда следует, что $u_{j} v_{i}$ является коциклом тогда и только тогда, когда $\{i, j\}$ не является 1-симплексом в $K$; в этом случае два коцикла $u_{j} v_{i}$ и $u_{i} v_{j}$ представляют один класс когомологий. Отсюда вытекает утверждение (г).

Оставшееся утверждение (е) следует из того факта, что моном $u_{I} v_{J} \in A^{*}(K)$ мак-

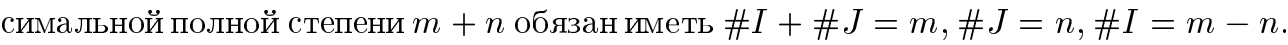

Лемма 4.2.7 показывает, что ненулевые биградуированные числа Бетти $b_{r, 2 p}\left(\mathscr{Z}_{K}\right)$, $r \neq 0$, могут появляться только в "полосе", ограниченной прямыми $p=m, r=-1$, $p+r=1$ и $p+r=n$ во втором квадранте (см. рис. 8 (а)).

Биоднородная компонента $C_{-q, 2 p}\left(\mathscr{Z}_{K}\right)$ имеет базис из клеточных цепей $D_{I} T_{J} \mathrm{c}$ $I \in K, \# I=p-q, \# J=q$. Отсюда получаем

$$
\operatorname{dim} C_{-q, 2 p}\left(\mathscr{Z}_{K}\right)=f_{p-q-1}\left(\begin{array}{c}
m-p+q \\
q
\end{array}\right)
$$




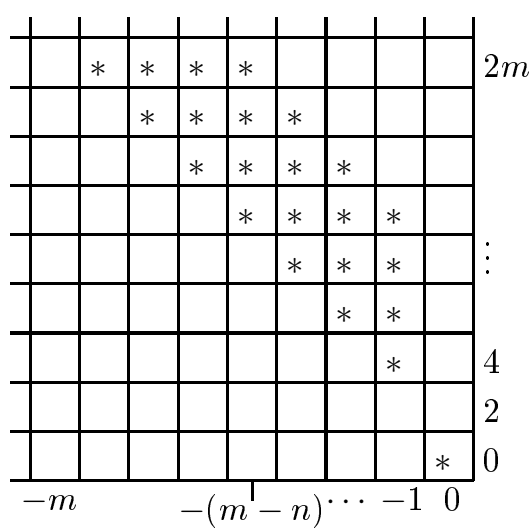

(a) произвольный $K^{n-1}$

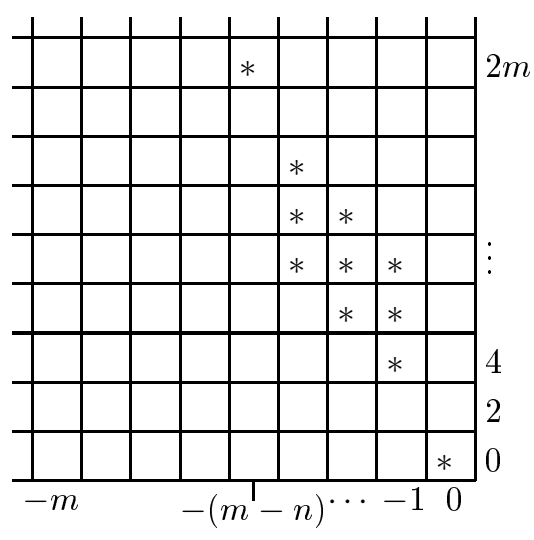

(б) $|K|=S^{n-1}$

Рис. 8. Возможные месторасположения ненулевых биградуированных чисел Бетти $b_{-q, 2 p}\left(\mathscr{Z}_{K}\right)$ (обозначены $*$ )

(мы полагаем $\left(\begin{array}{l}i \\ j\end{array}\right)=0$ при $i<j$ или $\left.j<0\right)$, где $\left(f_{0}, f_{1}, \ldots, f_{n-1}\right)-f$-вектор комплекса $K^{n-1}$ и $f_{-1}=1$. Дифференциал $\partial_{c}$ не изменяет второй градуировки, т.е.

$$
\partial_{c}: C_{-q, 2 p}\left(\mathscr{Z}_{K}\right) \rightarrow C_{-q-1,2 p}\left(\mathscr{Z}_{K}\right) .
$$

Следовательно, цепной комплекс $C_{*, *}\left(\mathscr{Z}_{K}\right)$ представляется в виде

$$
\left[C_{*, *}\left(\mathscr{Z}_{K}\right), \partial_{c}\right]=\bigoplus_{p=0}^{m}\left[C_{*, 2 p}\left(\mathscr{Z}_{K}\right), \partial_{c}\right]
$$

ЗАмечАниЕ. Аналогичное разложение имеет место и для комплекса клеточных коцепей $\left[C^{*, *}\left(\mathscr{Z}_{K}\right), d_{c}\right] \cong\left[A^{*, *}(K), d\right]$.

Рассмотрим эйлерову характеристику комплекса $\left[C_{*, 2 p}\left(\mathscr{Z}_{K}\right), \partial_{c}\right]$ :

$$
\chi_{p}\left(\mathscr{Z}_{K}\right):=\sum_{q=0}^{m}(-1)^{q} \operatorname{dim} C_{-q, 2 p}\left(\mathscr{Z}_{K}\right)=\sum_{q=0}^{m}(-1)^{q} b_{-q, 2 p}\left(\mathscr{Z}_{K}\right) .
$$

Введем порождаюший многочлен $\chi\left(\mathscr{Z}_{K} ; t\right)$ как

$$
\chi\left(\mathscr{Z}_{K} ; t\right)=\sum_{p=0}^{m} \chi_{p}\left(\mathscr{Z}_{K}\right) t^{2 p}
$$

Следующая теорема вычисляет этот многочлен в терминах $h$-вектора комплекса $K$.

ТЕорема 4.2.8. Для любого $(n-1)$-мерного симплициального комплекса $K c$ $m$ вериинами имеет место соотношение

$$
\chi\left(\mathscr{Z}_{K} ; t\right)=\left(1-t^{2}\right)^{m-n}\left(h_{0}+h_{1} t^{2}+\cdots+h_{n} t^{2 n}\right),
$$

где $\left(h_{0}, h_{1}, \ldots, h_{n}\right)$ есть -вектор комплекса $K$. 
ДокАЗАТЕЛЬСтво. Из (51) и (50) вытекает

$$
\chi_{p}\left(\mathscr{Z}_{K}\right)=\sum_{j=0}^{m}(-1)^{p-j} f_{j-1}\left(\begin{array}{c}
m-j \\
p-j
\end{array}\right) .
$$

Тогда

$$
\begin{aligned}
\chi\left(\mathscr{Z}_{K} ; t\right) & =\sum_{p=0}^{m} \chi_{p}(K) t^{2 p}=\sum_{p=0}^{m} \sum_{j=0}^{m} t^{2 j} t^{2(p-j)}(-1)^{p-j} f_{j-1}\left(\begin{array}{c}
m-j \\
p-j
\end{array}\right) \\
& =\sum_{j=0}^{m} f_{j-1} t^{2 j}\left(1-t^{2}\right)^{m-j}=\left(1-t^{2}\right)^{m} \sum_{j=0}^{n} f_{j-1}\left(t^{-2}-1\right)^{-j} .
\end{aligned}
$$

Обозначим $h(t)=h_{0}+h_{1} t+\cdots+h_{n} t^{n}$. Тогда из (7) получаем

$$
t^{n} h\left(t^{-1}\right)=(t-1)^{n} \sum_{i=0}^{n} f_{i-1}(t-1)^{-i} .
$$

Подставляя в последнем тождестве $t^{-2}$ вместо $t$ и учитьвая (54), мы окончательно получаем

$$
\frac{\chi\left(\mathscr{Z}_{K} ; t\right)}{\left(1-t^{2}\right)^{m}}=\frac{t^{-2 n} h\left(t^{2}\right)}{\left(t^{-2}-1\right)^{n}}=\frac{h\left(t^{2}\right)}{\left(1-t^{2}\right)^{n}},
$$

что эквивалентно (52).

Теорема 4.2.8 позволяет выражать числа граней симплициального комплекса в терминах биградуированных чисел Бетти соответствуюшего момент-угол комплекса $\mathscr{Z}_{K}$.

СлЕДСТвИЕ 4.2.9. Для любого симплициального комплекса $K$ әйлерова характеристика соответствующего момент-угол комплекса $\mathscr{Z}_{K}$ равна нулю.

ДокАЗАТЕЛЬСтво. Мы имеем

$$
\chi\left(\mathscr{Z}_{K}\right)=\sum_{p, q=0}^{m}(-1)^{-q+2 p} b_{-q, 2 p}\left(\mathscr{Z}_{K}\right)=\sum_{p=0}^{m} \chi_{p}\left(\mathscr{Z}_{K}\right)=\chi\left(\mathscr{Z}_{K} ; 1\right) .
$$

Теперь утверждение вытекает из (52).

ЗАмЕчАнИЕ. Другое доказательство предыдушего следствия можно получить, заметив, что диагональная подгруппа $S_{d}^{1} \subset T^{m}$ всегда действует свободно на $\mathscr{Z}_{K}$ (см. также $\S 4.4)$. Поэтому сушествует главное $S^{1}$-расслоение $\mathscr{Z}_{K} \rightarrow \mathscr{Z}_{K} / S_{d}^{1}$, откуда вытекает $\chi\left(\mathscr{Z}_{K}\right)=0$.

Тор $T^{m}=\rho^{-1}(1, \ldots, 1)$ является клеточным подкомплексом в $\mathscr{Z}_{K}$ (см. лемму 3.3.3). Клеточный коцепной комплекс $C^{*}\left(T^{m}\right) \subset C^{*}\left(\mathscr{Z}_{K}\right) \cong A^{*}(K)$ имеет базис из коцепей $\left(T_{I}\right)^{*}$ и отображается во внешнюю алгебру $\Lambda\left[u_{1}, \ldots, u_{m}\right] \subset A^{*}(K)$ при изоморфизме из теоремы 4.2.6. Отсюда вытекает, что имеет место изоморфизм модулей

$$
C^{*}\left(\mathscr{Z}_{K}, T^{m}\right) \cong A^{*}(K) / \Lambda\left[u_{1}, \ldots, u_{m}\right] .
$$


Аналогично тому, как мы делали выше, введем биградуированные относительные числа Бетти

$$
b_{-q, 2 p}\left(\mathscr{Z}_{K}, T^{m}\right)=\operatorname{dim} H^{-q, 2 p}\left[C^{*}\left(\mathscr{Z}_{K}, T^{m}\right), d\right], \quad q, p=0, \ldots, m,
$$

определим $p$-ю относительную эйлерову характеристику $\chi_{p}\left(\mathscr{Z}_{K}, T^{m}\right)$ как

$$
\chi_{p}\left(\mathscr{Z}_{K}, T^{m}\right)=\sum_{q=0}^{m}(-1)^{q} \operatorname{dim} C^{-q, 2 p}\left(\mathscr{Z}_{K}, T^{m}\right)=\sum_{q=0}^{m}(-1)^{q} b_{-q, 2 p}\left(\mathscr{Z}_{K}, T^{m}\right)
$$

и введем порождаюший многочлен $\chi\left(\mathscr{Z}_{K}, T^{m} ; t\right)$ как

$$
\chi\left(\mathscr{Z}_{K}, T^{m} ; t\right)=\sum_{p=0}^{m} \chi_{p}\left(\mathscr{Z}_{K}, T^{m}\right) t^{2 p}
$$

Теорема 4.2.10. Для любого $(n-1)$-мерного симплиииального комплекса $K$ c $m$ вершинам имеет место соотношение

$$
\chi\left(\mathscr{Z}_{K}, T^{m} ; t\right)=\left(1-t^{2}\right)^{m-n}\left(h_{0}+h_{1} t^{2}+\cdots+h_{n} t^{2 n}\right)-\left(1-t^{2}\right)^{m} .
$$

ДокАЗАТЕЛЬСтво. Так как $C^{*}\left(T^{m}\right)=\Lambda\left[u_{1}, \ldots, u_{m}\right]$ и bideg $u_{i}=(-1,2)$, мы имеем

$$
\operatorname{dim} C^{-q}\left(T^{m}\right)=\operatorname{dim} C^{-q, 2 q}\left(T^{m}\right)=\left(\begin{array}{c}
m \\
q
\end{array}\right) .
$$

Из (55), (51) и (57) получаем

$$
\chi_{p}\left(\mathscr{Z}_{K}, T^{m}\right)=\chi_{p}\left(\mathscr{Z}_{K}\right)-(-1)^{p} \operatorname{dim} C^{-p, 2 p}\left(T^{m}\right) .
$$

Следовательно,

$$
\begin{aligned}
\chi\left(\mathscr{Z}_{K}, T^{m} ; t\right) & =\chi\left(\mathscr{Z}_{K} ; t\right)-\sum_{p=0}^{m}(-1)^{p}\left(\begin{array}{c}
m \\
p
\end{array}\right) t^{2 p} \\
& =\left(1-t^{2}\right)^{m-n}\left(h_{0}+h_{1} t^{2}+\cdots+h_{n} t^{2 n}\right)-\left(1-t^{2}\right)^{m}
\end{aligned}
$$

где мы использовали (52).

Мы будем использовать теорему 4.2 .10 в $§ 4.5$.

ТЕОрема 4.2.11. Пусть $K^{n-1}$ является комплексом Коэна-Маколея, и пусть $\mathscr{J}$ - идеал в $\mathbf{k}(K)$, порожденный некоторой регулярной последовательностью длины $n$ из әлементов степени два. Тогда имеет место следующий изоморфизм алгебр:

$$
H^{*}\left(\mathscr{Z}_{K}\right) \cong \operatorname{Tor}_{\mathbf{k}\left[v_{1}, \ldots, v_{m}\right] / \mathscr{J}}(\mathbf{k}(K) / \mathscr{J}, \mathbf{k})
$$

ДокАЗАТЕльСтвО. Это вытекает из теоремы 4.2.1 и леммы 1.4.9.

Заметим, что $\mathbf{k - а л г е б р а ~} \mathbf{k}(K) / \mathscr{J}$ конечномерна. Ввиду этого обстоятельства теорема 4.2.11 иногда более удобна для вычислений (в случае комплексов Коэна-Маколея), чем обшая теорема 4.2.1. 
$\S$ 4.3. Когомологии момент-угол комплекса $\mathscr{Z}_{K}$ : случай сферического $K$. Для $K$, являюшегося симплициальной сферой, комплекс $\mathscr{Z}_{K}$ является многообразием (лемма 3.2.2). В этом параграфе мы описьваем вытекающие из этого дополнительные результаты о когомологиях $\mathscr{Z}_{K}$ и получаем интерпретацию некоторых комбинаторных проблем из главы 1 в терминах этих результатов.

Tеорема 4.3.1. Пусть $K-(n-1)$-мерная симплициальная сфера и и $\mathscr{Z}_{K}$ соответствующее момент-угол многообразие, $\operatorname{dim} \mathscr{Z}_{K}=m+n$. Тогда фундаментальный когомологический класс многообразия $\mathscr{Z}_{K}$ представляется любым мономом $\pm v_{I} u_{J} \in A^{*}(K)$ бистепени $(-(m-n), 2 m)$ таким, что I есть $(n-1)$-симплекс в $K$ и $I \cap J=\varnothing$. Знак зависит от ориентации $\mathscr{Z}_{K}$.

ДокАЗАТЕльство. Мы имеем $H^{m+n}\left(\mathscr{Z}_{K}\right)=H^{-(m-n), 2 m}\left(\mathscr{Z}_{K}\right)$ (лемма 4.2.7 (е)). По определению модуль $A^{-(m-n), 2 m}(K)$ порожден мономами $v_{I} u_{J}$ такими, что $I \in K^{n-1}, \# I=n, J=[m] \backslash I$. Любой такой моном является коциклом. Пусть $I, I^{\prime}$ - два $(n-1)$-симплекса комплекса $K^{n-1}$, имеюшие общую $(n-2)$-грань. Мы утверждаем, что соответствующие коциклы $v_{I} u_{J}, v_{I^{\prime}} u_{J^{\prime}}$, где $J=[m] \backslash I, J^{\prime}=[m] \backslash I^{\prime}$, представляют один и тот же класс когомологий (с точностью до знака). Действительно, пусть $v_{I} u_{J}=v_{i_{1}} \cdots v_{i_{n}} u_{j_{1}} \cdots u_{j_{m-n}}, v_{I^{\prime}} u_{J^{\prime}}=v_{i_{1}} \cdots v_{i_{n-1}} v_{j_{1}} u_{i_{n}} u_{j_{2}} \cdots u_{j_{m-n}}$. Так как любая $(n-2)$-грань комплекса $K$ содержится ровно в двух $(n-1)$-гранях, в $A^{*}(K) \subset \Lambda\left[u_{1}, \ldots, u_{m}\right] \otimes \mathbf{k}(K)$ имеет место тождество

$$
\begin{aligned}
d\left(v_{i_{1}} \cdots v_{i_{n-1}} u_{i_{n}} u_{j_{1}} u_{j_{2}} \cdots u_{j_{m-n}}\right) & \\
& =v_{i_{1}} \cdots v_{i_{n}} u_{j_{1}} \cdots u_{j_{m-n}}-v_{i_{1}} \cdots v_{i_{n-1}} v_{j_{1}} u_{i_{n}} u_{j_{2}} \cdots u_{j_{m-n}} .
\end{aligned}
$$

Следовательно, $\left[v_{I} u_{J}\right]=\left[v_{I^{\prime}} u_{J^{\prime}}\right]$ (как классы когомологий). Так как $K^{n-1}$ является симплициальной сферой, любые два $(n-1)$-симплекса могут быть соединены цепью симплексов такой, что любые два последовательных симплекса имеют общую $(n-2)$ грань. Отсюда следует, что все мономы $v_{I} u_{J}$ в $A^{-(m-n), 2 m}(K)$ представляют один класс когомологий (с точностью до знака). Этот класс есть образующая $H^{m+n}\left(\mathscr{Z}_{K}\right)$, т.е. фундаментальньй класс когомологий $\mathscr{Z}_{K}$.

ЗАМЕчАниЕ. В доказательстве предыдушей теоремы мы использовали два комбинаторных свойства комплекса $K^{n-1}$. Во-первых, что любая $(n-2)$-грань содержится ровно в двух $(n-1)$-гранях и, во-вторых, что любые два $(n-1)$-симплекса могут быть соединены цепю симплексов такой, что любые два последовательных симплекса имеют общую $(n-2)$-грань. Оба этих свойства имеют место для произвольного симплициального многообразия. Следовательно, если $K^{n-1}-$ симплициальное многообразие, то $b_{m+n}\left(\mathscr{Z}_{K}\right)=b_{-(m-n), 2 m}\left(\mathscr{Z}_{K}\right)=1$, и образуюшая в $H^{m+n}\left(\mathscr{Z}_{K}\right)$ может быть выбрана как описано в теореме 4.3.1.

СлЕДСТВИЕ 4.3.2. Двойственность Пуанкаре для момент-угол многообразия $\mathscr{Z}_{K}$, определяемого симпличиальной сферой $K^{n-1}$, сохраняет биградуированную структуру в (ко)гомологиях, т.e.

$$
H^{-q, 2 p}\left(\mathscr{Z}_{K}\right) \cong H_{-(m-n)+q, 2(m-p)}\left(\mathscr{Z}_{K}\right) .
$$

В частности,

$$
b_{-q, 2 p}\left(\mathscr{Z}_{K}\right)=b_{-(m-n)+q, 2(m-p)}\left(\mathscr{Z}_{K}\right) .
$$


СЛЕДСТВИЕ 4.3.3. Пусть $K^{n-1}-(n-1)$-мерная симплициальная сфера и $\mathscr{Z}_{K}-$ соответствующий момент-угол комплекс, $\operatorname{dim} \mathscr{Z}_{K}=m+n$. Тогда

(а) $b_{-q, 2 p}\left(\mathscr{Z}_{K}\right)=0$ при $q \geqslant m-n$, с единственнылм исключением $b_{-(m-n), 2 m}=1$;

(б) $b_{-q, 2 p}\left(\mathscr{Z}_{K}\right)=0$ при $p-q \geqslant n$, с единственньлм исключением $b_{-(m-n), 2 m}=1$.

Отсюда следует, что если $K^{n-1}$ - симплициальная сфера, то ненулевые биградуированные числа Бетти $b_{r, 2 p}\left(\mathscr{Z}_{K}\right), r \neq 0, r \neq m-n$, могут появляться только в "полосе", ограниченной прямьми $r=-(m-n-1), r=-1, p+r=1$ и $p+r=n-1$ во втором квадранте (см. рис. 8 (б)). Сравните это с рис. 8 (а), соответствующим случаю обшего $K$.

ПримеР 4.3.4. Пусть $K=\partial \Delta^{m-1}$. Тогда $\mathbf{k}(K)=\mathbf{k}\left[v_{1}, \ldots, v_{m}\right] /\left(v_{1} \cdots v_{m}\right)$ (пример 1.3.4). Легко видеть, что все группы когомологий $H\left[\mathbf{k}(K) \otimes \Lambda\left[u_{1}, \ldots, u_{m}\right], d\right]$ (см. теорему 4.2.2) порождаются коциклами 1 и $\left[v_{1} v_{2} \cdots v_{m-1} u_{m}\right]$. Имеем

$$
\operatorname{deg}\left(v_{1} v_{2} \cdots v_{m-1} u_{m}\right)=2 m-1,
$$

и теорема 4.3.1 показывает, что $v_{1} v_{2} \cdots v_{m-1} u_{m}$ представляет фундаментальный класс когомологий многообразия $\mathscr{Z}_{K} \cong S^{2 m-1}$ (пример 3.1.7).

ПримеР 4.3.5. Пусть $K$ - граничный комплекс $m$-угольника $P^{2}$ с $m \geqslant 4$. Мы имеем $\mathbf{k}(K)=\mathbf{k}\left[v_{1}, \ldots, v_{m}\right] / \mathscr{I}_{P}$, где $\mathscr{I}_{P}$ порожден мономами $v_{i} v_{j}, i-j \neq 0,1 \bmod m$. Комплекс $\mathscr{Z}_{K}=\mathscr{Z}_{P}$ является гладким многообразием размерности $m+2$. Числа Бетти и кольца когомологий этих многообразий были вычислены в [20]. А именно,

$$
\operatorname{dim} H^{k}\left(\mathscr{Z}_{P}\right)= \begin{cases}1 & \text { при } k=0, m+2 ; \\
0 & \text { при } k=1,2, m, m+1 ; \\
(m-2)\left(\begin{array}{c}
m-2 \\
k-2
\end{array}\right)-\left(\begin{array}{c}
m-2 \\
k-1
\end{array}\right)-\left(\begin{array}{c}
m-2 \\
k-3
\end{array}\right) & \text { при } 3 \leqslant k \leqslant m-1 .\end{cases}
$$

Например, в случае $m=5$ группа $H^{3}\left(\mathscr{Z}_{P}\right)$ имеет 5 образуюших, представленных коциклами $v_{i} u_{i+2} \in \mathbf{k}(K) \otimes \Lambda\left[u_{1}, \ldots, u_{5}\right], i=1, \ldots, 5$, а группа $H^{4}\left(\mathscr{Z}_{P}\right)$ имеет 5 образуюших, представленных коциклами $v_{j} u_{j+2} u_{j+3}, j=1, \ldots, 5$. Как вытекает из теоремы 4.3.1, произведение коциклов $v_{i} u_{i+2}$ и $v_{j} u_{j+2} u_{j+3}$ представляет нетривиальный класс когомологий в $H^{7}\left(\mathscr{Z}_{P}\right)$ тогда и только тогда, когда все индексы $i, i+2, j, j+2$, $j+3$ различны. Следовательно, для каждого из 5 классов когомологий $\left[v_{i} u_{i+2}\right]$ существует единственньй (двойственный по Пуанкаре) класс когомологий $\left[v_{j} u_{j+2} u_{j+3}\right]$ такой, что произведение $\left[v_{i} u_{i+2}\right] \cdot\left[v_{j} u_{j+2} u_{j+3}\right]$ не тривиально.

Из (51) и (59) вытекает, что для любой симплициальной сферы $K$ имеет место соотношение

$$
\chi_{p}\left(\mathscr{Z}_{K}\right)=(-1)^{m-n} \chi_{m-p}\left(\mathscr{Z}_{K}\right) .
$$

Отсюда и из (52) мы получаем

$$
\begin{aligned}
\frac{h_{0}+h_{1} t^{2}+\cdots+h_{n} t^{2 n}}{\left(1-t^{2}\right)^{n}} & =(-1)^{m-n} \frac{\chi_{m}+\chi_{m-1} t^{2}+\cdots+\chi_{0} t^{2 m}}{\left(1-t^{2}\right)^{m}} \\
& =(-1)^{n} \frac{\chi_{0}+\chi_{1} t^{-2}+\cdots+\chi_{m} t^{-2 m}}{\left(1-t^{-2}\right)^{m}} \\
& =(-1)^{n} \frac{h_{0}+h_{1} t^{-2}+\cdots+h_{n} t^{-2 n}}{\left(1-t^{-2}\right)^{n}} \\
& =\frac{h_{0} t^{2 n}+h_{1} t^{2(n-1)}+\cdots+h_{n}}{\left(1-t^{2}\right)^{n}} .
\end{aligned}
$$


Следовательно, $h_{i}=h_{n-i}$. Таким образом, соотношения Дена-Соммервилля являются следствием биградуированной двойственности Пуанкаре (59).

Тождество (52) позволяет интерпретировать различные неравенства для $f$-векторов симплициальных сфер (соответственно симплициальных многообразий) в терминах топологических инвариантов - биградуированных чисел Бетти соответствующих момент-угол многообразий (соответственно комплексов) $\mathscr{Z}_{K}$.

ПримеР 4.3.6. Как вытекает из леммы 4.2.7, для любого $K$ имеют место соотношения

$$
\begin{array}{ll}
\chi_{0}\left(\mathscr{Z}_{K}\right)=1, & \chi_{1}\left(\mathscr{Z}_{K}\right)=0, \\
\chi_{2}\left(\mathscr{Z}_{K}\right)=-b_{-1,4}\left(\mathscr{Z}_{K}\right)=-b_{3}\left(\mathscr{Z}_{K}\right), & \chi_{3}\left(\mathscr{Z}_{K}\right)=b_{-2,6}\left(\mathscr{Z}_{K}\right)-b_{-1,6}\left(\mathscr{Z}_{K}\right)
\end{array}
$$

(заметим, что $b_{4}\left(\mathscr{Z}_{K}\right)=b_{-2,6}\left(\mathscr{Z}_{K}\right)$, в то время как $b_{5}\left(\mathscr{Z}_{K}\right)=b_{-1,6}\left(\mathscr{Z}_{K}\right)+b_{-3,8}\left(\mathscr{Z}_{K}\right)$ ). Далее, из тождества (52) получаем

$$
\begin{aligned}
& h_{0}=1, \\
& h_{1}=m-n, \\
& h_{2}=\left(\begin{array}{c}
m-n+1 \\
2
\end{array}\right)-b_{3}\left(\mathscr{Z}_{K}\right), \\
& h_{3}=\left(\begin{array}{c}
m-n+2 \\
3
\end{array}\right)-(m-n) b_{-1,4}\left(\mathscr{Z}_{K}\right)+b_{-2,6}\left(\mathscr{Z}_{K}\right)-b_{-1,6}\left(\mathscr{Z}_{K}\right) .
\end{aligned}
$$

Отсюда вытекает, что неравенство $h_{1} \leqslant h_{2}(n \geqslant 4)$ из Обобщенной Гипотезы о Верхней Границе (13) для симплициальных сфер эквивалентно неравенству

$$
b_{3}\left(\mathscr{Z}_{K}\right) \leqslant\left(\begin{array}{c}
m-n \\
2
\end{array}\right) .
$$

Следуюшее неравенство $h_{2} \leqslant h_{3}(n \geqslant 6)$ из (13) эквивалентно следующему неравенству для биградуированных чисел Бетти многообразия $\mathscr{Z}_{K}$ :

$$
\left(\begin{array}{c}
m-n+1 \\
3
\end{array}\right)-(m-n-1) b_{-1,4}\left(\mathscr{Z}_{K}\right)+b_{-2,6}\left(\mathscr{Z}_{K}\right)-b_{-1,6}\left(\mathscr{Z}_{K}\right) \geqslant 0 .
$$

Мы видим, что неравенства из Обобшенной Гипотезы о Верхней Гранище интерпретируются как "топологические" неравенства для (биградуированных) чисел Бетти некоторого многообразия. Для доказательства неравенств типа (60) или (61) можно использовать топологические методы (такие как эквивариантная топология и теория Морса). Такой топологический подход к проблемам типа $g$-гипотезы или Обобщенной Гипотезы о Верхней Гранище имеет преимушество независимости от того, является ли симплициальная сфера $K$ многогранной или нет. Действительно, все известные доказательства необходимости $g$-теоремы для симплициальных многогранников (включая оригинальное доказательство Стенли, приведенное в $\{2.1$, доказательство Макмюллена [59] и недавнее доказательство В. А. Тиморина [86]) следуют одной и той же схеме. А именно, числа $h_{i}, i=1, \ldots, n$, интерпретируются как размерности градуированных компонент $A^{i}$ некоторой алгебры $A$, удовлетворяющей сильной теореме Лефшеца. Последнее утверждение означает, что сушествует элемент $\omega \in A^{1}$ такой, что умножение на $\omega$ определяет мономорфизм $A^{i} \rightarrow A^{i+1}$ при $i<\left[\frac{n}{2}\right]$. Отсюда выводится, что $h_{i} \leqslant h_{i+1}$ при $i<\left[\frac{n}{2}\right]$ (см. §2.1). Однако такой элемент $\omega$ отсутствует для симплициальных сфер $K$, не являюшихся многогранными сферами. Это 
означает, что для доказательства $g$-гипотезы для симплициальных сфер необходимо выработать принципиально новую технику.

Как уже отмечалось в $\S 1.3$, симплициальные сферы являются горенштейновыми* комплексами. Используя теоремы $1.4 .12,1.4 .13$ и нашу теорему 4.2 .1 , мы получаем следуюшее решение аналога проблемы 3.2.3.

ТЕОРема 4.3.7. Комплекс $\mathscr{Z}_{K}$ является комплексом Пуанкаре (над k) тогда и только тогда, когда для любого симплекса $I \in K$ (включая $I=\varnothing)$ подкомплекс link I имеет гомологии как у сферы размерности $\operatorname{dim}(\operatorname{link} I)$.

$\S$ 4.4. Фактор-пространства многообразия $\mathscr{Z}_{P}$ по свободным действиям тора. Здесь мы возврашаемся к случаю многогранной сферы $K$ (т.е. $K=K_{P}$ ) и изучаем фактор-пространства $\mathscr{Z}_{P}$ по свободно действуюшим подгруппам $H \subset T^{m}$.

Для любого комбинаторного простого многогранника $P^{n}$ обозначим через $s\left(P^{n}\right)$ максимальную размерность подгрупш $H \subset T^{m}$, которые действуют на $\mathscr{Z}_{P}$ свободно. Число $s\left(P^{n}\right)$, очевидно, является комбинаторньм инвариантом многогранника $P^{n}$.

Проблема 4.4 .1 (В.М. Бухштабер). Выразить $s\left(P^{n}\right)$ через известные комбинаторные инварианты многогранника $P^{n}$.

ПРЕДЛОЖЕНИЕ 4.4.2. Если $P^{n}$ имеет $m$ гиперграней, то $s\left(P^{n}\right) \leqslant m-n$.

ДоказАТЕльство. Каждая подгруппа размерности $>m-n$ в торе $T^{m}$ нетривиально пересекается с любой $n$-мерной стационарной подгруппой и поэтому не может действовать на $\mathscr{Z}_{P}$ свободно.

ПРЕДЛОЖЕНИЕ 4.4.3. Диагональная одномерная подгруппа

$$
S_{d}:=\left\{\left(e^{2 \pi i \varphi}, \ldots, e^{2 \pi i \varphi}\right) \in T^{m}\right\}, \quad \varphi \in \mathbb{R}
$$

действует на любом $\mathscr{Z}_{P}$ свободно. Поэтому $s\left(P^{n}\right) \geqslant 1$.

ДокАЗАТЕльство. Так как любая стационарная подгруппа для $\mathscr{Z}_{P}$ является координатной подгруппой (см. определение 3.1.1), она пересекается с $S_{d}$ лишь по единице.

Другая нижняя оценка числа $s\left(P^{n}\right)$ было предложена в работе [48]. Пусть $\mathscr{F}=$ $\left\{F_{1}, \ldots, F_{m}\right\}$ - множество гиперграней многогранника $P^{n}$. Сюръективное отображение $\varrho: \mathscr{F} \rightarrow[k]$ (где $[k]=\{1, \ldots, k\}$ ) называется правильной $k$-цветной раскраской гиперграней $P^{n}$, если $F_{i} \cap F_{j} \neq \varnothing$ влечет $\varrho\left(F_{i}\right) \neq \varrho\left(F_{j}\right)$. Хроматическим числом $\gamma\left(P^{n}\right)$ многогранника $P^{n}$ называется наименьшее $k$, для которого сушествует правильная $k$-цветная раскраска гиперграней $P^{n}$.

ПримеР 4.4.4. Если $P^{n}-2$-смежностный простой многогранник с $m$ гипергранями, то $\gamma\left(P^{n}\right)=m$.

ПРЕДЛОЖЕНИЕ 4.4 .5 [48]. Имеет место неравенство

$$
s\left(P^{n}\right) \geqslant m-\varrho\left(P^{n}\right) .
$$


ДокАЗАТЕЛьСтво. Отображение $\varrho: \mathscr{F} \rightarrow[k]$ определяет эпиморфизм торов $\tilde{\varrho}: T^{m} \rightarrow T^{k}$. Легко видеть, что если $\varrho$ является правильной раскраской, то ker $\tilde{\varrho} \cong T^{m-k}$ свободно действует на $\mathscr{Z}_{P}$.

Пусть $H \subset T^{m}$ - подгруппа размерности $r \leqslant m-n$. Выбрав базис в $H$, мы можем записать ее в виде

$$
H=\left\{\left(e^{2 \pi i\left(s_{11} \varphi_{1}+\cdots+s_{1 r} \varphi_{r}\right)}, \ldots, e^{2 \pi i\left(s_{m 1} \varphi_{1}+\cdots+s_{m r} \varphi_{r}\right)}\right) \in T^{m}\right\},
$$

где $\varphi_{i} \in \mathbb{R}, i=1, \ldots, r$. Целочисленная матрица $S=\left(s_{i j}\right)$ размера $m \times r$ определяет мономорфизм $\mathbb{Z}^{r} \rightarrow \mathbb{Z}^{m}$ на прямое слагаемое. Для каждого подмножества $\left\{i_{1}, \ldots, i_{n}\right\} \subset[m]$ обозначим через $S_{\widehat{i}_{1}, \ldots, \widehat{i}_{n}}$ подматрицу размера $(m-n) \times r$ матришы $S$, получаемую удалением строк $i_{1}, \ldots, i_{n}$. Напомним, что любая вершина $v \in P^{n}$ есть пересечение $n$ гиперграней (см. (30)). Имеет место следующий критерий свободности действия $H$ на $\mathscr{Z}_{P}$.

Лемма 4.4.6. Подгруппа (62) действует свободно на $\mathscr{Z}_{P}$ тогда и только тогда, когда для любой вериины $v=F_{i_{1}} \cap \cdots \cap F_{i_{n}}$ многогранника $P^{n}$ подматрица $S_{\widehat{i}_{1}, \ldots, \widehat{i}_{n}}$ размера $(m-n) \times r$ определяет мономорфизм $\mathbb{Z}^{r} \rightarrow \mathbb{Z}^{m-n}$ на прямое слагаемое.

ДокАЗАТЕЛЬСТво. Как вытекает из определения 3.1 .1 , орбиты $T^{m}$-действия на $\mathscr{Z}_{P}$, соответствуюшие вершинам $P^{n}$, имеют максимальные стационарные подгруппы (ранга $n$ ). Стационарная подгруппа, соответствуюшая вершине $v=F_{i_{1}} \cap \cdots \cap F_{i_{n}}$, является координатной подгруппой $T_{i_{1}, \ldots, i_{n}}^{n} \subset T^{m}$. Подгруппа (62) действует на $\mathscr{Z}_{P}$ свободно тогда и только тогда, когда она пересекает каждую стационарную подгруппу лишш в единице. Это эквивалентно тому, что отображение $H \times T_{i_{1}, \ldots, i_{n}}^{n} \rightarrow T^{m}$ инъективно для любой вершины $v=F_{i_{1}} \cap \cdots \cap F_{i_{n}}$. Такое отображение инъективно, если и только если образ соответствуюшего отображения $\mathbb{Z}^{r+n} \rightarrow \mathbb{Z}^{m}$ является прямым слагаемым в $\mathbb{Z}^{m}$. Матрища этого отображения $\mathbb{Z}^{r+n} \rightarrow \mathbb{Z}^{m}$ получается добавлением $n$ столбцов $(0, \ldots, 0,1,0, \ldots, 0)^{t}$ (1 стоит на месте $\left.i_{j}, j=1, \ldots, n\right)$ к матрице $S$. Эта матриша определяет мономорфизм на прямое слагаемое тогда и только тогда, когда то же самое верно для каждой матрицы $S_{\widehat{i}_{1}, \ldots, \widehat{i}_{n}}$.

В частности, для подгрупп ранга $m-n$ мы получаем

СЛЕДСТВИЕ 4.4.7. Подгруппа (62) ранга $r=m-n$ действует свободно на $\mathscr{Z}_{P}$ тогда и только тогда, когда для любой вершины $v=F_{i_{1}} \cap \cdots \cap F_{i_{n}}$ многогранника $P^{n}$ минор $S_{\widehat{i}_{1}, \ldots, \widehat{i}_{n}}$ удовлетворяет $\operatorname{det} S_{\widehat{i}_{1} \ldots \widehat{i}_{n}}= \pm 1$.

ПрЕДЛОЖЕНИЕ 4.4.8. Простой многогранник $P^{n}$ допускает характеристическое отображение тогда и только тогда, когда $s\left(P^{n}\right)=m-n$.

ДоказАТЕльство. Предложение 3.1.5 показывает, что если $P^{n}$ допускает характеристическое отображение $\ell$, то $(m-n)$-мерная подгруппа $H(\ell)$ действует свободно на $\mathscr{Z}_{P}$, откуда $s\left(P^{n}\right)=m-n$. Пусть теперь $s\left(P^{n}\right)=m-n$, т.е. сушествует подгруппа (62) ранга $r=m-n$, которая свободно действует на $\mathscr{Z}_{P}$. Соответствуюшая $(m \times(m-n))$-матрица $S$ определяет мономорфизм $\mathbb{Z}^{m-n} \rightarrow \mathbb{Z}^{m}$ на прямое слагаемое. Тогда сушествует $(n \times m)$-матрица $\Lambda$ такая, что последовательность

$$
0 \longrightarrow \mathbb{Z}^{m-n} \stackrel{S}{\longrightarrow} \mathbb{Z}^{m} \stackrel{\Lambda}{\longrightarrow} \mathbb{Z}^{n} \longrightarrow 0
$$


является точной. Так как $S$ удовлетворяет условию из следствия 4.4.7, матрица $\Lambda$ удовлетворяет условию (26) и, таким образом, определяет характеристическое отображение для $P^{n}$.

Для каждой подгрупшы (62) ранга $r=m-n$ определим следующие линейные формы в $\mathbf{k}\left[v_{1}, \ldots, v_{m}\right]$ :

$$
w_{i}=s_{1 i} v_{1}+\cdots+s_{m i} v_{m}, \quad i=1, \ldots, m-n .
$$

Пусть $M^{2 n}$ - квазиторическое многообразиенад $P^{n}$ с характеристическим отображением $\ell$. Запишем подгруппу $H(\ell)$ в виде (62); это определяет элементы (63). При этих предположениях имеет место следующее утверждение.

ЛЕмма 4.4.9. Имеет место следующий изоморфизм алгебр:

$$
H^{*}\left(\mathscr{Z}_{P}\right) \cong \operatorname{Tor}_{\mathbf{k}\left[w_{1}, \ldots, w_{m-n}\right]}\left(H^{*}\left(M^{2 n}\right), \mathbf{k}\right) \text {, }
$$

где структура $\mathbf{k}\left[w_{1}, \ldots, w_{m-n}\right]$-модуля в $H^{*}\left(M^{2 n}\right)=\mathbf{k}\left[v_{1}, \ldots, v_{m}\right] / \mathscr{I}_{P}+\mathscr{J}_{\ell}$ задается при помощи формул (63).

ДокАЗАТЕльство. Теорема 4.2.11 показьвает, что

$$
H^{*}\left(\mathscr{Z}_{K}\right) \cong \operatorname{Tor}_{\mathbf{k}\left[v_{1}, \ldots, v_{m}\right] / \mathscr{J}_{\ell}}\left(\mathbf{k}(K) / \mathscr{J}_{\ell}, \mathbf{k}\right) \text {. }
$$

Фактор-кольцо $\mathbf{k}\left[v_{1}, \ldots, v_{m}\right] / \mathscr{J}_{\ell}$ отождествляется с $\mathbf{k}\left[w_{1}, \ldots, w_{m-n}\right]$.

Теорема 4.4.10. Спектральная последовательность Лере-Серра $T^{m-n}$-расслоения $\mathscr{Z}_{P} \rightarrow M^{2 n}$ вырождается в члене $E_{3}$. Кроме того, имеет место следующий изоморфизм алгебр:

$$
\begin{gathered}
H^{*}\left(\mathscr{Z}_{P}\right) \cong H\left[\Lambda\left[u_{1}, \ldots, u_{m-n}\right] \otimes\left(\mathbf{k}(P) / \mathscr{J}_{\ell}\right), d\right], \\
\operatorname{bideg} v_{i}=(0,2), \quad \operatorname{bideg} u_{i}=(-1,2) ; \\
d\left(u_{i}\right)=w_{i}, \quad d\left(v_{i}\right)=0 .
\end{gathered}
$$

ДокАЗАТЕЛЬСТво. Так как $H^{*}\left(T^{m-n}\right)=\Lambda\left[u_{1}, \ldots, u_{m-n}\right], H^{*}\left(M^{2 n}\right)=\mathbf{k}(P) / \mathscr{J}_{\ell}$, мы имеем

$$
E_{3} \cong H\left[\left(\mathbf{k}(P) / \mathscr{J}_{\ell}\right) \otimes \Lambda\left[u_{1}, \ldots, u_{m-n}\right], d\right] .
$$

Из леммы 1.4.6 вытекает

$$
H\left[\left(\mathbf{k}(P) / \mathscr{J}_{\ell}\right) \otimes \Lambda\left[u_{1}, \ldots, u_{m-n}\right], d\right] \cong \operatorname{Tor}_{\mathbf{k}\left[w_{1}, \ldots, w_{m-n}\right]}\left(H^{*}\left(M^{2 n}\right), \mathbf{k}\right) .
$$

Сопоставляя два предыдуших тождества с леммой 4.4 .9 , мы получаем $E_{3}=H^{*}\left(\mathscr{Z}_{P}\right)$, что завершает доказательство.

Теперь мы опишем кольцо когомологий фактор-пространства $\mathscr{Z}_{P} / H$ для любой свободно действуюшей подгрупшы $H$. Вначале запишем $H$ в виде $(62)$ и выберем $((m-r) \times m)$-матрицу $T=\left(t_{i j}\right)$ ранга $(m-r)$, удовлетворяюшую $T \cdot S=0$. Это делается таким же образом, как при доказательстве предложения 4.4 .8 (в частности, $T$ - характеристическая матрица квазиторического многообразия $\mathscr{Z}_{P} / H$ в случае $r=m-n)$. 
Теорема 4.4.11. Имеет место следующий изоморфизм алгебр:

$$
H^{*}\left(\mathscr{Z}_{P} / H\right) \cong \operatorname{Tor}_{\mathbf{k}\left[t_{1}, \ldots, t_{m-r}\right]}(\mathbf{k}(P), \mathbf{k}),
$$

где структура $\mathbf{k}\left[t_{1}, \ldots, t_{m-r}\right]$-модуля в $\mathbf{k}(P)=\mathbf{k}\left[v_{1}, \ldots, v_{m}\right] / \mathscr{I}_{P}$ задается отобрахсением

$$
\begin{aligned}
k\left[t_{1}, \ldots, t_{m-r}\right] & \rightarrow k\left[v_{1}, \ldots, v_{m}\right] \\
t_{i} & \rightarrow t_{i 1} v_{1}+\cdots+t_{i m} v_{m}
\end{aligned}
$$

ЗАмечАниЕ. Теорема 4.4 .11 сводится к теореме 4.2 .1 в случае $r=0$ и к примеру 4.1.5 в случае $r=m-n$.

ДокАЗАТЕльСТВо ТЕОРЕмЫ 4.4.11. Вложение подгрупш $T^{r} \cong H \hookrightarrow T^{m}$ определяет отображение классифицирующих пространств $h: B T^{r} \rightarrow B T^{m}$. Рассмотрим коммутативный квадрат

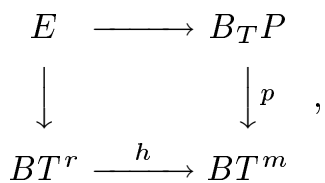

где левая вертикальная стрелка обозначает расслоение, индуцированное отображением $h$. Легко показать, что $E$ гомотопически эквивалентно фактор-пространству $\mathscr{Z}_{P} / H$. Спектральная последовательность Эйленберга-Мура предыдущего коммутативного квадрата сходится к когомологиям $\mathscr{Z}_{P} / H$ и имеет

$$
E_{2}=\operatorname{Tor}_{\mathbf{k}\left[v_{1}, \ldots, v_{m}\right]}\left(\mathbf{k}(P), \mathbf{k}\left[w_{1}, \ldots, w_{r}\right]\right),
$$

где структура $\mathbf{k}\left[v_{1}, \ldots, v_{m}\right]$-модуля в $\mathbf{k}\left[w_{1}, \ldots, w_{r}\right]$ задается матрищей $S$, т.е. отображением $v_{i} \rightarrow s_{i 1} w_{1}+\cdots+s_{i r} w_{r}$. Таким же образом, как и в доказательстве теоремы 4.2.1 (используя клеточные разбиения), показьвается, что спектральная последовательность вырождается в члене $E_{2}$ и имеет место изоморфизм алгебр

$$
H^{*}\left(\mathscr{Z}_{P} / H\right)=\operatorname{Tor}_{\mathbf{k}\left[v_{1}, \ldots, v_{m}\right]}\left(\mathbf{k}(P), \mathbf{k}\left[w_{1}, \ldots, w_{r}\right]\right) .
$$

Положим теперь в теореме 1.4.10

$$
\Lambda=\mathbf{k}\left[v_{1}, \ldots, v_{m}\right], \quad \Gamma=\mathbf{k}\left[t_{1}, \ldots, t_{m-r}\right], \quad A=\mathbf{k}\left[w_{1}, \ldots, w_{r}\right] \quad \text { и } C=\mathbf{k}(P) .
$$

Так как $\Lambda$ является свободным $\Gamma$-модулем и $\Omega=\Lambda / \Gamma=k\left[w_{1}, \ldots, w_{r}\right]$, возникает спектральная последовательность $\left\{\widetilde{E}_{s}, \widetilde{d}_{s}\right\}$. Ее член $E_{2}$ есть

$$
\widetilde{E}_{2}=\operatorname{Tor}_{\mathbf{k}\left[w_{1}, \ldots, w_{r}\right]}\left(\mathbf{k}\left[w_{1}, \ldots, w_{r}\right], \operatorname{Tor}_{\mathbf{k}\left[t_{1}, \ldots, t_{m-r}\right]}(\mathbf{k}(P), \mathbf{k})\right),
$$

и она сходится к $\operatorname{Tor}_{\mathbf{k}\left[v_{1}, \ldots, v_{m}\right]}\left(\mathbf{k}(P), \mathbf{k}\left[w_{1}, \ldots, w_{r}\right]\right)$. Так как $\mathbf{k}\left[w_{1}, \ldots, w_{r}\right]$ является свободным $\mathbf{k}\left[w_{1}, \ldots, w_{r}\right]$-модулем, мы имеем

$$
\widetilde{E}_{2}^{p, q}=0 \text { при } p \neq 0, \quad \widetilde{E}_{2}^{0, q}=\operatorname{Tor}_{\mathbf{k}\left[t_{1}, \ldots, t_{m-r}\right]}^{q}(\mathbf{k}(P), \mathbf{k}) .
$$

Следовательно, спектральная последовательность вырождается в члене $E_{2}$, и имеет место изоморфизм алгебр

$$
\operatorname{Tor}_{\mathbf{k}\left[v_{1}, \ldots, v_{m}\right]}\left(\mathbf{k}(P), \mathbf{k}\left[w_{1}, \ldots, w_{r}\right]\right) \cong \operatorname{Tor}_{\mathbf{k}\left[t_{1}, \ldots, t_{m-r}\right]}(\mathbf{k}(P), \mathbf{k}),
$$

которьй вместе с (64) доказывает теорему. 
СлЕДСТВИЕ 4.4.12. $H^{*}\left(\mathscr{Z}_{P} / H\right) \cong H\left[\Lambda\left[u_{1}, \ldots, u_{m-r}\right] \otimes \mathbf{k}, d\right]$, əде $d u_{i}=\left(t_{i 1} v_{1}+\right.$ $\left.\cdots+t_{i m} v_{m}\right), d v_{i}=0$, bideg $v_{i}=(0,2)$, bideg $u_{i}=(-1,2)$.

ПримеР 4.4.13. Пусть $H=S_{d}$ (см. предложение 4.4.3). В этой ситуации матрица $S$ представляет собой столбец из $m$ единиц. По теореме 4.4.11,

$$
H^{*}\left(\mathscr{Z}_{P} / S_{d}\right) \cong \operatorname{Tor}_{\mathbf{k}\left[t_{1}, \ldots, t_{m-1}\right]}(\mathbf{k}(P), \mathbf{k})
$$

где структура $\mathbf{k}\left[t_{1}, \ldots, t_{m-1}\right]$-модуля в $\mathbf{k}(P)=\mathbf{k}\left[v_{1}, \ldots, v_{m}\right] / I$ определяется отображением

$$
t_{i} \rightarrow v_{i}-v_{m}, \quad i=1, \ldots, m-1 .
$$

$S^{1}$-расслоение $\mathscr{Z}_{P} \rightarrow \mathscr{Z}_{P} / S_{d}$ классифицируется некоторым отображением $c: \mathscr{Z}_{P} / S_{d}$ $\rightarrow B T^{1} \cong \mathbb{C} P^{\infty}$. Так как $H^{*}\left(\mathbb{C} P^{\infty}\right)=\mathbf{k}[w]$, определен элемент $c^{*}(w) \in H^{2}\left(\mathscr{Z}_{P} / S_{d}\right)$.

Лемма 4.4.14. $P^{n}$ является $q$-смежностныц тогда и только тогда, когда $\left(c^{*}(w)\right)^{q} \neq 0$.

ДокАЗАТЕЛЬСТво. Отображение $c^{*}$ переводит кольцо когомологий $H^{*}\left(B T^{1}\right) \cong$ $\mathbf{k}[w]$ в подкольцо $\mathbf{k}(P) \otimes_{\mathbf{k}\left[t_{1}, \ldots, t_{m-1}\right]} \mathbf{k}=\operatorname{Tor}_{\mathbf{k}\left[t_{1}, \ldots, t_{m-1}\right]}^{0}(\mathbf{k}(P), \mathbf{k})$ кольца $H^{*}\left(\mathscr{Z}_{P} / H\right)$. Это подкольцо изоморфно фактор-кольцу $\mathbf{k}(P) /\left(v_{1}=\cdots=v_{m}\right)$. Теперь утверждение вытекает из того факта, что многогранник $P^{n}$ является $q$-смежностным, если и только если идеал $\mathscr{I}_{P}$ не содержит мономов степени $<q+1$.

$\S$ 4.5. Биградуированная двойственность Пуанкаре и аналоги соотношений Дена-Соммервилля для симплициальных многообразий. Здесь мы предполагаем, что $K^{n-1}$ является симплициальным многообразием. В этом случае момент-угол комплекс $\mathscr{Z}_{K}$, вообще говоря, не является многообразием, однако его особенности легко описываются. Действительно, кубический комплекс сc $(K)$ (конструкция 1.5.9) гомеоморфен $\mid$ cone $(K) \mid$, и вершиной конуса является точка $p=(1, \ldots, 1) \in$ $\operatorname{cc}(K) \subset I^{m}$. Пусть $U_{\varepsilon}(p) \subset \mathrm{cc}(K)$ - малая окрестность точки $p$ в $c c(K)$. Тогда замыкание окрестности $U_{\varepsilon}(p)$ также гомеоморфно $|\operatorname{cone}(K)|$. Из определения $\mathscr{Z}_{K}$ (см. (39)) вытекает, что $U_{\varepsilon}\left(T^{m}\right):=\rho^{-1}\left(U_{\varepsilon}(p)\right) \subset \mathscr{Z}_{K}$ - малая инвариантная окрестность тора $T^{m}=\rho^{-1}(p)$ в $\mathscr{Z}_{K}$. Тогда для малых $\varepsilon$ замыкание окрестности $U_{\varepsilon}\left(T^{m}\right)$ гомеоморффно $\mid$ cone $(K) \mid \times T^{m}$. Удаляя $U_{\varepsilon}\left(T^{m}\right)$ из $\mathscr{Z}_{K}$, мы получаем многообразие с границей, которое будем обозначать $W_{K}$. Итак, мы имеем

$$
W_{K}=\mathscr{Z}_{K} \backslash U_{\varepsilon}\left(T^{m}\right), \quad \partial W_{K}=|K| \times T^{m} .
$$

Заметим, что, поскольку окрестность $U_{\varepsilon}\left(T^{m}\right)$ является $T^{m}$-инвариантной, тор $T^{m}$ действует на $W_{K}$.

Tеорема 4.5.1. Многообразие с граничей $W_{K}$ әквивариантно гомотопически әквивалентно момент-угол комплексу $\mathscr{W}_{K}$ (см. (39)). Имеет место канонический относительныи ио гомеоморфизм пар $\left(W_{K}, \partial W_{K}\right) \rightarrow\left(\mathscr{Z}_{K}, T^{m}\right)$. 


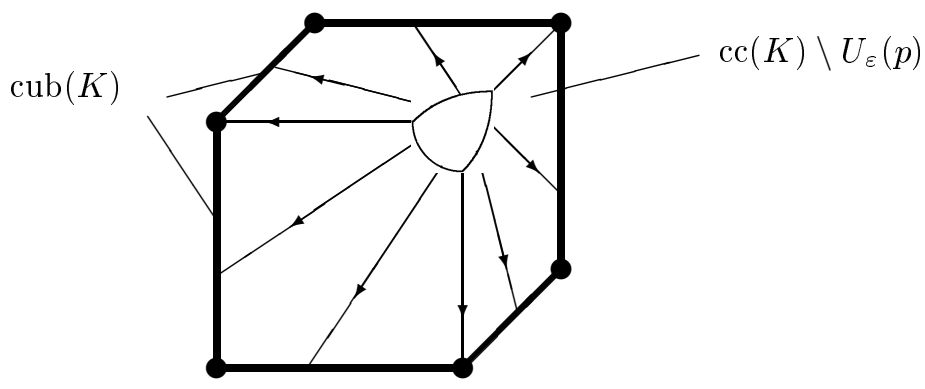

Рис. 9. Гомотопическая эквивалентность сс $(K) \backslash U_{\varepsilon}(p) \rightarrow \operatorname{cub}(K)$

ДокАЗАТЕЛЬСтво. Для доказательства первого утверждения построим гомотопическую эквивалентность сc $(K) \backslash U_{\varepsilon}(p) \rightarrow \operatorname{cub}(K)$, как показано на рис. 9. Это отображение накрывается эквивариантной гомотопической эквивалентностью $W_{K}=$ $\mathscr{Z}_{K} \backslash U_{\varepsilon}\left(T^{m}\right) \rightarrow \mathscr{W}_{K}$. Второе утверждение легко следует из определения $W_{K}$.

Как показывает лемма 3.3.1, момент-угол комплекс $\mathscr{W}_{K} \subset\left(D^{2}\right)^{m}$ имеет клеточную структуру с 5 различньми типами клеток $D_{i}, I_{i}, 0_{i}, T_{i}, 1_{i}, i=1, \ldots, m$ (см. рис. 7). Гомологии комплекса $\mathscr{W}_{K}$ (а значит, и многообразия $W_{K}$ ) можно вычислять при помоши соответствуюшего клеточного цепного комплекса, который мы будем обозначать $\left[\mathscr{C}_{*}\left(\mathscr{W}_{K}\right), \partial_{c}\right]$. Несмотря на то, что $\mathscr{W}_{K}$ имеет больше типов клеток, чем $\mathscr{Z}_{K}$ (напомним, что $\mathscr{Z}_{K}$ имеет лишь три типа клеток $D_{i}, T_{i}, 1_{i}$ ), комплекс клеточных цепей $\left[\mathscr{C}_{*}\left(\mathscr{W}_{K}\right), \partial_{c}\right]$ также имеет каноническую биградуированную структуру. А именно, имеет место следующее утверждение (сравните с (46)).

ЛЕмма 4.5.2. Положим

$$
\begin{gathered}
\operatorname{bideg} D_{i}=(0,2), \quad \operatorname{bideg} T_{i}=(-1,2), \quad \operatorname{bideg} I_{i}=(1,0), \\
\operatorname{bideg} 0_{i}=\operatorname{bideg} 1_{i}=(0,0), \quad i=1, \ldots, m .
\end{gathered}
$$

Это превращает клеточный чепной комплекс $\left[\mathscr{C}_{*}\left(\mathscr{W}_{K}\right), \partial_{c}\right]$ в биградуированный дифференциальныи модуль с дифференциалом $\partial_{c}$, уменьшающим бистепень на $(1,0)$. Исходная градуировка в $\mathscr{C}_{*}\left(\mathscr{W}_{K}\right)$ по размерностям клеток соответствует полной степени (т.е. размерность клетки есть сумма ее двух степеней).

ДокАЗАТЕЛЬСтво. Необходимо лишш проверить, что дифференциал $\partial_{c}$ уменњшает бистепень на $(1,0)$. Это вытекает из $(66)$ и

$$
\partial_{c} D_{i}=T_{i}, \quad \partial_{c} I_{i}=1_{i}-0_{i}, \quad \partial_{c} T_{i}=\partial_{c} 1_{i}=\partial_{c} 0_{i}=0 .
$$

Заметим, что, в отличие от биградуированной структуры в $\mathscr{C}_{*}\left(\mathscr{Z}_{K}\right)$, элементы комплекса $\mathscr{C}_{*, *}\left(\mathscr{W}_{K}\right)$ могут иметь положительную первую градуировку (из-за положительной первой градуировки клетки $\left.I_{i}\right)$. При этом дифференциал $\partial_{c}$ не меняет второй градуировки (как и в случае $\mathscr{Z}_{K}$ ), что позволяет представить биградуированњый комплекс $\mathscr{C}_{*, *}\left(\mathscr{W}_{K}\right)$ в виде суммы комплексов $\mathscr{C}_{*, 2 p}\left(\mathscr{W}_{K}\right), p=0, \ldots, m$. 
Аналогично тому, как мы это делали для комплекса $\mathscr{Z}_{K}$ и для пары $\left(\mathscr{Z}_{K}, T^{m}\right)$, введем

$$
\begin{gathered}
b_{q, 2 p}\left(\mathscr{W}_{K}\right)=\operatorname{dim} H_{q, 2 p}\left[\mathscr{C}_{*, *}\left(\mathscr{W}_{K}\right), \partial_{c}\right], \quad-m \leqslant q \leqslant m, \quad 0 \leqslant p \leqslant m ; \\
\chi_{p}\left(\mathscr{W}_{K}\right)=\sum_{q=-m}^{m}(-1)^{q} \operatorname{dim} \mathscr{C}_{q, 2 p}\left(\mathscr{W}_{K}\right)=\sum_{q=-m}^{m}(-1)^{q} b_{q, 2 p}\left(\mathscr{W}_{K}\right) ; \\
\chi\left(\mathscr{W}_{K} ; t\right)=\sum_{p=0}^{m} \chi_{p}\left(\mathscr{W}_{K}\right) t^{2 p}
\end{gathered}
$$

(заметим, что $q$ здесь может быть как положительным, так и отрицательньм).

Следующая теорема дает точную формулу для порождающего многочлена $\chi\left(\mathscr{W}_{K} ; t\right)$ и аналогична теоремам 4.2 .8 и 4.2 .10 .

ТЕОРема 4.5.3. Для любого симплициального комплекса $K^{n-1}$ с $m$ вершинами имеет место соотношение

$$
\begin{aligned}
\chi\left(\mathscr{W}_{K} ; t\right) & =\left(1-t^{2}\right)^{m-n}\left(h_{0}+h_{1} t^{2}+\cdots+h_{n} t^{2 n}\right)+(\chi(K)-1)\left(1-t^{2}\right)^{m} \\
& =\left(1-t^{2}\right)^{m-n}\left(h_{0}+h_{1} t^{2}+\cdots+h_{n} t^{2 n}\right)+(-1)^{n-1} h_{n}\left(1-t^{2}\right)^{m},
\end{aligned}
$$

əде $\chi(K)=f_{0}-f_{1}+\cdots+(-1)^{n-1} f_{n-1}=1+(-1)^{n-1} h_{n}-$ эйлерова характеристика комплекса $K$.

ДокАЗАТЕЛЬСТво. Из определения $\mathscr{W}_{K}$ (см. (39)) следует, что $D_{I} I_{J} 0_{L} T_{P} 1_{Q}$ (см. $\S 3.3)$ является клеткой в $\mathscr{W}_{K}$ тогда и только тогда, когда выполнены следующие два условия:

(а) множество $I \cup J \cup L$ является симплексом комплекса $K^{n-1}$;

(б) $\# L \geqslant 1$.

Пусть $c_{i j l p q}\left(\mathscr{W}_{K}\right)$ обозначает число клеток $D_{I} I_{J} 0_{L} T_{P} 1_{Q} \subset \mathscr{W}_{K} \mathrm{c} i=\# I, j=\# J$, $l=\# L, p=\# P, q=\# Q, i+j+l+p+q=m$. Тогда

$$
c_{i j l p q}\left(\mathscr{W}_{K}\right)=f_{i+j+l-1}\left(\begin{array}{c}
i+j+l \\
i
\end{array}\right)\left(\begin{array}{c}
j+l \\
l
\end{array}\right)\left(\begin{array}{c}
m-i-j-l \\
p
\end{array}\right),
$$

где $\left(f_{0}, \ldots, f_{n-1}\right)-f$-вектор комплекса $K$ (мы полагаем $f_{-1}=1$ и $f_{k}=0$ при $k<-1$ или $k>n-1)$. В силу (66)

$$
\operatorname{bideg}\left(D_{I} I_{J} 0_{L} T_{P} 1_{Q}\right)=(j-p, 2(i+p)) .
$$

Вычислим $\chi_{r}\left(\mathscr{W}_{K}\right)$, используя (68) и (69):

$$
\chi_{r}\left(\mathscr{W}_{K}\right)=\sum_{\substack{i, j, l, p \\
i+p=r, l \geqslant 1}}(-1)^{j-p} f_{i+j+l-1}\left(\begin{array}{c}
i+j+l \\
i
\end{array}\right)\left(\begin{array}{c}
j+l \\
l
\end{array}\right)\left(\begin{array}{c}
m-i-j-l \\
p
\end{array}\right) .
$$

Подставляя $s=i+j+l$ в предыдущей формуле, получаем

$$
\begin{aligned}
\chi_{r}\left(\mathscr{W}_{K}\right) & =\sum_{\substack{l, s, p \\
l \geqslant 1}}(-1)^{s-r-l} f_{s-1}\left(\begin{array}{c}
s \\
r-p
\end{array}\right)\left(\begin{array}{c}
s-r+p \\
l
\end{array}\right)\left(\begin{array}{c}
m-s \\
p
\end{array}\right) \\
& =\sum_{s, p}\left((-1)^{s-r} f_{s-1}\left(\begin{array}{c}
s \\
r-p
\end{array}\right)\left(\begin{array}{c}
m-s \\
p
\end{array}\right) \sum_{l \geqslant 1}(-1)^{l}\left(\begin{array}{c}
s-r+p \\
l
\end{array}\right)\right) .
\end{aligned}
$$


Так как

$$
\sum_{l \geqslant 1}(-1)^{l}\left(\begin{array}{c}
s-r+p \\
l
\end{array}\right)=\left\{\begin{aligned}
-1, & s>r-p \\
0, & s \leqslant r-p
\end{aligned}\right.
$$

мы имеем

$$
\begin{aligned}
\chi_{r}\left(\mathscr{W}_{K}\right) & =-\sum_{\substack{s, p \\
s>r-p}}(-1)^{s-r} f_{s-1}\left(\begin{array}{c}
s \\
r-p
\end{array}\right)\left(\begin{array}{c}
m-s \\
p
\end{array}\right) \\
& =-\sum_{s, p}(-1)^{r-s} f_{s-1}\left(\begin{array}{c}
s \\
r-p
\end{array}\right)\left(\begin{array}{c}
m-s \\
p
\end{array}\right)+\sum_{s}(-1)^{r-s} f_{s-1}\left(\begin{array}{c}
m-s \\
r-s
\end{array}\right) .
\end{aligned}
$$

Вторая сумма в последней формуле есть в точности $\chi_{r}\left(\mathscr{Z}_{K}\right)$ (см. (53)). Для того чтобы вычислить первую сумму, заметим, что $\sum_{p}\left(\begin{array}{c}s \\ r-p\end{array}\right)\left(\begin{array}{c}m-s \\ p\end{array}\right)=\left(\begin{array}{c}m \\ r\end{array}\right)$ (это получается при вычислении коэффишиента при $\alpha^{r}$ в обеих частях тождества $(1+\alpha)^{s} \times$ $\left.(1+\alpha)^{m-s}=(1+\alpha)^{m}\right)$. Следовательно,

$$
\chi_{r}\left(\mathscr{W}_{K}\right)=-\sum_{s}(-1)^{r-s} f_{s-1}\left(\begin{array}{c}
m \\
r
\end{array}\right)+\chi_{r}\left(\mathscr{Z}_{K}\right)=(-1)^{r}\left(\begin{array}{c}
m \\
r
\end{array}\right)(\chi(K)-1)+\chi_{r}\left(\mathscr{Z}_{K}\right)
$$

так как $-\sum_{s}(-1)^{s} f_{s-1}=\chi(K)-1$ (напомним, что $f_{-1}=1$ ). Наконец, используя (52), мы находим

$$
\begin{aligned}
\chi\left(\mathscr{W}_{K} ; t\right) & =\sum_{r=0}^{m} \chi_{r}\left(\mathscr{W}_{K}\right) t^{2 r}=\sum_{r=0}^{m}(-1)^{r}\left(\begin{array}{c}
m \\
r
\end{array}\right)(\chi(K)-1) t^{2 r}+\sum_{r=0}^{m} \chi_{r}\left(\mathscr{Z}_{K}\right) t^{2 r} \\
& =(\chi(K)-1)\left(1-t^{2}\right)^{m}+\left(1-t^{2}\right)^{m-n}\left(h_{0}+h_{1} t^{2}+\cdots+h_{n} t^{2 n}\right) .
\end{aligned}
$$

Предположим, что $K$ является ориентируемым симплищиальным многообразием. Тогда легко видеть, что $W_{K}$ также ориентируемо. Таким образом, имеют место изоморфизмы относительной двойственности Пуанкаре:

$$
H_{k}\left(W_{K}\right) \cong H^{m+n-k}\left(W_{K}, \partial W_{K}\right), \quad k=0, \ldots, m
$$

СлЕДСтвиЕ 4.5.4 (обобшенные соотношения Дена-Соммервилля). Следующие соотношения имеют место для $h$-вектора $\left(h_{0}, h_{1}, \ldots, h_{n}\right)$ ориентируемого симплициального многообразия $K^{n-1}$ :

$$
h_{n-i}-h_{i}=(-1)^{i}\left(\chi\left(K^{n-1}\right)-\chi\left(S^{n-1}\right)\right)\left(\begin{array}{c}
n \\
i
\end{array}\right), \quad i=0,1, \ldots, n
$$

где $\chi\left(S^{n-1}\right)=1+(-1)^{n-1}$ - эйлерова характеристика $(n-1)$-сферы. 
ДокАЗАТЕЛЬСтво. Как вытекает из теоремы 4.5.1, $H^{m+n-k}\left(W_{K}, \partial_{c} W_{K}\right)=$ $H^{m+n-k}\left(\mathscr{Z}_{K}, T^{m}\right)$ и $H_{k}\left(W_{K}\right)=H_{k}\left(\mathscr{W}_{K}\right)$. Более того, можно показать таким же образом, как и следствии 4.3.2, что изоморфизмы (70) сохраняют биградуированную структуру в (ко)гомологиях комплекса $\mathscr{W}_{K}$ и пары $\left(\mathscr{Z}_{K}, T^{m}\right)$. Тогда имеем

$$
\begin{aligned}
b_{-q, 2 p}\left(\mathscr{W}_{K}\right) & =b_{-(m-n)+q, 2(m-p)}\left(\mathscr{Z}_{K}, T^{m}\right), \\
\chi_{p}\left(\mathscr{W}_{K}\right) & =(-1)^{m-n} \chi_{m-p}\left(\mathscr{Z}_{K}, T^{m}\right), \\
\chi\left(\mathscr{W}_{K} ; t\right) & =(-1)^{m-n} t^{2 m} \chi\left(\mathscr{Z}_{K}, T^{m} ; \frac{1}{t}\right) .
\end{aligned}
$$

Используя (58), находим

$$
\begin{aligned}
(-1)^{m-n} t^{2 m} \chi\left(\mathscr{Z}_{K}, T^{m} ; \frac{1}{t}\right) & \\
= & (-1)^{m-n} t^{2 m}\left(1-t^{-2}\right)^{m-n}\left(h_{0}+h_{1} t^{-2}+\cdots+h_{n} t^{-2 n}\right) \\
& \quad-(-1)^{m-n} t^{2 m}\left(1-t^{-2}\right)^{m} \\
= & \left(1-t^{2}\right)^{m-n}\left(h_{0} t^{2 n}+h_{1} t^{2 n-2}+\cdots+h_{n}\right)+(-1)^{n-1}\left(1-t^{2}\right)^{m} .
\end{aligned}
$$

Подставляя формулу для $\chi\left(\mathscr{W}_{K} ; t\right)$ из теоремы 4.5.3 и предыдущее выражение в $(71)$, получаем

$$
\begin{aligned}
\left(1-t^{2}\right)^{m-n} & \left(h_{0}+h_{1} t^{2}+\cdots+h_{n} t^{2 n}\right)+(\chi(K)-1)\left(1-t^{2}\right)^{m} \\
& =\left(1-t^{2}\right)^{m-n}\left(h_{0} t^{2 n}+h_{1} t^{2 n-2}+\cdots+h_{n}\right)+(-1)^{n-1}\left(1-t^{2}\right)^{m} .
\end{aligned}
$$

Разделив это соотношение на $\left(1-t^{2}\right)^{m-n}$ и вычисляя коэффициент при $t^{2 i}$ в обеих его частях, мы получаем $h_{n-i}-h_{i}=(-1)^{i}\left(\chi\left(K^{n-1}\right)-\chi\left(S^{n-1}\right)\right)\left(\begin{array}{c}n \\ i\end{array}\right)$, что и требовалось.

Если $|K|=S^{n-1}$ или $(n-1)$ нечетно, следствие 4.5.4 дает классические соотношения $h_{n-i}=h_{i}$.

СлЕДСТВИЕ 4.5.5. Если $K^{n-1}$ - симплициальное многообразие с $h$-вектором $\left(h_{0}, \ldots, h_{n}\right)$, mo

$$
h_{n-i}-h_{i}=(-1)^{i}\left(h_{n}-1\right)\left(\begin{array}{c}
n \\
i
\end{array}\right), \quad i=0,1, \ldots, n .
$$

ДоКАЗАТЕЛЬСТво. Так как $\chi\left(K^{n-1}\right)=1+(-1)^{n-1} h_{n}, \chi\left(S^{n-1}\right)=1+(-1)^{n-1}$, мы имеем

$$
\chi\left(K^{n-1}\right)-\chi\left(S^{n-1}\right)=(-1)^{n-1}\left(h_{n}-1\right)=\left(h_{n}-1\right)
$$

(коэффициент $(-1)^{n-1}$ может быть опушен, так как для нечетных $(n-1)$ левая часть обрашается в нуль).

СЛЕДСТВИЕ 4.5.6. Для любого $(n-1)$-мерного ориентируемого симплищиального многообразия числа $h_{n-i}-h_{i}, i=0,1, \ldots, n$, являются гомотопическими инвариантами. В частности, они не зависят от триангуляиии.

В частном случае $P L$-многообразий топологическая инвариантность чисел $h_{n-i}-h_{i}$ была впервые наблюдена Пахнером в $[68 ;(7.11)]$.

ПримеР 4.5.7. Рассмотрим триангулящии двумерного тора $T^{2}$. Мы имеем $n=3$, $\chi\left(T^{2}\right)=0$. Из $\chi\left(K^{n-1}\right)=1+(-1)^{n-1} h_{n}$ мы получаем $h_{3}=-1$. Следствие 4.5 .4 дает

$$
h_{3}-h_{0}=-2, \quad h_{2}-h_{1}=6 .
$$

Например, триангулящия на рис. 10 имеет $f_{0}=9$ вершин, $f_{1}=27$ ребер и $f_{2}=18$ треугольников. Соответствуюший $h$-вектор есть $(1,6,12,-1)$. 


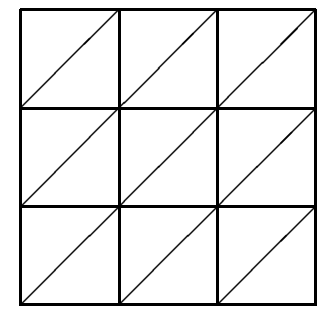

Рис. 10. Триангуляция тора $T^{2}$ с $\boldsymbol{f}=(9,27,18), \boldsymbol{h}=(1,6,12,-1)$

\section{Глава 5. Конфигурации подпространств и кольца когомологий их дополнений}

§5.1. Обзор результатов о когомологиях дополнений к общим конфигурациям. Конфигурачией называется конечное множество $\mathscr{A}=\left\{L_{1}, \ldots, L_{r}\right\}$ плоскостей (аффинных подпространств) в некотором аффинном пространстве (вешественном или комплексном). Для каждой конфигурации $\mathscr{A}=\left\{L_{1}, \ldots, L_{r}\right\}$ в $\mathbb{C}^{m}$ определим ее носитель $|\mathscr{A}|$ как

$$
|\mathscr{A}|:=\bigcup_{i=1}^{r} L_{i} \subset \mathbb{C}^{m}
$$

и дополнение $U(\mathscr{A})$ как

$$
U(\mathscr{A}):=\mathbb{C}^{m} \backslash|\mathscr{A}|,
$$

и аналогично для конфигураций в $\mathbb{R}^{m}$.

Конфигурации и их дополнения играют ключевую роль во многих конструкциях комбинаторики, алгебраической и симплектической геометрии и т. д.; они также возникают как конфигурационные пространства для различных классических механических систем. При изучении конфигураций весьма важно иметь достаточно детальное описание топологии дополнений $U(\mathscr{A})$ (оно включает число связных компонент, гомотопический тип, группы гомологий, кольца когомологий и т. д.). Множество замечательных результатов в этом направлении было получено в течение последних трех десятилетий, однако общая картина далека от завершения. Теория берет начало от работы Арнольда [4], в которой классифицируюшее пространство для группы кос $B_{n}$ описано как дополнение конфигурации диагональных гиперплоскостей $\left\{z_{i}=z_{j}\right\}$ в $\mathbb{C}^{n}$. Там же было вычислено кольцо когомологий дополнения этой конфигурации. Этот результат был обобшен Брискорном [18] и мотивировал дальнейшее развитие теории конфигураций комплексных гиперплоскостей (т.е. конфигураций комплексных аф̆финных подпространств коразмерности один). Одним из основных результатов здесь является следуюшая теорема.

Teорема 5.1.1 [4], [18], [65]. Пусть $\mathscr{A}=\left\{L_{1}, \ldots, L_{r}\right\}$ - конфигурачия комплексных әиперплоскостей в $\mathbb{C}^{m}$, причем гиперплоскость $L_{i}$ задается как множество нулей линейной функиии $l_{i}, i=1, \ldots, r$. Тогда алгебра челочисленных когомологий дополнения $\mathbb{C}^{m} \backslash|\mathscr{A}|$ изоморфна алгебре, порожденной замкнутыми дифференциальныцми 1-фор.мами $\frac{1}{2 \pi i} \frac{d l_{i}}{l_{i}}$. 
Соотношения между формами $\frac{1}{2 \pi i} \frac{d l_{i}}{l_{i}}$ явно описаны. В случае конфигурации диагональных гиперплоскостей $\left\{z_{i}=z_{j}\right\}$ мы имеем формы $\omega_{i j}=\frac{1}{2 \pi i} \frac{d\left(z_{i}-z_{j}\right)}{z_{i}-z_{j}}$ и соот-
ношения

$$
\omega_{i j} \wedge \omega_{j k}+\omega_{j k} \wedge \omega_{k i}+\omega_{k i} \wedge \omega_{i j}=0 .
$$

Теория конфигураций комплексных гиперплоскостей, возможно, является наиболее разработанной областью общей теории конфигураций. По ней уже выпущено несколько монографий; мы упомянем лиш [66], где можно найти дальнейшие ссылки. Взаимосвязи между конфигурациями вещественных гиперплоскостей, многогранниками и ориентированными матроидами обсуждаются в [89; лекция 7].

В общей ситуации теорема Горески-Макферсона [42; ч. III] выражает группы когомологий $H^{i}(U(\mathscr{A}))$ (без кольцевой структуры) как сумму групп гомологий подкомплексов некоторого симплициального комплекса. Мы приводим этот результат ниже. Обзор результатов об обших конфигурациях плоскостей содержится в работе [14]. Ряд ключевых вопросов в этом направлении изложен в монографии [87].

Пусть $\mathscr{A}=\left\{L_{1}, \ldots, L_{r}\right\}$ - конфигурация плоскостей в $\mathbb{R}^{n}$. Пересечения

$$
v=L_{i_{1}} \cap \cdots \cap L_{i_{k}}
$$

образуют частично упорядоченное множество $(\mathscr{P},<)$ по отношению к включению (т.е. $v<w$ тогда и только тогда, когда $v$ и $w$ различны и $v$ содержится в $w$ ). Мы предполагаем, что частично упорядоченное множество $\mathscr{P}$ содержит единственный максимальньй элемент $T$, соответствующий объемлющему пространству конфигурации. Функиия ранга $d$ на $\mathscr{P}$ определяется как $d(v)=\operatorname{dim} v$. Порядковьй комплекс $K(\mathscr{P})$ (пример 1.2.7) называется порядковым комплексом конфигурачии А. Положим

$$
\mathscr{P}_{(v, w)}=\{x \in \mathscr{P}: v<x<w\}, \quad \mathscr{P}_{>v}=\{x \in \mathscr{P}: x>v\} .
$$

Теорема 5.1.2 (Горески, Макферсон [42; ч. III]). Имеет место следующая формула для гомологий дополнения $U(\mathscr{A})$ :

$$
H_{i}(U(\mathscr{A}) ; \mathbb{Z})=\bigoplus_{v \in \mathscr{P}} H^{n-d(v)-i-1}\left(K\left(\mathscr{P}_{>v}\right), K\left(\mathscr{P}_{(v, T)}\right) ; \mathbb{Z}\right)
$$

где принято соглашение $H^{-1}(\varnothing, \varnothing)=\mathbb{Z}$.

Доказательство этой теоремы использует стратифицированную теорию Морса, разработанную в [42].

ЗАмечАниЕ. Группы гомологий комплексной конфигурации в $\mathbb{C}^{n}$ можно вычислять, рассматривая ее как вешественную конфигурацию в $\mathbb{R}^{2 n}$.

Кольи а когомологий дополнений конфигураций изучены значительно хуже. Вообще говоря, кольцо целочисленных когомологий дополнения $U(\mathscr{A})$ не определяется частично упорядоченным множеством $\mathscr{P}$. Один из подходов к вычислению алгебры когомологий дополнения $U(\mathscr{A})$ был предложен Де Кончини и Прочези [34]. В частности, они доказали, что кольцо рациональных когомологий дополнения $U(\mathscr{A})$ определяется комбинаторикой пересечений. Этот результат был развит Юзвинским в [88]. 
§5.2. Конфигурации координатных подпространств и когомологии комплекса $\mathscr{Z}_{K}$. Конфигурация $\mathscr{A}=\left\{L_{1}, \ldots, L_{r}\right\}$ назьвается координатной, если все плоскости $L_{i}, i=1, \ldots, r$, являются координатными подпространствами. В этом параграфе мы применяем результаты главы 4 к вычислению алгебр когомологий дополнений к конфигурациям комплексных координатных подпространств. Случай вешественных координатных конфигураций обсуждается в конце параграфа.

Любое координатное подпространство в $\mathbb{C}^{m}$ имеет вид

$$
L_{I}=\left\{\left(z_{1}, \ldots, z_{m}\right) \in \mathbb{C}^{m}: z_{i_{1}}=\cdots=z_{i_{k}}=0\right\},
$$

где $I=\left\{i_{1}, \ldots, i_{k}\right\}$ - некоторое подмножество в $[m]$. Очевидно, $\operatorname{dim} L_{I}=m-\# I$.

Конструкция 5.2.1. Для любого симплициального комплекса $K$ на множестве $[m]$ введем комплексную координатную конфигурацию $\mathscr{C} \mathscr{A}(K)$ как множество подпространств $L_{I}$ таких, что $I$ не является симплексом в $K$ :

$$
\mathscr{C} \mathscr{A}(K)=\left\{L_{I}: I \notin K\right\}
$$

Обозначим дополнение конфигурации $\mathscr{C} \mathscr{A}(K)$ через $U(K)$, т.е.

$$
U(K)=\mathbb{C}^{m} \backslash \bigcup_{I \notin K} L_{I}
$$

Аналогичным образом определяется вещественная координатная конфигурация $U_{\mathbb{R}}(K)$. Если $K^{\prime} \subset K$ - подкомплекс, то $U\left(K^{\prime}\right) \subset U(K)$.

ПрЕДЛОЖЕНИЕ 5.2.2. Сопоставление $K \mapsto U(K)$ определяет сохраняющее порядок взаимно однозначное соответствие между симплициальными комплексами на множестве $[\mathrm{m}]$ и дополнениями конфигураций координатных подпространств в $\mathbb{C}^{m}$.

ДокАЗАТЕЛЬСТво. Пусть $\mathscr{C} \mathscr{A}$ - конфигурация координатных подпространств в $\mathbb{C}^{m}$. Определим

$$
K(\mathscr{C} \mathscr{A}):=\left\{I \subset[m]: L_{I} \not \subset|\mathscr{C} \mathscr{A}|\right\} .
$$

Легко видеть, что $K(\mathscr{C} \mathscr{A})$ является симплициальным комплексом. По построению $K(\mathscr{C} \mathscr{A})$ зависит лишь от $|\mathscr{C} \mathscr{A}|($ т.е. от $U(\mathscr{C} \mathscr{A}))$ и $U(K(\mathscr{C} \mathscr{A}))=U(\mathscr{C} \mathscr{A})$, откуда и вытекает утверждение.

Если координатная конфигурация $\mathscr{A}$ содержит некоторую гиперплоскость, скажем, $\left\{z_{i}=0\right\}$, то ее дополнение $U(\mathscr{A})$ раскладьвается как $U\left(\mathscr{A}_{0}\right) \times \mathbb{C}^{*}$, где $\mathscr{A}_{0}-$ координатная конфигурация в гиперплоскости $\left\{z_{i}=0\right\}$ и $\mathbb{C}^{*}=\mathbb{C} \backslash\{0\}$. Таким образом, для любой координатной конфигурации $\mathscr{A}$ дополнение $U(\mathscr{A})$ представляется в виде

$$
U(\mathscr{A})=U\left(\mathscr{A}^{\prime}\right) \times\left(\mathbb{C}^{*}\right)^{k},
$$

где $\mathscr{A}^{\prime}$ - конфигурация координатных подпространств в $\mathbb{C}^{m-k}$, которая не содержит гиперплоскостей. С другой стороны, (74) показывает, что $\mathscr{C} \mathscr{A}$ содержит гиперплоскость $\left\{z_{i}=0\right\}$ тогда и только тогда, когда $\{i\}$ не является вершиной комплекса $K(\mathscr{C} \mathscr{A})$. Отсюда вытекает, что $U(K)$ является дополнением координатной конфигурации, не содержашей гиперплоскостей, тогда и только тогда, когда множество вершин комплекса $K$ есть все $[m]$. Принимая во внимание эти замечания, мы ограничимся рассмотрением координатных конфигураций без гиперплоскостей и симплициальных комплексов на множестве вершин $[m]$. 
ЗАмЕчАнИЕ. Используя конструкцию 3.5.1, мы имеем $U(K)=K \bullet\left(\mathbb{C}, \mathbb{C}^{*}\right)$.

ПримеР 5.2.3. 1. Если $K=\Delta^{m-1}((m-1)$-симплекс $)$, то $U(K)=\mathbb{C}^{m}$.

2. Если $K=\partial \Delta^{m-1}$ (граница симплекса), то $U(K)=\mathbb{C}^{m} \backslash\{0\}$.

3. Если $K$ представляет собой несвязное объединение $m$ вершин, то $U(K)$ получается удалением из $\mathbb{C}^{m}$ всех координатных подпространств коразмерности два (т.е. вида $\left.z_{i}=z_{j}=0, i, j=1, \ldots, m\right)$.

Действие алгебраического тора $\left(\mathbb{C}^{*}\right)^{m}$ на $\mathbb{C}^{m}$ индуцирует действие на $U(K)$. В частности, на $U(K)$ определено стандартное действие тора $T^{m}$. Фактор-пространство $U(K) / T^{m}$ можно отождествить с пересечением $U(K) \cap \mathbb{R}_{+}^{m}$, где $\mathbb{R}_{+}^{m}$ рассматривается как подмножество в $\mathbb{C}^{m}$.

ЛЕмма 5.2.4. $\operatorname{cc}(K) \subset U(K) \cap \mathbb{R}_{+}^{m} u \mathscr{Z}_{K} \subset U(K)$ (см. конструкиию 1.5.9 и (39)).

ДокаЗАтельство. Рассмотрим $y=\left(y_{1}, \ldots, y_{m}\right) \in \operatorname{cc}(K)$. Пусть $I=\left\{i_{1}, \ldots, i_{k}\right\}$ обозначает максимальное подмножество в $[m]$ такое, что $y \in L_{I} \cap \mathbb{R}_{+}^{n}$ (т.е. $y_{i_{1}}=\cdots=$ $\left.y_{i_{k}}=0\right)$. Тогда из определения сc $(K)$ (см. (22)) вытекает, что $I$ является симплексом в $K$. Следовательно, $L_{I} \notin \mathscr{C} \mathscr{A}(K)$ и $y \in U(K)$. Таким образом, первое утверждение доказано. Так как сс $(K)$ является пространством орбит для действия тора $T^{m}$ на $\mathscr{Z}_{K}$, второе утверждение следует из первого.

ТЕОРема 5.2.5. Имеет место әквивариантная деформационная ретракиия $U(K) \rightarrow \mathscr{Z}_{K}$

ДоКАЗАТЕЛЬСТво. Вначале мы построим деформационную ретракцию $r: U(K) \cap$ $\mathbb{R}_{+}^{m} \rightarrow \operatorname{cc}(K)$. Это делается по индукции. Мы начнем с граничного комплекса $(m-1)$-симплекса и будем удалять симплексы положительных размерностей, пока не получим $K$. На каждом шаге мы будем строить деформационную ретракцию, и композиция всех этих ретракций даст требуемую ретракцию $r$.

Если $K=\partial \Delta^{m-1}$ - граничный комплекс $(m-1)$-симплекса, то $U(K) \cap \mathbb{R}_{+}^{m}=$ $\mathbb{R}_{+}^{m} \backslash\{0\}$. В этом случае ретракция $r$ показана на рис. 11.

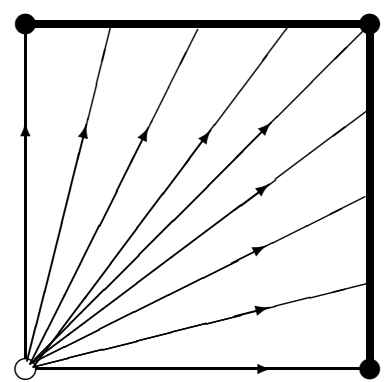

Рис. 11. Ретракция $r: U(K) \cap \mathbb{R}_{+}^{m} \rightarrow \operatorname{cc}(K)$ для $K=\partial \Delta^{m-1}$

Пусть теперь $K$ получается удалением одного $(k-1)$-мерного симплекса $J=\left\{j_{1}\right.$, $\left.\ldots, j_{k}\right\}$ из симплишиального комплекса $K^{\prime}$, т.е. $K \cup J=K^{\prime}$. По предположению индукции имеется деформационная ретракция $r^{\prime}: U\left(K^{\prime}\right) \cap \mathbb{R}_{+}^{m} \rightarrow \operatorname{cc}\left(K^{\prime}\right)$. Пусть $a \in \mathbb{R}_{+}^{m}-$ точка с координатами $y_{j_{1}}=\cdots=y_{j_{k}}=0, y_{i}=1$ при $i \notin J$. Так как $J-$ не симплекс 
комплекса $K$, мы имеем $a \notin U(K) \cap \mathbb{R}_{+}^{m}$. В то же время, $a \in C_{J}$ (см. (19)). Таким образом, мы можем применить ретракцию, изображенную на рис. 11 , на грани $C_{J} \subset I^{m}$ с центром в точке $a$. Обозначим эту ретракцию через $r_{J}$. Тогда $r=r_{J} \circ r^{\prime}-$ требуемая деформационная ретракция.

Деформационная ретракция $r: U(K) \cap \mathbb{R}_{+}^{m} \rightarrow \operatorname{cc}(K)$ накрывается эквивариантной деформационной ретракцией $U(K) \rightarrow \mathscr{Z}_{K}$.

В случае, когда $K=K_{P}$ есть многогранная симплициальная сфера, соответствуюшая простому многограннику $P^{n}$, деформационная ретракция $U\left(K_{P}\right) \rightarrow \mathscr{Z}_{P}$ из теоремы 5.2.5 может быть реализована как проекция на пространство орбит для действия стягиваемой групшы. Введем обозначение $U\left(P^{n}\right):=U\left(K_{P}\right)$. Положим

$$
\mathbb{R}_{>}^{m}=\left\{\left(y_{1}, \ldots, y_{m}\right) \in \mathbb{R}^{m}: y_{i}>0, i=1, \ldots, m\right\} \subset \mathbb{R}_{+}^{m} .
$$

Тогда $\mathbb{R}_{>}^{m}$ является группой по умножению, которая действует покоординатным умножением на $\mathbb{R}^{m}, \mathbb{C}^{m}$ и $U\left(P^{n}\right)$. Имеет место изоморфизм $\exp : \mathbb{R}^{m} \rightarrow \mathbb{R}_{>}^{m}$ между аддитивной и мультипликативной группой, которьй переводит $\left(y_{1}, \ldots, y_{m}\right) \in \mathbb{R}^{m}$ в $\left(e^{y_{1}}, \ldots, e^{y_{m}}\right) \in \mathbb{R}_{>}^{m}$.

Рассмотрим матрищу $W$ размера $m \times(m-n)$, построенную в конструкции 1.1 .4 по простому многограннику (1).

ПРЕДЛОЖЕНИЕ 5.2.6. ДЛя любой вериинъ $v=F_{i_{1}} \cap \cdots \cap F_{i_{n}}$ многогранника $P^{n}$ максимальный минор $W_{\widehat{i}_{1} \ldots \widehat{i}_{n}}$ матрицы $W$, получаемый удалением $n$ строк $i_{1}, \ldots, i_{n}$, невырожсден: $\operatorname{det} W_{\widehat{i}_{1} \ldots \widehat{i}_{n}} \neq 0$.

ДокаЗАТЕльство. Если $\operatorname{det} W_{\widehat{i}_{1}} \ldots \widehat{i}_{n}=0$, то векторы $\boldsymbol{l}_{i_{1}}, \ldots, \boldsymbol{l}_{i_{n}}$ (см. (1)) линейно зависимы, что невозможно.

Матрица $W$ определяет подгруппу

$$
R_{W}=\left\{\left(e^{w_{11} \tau_{1}+\cdots+w_{1, m-n} \tau_{m-n}}, \ldots, e^{w_{m 1} \tau_{1}+\cdots+w_{m, m-n} \tau_{m-n}}\right)\right\} \subset \mathbb{R}_{>}^{m}
$$

где набор параметров $\left(\tau_{1}, \ldots, \tau_{m-n}\right)$ пробегает пространство $\mathbb{R}^{m-n}$. Очевидно, $R_{W} \cong \mathbb{R}_{>}^{m-n}$.

Tеорема 5.2 .7 [22; теорема 2.3]. Подгруппа $R_{W} \subset \mathbb{R}_{>}^{m}$ действует на $U\left(P^{n}\right) \subset$ $\mathbb{C}^{m}$ свободно. Композичия $\mathscr{Z}_{P} \hookrightarrow U\left(P^{n}\right) \rightarrow U\left(P^{n}\right) / R_{W}$ вложсения $i_{e}$ (лемма 3.1.6) и проекиии на пространство орбит является әквивариантньми (относительно действия $T^{m}$ ) диффеоморфизмом.

ДокАЗАТЕльство. Точка в $\mathbb{C}^{m}$ имеет нетривиальную стационарную подгрупшу относительно действия $\mathbb{R}_{>}^{m}$ на $\mathbb{C}^{m}$ тогда и только тогда, когда хотя бы одна из ее координат обращается в нуль. Из (73) вытекает, что если точка $x \in U\left(P^{n}\right)$ имеет некоторые нулевые координаты, то соответствующие этим координатам гиперграни $P^{n}$ имеют хотя бы одну обшую вершину $v \in P^{n}$. Пусть $v=F_{i_{1}} \cap \cdots \cap F_{i_{n}}$. Стационарная подгруппа точки $x$ относительно действия подгруппы $R_{W}$ нетривиальна, только если некоторая линейная комбинация столбцов матрицы $W$ лежит в координатном пространстве, порожденном $\boldsymbol{e}_{i_{1}}, \ldots, \boldsymbol{e}_{i_{n}}$. Но это означает, что $\operatorname{det} W_{\widehat{i}_{1}} \ldots \widehat{i}_{n}=0$, что противоречит предложению 5.2.6. Итак, $R_{W}$ действует на $U\left(P^{n}\right)$ свободно. 
Для доказательства второй части теоремы достаточно показать, что каждая орбита действия $R_{W}$ на $U\left(P^{n}\right) \subset \mathbb{C}^{m}$ пересекает образ $i_{e}\left(\mathscr{Z}_{P}\right)$ в единственной точке. Так как вложение $i_{e}$ эквивариантно относительно действия $T^{m}$, последнее утверждение эквивалентно тому, что каждая орбита действия $R_{W}$ на $U\left(P^{n}\right) \cap \mathbb{R}_{+}^{m}$ пересекает образ $i_{P}\left(P^{n}\right)$ (см. теорему 1.5.6) в единственной точке. Пусть $y \in i_{P}\left(P^{n}\right) \subset \mathbb{R}^{m}$. Тогда $y=\left(y_{1}, \ldots, y_{m}\right)$ лежит на некоторой $n$-грани $i_{P}\left(C_{v}^{n}\right)$ куба $I^{m} \subset \mathbb{R}^{m}$, см. (20). Мы должны доказать, что $(m-n)$-мерное подпространство, порожденное векторами $\left(w_{11} y_{1}, \ldots, w_{m 1} y_{m}\right)^{t}, \ldots,\left(w_{1, m-n} y_{1}, \ldots, w_{m, m-n} y_{m}\right)^{t}$, находится в общем положении с $n$-гранњю $i_{P}\left(C_{v}^{n}\right)$ куба $I^{m}$. Но это вытекает непосредственно из (20) и предложения 5.2.6.

Предположим теперь, что $P^{n}$ является решеточным простьм многогранником, и пусть $M_{P}$ - соответствуюшее торическое многообразие (конструкция 2.1.3). Наряду с вешественной подгруппой $R_{W} \subset \mathbb{R}_{>}^{m}(75)$ определим

$$
C_{W}=\left\{\left(e^{w_{11} \phi_{1}+\cdots+w_{1, m-n} \phi_{m-n}}, \ldots, e^{w_{m 1} \phi_{1}+\cdots+w_{m, m-n} \phi_{m-n}}\right)\right\} \subset\left(\mathbb{C}^{*}\right)^{m},
$$

где набор параметров $\left(\phi_{1}, \ldots, \phi_{m-n}\right)$ пробегает пространство $\mathbb{C}^{m-n}$. Очевидно, $C_{W} \cong\left(\mathbb{C}^{*}\right)^{m-n}$. В работах [7], [11], [31] показано, что $C_{W}$ действует свободно на $U\left(P^{n}\right)$ и торическое многообразие $M_{P}$ может быть отождествлено с пространством орбит (или геометрическим фактором) $U\left(P^{n}\right) / C_{W}$. Таким образом, мы имеем коммутативную диаграмму

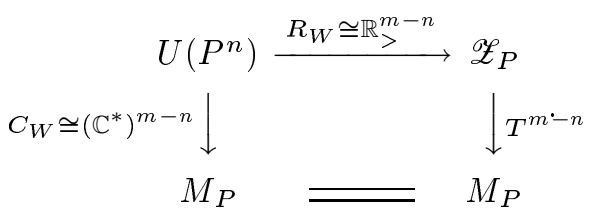

ЗАмЕчАНИЕ. Можно показать [31; теорема 2.1], что любое торическое многообразие $M_{\Sigma}$, построенное по вееру $\Sigma \subset \mathbb{R}^{n}$ с $m$ одномерньпи конусами, отождествляется с универсальным категорным фактор-пространством $U\left(\mathscr{C} \mathscr{A}_{\Sigma}\right) / G$, где $U\left(\mathscr{C}_{\Sigma}\right)$ дополнение некоторой координатной конфигурации, определяемой веером $\Sigma$, a $G \cong$ $\left(\mathbb{C}^{*}\right)^{m-n}$. Категорньй фактор является геометрическим фактором тогда и только тогда, когда веер $\Sigma$ симплициален. В этом случае $U\left(\mathscr{C}_{\Sigma}\right)=U\left(K_{\Sigma}\right)$.

$\mathrm{C}$ другой стороны, если проективное торическое многообразие $M_{P}$ неособо, то $M_{P}$ является симплектическим многообразием размерности $2 n$ и действие тора $T^{n}$ на нем гамильтоново [7]. В этом случае диаграмма (76) описьвает $M_{P}$ как результат так назьваемой симплектической редукиии. А именно, пусть $H_{W} \cong T^{m-n}$ - максимальная компактная подгруппа в $C_{W}$ и $\mu: \mathbb{C}^{m} \rightarrow \mathbb{R}^{m-n}-$ отображсение моментов для гамильтонова действия групшы $H_{W}$ на $\mathbb{C}^{m}$. Тогда для любого регулярного значения $a \in \mathbb{R}^{m-n}$ отображения $\mu$ имеет место диффеоморфизм

$$
\mu^{-1}(a) / H_{W} \longrightarrow U\left(P^{n}\right) / C_{W}=M_{P}
$$

(подробности см. в [7]). Тогда $\mu^{-1}(a)$ есть в точности наше многообразие $\mathscr{Z}_{P}$. Таким образом, мы получаем еще одну интерпретацию многообразия $\mathscr{Z}_{P}$ как многообразия уровня отображения моментов (в случае, когда $P^{n}$ допускает геометрическую реализацию, дающую неособое торическое многообразие). 
ПримеР 5.2.8. Пусть $P^{n}=\Delta^{n}$ (n-симплекс). Тогда $m=n+1, U\left(P^{n}\right)=\mathbb{C}^{n+1} \backslash\{0\}$, а $R_{W} \cong \mathbb{R}_{>}, C_{W} \cong \mathbb{C}^{*}$ и $H_{W} \cong S^{1}$ - диагональные подгруппы в $\mathbb{R}_{>}^{n+1},\left(\mathbb{C}^{*}\right)^{n+1}$ и $T^{m+1}$ соответственно (см. пример 1.1.5). Итак, $\mathscr{Z}_{P} \cong S^{2 n+1}=\left(\mathbb{C}^{n+1} \backslash\{0\}\right) / \mathbb{R}_{>}$ и $M_{P}=\left(\mathbb{C}^{n+1} \backslash\{0\}\right) / \mathbb{C}^{*}=\mathbb{C} P^{n}$. Отображение моментов $\mu: \mathbb{C}^{m} \rightarrow \mathbb{R}$ переводит $\left(z_{1}, \ldots, z_{m}\right) \in \mathbb{C}^{m}$ В $\frac{1}{2}\left(\left|z_{1}\right|^{2}+\cdots+\left|z_{m}\right|^{2}\right)$, и для $a \neq 0$ мы имеем $\mu^{-1}(a) \cong S^{2 n+1} \cong \mathscr{Z}_{K}$.

Предыдущие обсуждения иллюстрируют важность вычисления когомологий дополнений конфигураций координатных подпространств.

Теорема 5.2.9 (Бухштабер, Панов). Имеет место следующий изоморфизм градуированных алгебр:

$$
H^{*}(U(K)) \cong \operatorname{Tor}_{\mathbf{k}\left[v_{1}, \ldots, v_{m}\right]}(\mathbf{k}(K), \mathbf{k})=H\left[\Lambda\left[u_{1}, \ldots, u_{m}\right] \otimes \mathbf{k}(K), d\right] .
$$

ДокАЗАТЕЛЬСТво. Это вытекает из теорем 5.2.5, 4.2.1 и 4.2.2.

Теорема 5.2.9 дает весьма эффективньй способ вычисления алгебры когомологий дополнения произвольной конфигурации комплексных координатных подпространств. Модели этой алгебры, использованные де Кончини и Прочези [34] и Юзвинским [88], также можно интерпретировать как приложение резольвенты Кошуля. Однако этими авторами не обсуждались взаимоотношения с кольцом Стенли-Райснера в случае координатных конфигураций.

ПроБлема 5.2.10. Описать алгебру когомологий с коэффициентами в $\mathbb{Z}$ дополнения конфигурации координатных подпространств и связать ее с соответствующей Tor-алгеброй $\operatorname{Tor}_{\mathbb{Z}\left[v_{1}, \ldots, v_{m}\right]}(\mathbb{Z}(K), \mathbb{Z})$.

ПримеР 5.2.11. Пусть $K$ - несвязное объединение $m$ вершин. Тогда $U(K)$ получается удалением из $\mathbb{C}^{m}$ всех координатных подпространств коразмерности два, т.е. вида $z_{i}=z_{j}=0, i, j=1, \ldots, m$ (см. пример 5.2.3). Кольцо граней есть $\mathbf{k}(K)=$ $\mathbf{k}\left[v_{1}, \ldots, v_{m}\right] / \mathscr{I}_{K}$, где идеал $\mathscr{I}_{K}$ порожден мономами $v_{i} v_{j}, i \neq j$. Простое вычисление с использованием теоремы 5.2.9 показывает, что подпространство коциклов в $\mathbf{k}(K) \otimes \Lambda\left[u_{1}, \ldots, u_{m}\right]$ имеет базис, состояший из мономов $v_{i_{1}} u_{i_{2}} u_{i_{3}} \cdots u_{i_{k}}$ с $k \geqslant 2$, $i_{p} \neq i_{q}$ при $p \neq q$. Так как $\operatorname{deg}\left(v_{i_{1}} u_{i_{2}} u_{i_{3}} \cdots u_{i_{k}}\right)=k+1$, пространство $(k+1)$-мерных коциклов имеет размерность $m\left(\begin{array}{c}m-1 \\ k-1\end{array}\right)$. Пространство $(k+1)$-мерных когранищ имеет размерность $\left(\begin{array}{c}m \\ k\end{array}\right)$ (оно порождается кограницами вида $d\left(u_{i_{1}} \cdots u_{i_{k}}\right)$ ). Следовательно,

$$
\begin{gathered}
\operatorname{dim} H^{0}(U(K))=1, \quad H^{1}(U(K))=H^{2}(U(K))=0, \\
\operatorname{dim} H^{k+1}(U(K))=m\left(\begin{array}{c}
m-1 \\
k-1
\end{array}\right)-\left(\begin{array}{c}
m \\
k
\end{array}\right)=(k-1)\left(\begin{array}{c}
m \\
k
\end{array}\right), \quad 2 \leqslant k \leqslant m,
\end{gathered}
$$

а умножение в когомологиях тривиально.

В частности, при $m=3$ мы имеем 6 трехмерных классов когомологий $\left[v_{i} u_{j}\right], i \neq j$, с 3 соотношениями $\left[v_{i} u_{j}\right]=\left[v_{j} u_{i}\right]$ и 3 четырехмерных класса когомологий $\left[v_{1} u_{2} u_{3}\right]$, $\left[v_{2} u_{1} u_{3}\right],\left[v_{3} u_{1} u_{2}\right]$ с одним соотношением

$$
\left[v_{1} u_{2} u_{3}\right]-\left[v_{2} u_{1} u_{3}\right]+\left[v_{3} u_{1} u_{2}\right]=0 .
$$

Следовательно, $\operatorname{dim} H^{3}(U(K))=3, \operatorname{dim} H^{4}(U(K))=2$, а умножение тривиально. 
ПримеР 5.2.12. Пусть $K$ - гранища $m$-угольника на плоскости с $m \geqslant 3$. Тогда

$$
U(K)=\mathbb{C}^{m} \backslash \bigcup_{i-j \neq 0,1 \bmod m}\left\{z_{i}=z_{j}=0\right\} .
$$

Из теоремы 5.2.9 вытекает, что кольцо когомологий $H^{*}(U(K) ; \mathbf{k})$ изоморфино кольцу, описанному в примере 4.3 .5 .

Как показано в [41], в случае конфигураций вещественны $x$ координатных подпространств имеет место лишь аддитивный аналог нашей теоремы 5.2.9. А именно, рассмотрим теперь кольцо многочленов $\mathbf{k}\left[x_{1}, \ldots, x_{m}\right]$, в котором $\operatorname{deg} x_{i}=1, i=1, \ldots, m$, и соответственно сменим градуировки в кольце граней $\mathbf{k}(K)$. Тогда числа Бетти вещественной координатной конфигурации $U_{\mathbb{R}}(K)$ вычисляются при помощи следуюшего результата.

ТеОРема 5.2.13 [41; теорема 3.1]. Имеют место изоморфизмы

$$
H^{p}\left(U_{\mathbb{R}}(K)\right) \cong \sum_{-i+j=p} \operatorname{Tor}_{\mathbf{k}\left[x_{1}, \ldots, x_{m}\right]}^{-i, j}(\mathbf{k}(K), \mathbf{k})=H^{-i, j}\left[\Lambda\left[u_{1}, \ldots, u_{m}\right] \otimes \mathbf{k}(K), d\right],
$$

əде bideg $u_{i}=(-1,1), \operatorname{bideg} v_{i}=(0,1), d u_{i}=x_{i}, d x_{i}=0$.

Как было отмечено в [41], мультипликативньй изоморфизм, аналогичньй теореме 5.2.9, для вещественных конфигураций не имеет места, т.е. алгебры $H^{*}\left(U_{\mathbb{R}}(K)\right)$ и $\operatorname{Tor}_{\mathbf{k}\left[x_{1}, \ldots, x_{m}\right]}(\mathbf{k}(K), \mathbf{k})$, вообще говоря, не изоморфны. В [41] также содержится формулировка первого мультипликативного изоморфизма из нашей теоремы 5.2.9 для комплексных координатных конфигураций (см. [41; теорема 3.6]) со ссылкой на неопубликованную работу Бабсона и Чана.

До сих пор мы использовали описание координатных подпространств при помощи уравнений (см. (72)). С другой стороны, их можно задавать в виде линейных оболочек соответствующих подмножеств множества базисных векторов $\left\{\boldsymbol{e}_{1}, \ldots, \boldsymbol{e}_{m}\right\}$. Это приводит к двойственному подходу в описании конфигураций координатных подпространств, который соответствует переходу от симплициального комплекса $K$ к ассоциированному симплициальному комплексу $\widehat{K}$ (пример 1.2.4). Этот подход использован в [35]. Там было показано, что слагаемые в формуле Горески-Макферсона (теорема 5.1.2) в случае координатных конфигураций являются группами гомологий линков симплексов из $\widehat{K}$. Это позволило интерпретировать операцию умножения классов когомологий дополнения конфигурации координатных подпространств (вешественных или комплексных) в терминах комбинаторики линков симплексов комплекса $\widehat{K}$ (см. [35; теорема 1.1]).

Обратим внимание, что наши теоремы 4.2.2 и 5.2.5 позволяют установить эквивалентность результата Горески-Макферсона (теорема 5.1.2) в случае конфигураций координатных подпространств и теоремы Хохстера (теорема 1.4.5).

§ 5.3. Конфигурации диагональных подпространств и когомологии пространства петель $\Omega \mathscr{Z}_{K}$. В настояшем параграфе мы устанавливаем взаимосвязи между результатами работы [71] о когомологиях дополнений вещественных диагональных координатных подпространств и когомологиями пространств петель $\Omega B_{T} \mathscr{Z}_{K}$ и $\Omega \mathscr{Z}_{K}$. 
Для каждого подмножества $I=\left\{i_{1}, \ldots, i_{k}\right\} \subset[m]$ определим диагональное подпространство $D_{I}$ в $\mathbb{R}^{m}$ как

$$
D_{I}=\left\{\left(y_{1}, \ldots, y_{m}\right) \in \mathbb{R}^{m}: y_{i_{1}}=\cdots=y_{i_{k}}\right\} .
$$

Аналогично определяются диагональные подпространства в $\mathbb{C}^{m}$. Конфигурация $\mathscr{A}=\left\{L_{1}, \ldots, L_{r}\right\}$ (вещественная или комплексная) назьвается диагональной, если все плоскости $L_{i}, i=1, \ldots, r$, являются диагональными подпространствами. Классическим примером комплексной диагональной конфигурации является конфигурация всевозможных диагональных гиперплоскостей $\left\{z_{i}=z_{j}\right\}$ в $\mathbb{C}^{m}$, дополнение которой представляет собой классифицируюшее пространство для группы кос $B_{m}$, см. [4].

Конструкция 5.3.1. Для любого симплициального комплекса $K$ на множестве вершин $[m]$ введем вешественную диагональную конфигурацию $\mathscr{D} \mathscr{A}(K)$ как множество подпространств $D_{I}$, где $I$ не является симплексом в $K$ :

$$
\mathscr{D} \mathscr{A}(K)=\left\{D_{I}: I \notin K\right\} .
$$

Обозначим дополнение конфигурации $\mathscr{D} \mathscr{A}(K)$ через $M(K)$.

Аналогично тому, как это делалось в $§ 5.2$ для координатных конфигураций, доказьвается следуюшее утверждение.

ПРеДЛОЖЕнИЕ 5.3.2. Сопоставление $K \mapsto M(K)$ определяет сохраняющее порядок взаимно однозначное соответствие между симплициальными комплексами на множестве вериин $[\mathrm{m}]$ и дополнениями конфигураций диагональных подпространств в $\mathbb{R}^{m}$.

Здесь мы, как и ранее, предполагаем, что $\mathbf{k}$ - поле. Мультиградуированная (или $\mathbb{N}^{m}$-градуированная) структура в кольце $\mathbf{k}\left[v_{1}, \ldots, v_{m}\right]$ (конструкция 1.4.7), определяет $\mathbb{N}^{m}$-градуировку в кольце Стенли-Райснера $\mathbf{k}(K)$. При этом моном $v_{1}^{i_{1}} \cdots v_{m}^{i_{m}}$ приобретает мультистепень $\left(2 i_{1}, \ldots, 2 i_{m}\right)$. Рассмотрим модули $\operatorname{Tor}_{\mathbf{k}(K)}(\mathbf{k}, \mathbf{k}) ;$ их можно вычислять, например, при помоши минимальной свободной резольвенты (пример 1.4.2) поля $\mathbf{k}$, рассматриваемого как $\mathbf{k}(K)$-модуль. Минимальная резольвента также несет естественную $\mathbb{N}^{m}$-градуировку, и мы будем обозначать подгруппу элементов мультиградуировки $\left(2 i_{1}, \ldots, 2 i_{m}\right)$ в $\operatorname{Tor}_{\mathbf{k}(K)}(\mathbf{k}, \mathbf{k})$ через $\operatorname{Tor}_{\mathbf{k}(K)}(\mathbf{k}, \mathbf{k})_{\left(2 i_{1}, \ldots, 2 i_{m}\right)}$.

Теорема 5.3.3 [71; теорема 1.3]. Для групп когомологий дополнения $M(K)$ вещественной конфигурации диагональных подпространств имеет место изомор$\phi и з м$

$$
H^{i}(M(K) ; \mathbf{k}) \cong \operatorname{Tor}_{\mathbf{k}(K)}^{-(m-i)}(\mathbf{k}, \mathbf{k})_{(2, \ldots, 2)} .
$$

ЗАмечАнИЕ. Вместо симплипиальных комплексов $K$ на множестве вершин $[m]$ в [71] рассматривались мономиальные идеалы $\mathscr{I} \subset \mathbf{k}\left[v_{1}, \ldots, v_{m}\right]$, имеюшие базис из мономов без квадратов. Эквивалентность этих двух подходов вытекает из предложения 1.3.2. 
ТЕОРема 5.3.4. Имеет место аддитивный изоморфизм

$$
H^{*}\left(\Omega B_{T} \mathscr{Z}_{K} ; \mathbf{k}\right) \cong \operatorname{Tor}_{\mathbf{k}(K)}(\mathbf{k}, \mathbf{k}) .
$$

ДокАЗАТЕЛьство. Рассмотрим спектральную последовательность ЭйленбергаМура расслоения в смысле Серра $P \rightarrow S R(K)$ со слоем $\Omega S R(K)$, где $S R(K)$ - пространство Стенли-Райснера (определение 3.4.2), а $P$ - пространство путей над $S R(K)$. В силу следствия 4.1.4 мы имеем

$$
E_{2}=\operatorname{Tor}_{H^{*}(S R(K))}\left(H^{*}(P), \mathbf{k}\right) \cong \operatorname{Tor}_{\mathbf{k}(K)}(\mathbf{k}, \mathbf{k})
$$

и спектральная последовательность сходится к

$$
\operatorname{Tor}_{C^{*}(S R(K))}\left(C^{*}(P), \mathbf{k}\right) \cong H^{*}(\Omega S R(K)) .
$$

Так как $P$ стягиваемо, мы имеем коцепную эквивалентность $C^{*}(P) \simeq \mathbf{k}$. Кроме того, $C^{*}(S R(K)) \cong \mathbf{k}(K)$. Следовательно,

$$
\operatorname{Tor}_{C^{*}(S R(K))}\left(C^{*}(P), \mathbf{k}\right) \cong \operatorname{Tor}_{\mathbf{k}(K)}(\mathbf{k}, \mathbf{k}),
$$

что вместе с (77) показывает, что спектральная последовательность вырождается в члене $E_{2}$, т.е.

$$
H^{*}(\Omega S R(K)) \cong \operatorname{Tor}_{\mathbf{k}(K)}(\mathbf{k}, \mathbf{k}) .
$$

Наконец, теорема 3.4.4 показьвает, что

$$
H^{*}(\Omega S R(K)) \cong H^{*}\left(\Omega B_{T} \mathscr{Z}_{K}\right)
$$

что завершает доказательство.

ПРЕДЛОЖЕНИЕ 5.3.5. Имеет место изоморфизм алгебр

$$
H^{*}\left(\Omega B_{T} \mathscr{Z}_{K}\right) \cong H^{*}\left(\Omega \mathscr{Z}_{K}\right) \otimes \Lambda\left[u_{1}, \ldots, u_{m}\right] .
$$

ДокаЗАтельство. Рассмотрим расслоение $B_{T} \mathscr{Z}_{K} \rightarrow B T^{m}$ со слоем $\mathscr{Z}_{K}$. Нетрудно доказать, что соответствующее расслоение петель $\Omega B_{T} \mathscr{Z}_{K} \rightarrow T^{m}$ со слоем $\Omega \mathscr{Z}_{K}$ тривиально (заметим, что $\Omega B T^{m} \cong T^{m}$ ). Для доказательства утверждения остается заметить, что $H^{*}\left(T^{m}\right) \cong \Lambda\left[u_{1}, \ldots, u_{m}\right]$.

Теоремы 5.2.5 и 5.2.9 позволяют использовать теорию момент-угол комплексов для выгисления кольца когомологий дополнения координатной конфигурации. Аналогично, теоремы 5.3.3, 5.3.4 и предложение 5.3.5 позволяют связать когомологии дополнений конфигураций диагональных подпространств и когомологии пространства петель над момент-угол комплексом $\mathscr{Z}_{K}$. Однако в этом случае ситуация значительно сложнее, чем в случае конфигураций координатных подпространств. В частности, мы не имеем аналога мультипликативного изоморфизма из теоремы 5.2.9. Поэтому данный заключительньй параграф̆ мы рассматриваем лишш как первьй шаг в изучении дополнений диагональных конфигураций при помощи теории момент-угол комплексов. 


\section{СПИСОК ЛИТЕРАТУРЫ}

[1] Adams J.F. On the cobar construction // Proc. Nat. Acad. Sci. USA. 1956. V. 42. P. 409-412.

[2] Adams J. F. On the non-existence of elements of Hopf invariant one // Ann. of Math. (2). 1960. V. 72. № 1. P. 20-104.

[3] Akbulut S., McCarthy J. D. Casson's Invariant for Oriented Homology 3-spheres. Princeton, NJ: Princeton Univ. Press, 1990.

[4] Арнольд В.И. Кольцо когомологий группы крашенных кос // Матем. заметки. 1969. T. 5. C. $227-231$.

[5] Atiyah M.F., Bott R. A Lefschetz fixed point formula for elliptic complexes. I // Ann. of Math. (2). 1967. V. 86. № 2. P. 374-407.

[6] Atiyah M.F., Hirzebruch F. Spin-manifolds and group actions // Essays in Topology and Related Topics. Berlin: Springer-Verlag, 1970. P. 18-28.

[7] Audin M. The Topology of Torus Actions on Symplectic Manifolds. Boston: Birkhäuser, 1991.

[8] Bahri A., Bendersky M. The KO-theory of toric manifolds // Trans. Amer. Math. Soc. 2000. V. 352. № 3. P. 1191-1202; http://xxx.lanl.gov/abs/math.AT/9904087.

[9] Barnette D. The triangulations of the 3 -sphere with up to 8 vertices // J. Combinatorial Theory. Ser. A. 1973. V. 14. P. 37-42.

[10] Barnette D. A proof of the lower bound conjecture for convex polytopes // Pacific J. Math. 1973. V. 46. P. 349-354.

[11] Batyrev V. V. Quantum cohomology rings of toric manifolds // Journées de Géométrie Algébrique d'Orsay (Juillet, 1992). Paris: Sociéte Mathématique de France, 1993. P. 9-34 (Astérisque. V. 218.); http://xxx.lanl.gov/abs/alg-geom/9310004.

[12] Bayer M., Billera L. Generalized Dehn-Sommerville relations for polytopes, spheres and Eulerian partially ordered sets // Invent. Math. 1985. V. 79. P. 143-157.

[13] Billera L., Lee C. Sufficiency of McMullen's conditions for $f$-vectors of simplicial polytopes // Bull. Amer. Math. Soc. 1980. V. 2. P. 181-185.

[14] Björner A. Subspace arrangements // Proc. of the First European Congress of Mathematics (Paris, 1992). V. 1 / ed. A. Joseph et al. Birkhäuser: Basel, 1994. P. 321-370.

[15] Björner A., Lutz F. Simplicial manifolds, bistellar flips and 16-vertex triangulation of the Poincaré homology 3-sphere // Preprint, 1999; http://www.math.kth.se/ ^ bjorner/ papers.html.

[16] Boyer C., Galicki K., Mann B., Rees E. Compact 3-Sasakian 7-manifolds with arbitrary second Betti number // Invent. Math. 1998. V. 131. P. 321-344.

[17] Brieskorn E. Sur les groupes de tresses (d'après V.I. Arnol'd) // Séminaire Bourbaki 1971/72. Berlin: Springer-Verlag, 1973. P. 21-44. (Lecture Notes in Math. V. 317.)

[18] Бренстед А. Введение в теорию выпуклых многогранников. М.: Мир, 1988.

[19] Бухштабер В. М., Панов Т. Е. Алгебраическая топология многообразий, определяемых простыми многогранниками // УМН. 1998. Т. 53. № 3. С. 195-196.

[20] Бухштабер В. М., Панов Т. Е.Действия тора и комбинаторика многогранников // Труды МИРАН. 1999. Т. 225. С. 96-131.

[21] Бухштабер В. М., Панов Т. Е. Действия тора, эквивариантные момент-угол-комплексы и конфигурации координатных подпространств // Зап. научн. семин. ПО МИРАН. 2000. T. 266. C. $29-50$.

[22] Buchstaber V.M., Panov T.E. Torus actions determined by simple polytopes // Contemp. Math. 2000. V. 258. P. 33-46.

[23] Бухштабер В. М., Панов Т. Е. Момент-угол комплексы и комбинаторика симплициалњных многообразий // УМН. 2000. Т. 55. № 3. С. 171-172.

[24] Buchstaber V.M., Ray N. Flag manifolds and the Landweber-Novikov algebra // Geometry \& Topology. 1998. V. 2. P. 79-101; http://www.maths.warwick.ac.uk/gt/.

[25] Бухштабер В. М., Рай Н. Торические многообразия и комплексные кобордизмы // УМH. 1998. Т. 53. №2 2. С. 139-140. 
[26] Buchstaber V. M., Ray N. Tangential structures on toric manifolds, and connected sums of polytopes // Preprint UMIST no. 2000/5, Manchester, 2000; Internat. Math. Res. Notices (to appear).

[27] Cannon J. W. Shrinking cell-like decompositions of manifolds. Codimension three // Ann. of Math. (2). 1979. V. 110. P. 83-112.

[28] Картан А., Эйленберг С. Гомологическая алгебра. М.: ИЛ, 1960.

[29] Charney R., Davis M. The Euler characteristic of a nonpositively curved, piecewise Euclidean manifold // Pacific J. Math. 1995. V. 171. P. 117-137.

[30] Chen B., Yan M. Singularity from Eulerian viewpoint // Труды МИРАН. 1998. Т. 221. C. $305-319$.

[31] Cox D. A. Recent developments in toric geometry // Proc. Sympos. Pure Math. 1997. V. 62. P. 389-436; http://xxx.lanl.gov/abs/alg-geom/9606016.

[32] Данилов В.И. Геометрия торических многообразий // УМН. 1978. Т. 33. № 2. C. $85-134$.

[33] Davis M., Januszkiewicz T. Convex polytopes, Coxeter orbifolds and torus actions // Duke Math. J. 1991. V. 62. № 2. P. 417-451.

[34] De Concini C., Procesi C. Wonderful models of subspace arrangements // Selecta Math. New Ser. 1995. V. 1. P. 459-494.

[35] De Longueville M. The ring structure on the cohomology of coordinate subspace arrangements // Math. Z. 2000. V. 233. №3. P. 553-577.

[36] Добринская Н.Э. Задача классификации квазиторических многообразий надзаданным простым полиэдром // Функц. анализ и прил. 2000. Т. 34. №4 (в печати).

[37] Долбилин Н.П., Штанько М.А., Штогрин М.И. Кубические подкомплексы в правильных решетках // Докл. АН СССР. 1986. Т. 291. № 2. С. 277-279.

[38] Edwards R. D. The topology of manifolds and cell-like maps // Proceedings of the International Congress of Mathematicians (Helsinki, 1978). Helsinki, 1980. P. 111-127.

[39] Eilenberg S., Moore J. C. Homology and fibrations. I // Comment. Math. Helv. 1966. V. 40. P. $199-236$.

[40] Fulton W. Introduction to Toric Varieties. Princeton, NJ: Princeton Univ. Press, 1993.

[41] Gasharov V., Peeva I., Welker V. Coordinate subspace arrangements and monomial ideals // Preprint, 1999; http://www.mathematik.uni-marburg.de/ welker/sections/ preprints.html.

[42] Горески М., Макферсон Р. Стратифицированная теория Морса. М.: Мир, 1991.

[43] Gromov M. Hyperbolic groups // Essays in Group Theory / ed. S. M. Gersten. New York: Springer-Verlag, 1987. P. 75-264. (Math. Sci. Res. Inst. Publ. V. 8.)

[44] Hattori A. Almost complex toric manifolds and complex line bundles // Banach Center Publ. V. 45, 1998. P. 95-114.

[45] Hirzebruch F., Berger T., Jung R. Manifolds and Modular Forms. Bonn: Max-PlancInstitut für Mathematik, 1994.

[46] Hochster M. Cohen-Macaulay rings, combinatorics, and simplicial complexes // Ring Theory II. Proc. Second Oklahoma Conference / ed. B.R. McDonald and R. Morris. New York: Dekker, 1977. P. 171-223.

[47] Изместьев И. В. Многообразия, определяемые простыми многогранниками, как конфигурационные пространства шарнирных механизмов // УМН. 2000. Т. 55. №1. С. 185-186.

[48] Изместьев И.В. 3-мерные многообразия, определяемые раскраской граней простого многогранника // Матем. заметки. 2001. Т. 66 (в печати).

[49] Jänich K. On the classification of $O(n)$-manifolds // Math. Ann. 1968. V. 176. P. 53-76.

[50] Хованский А.Г. Гиперплоские сечения многогранников, торические многообразия и дискретные группы в пространстве Лобачевского // Функц. анализ и прил. 1986. Т. 20. № 1. C. $50-61$.

[51] Kirby R. C., Siebenmann L. C. Foundational Essays on Topological Manifolds, Smoothings, and Triangulations. Princeton, NJ: Princeton Univ. Press, 1977. (Ann. of Math. Stud. V. 88.) 
[52] Klee V. A combinatorial analogue of Poincaré's duality theorem // Canad. J. Math. 1964. V. 16. P. $517-531$.

[53] Kleinschmidt P. A classification of toric varieties with few generators // Aequationes Math. 1988. V. 35. №2-3. P. 254-266.

[54] Landweber P.S. Homological properties of comodules over $M U_{*}(M U)$ and $B P_{*}(B P) / /$ Amer. J. Math. 1976. V. 98. P. 591-610.

[55] Leung N. C., Reiner V. The signature of a toric variety // Preprint, 1999; http://www . math. umn.edu/ ${ }^{\sim}$ reiner/Papers/papers.html.

[56] Маклейн С. Гомология. М.: Мир, 1966.

[57] Masuda M. Unitary toric manifolds, multi-fans and equivariant index // Tohoku Math. J. 1999. V. 51. P. 237-265.

[58] McMullen P. The numbers of faces of simplicial polytopes // Israel J. Math. 1971. V. 9. P. 559-570.

[59] McMullen P. On simple polytopes // Invent. Math. 1993. V. 113. P. 419-444.

[60] Международный математический конгресс в Эдинбурге 1958 г. (обзорные доклады). М.: Физматлит, 1962.

[61] Moise E. E. Geometric Topology in Dimensions 2 and 3. New York: Springer-Verlag, 1977.

[62] Novikov S. P. Topology I. Berlin: Springer-Verlag, 1996.

[63] Oda T. Convex Bodies and Algebraic Geometry. New York: Springer-Verlag, 1988.

[64] Orlik P., Raymond F. Actions of the torus on 4-manifolds // Trans. Amer. Math. Soc. 1970. V. 152. P. 531-559.

[65] Orlik P., Solomon L. Combinatorics and topology of complements of hyperplanes // Invent. Math. 1980. V. 56. P. 167-189.

[66] Orlik P., Terao H. Arrangements of Hyperplanes. New York: Springer-Verlag, 1992.

[67] Pachner U. Konstruktionsmethoden und das kombinatorische Homöomorphieproblem für Triangulationen kompakter semilinearer Mannigfaltigkeiten // Abh. Math. Sem. Univ. Hamburg. 1986. V. 57. P. 69-85.

[68] Pachner U. P.L. homeomorphic manifolds are equivalent by elementary shellings // European J. Combin. 1991. V. 12. P. 129-145.

[69] Панов Т. Е. Комбинаторные формулы для $\chi$-рода полиориентированного квазиторического многообразия // УМН. 1999. Т. 54. № 5. С. 169-170.

[70] Panov T. E. Hirzebruch genera of manifolds with torus actions // Preprint, 1999; http: // xxx.lanl.gov/abs/math.AT/9910083.

[71] Peeva I., Reiner V., Welker V. Cohomology of real diagonal subspace arrangements via resolutions // Compositio Math. 1999. V. 117. №1. P. 99-115.

[72] Понтрягин Л. С. Основы комбинаторной топологии. М.: Наука, 1986.

[73] Ranicki A. A. On the Hauptvermutung // The Hauptvermutung book, $K$-Monogr. Math. V. 1. Dordrecht: Kluwer Acad. Publ., 1996. P. 3-31.

[74] Reisner G. Cohen-Macaulay quotients of polynomial rings // Adv. Math. 1976. V. 21. P. 30-49.

[75] Рурк К., Сандерсон Б. Введение в кусочно-линейную топологию. М.: Мир, 1974.

[76] Штанько М. А., Штогрин М. И. О вложениях кубических многообразий и комплексов в кубическую решетку // УМН. 1992. Т. 47. № 1. С. 219-220.

[77] Smith L. Homological algebra and the Eilenberg-Moore spectral sequence // Trans. Amer. Math. Soc. 1967. V. 129. P. 58-93.

[78] Sommerville D. M. Y. The relations connecting the angle-sums and volume of a polytope in space of $n$ dimensions // Proc. Roy. Soc. London Ser. A. 1927. V. 115. P. 103-119.

[79] Stanley R. Hilbert functions of graded algebras // Adv. Math. 1978. V. 28. P. 57-83.

[80] Stanley R. The number of faces of simplicial convex polytope // Adv. Math. 1980. V. 35. P. $236-238$.

[81] Stanley R. A monotonicity property of $h$-vectors and $h^{*}$-vectors // European J. Combin. 1993. V. 14. P. 251-258.

[82] Stanley R. Combinatorics and Commutative Algebra. Boston: Birkhäuser, 1996. 
[83] Stanley R. Positivity problems and conjectures in algebraic combinatorics // Mathematics: Frontiers and Perspectives / ed. V. Arnold et al. Providence, RI: Amer. Math. Soc., 2000. P. 295-319.

[84] Стонг Р. Заметки по теории кобордизмов. М.: Мир, 1973.

[85] Tay T., White N., Whiteley W. Skeletal rigidity of simplicial complexes. I, II // European. J. Combin. 1995. V. 16. № 4. P. 381-403; № 5. P. 503-523.

[86] Тиморин В. А. Аналог соотношений Ходжа-Римана для простых выпуклых многогранников // УМН. 1999. Т. 54. № 2. С. 113-162.

[87] Васильев В. А. Топология дополнений к дискриминантам. М.: Фазис, 1997.

[88] Yuzvinsky S. Small rational model of subspace complement // Preprint, 1999; http:// xxx.lanl.gov/abs/math.C0/9806143.

[89] Ziegler G. Lectures on Polytopes. New York: Springer-Verlag, 1995.

Московский государственный университет им. М. В. Ломоносова

E-mail: buchstab@mech.math.msu.su, buchstab@mendeleevo.ru;

tpanov@mech.math.msu.su
Поступила в редакцию 10.08 .2000 